\title{
WestVirginiaUniversity
}

THE RESEARCH REPOSITORY @ WVU

Graduate Theses, Dissertations, and Problem Reports

2016

\section{Sphingolipid Metabolism and Insulin Resistance in Dairy Cattle}

Jorge Eduardo Rico Navarrete

Follow this and additional works at: https://researchrepository.wvu.edu/etd

\section{Recommended Citation}

Rico Navarrete, Jorge Eduardo, "Sphingolipid Metabolism and Insulin Resistance in Dairy Cattle" (2016). Graduate Theses, Dissertations, and Problem Reports. 7238.

https://researchrepository.wvu.edu/etd/7238

This Dissertation is protected by copyright and/or related rights. It has been brought to you by the The Research Repository @ WVU with permission from the rights-holder(s). You are free to use this Dissertation in any way that is permitted by the copyright and related rights legislation that applies to your use. For other uses you must obtain permission from the rights-holder(s) directly, unless additional rights are indicated by a Creative Commons license in the record and/ or on the work itself. This Dissertation has been accepted for inclusion in WVU Graduate Theses, Dissertations, and Problem Reports collection by an authorized administrator of The Research Repository @ WVU.

For more information, please contact researchrepository@mail.wvu.edu. 
Sphingolipid Metabolism and Insulin Resistance in Dairy Cattle

Jorge Eduardo Rico Navarrete. M.S.

Dissertation submitted

to the Davis College of Agriculture, Natural Resources and Design at West Virginia University

In partial fulfillment of the requirements for the degree of

Doctor of Philosophy in

Animal and Food Science

Joseph W. McFadden, Ph.D., Chair

F. Brad Hillgartner, Ph.D.

Robert A. Dailey, Ph.D.

Norman J. Haughey, Ph.D.

Janet C. Tou, Ph.D.

Division of Animal and Nutritional Sciences

Morgantown, West Virginia

2016

Keywords: Ceramide, dairy cow, insulin, sphingolipid

Copyright 2016 Jorge Eduardo Rico Navarrete 


\section{ABSTRACT \\ Sphingolipid Metabolism and Insulin Resistance in Dairy Cattle \\ Jorge Eduardo Rico Navarrete}

During their productive and reproductive life cycles, dairy cows naturally undergo physiological adaptations that facilitate modifications of nutrient flux to sustain the energy demands of gestation and lactation. The onset of lipolysis from adipose tissue is necessary to sustain tissue energy supply while cows experience sustained negative energy balance during early lactation. Excessive lipomobilization, however, predisposes dairy cows to metabolic disease during postpartum, leading to poor health, reduced productive and reproductive performance, increased rates of culling, and economic losses for the industry. In monogastrics, ceramides are implicated in the pathogenesis of insulin resistance, and are associated with saturated fatty acid availability and the onset of metabolic diseases. To date, the role of ceramides and its association with insulin action during peripartum and established lactation, have not been investigated in dairy cows. The studies presented in this dissertation evaluate the associations between fatty acid availability, ceramide synthesis, and insulin action. In the first and second experiment, we established that increased body fat accumulation results in elevated circulating NEFA, ketone bodies and liver fat accumulation, reduced plasma sphingomyelin, and increased ceramide content in plasma, liver and skeletal muscle. Ceramides were inversely associated with circulating insulin and insulin sensitivity. Time-specific effects were observed for insulin ceramide accumulation, which increased during the transition from gestation to lactation, and were independent of adiposity. Similarly, plasma sphingomyelins, a source of ceramides, decreased toward parturition and increased postpartum. Ceramide accumulation in plasma was associated with hepatic fat accumulation and hepatic ceramide availability. In the third experiment, the effects of feeding palmitate $(\mathrm{C} 16: 0 ; 4 \%$ of ration $\mathrm{DM})$ were characterized in dairy cows during mid-lactation. Palmitate supplementation resulted in increased hepatic accumulation of ceramides and modulation of genes related to ceramide synthesis. Similarly, palmitate supplementation increased circulating ceramide concentration, a response both positively associated with milk yield and negatively associated with circulating NEFA disappearance during an intravenous glucose challenge. Interestingly, palmitate supplementation delayed time-associated decrease of circulating ceramide during lactation. Collectively, these results demonstrate that availability of circulating fatty acids can impact ceramide synthesis and suggest that insulin action is associated with ceramide accumulation. Moreover, time-dependent changes occur in sphingolipid availability during the cow life cycle, indicating a possible role of ceramides in the physiological adaptations that allow preservation of energy homeostasis. These studies provide a framework for the study peripartal and lactational insulin resistance in association with sphingolipid metabolism. 


\section{ACKNOWLEDGEMENTS}

This work was supported by the USDA Sustainable Agriculture Research and Education Program (\#GNE13-067), the USDA Agriculture and Food Research Initiative (AFRI) Competitive Grant (\#WVA00902), Hatch Project WVA00661 and the Davis College of Agriculture, Natural Resources and Design. The authors thank Jurgen Godau (Global Agri-Trade) for supplying the Palmit 98 supplement. We also gratefully acknowledge Neil Sprenkle, Sina Saed Samii, Yu Zang, Rachel Cokeley, and Loretta Aromeh for their support at West Virginia University, and Jackie Lovett at Johns Hopkins University School of Medicine (Baltimore, MD), for her technical assistance. Special thanks to the staff at West Virginia University Animal Science farm and the VanGilder family at DoVan Farms for their participation. 


\section{TABLE OF CONTENTS}

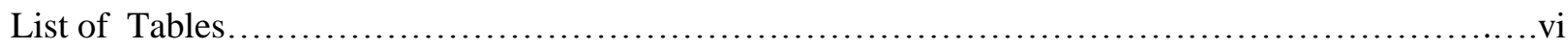

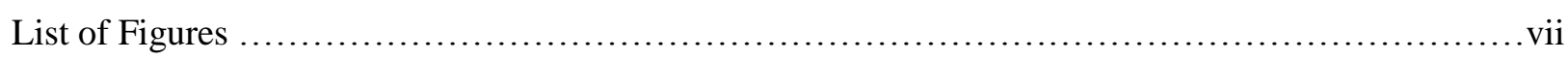

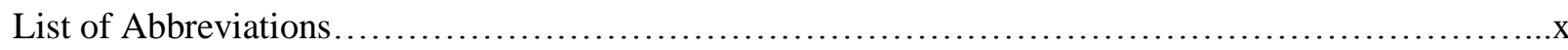

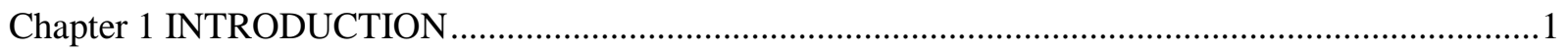

Chapter 2 LITERATURE REVIEW .............................................................................................

Energy Metabolism in the Dairy Cow ……………………...............................................

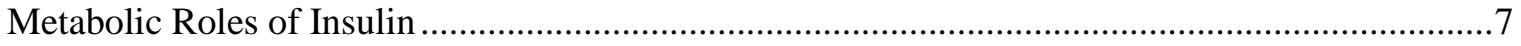

Insulin Secretion and Sensitivity in Dairy Cattle ………………………………………………10

Changes in Insulin Secretion and Action During Pregnancy and Lactation ...........................11

Mechanism of Insulin resistance ..........................................................................................13

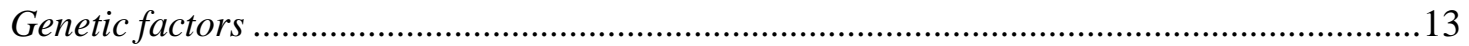

Hyperglycemia, Hyperinsulinemia, Inflammation and Cellular Stress.....................................14

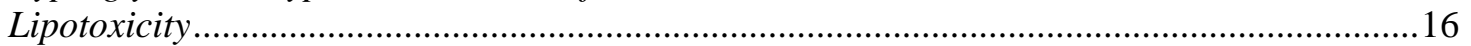

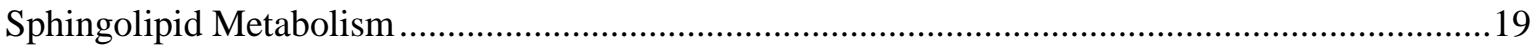

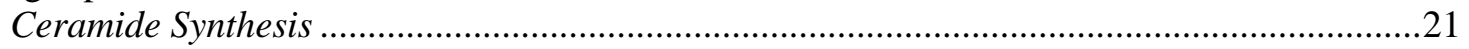

Hepatic Ceramide synthesis and Secretion ……………….............................................23

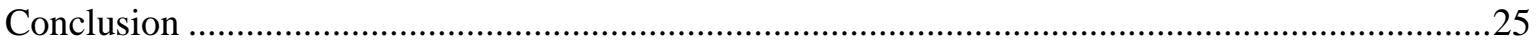

Chapter 3 PLASMA CERAMIDES ARE ELEVATED IN OVERWEIGHT HOLSTEIN DAIRY COWS EXPERIENCING GREATER LIPOLYSIS AND INSULIN RESISTANCE DURING THE TRANSITION FROM LATE PREGNANCY TO EARLY LACTATION .....................................27

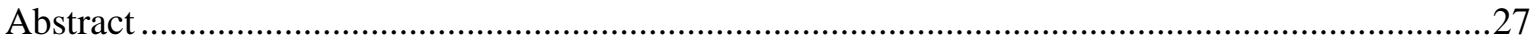

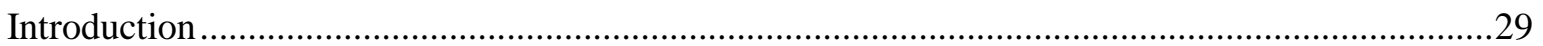

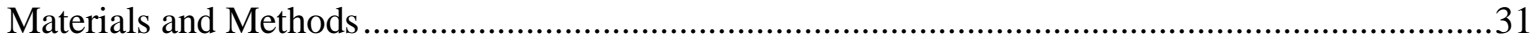

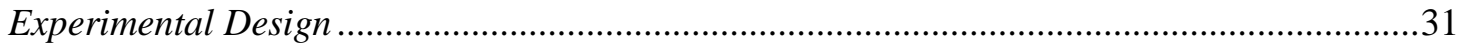

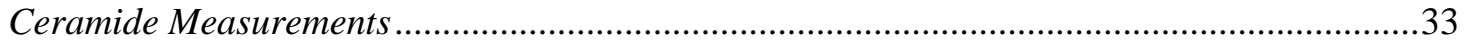

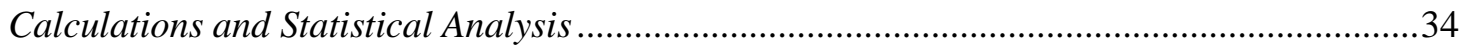

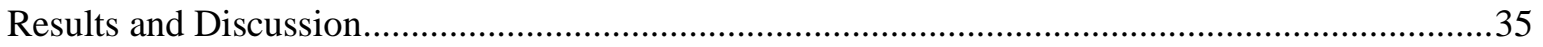

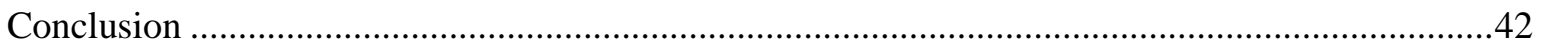

Chapter 4 TEMPORAL CHANGES IN CIRCULATING AND TISSUE SPHINGOLIPIDS DURING THE TRANSITION FROM GESTATION TO LACTATION. .......................................................58

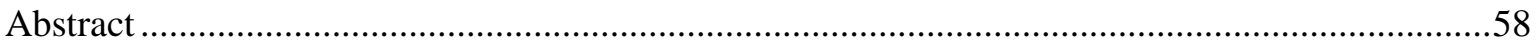

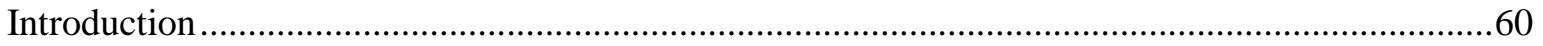

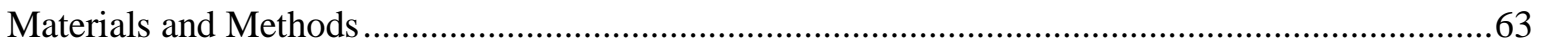

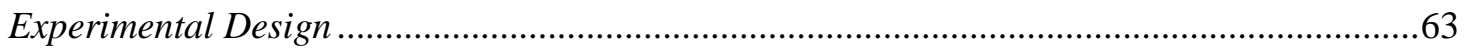

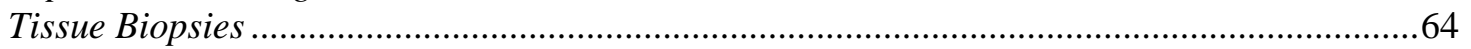

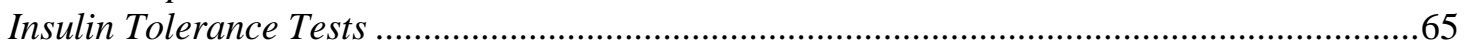

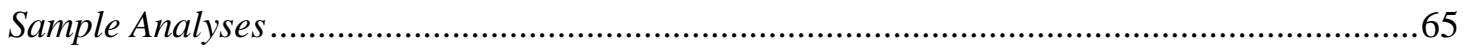

Calculations and Statistical Analysis .........................................................................67 


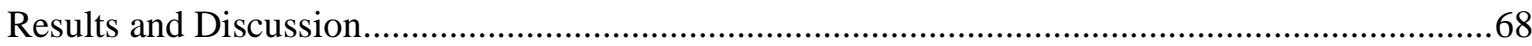

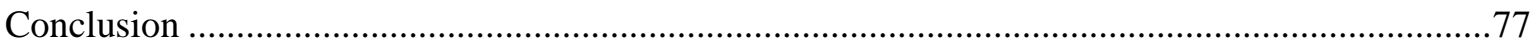

Chapter 5 PALMITIC ACID FEEDING INCREASES CERAMIDE AVAILABILITY IN ASSOCIATION WITH INCREASED MILK YIELD, NEFA AVAILABILITY, AND ADIPOSE TISSUE RESPONSIVENESS TO A GLUCOSE CHALLENGE...............................................95

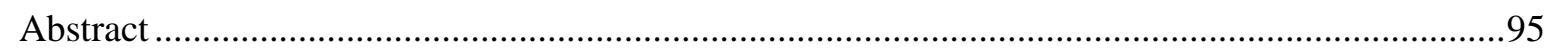

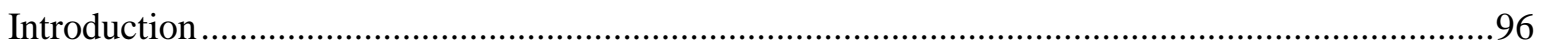

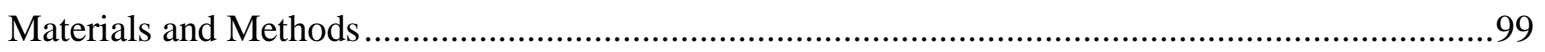

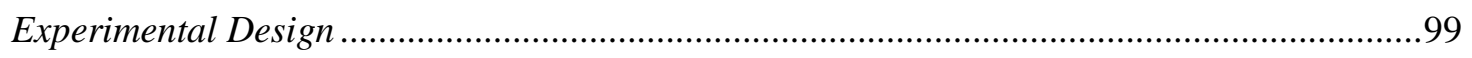

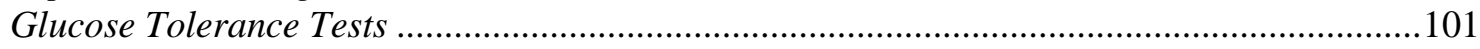

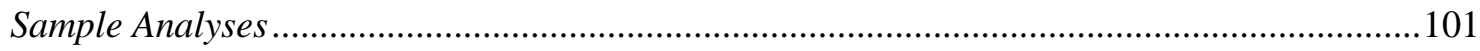

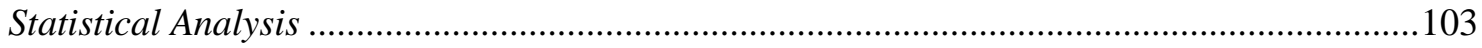

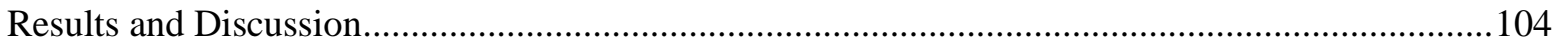

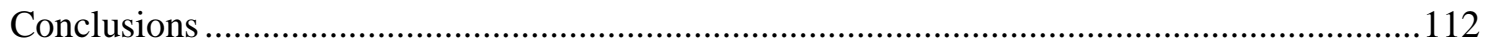

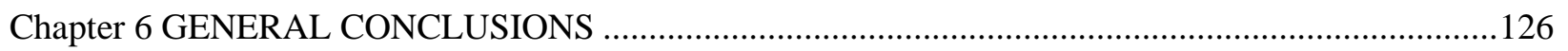

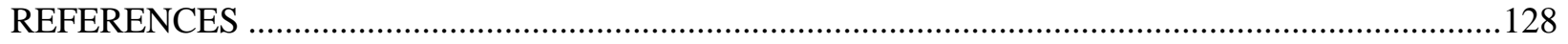




\section{LIST OF TABLES}

Table 3-1. Ingredients and nutrient composition of diets fed to lean and overweight cows during the

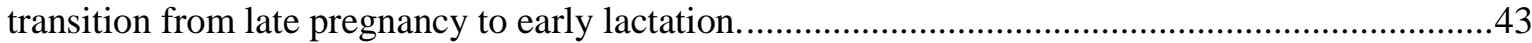

Table 4-1. Ingredients and nutrient composition of diets fed to lean and overweight cows during the transition from late pregnancy to early lactation......................................................................... 78

Table 5-1. Ingredients and nutrient composition (\% of DM unless otherwise noted) of experimental diets supplemented with palmitic acid (PALM; C16:0) and control (no added fat)...............................113

Table 5-2. Plasma ceramides are positively correlated with circulating NEFA and milk yield, and negatively associated with area under the curve of NEFA during a glucose challenge in dairy cows

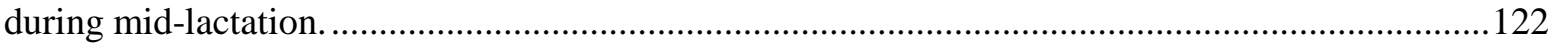

Table 5-3. Supplemental 5-1. Nucleotide and PCR product specifications .........................................125 


\section{LIST OF FIGURES}

Figure 2-1. Insulin signaling pathway 10

Figure 2-2. Changes in insulin secretion, insulin sensitivity and circulating NEFA during the cow life

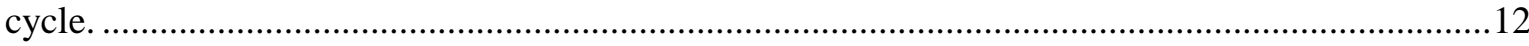

Figure 2-3. Lipotoxic theory of insulin resistance in myocytes and hepatocytes. ................................18

Figure 2-4 Ceramide acyl chain diversity and the specificity of (dihydro)ceramide synthases...............20

Figure 2-5. Ceramide synthesis and degradation. Main pathways contributing to cellular accumulation of ceramides.

Figure 2-6. Hypothesized model of ceramide synthesis and transport into insulin-producing and insulinsensing tissues.

Figure 3-1. Metabolic pathways of ceramide synthesis.

Figure 3-2. Overweight dairy cows lost more prepartum body condition and weight, relative to lean cows.

Figure 3-3. Overweight dairy cows are insulin resistant before onset of lipolysis.

Figure 3-4. C16:0- and C24:0-ceramide are high-abundant ceramides in bovine plasma.

Figure 3-5. Plasma ceramides, monohexosylceramides, and lactosylceramides are elevated in overweight dairy cows transitioning from gestation to lactation.

Figure 3-6. Plasma concentration $(\mathrm{ng} / \mathrm{mL})$ of total ceramides, monohexosylceramides, and lactosylceramides in lean and overweight cows transitioning from late pregnancy to early lactation, relative to lean cows.

Figure 3-7. Plasma concentration (ng/mL) of C24:0-linked ceramide, monohexosylceramide, and lactosylceramide is elevated in overweight cows transitioning from late pregnancy to early lactation, relative to lean cows.

Figure 3-8. Plasma C24:0-ceramide is positively associated with C24:0-monohexosylceramide, and C24:0-monohexosylceramide is positively associated with C24:0-lactosylceramide.

Figure 3-9. Plasma ceramides are positively correlated with circulating NEFA, and negatively correlated with insulin sensitivity in dairy cows transitioning from late pregnancy to lactation.

Figure 3-10. Supplemental 3-1. Plasma concentrations $(\mathrm{ng} / \mathrm{mL})$ of low-abundant ceramides in lean and overweight cows transitioning from late pregnancy to early lactation, relative to lean cows.

Figure 3-11. Supplemental 3-2. Plasma concentrations (ng/mL) of C16:0-linked dihydroceramide, dihydro-monohexosylceramide, and dihydro-lactosylceramide in lean and overweight cows transitioning from late pregnancy to early lactation, relative to lean cows. 
Figure 3-12. Supplemental 3-3. Plasma C16:0-dihydroceramide is positively correlated with C16:0ceramide.

Figure 3-13. Supplemental 3-4. Plasma concentrations (ng/mL) of low-abundant monohexosylceramides in lean and overweight cows transitioning from late pregnancy to early lactation, relative to lean cows.

Figure 3-14. Supplemental 3-5. Plasma concentrations (ng/mL) of low-abundant lactosylceramides in lean and overweight cows transitioning from late pregnancy to early lactation, relative to lean cows....57

Figure 4-1. Major pathways of ceramide synthesis.

Figure 4-2. Plasma ceramides, monohexosylceramides (GlcCer), and lactosylceramides (LacCer) are elevated in overweight dairy cows transitioning from gestation to lactation.

Figure 4-3. Plasma ceramides change dynamically during the transition from gestation to lactation.......81

Figure 4-4. Plasma ceramides are elevated in overweight cows during the transition from gestation to lactation.

Figure 4-5. Plasma concentrations (ng/mL) of monohexosylceramides (GlcCer) in lean and overweight cows transitioning from gestation to lactation.

Figure 4-6. Plasma concentrations (ng/mL) of lactosylceramides (LacCer) in lean and overweight cows transitioning from gestation to lactation

Figure 4-7. Plasma sphingomyelin profile during the transition from gestation to lactation. .85

Figure 4-8. Plasma sphingomyelins change dynamically in dairy cows transitioning from gestation to lactation.

Figure 4-9. Plasma sphingomyelins decrease toward parturition and increase postpartum .87

Figure 4-10. Ceramide profile of liver and skeletal muscle at d 4 postpartum. .88

Figure 4-11. C24:0-ceramide and total ceramide hepatic accumulation increase in overweight cows during the transition from gestation to lactation.

Figure 4-12. C24:0-ceramide and total ceramide content of skeletal muscle during the transition from gestation to lactation.

Figure 4-13. Plasma ceramide content is associated with hepatic ceramide accumulation postpartum. ..91

Figure 4-14. Circulating ceramides are inversely associated glucose disappearance during an insulin challenge at $\mathrm{d} 4$ postpartum.

Figure 4-15. Supplemental Figure 1. Plasma dihydro-sphingomyelins (DH) decrease toward parturition and increase postpartum. 
Figure 4-16. Supplemental Figure 2. Monohexosylceramide (GlcCer) and lactosylceramide (LacCer) profile of liver and skeletal muscle at $\mathrm{d} 4$ postartum.

Figure 5-1. De novo ceramide synthesis.

Figure 5-2. C24:0-ceramide is the most abundant ceramide in plasma, and liver and skeletal muscle tissue of lactating dairy cows fed control or palmitic acid-supplemented diets (PALM).

Figure 5-3. Palmitic acid-feeding (PALM) increases hepatic ceramide concentrations (ng/mg).....

Figure 5-4. Plasma ceramides and monohexosylceramides (GlcCer) are elevated in palmitic acid-fed (PALM) cows, relative to control cows.

Figure 5-5. Palmitic acid-feeding (PALM) increases total ceramide and monohexosylceramide (GlcCer) concentrations $(\mathrm{ng} / \mathrm{mL})$ in plasma.

Figure 5-6. Plasma concentration (ng/mL) of C24:0-ceramide is elevated in palmitic acid-fed (PALM) cows, relative to control cows.

Figure 5-7. Plasma concentration (ng/mL) of C24:0-monohexosylceramide (GlcCer) is elevated in palmitic acid-fed (PALM) cows, relative to control cows.

Figure 5-8. Hepatic expression of ceramides synthase (CerS) and acid sphingomyelinase (SMase) is lower in cows fed palmitic acid (PALM) for an extended duration, relative to control cows.

Figure 5-9. Supplemental 5-1. Plasma concentration (ng/mL) of lactosylceramide (LacCer) in control and

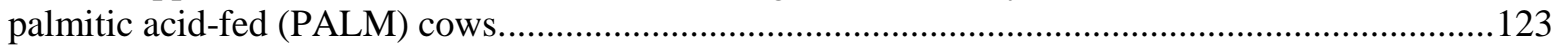

Figure 5-10. Supplemental 5-2. Plasma C24:0-monohexosylceramide (GlcCer) is positively associated with C24:0-ceramide in lactating dairy cows 


\section{LIST OF ABBREVIATIONS}

$\begin{array}{ll}\text { ADF } & \text { Acid detergent fiber } \\ \text { Akt } & \text { Protein kinase B } \\ \text { AS160 } & \text { Akt substrate } 160 \\ \text { AOAC } & \text { Association of analytical communities } \\ \text { AUC } & \text { Area under the curve } \\ \text { BCS } & \text { Body condition score } \\ \text { BHBA } & \text { Beta-hydroxybutyric acid } \\ \text { BW } & \text { Body weight } \\ \text { CE } & \text { Cholesteryl ester } \\ \text { CerS } & \text { Ceramide synthase } \\ \text { CoASH } & \text { Coenzyme A } \\ \text { CP } & \text { Crude protein } \\ \text { CPT } & \text { Carnitine palmitoyl transferase } \\ \text { CV } & \text { Coefficient of variation } \\ \text { DAG } & \text { Diacylglycerol } \\ \text { DH } & \text { Dihydro } \\ \text { DIM } & \text { Days in milk } \\ \text { DM } & \text { Dry matter } \\ \text { DMI } & \text { Dry matter intake } \\ \text { ER } & \text { Fndoplasmic reticulum } \\ \text { FAtty acid } \\ \text { FA }\end{array}$


FoxO1 Forkhead box protein 1

GLM generalized linear model

GLUT4 Glucose transporter 4

GM3 Monosialodihexosylganglioside

GSK4 Glycogen synthase kinase 3

GTT Glucose tolerance test

HDL High density lipoprotein

HPLC High performance liquid chromatography

IL-6 Interleukin 6

IRS Insulin receptor substrate

IKK I kappa B kinase beta

ITT Insulin tolerance test

JNK c-Jun N-terminal kinases

LDL Low density lipoprotein

MAPK Mitogen-activated protein kinase

mTORC1 Mammalian target of rapamycin C1

MTP Microsomal triglyceride transfer protein

NDF Neutral detergent fiber

NEB Negative energy balance

NEFA Non-esterified fatty acids

NEl Net energy for lactation

NFkB Nuclear factor kappa-light-chain-enhancer of activated B cells

OVER Overweight cow

PALM Palmitic acid treatment

PDK Phosphoinositide-dependent kinase

PH Plekstrin homology 


\begin{tabular}{|c|c|}
\hline PIP & Phosphatidylinositol \\
\hline PI3K & Phosphatidyl inositol 3 kinase \\
\hline $\mathrm{PKC}$ & Protein kinase $\mathrm{C}$ \\
\hline PL & Phospholipid \\
\hline PP2A & Protein phosphatase $2 \mathrm{~A}$ \\
\hline PTEN & Protein tensin homologue phosphatase \\
\hline ROS & Reactive oxygen species \\
\hline \multicolumn{2}{|c|}{ RQUICKI Revised Quantitative Insulin Sensitivity Check Index } \\
\hline SCS & Somatic cell score \\
\hline SEM & Standard error of the mean \\
\hline SD & Standard deviation \\
\hline SFA & Saturated fatty acids \\
\hline $\mathrm{SHC}$ & SHC transforming protein \\
\hline $\mathrm{SH} 2$ & Src-homology \\
\hline SMase & Sphingomyelinase \\
\hline SPT & Serine palmitoyl transferase \\
\hline SREBP & Sterol regulatory element binding protein \\
\hline Ss & Sphingosine \\
\hline TAG & Triglycerides \\
\hline TLR4 & Toll-like receptor 4 \\
\hline $\mathrm{TNF}-\alpha$ & Tumor necrosis factor alfa \\
\hline TMR & Total mixed ration \\
\hline Tyr & Tyrosine \\
\hline UPR & Unfolded protein response \\
\hline VLDL & Very low density lipoprotein \\
\hline
\end{tabular}




\section{Chapter 1 \\ INTRODUCTION}

The historical interest in optimizing milk production systems has led to the implementation of improved management practices, better nutrition and intense genetic selection. Over the last century, individual milk yield has increased from nearly $3000 \mathrm{~kg}$ to more than $8000 \mathrm{~kg}$ per cow per year, while greatly reducing the number of animals required to sustain the growing market demands (Capper et al., 2009). The elevated genetic merit for milk production has led to a simultaneous increase in energy requirements of cows to sustain lactation energy requirements, and has imposed more severe negative energy balance over longer periods of time during early lactation (Coppock and Wilks, 1991). In consequence, modern dairy cows experience reduced reproductive efficiency and higher propensity for metabolic stress and metabolic disease (Leroy et al., 2008; LeBlanc, 2010; Sordillo and Raphael, 2013). Although adipose tissue lipolysis is an important mechanism allowing the use fatty acids to contribute energy to peripheral tissues and to maintain glucose homestotasis, it is recognized that excessive lipomobilization predisposes dairy cows to metabolic stress and disease (Ospina et al., 2010a; Dechow et al., 2004), and it is directly related to body adiposity (Kim and Suh, 2003) and the extent of adipose tissue-specific insulin resistance during peripartum (Zachut, et al., 2013). The association between circulating lipids, body fat accumulation, and insulin resistance is well established in monogastrics and ruminants (Boden et al., 1991; Oikawa and Oetzel, 2006; Pires et al., 2007a), and it constitutes a major factor underlying the pathogenesis of the metabolic syndrome in human obesity (Varman et al., 2010). In particular, saturated fatty acids (SFA) can impair insulin action by causing decreased pancreatic insulin secretion and by inducing peripheral insulin resistance. These effects are largely driven by the accumulation of ceramides, sphingolipids that accumulate during obesity and disrupt cellular 
insulin signaling and insulin secretion (Sjoholm, 1995; Kleppe, et al., 2003; Bikman and Summers, 2011). Therefore, we aimed to study the associations between sphingolipid metabolism, fatty acid availability, and insulin action in overweight dairy cows. In addition, because lipid availability can be manipulated through the diet, we explored the effects of fat supplementation on ceramide metabolism as a mean to sustain the physiological insulin resistance that accompanies lactation and facilitates milk yield. In order to achieve our objectives, we employed a mass spectrometrybased lipidomics approach, which has been extensively utilized to characterize the roles of sphingolipids in metabolism, and their involvement in the pathogenesis of insulin resistance (Merril et al., 2005; Hannun and Obeid, 2008). We thus aimed to identify the most relevant ceramide forms associated with fatty acid availability and insulin action during the critical peripartal period and during established lactation in dairy cows. 


\section{Chapter 2}

\section{LITERATURE REVIEW}

\section{Energy Metabolism in the Dairy Cow}

From a digestive physiology perspective, dairy cows can be as defined pre-gastric fermentation herbivores. The development of the rumen as a pregastric fermentation chamber, which houses a complex microbial ecosystem, allows cows to utilize fiber and fiber-associated nutrients. This remarkable symbiotic relationship provides the dairy cow with otherwise unavailable metabolizable energy sources, such as the volatile fatty acids acetate, propionate and butyrate, as well as with high quality protein of microbial origin.

In dairy cows, most of circulating glucose is utilized by the skeletal muscles, the gravid uterus, and the udder, with the latter accounting for 50-85\% of whole-body glucose needs during early lactation (De Koster and Opsomer, 2013). Because of the extensive fermentation of dietary glucose by ruminal microorganisms, intestinal absorption of glucose is negligible (Mills et al., 1999), particularly when compared with non-ruminants (Aschenbach et al., 2010). In consequence, circulating glucose is typically low (Annison and White, 1961, Zachut et al 2013; Hammon et al., 2009), and cows rely heavily on endogenous glucose production through gluconeogenesis (Aschenbach et al., 2010), a process that predominantly utilizes circulating propionate (60-74\% of total de novo glucose). Endogenous glucose production is paramount because it allows glucose supply to reach organs that rely solely on glucose as an energy source, such as red blood cells, brain cells, and kidney cells (Aschenbach et al., 2010). Importantly, hepatic glucose production accounts for the vast majority of circulating glucose required during late gestation and early 
lactation, a period in which glucose requirements can increase 3- to 4-fold in modern dairy cows (Bell, 1995; Reynolds et al., 2003).

The transition from gestation to lactation imposes a great metabolic challenge for modern dairy cows, whose energy requirements can typically double as lactation is initiated (Drackley et al., 2005). Because dry matter, and therefore, nutrient intake, is decreased around parturition, transition dairy cows experiencing increased energy demands will commonly undergo negative energy balance (NEB) during this period (Grummer et al., 2004). This condition results in an increased use of energy from body tissues, such as adipose (lipolysis) and skeletal muscle (amino acid breakdown), as well as increased hepatic gluconeogenesis (Graber et al., 2010), all of which favor the production of glucose for lactose synthesis in the mammary gland (Bell, 1995). One of the hallmarks of NEB postpartum is the elevation in the concentration of circulating non-esterified fatty acids (NEFA), mobilized from adipose tissue triacylglycerols (TAG). Adipose tissue metabolism shifts from lipogenic to lipolytic during this period (McNamara, 1991), and this process is facilitated by changes in the levels of plasma homeorhetic hormones (Tucker, 1985), a decrease in insulin concentration, and a progressively increased refractoriness of peripheral tissues to the action of insulin (Bell and Bauman, 1997). In addition, adipose tissue sensitivity to lipolytic hormones such as epinephrine and norepinephrine is greatly enhanced, while circulating glucagon concentrations increase (Drackley et al., 2005; De Koster and Opsomer, 2013; Mann et al., 2016)

The central role of the liver in energy metabolism is well exemplified during the transition from gestation to lactation. In addition to being a major gluconeogenic organ, the liver can also take up and metabolize circulating NEFA from adipose tissue TAG mobilization. Once NEFA enter the liver, fatty acids can be oxidized, serve as a carbon source for ketogenesis, or be reesterified into TAG (Drackley et al., 2005). Because ruminants have an inherently low capacity 
to export TAG to circulation through very low density lipoprotein secretion (VLDL; Kleppe et al., 1988; Pullen et al., 1989), excessive NEFA uptake can result in TAG accumulation and fatty liver may develop (Bobe et al., 2004). In addition, excessive NEFA uptake from NEB can result in increased synthesis of ketone bodies (e.g. BHBA) leading to ketosis and associated health disorders, such as mastitis, metritis, milk fever, and displaced abomasum (Herdt, 2000; Bobe et al., 2004).

The incidence of disorders of energy metabolism is greatest during the transition from late gestation to early lactation. Although the dairy cow is physiologically well suited to respond to the elevated glucose demands of the mammary gland during early lactation, intense genetic selection for elevated milk yield has led to ever more dramatic changes in metabolic activity associated with severe NEB. Pronounced metabolic adaptations to NEB, such as excessive lipolysis, are associated with dysfunctional immune responses, metabolic stress, and metabolic disease (Sordillo et al., 2009; Contreras et al., 2010; Sordillo et al., 2013). In this context, it is argued that the ability of the dairy cow to compensate for the gap between nutrient demand and energy supply has been historically overstressed, bringing about increased incidence of metabolic disorders (Sundrum, 2015).

The use of metabolic markers during prepartum has the potential to aid in the identification of early manifestations aberrant metabolism that will ultimately result in peripartal health disorders, and thereby guide herd management modifications to reduce the incidence of disease. Currently used indicators of metabolic stress, as well as subclinical and clinical metabolic disease, include the stress and inflammation marker haptoglobin, and the NEB markers NEFA and BHBA (Huzzey et al., 2015; Ospina et al., 2010a, Ospina et al., 2010b). However, the identification of pre-onset markers of metabolic abnormalities associated with increased susceptibility of cows to 
suffer from peripartal health complications is still needed. As mentioned previously, excessive lipolysis drives elevated circulating NEFA and can result in fatty liver and associated complications postpartum. Recent research shows that adipose tissue insulin resistance is associated with increased lipolysis in cows prone to lose excess body weight postpartum (Zachut et al., 2013). Overweight cows are more susceptible to lose body weight and adiposity during peripatum (Kim and Suh, 2003; Roche et al., 2009), and are at a higher risk of developing postpartum health disorders and experiencing reduced productive and reproductive performance postpartum (Fronk et al., 1980; Kim and Suh, 2003; Roche et al., 2009). The association between fat mass accumulation and metabolic disease in dairy cows has been recognized for decades and denominated 'fat cow syndrome' (Morrow, 1975). Interestingly, the symptomatology of this syndrome resembles that usually observed in overweight and obese humans diagnosed with metabolic syndrome (Boucher et al., 2104). Common features include abnormal levels of blood lipids, hepatic fat accumulation and reduced insulin action (Ohtsuka et al., 2001; O'Boyle et al., 2006; Boucher et al., 2014). Given the major role of insulin in the pathogenesis of metabolic disease it has been suggested that the term 'insulin resistance syndrome' should be used to better describe insulin's major role in the onset of disease (Reaven, 1988). Because insulin is a central regulator of energy metabolism during this critical period, understanding of factors affecting insulin secretion and action is a necessary step to better understand the etiology of peripartal disease in dairy cows. 


\section{Metabolic Roles of Insulin}

Insulin is an anabolic peptide hormone composed of 110 aminoacid residues, and it is synthesized in the $\beta$-cells of the pancreatic islets of Langerhans in response to circulating segregatogues. Its primary structure was first determined by Frederick Sanger in 1951, and it consists of two polypeptide chains, called A- and B- chains, linked together by two disulfide bonds (Sanger and Tuppy, 1951). Insulin is encoded as preproinsulin by the INS gene, and its structure has remained highly conserved across vertebrates for over 400 million years (Steiner et al., 1985; Ebberink et al., 1989; Zhang and Steiner., 2000). In fact, bovine insulin differs from human insulin in three aminoacid residues, while porcine insulin does so in only one. This strong homology is suggestive of a fundamental role of insulin as a vital metabolic signal throughout animal evolutionary history.

Insulin signals several body tissues, from the brain to liver, skeletal muscle, and adipose tissue. Globally, insulin acts as an anabolic hormone, promoting energy storage and suppressing catabolism in insulin-sensitive tissues. Although the effect of insulin on glycemic control is its most commonly discussed function, insulin governs a multitude of cellular processes to maintain energy homeostasis. The major effects of insulin on liver, adipose tissue and skeletal muscle include: (a) increased rate of glucose transport across the cellular plasma membrane, (b) increased rates of glycolysis, (c) stimulation of glycogen synthesis, suppression of glycogen breakdown and hepatic gluconeogenesis, (d) reduction in rates of adipose tissue lipolysis, (e) stimulation of de novo lipogenesis, TAG synthesis, and circulating NEFA uptake in adipose tissue and muscle, (f) reduction of fatty acid oxidation in muscle and liver, $(\mathrm{g})$ increased rates of amino acid transport into tissues and protein synthesis, and (h) reduction of protein degradation rates in muscle (Edgerton et al., 2006; Dimitriadis et al., 2011). In order to exert its anabolic actions, insulin is 
released into circulation and sensed by the insulin receptor, which relays the insulin signal into the cells by promoting phosphorylation and activation of downstream target proteins (Figure 2-1).

The insulin receptor is a transmembrane protein that belongs to the broader family of receptor tyrosine kinases (Ullrich et al., 1985; Lemmon and Schlessinger, 2010). The receptor is a heterotetrameric glycoprotein consisting of two $\alpha$-subunits and two $\beta$-subunits linked by disulfide bonds. The $\alpha$-subunits are extracellular and contain the insulin binding site, whereas the $\beta$-subunits span the plasma membrane and are involved in intracellular signaling (Hedo and Simpson, 1984). The insulin receptor is widely distributed on mammalian tissues, and its concentration varies from as few as 40 receptors on red blood cells to more than 200,000 receptors on adipocytes and hepatocytes (Khan and White, 1988). Insulin action in target tissues is initiated by a highly specific binding to the insulin receptor (Freychet et al., 1971; Czech, 1984; Menting et al., 2013). Interestingly, the binding properties of the insulin receptor in vertebrates are better evolutionarily conserved than are the functional properties of insulin itself (Muggeo et al., 1979).

When insulin binds to the $\alpha$-subunit of the insulin receptor, the intracellular $\beta$-subunit becomes activated by phosphorylation of certain tyrosine residues, a process that is commonly described as autophosphorylation, and which results in kinase activity activation of the $\beta$-subunits and further recruitment of receptor substrates (Kasuga et al., 1982; Ward and Lawrence, 2009; Figure 2-1). The insulin receptor substrates (IRS-1 through IRS-6) are a family of proteins with no intrinsic enzyme activity and act as scaffolds to mediate signaling complexes (Sun et al. 1991; White, 2006; Shaw, 2011). The activated receptor recruit IRS proteins to the cellular membrane and subsequently phosphorylate them on multiple tyrosine residues, forming binding sites for intracellular molecules that contain Src-homology 2 (SH2) domains (Sun et al., 1993). Upon IRS phosphorylation, phosphatidyl inositol 3-kinase (PI3K), a heterodimer with catalytic and 
regulatory subunits, is recruited and activated. The activation of PI3K depends on the binding of the two SH2 domains in the regulatory subunits (predominantly p85a) to tyrosine-phosphorylated IRS proteins (Myers et al. 1992; Shaw, 2011). This results in activation of the catalytic subunit (p110), which rapidly phosphorylates phosphatidylinositol 4,5-bisphosphate (PIP2) to generate the lipid second messenger phosphatidylinositol $(3,4,5)$-triphosphate (PIP3). The presence of these residues attracts molecules that express pleckstrin homology $(\mathrm{PH})$ domains, such as protein kinase B (Akt), which translocates to the inner leaflet of the plasma membrane and then binds with high affinity to 3-phosphoinositides. This process induces a conformational change that makes Akt susceptible to phosphorylation. Membrane-bound PIP3 recruits and activates the phosphoinositide-dependent kinase-1 (PDK-1), which phosphorylates Akt on threonine 308, in addition to PDK-2, which phosphorylates on serine 473, rendering Akt fully active (Saltiel and Kahn, 2001; Petersen and Shulman, 2006). Active Akt promotes the effects of insulin on several downstream proteins including the mammalian target of rapamycin-C1 (mTORC1), sterol regulatory binding-element protein-1c (SREBP1c), glycogen synthase kinase-3 (GSK-3), Akt substrate 160 (AS160), and the forkhead box protein 01 (Fox01). The major results of Akt activation include activation of lipogenesis, glycogen synthesis, glucose transport via the glucose transporter 4 (GLUT4), and the inhibition of gluconeogenesis and glycogenolysis (Figure 2-1). 


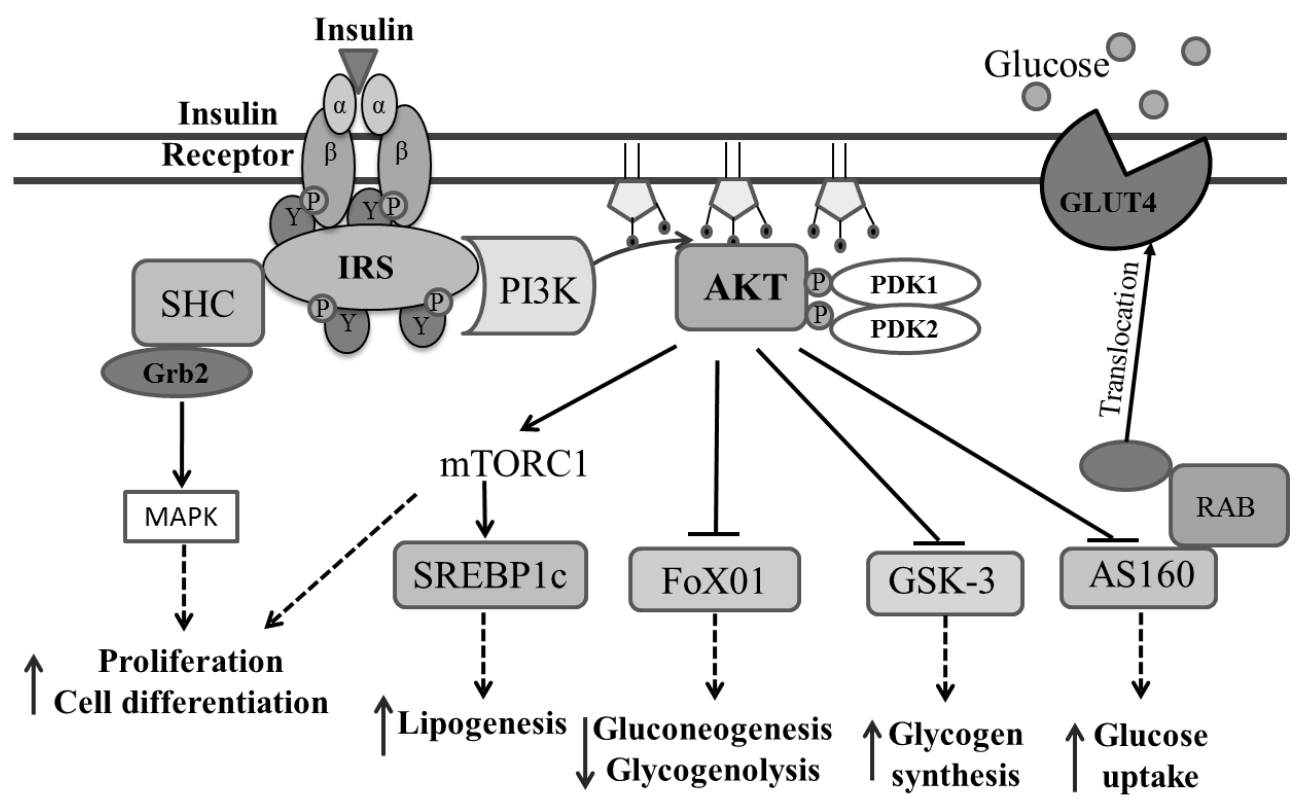

Figure 2-1. Insulin signaling pathway.

Activation of the insulin receptor by its ligands initiates a cascade of phosphorylation events which promote the metabolic effects of insulin. Abbreviations: Insulin receptor substrate (IRS), SHCtransforming protein (SHC), mitogen-activated protein kinase (MAPK), Protein kinase B (Akt), phosphoinositide-dependent kinase-1 (PDK1), phosphoinositide-dependent kinase-2 (PDK-2), mammalian target of rapamycin-C1 (mTORC1), sterol regulatory binding-element protein-1c (SREBP1c), glycogen synthase kinase-3 (GSK-3), Akt substrate 160 (AS160), forkhead box protein O1 (FoXO1), glucose transporter 4 (GLUT4). Adapted from Turner, 2013.

\section{Insulin Secretion and Sensitivity in Dairy Cattle}

In association with a unique digestive physiology, ruminants have co-evolved metabolic adaptations which allow them to maintain energy homeostasis under conditions of chronicallylimited exogenous glucose supply. The regulatory role of insulin on metabolism, for example, is preserved by the activation of alternative metabolic signals that can promote its release. Propionate, a major gluconeogenic substrate derived from ruminal fermentation of carbohydrates, is a potent insulin segregatogue in ruminants, and thus constitutes an important energy-sensing signal (Hertelendy et al., 1968; Sano et al., 1999; Bradford et al., 2006). The importance of 
circulating propionate on insulin secretion is underscored by the observation that pancreatic responsiveness to propionate is higher than that to glucose itself (Manns and Boda, 1967).

The glucoregulatory response of peripheral tissues to insulin signals is also comparatively low in ruminants, relative to non-ruminants (Annison and White, 1961; Kaske et al., 2001, Sasaki, 2002). The fact that ruminants maintain a homeostatic status in spite of reduced insulin action seems paradoxical. This resistance to the stimulatory action of insulin on glucose metabolism may be due to the lower content of GLUT4 and the lower capacity of insulin signal transduction, which results in lower glucose transport activity (Sasaki, 2002), and may be reflective of a constantly reduced glucose supply to insulin-sensitive tissues.

\section{Changes in Insulin Secretion and Action During Pregnancy and Lactation}

As mentioned previously, major shifts in nutrient partitioning are required to support fetal growth and milk synthesis during the peripartal period (Bauman and Currie, 1980; Bell, 1995). These metabolic shifts encompass changes in circulating endocrine regulators, such as growth hormone, estrogens, progesterone, and insulin, among others (Tucker, 1985). The process is known as homeorhesis, and it was first proposed by Waddinton in 1957 as 'flow toward a new equilibrium', in a clear analogy to the equilibrium of homeostasis. Bauman and Currie crystalized the concept as 'the orchestrated changes in metabolism of body tissues necessary to support a physiological state' (Bauman and Currie, 1980). Insulin plays a major role in the regulation of macronutrient tissue metabolism, and it is fundamentally involved in homeostasis and homeorhesis during peripartum (Bauman Currie, 1980). Both insulin concentration and insulin responsiveness change dynamically during the transition from gestation to lactation (Bauman and Currie, 19080; 
Bell, 1995; Bell and Bauman, 1997; Figure 2-2). Indeed, insulin secretion is elevated during the majority of pregnancy and decreases as parturition approaches in several animal species, including cows and humans (Bell and Bauman, 1997; Homko et al., 2001; Zachut et al., 2013). Similarly, it is well recognized that insulin resistance develops during late pregnancy in monogastrics (Ryan et al., 1985; Sevillano et al., 2007), while in the dairy cow, postpartum insulin sensitivity is effectively lower than during prepartum (Bell and Bauman, 1997; Kerestes et al., 2009). Importantly for dairy cows, reduced insulin action results in increased hepatic gluconeogenesis and increased adipose tissue lipolysis, both of which favor the preservation of energy homeostasis during the unavoidable progression of postpartum NEB (Figure 2-2). Recent evidence demonstrates that localized insulin resistance occurs in the adipose tissue of early lactation dairy cows, mechanistically driven by decreased tyrosine phosphorylation of IRS-1 (Ji et al., 2012; Zachut et al., 2013).

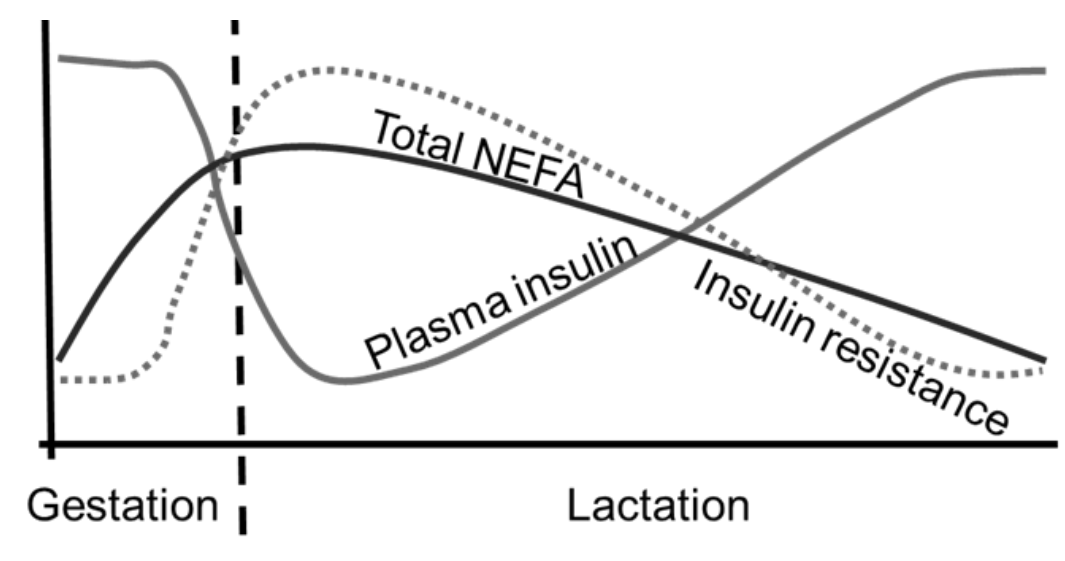

Parturition

Figure 2-2. Changes in insulin secretion, insulin sensitivity and circulating NEFA during the cow life cycle.

Abbreviations: Non-esterified fatty acids (NEFA). 


\section{Mechanism of Insulin resistance}

The major mechanisms driving insulin resistance will be discussed briefly. For an extended reading, the reviews by Hotamisligil, 2006, Varman et al., 2010, De Koster and Opsomer (2013), and Boucher et al. (2014), are recommended.

\section{Genetic Factors}

Point mutations in the insulin receptor and insulin signaling molecules such as IRS, PI3K, PDK2 and AS160 result in increased protein turnover, reduced expression and ligand affinity, and decreased insulin signaling capacities, all described in severe genetic forms of insulin resistance (Boucher et al., 2014). Similarly, single nucleotide polymorphisms of the protein phosphatase tensing homolog (PTEN) lead to reduced Akt activation and associated downstream proteins via reduced PIP3 levels (Ishihara et al., 2003). Interestingly, genetic haploinsufficiency of PTEN results in reduced dephosphorylation of PI3K and enhanced insulin sensitivity in obese individuals. Although these subjects do not develop type 2 diabetes, they display a higher propensity to develop cancer (Pal et al., 2012). Similar to humans, genetic influences may modulate insulin sensitivity in dairy cattle. For example, cows with high genetic merit for milk production are known to be more insulin resistant that cows with a low genetic merit for milk production (Chagas et al., 2009). However, little is known of the specific genes responsible for these differences in insulin sensitivity. Further investigation is needed to characterize gene variants responsible for increased insulin resistance, as well as their role in the onset of metabolic disease. 


\section{Hyperglycemia, Hyperinsulinemia, Inflammation and Cellular Stress}

Supraphysiologycal concentrations of circulating glucose result in decreased insulin action in adipose tissue and skeletal muscle, both in vivo and in vitro (Leahy et al., 1986; Hager et al., 19991; Robinson and Buse, 2008). Because hyperglycemia is a hallmark of peripheral insulin resistance, it is conceivable that the negative effects of glucose on insulin signaling (i.e. glucotoxicity) are secondary to other initial causes. This implies that glucose is part of a negative cycle that exacerbates initial insulin resistance. Interestingly, inhibition of glucose transport by inactivation of GLUT4 in rats can cause secondary defects in insulin action in adipose tissue and liver (Kim et al., 2001). Mechanistically, hyperglycemia results in glycation and O-GlcNAcylation of insulin signaling proteins, reduced receptor affinity, increased Ser-307 phosphorylation IRS-1, decreased binding of transcription factors, and increased protein phosphatase 2-A activity (Boucher et al., 2014). In addition, hyperglycemia can increase the activity of cellular phosphatases, such as PP2A and PTEN, causing reduced phosphorylation of Akt (Robinson and Buse, 2008; Kowluru and Matti, 2012).

Insulin can have dampening effects on its own pathway. This process is known as homologous desensitization and posits that continuous exposure of the target cells reduces the ability of insulin to stimulate signal transduction at the level of the receptor (Shanik et al., 2008). This negative feedback loop involves reduced receptor affinity, lower number of receptors on the

cell surface, and decreased ability of the receptor to transmit stimulatory signals. These processes encompass negative cooperativity of insulin binding (De Meyts et al., 1973; De Meyts et al., 1976), increased internalization and degradation of hormone occupied receptors (Gavin et al., 1974), reduced receptor kinase activity, and inactivation of downstream kinases via Ser and Thr phosphorylation events (Zick et al., 1983; Paz et al., 1997; Boucher et al, 2014). 
Chronic low-grade inflammation is a prominent characteristic of obesity, and it is considered to be a major component in promoting obesity-associated insulin resistance (Hotamisligil, 2006; Osborn and Olefsky, 2012). Inflammation is characterized by abnormal cytokine production (e.g. TNF- $\alpha$ ), acute phase reactants (e.g. haptoglobin), and activation of a network of inflammatory signaling pathways (Wellen and Hotamisligil, 2005; Sordillo and Raphael, 2013, De Koster and Opsomer, 2013). Adipocytes and macrophages are central cell types in the pathogenesis of insulin resistance in obesity. Typically, macrophages are recruited and infiltrate the adipose tissue promoting a state of inflammation, leading to secretion of proinflammatory cytokines and inducing insulin resistance (Schenk et al., 2008). In dairy cows, the peripartal period is associated with a high degree of inflammation (Bertoni et al., 2008) which can be exacerbated in overweight individuals and those diagnosed with fatty liver, leading to elevated NEFA, increased TNF- $\alpha$ activity, insulin resistance and metabolic disorders (O'Boyle $e t$ al., 2006; Ohtsuka et al., 2001).

Cellular stress is a well characterized cause of insulin resistance, and it is manifested by mitochondrial oxidative stress and endoplasmic reticulum (ER) stress (Evans et al., 2005; Jheng et al., 2012; Ozcan et al., 2004). Unresolved reactive oxygen species (ROS) accumulation causes oxidative stress in the mitochondrion, and results in alterations of mitochondrial proteins and reduced expression and activity of antioxidant enzymes (i.e. mitochondrial dysfunction; Evans et al. 2005; Fridlyand and Philipson 2006). Oxidative stress causes activation of stress kinases that induce serine phosphorylation and insulin resistance at the level of IRS proteins (Rudich et al. 1998; Evans et al. 2005; Dokken et al. 2008). In the liver, specifically, oxidative stress reduces FA oxidation and can lead to diacylglycerol (DAG) accrual, PKC-1 activation and decreased IRS-2 phosphorylation and PI3-kinase activity (Koh et al.2005; Zhang et al. 2007). The unfolded protein 
response (UPR) of the ER, is cellular process that ensures proper protein folding, maturation and quality control, and it is activated to reduce the unfolded protein load (Hotamisligil, 2010). Obese mice display increased activation of UPR pathways, which leads to inflammatory pathway activation (e.g. JNK and IKK) and insulin resistance via serine 307 phosphorylation of IRS-1 (Ozcan et al. 2004; Zhang et al. 2008).

Of note, the aforementioned mechanisms causing insulin resistance all converge in the activation of serine/threonine kinases, which leads to inhibitory phosphorylation of the insulin receptor, IRS proteins, and Akt, and thereby results in disruption of insulin signaling (Boucher et al., 2014).

\section{Lipotoxicity}

An important feature of the metabolic syndrome in monogastrics is the ectopic accumulation of lipids, particularly of fatty acids, as a causal factor of insulin resistance commonly associated with obesity (DeFronzo, 2004; Boucher et al., 2014). In dairy cows, the association between fat accumulation and insulin resistance was first demonstrated by MCann and Reimers (1985), who reported lower reduction of glucose following and intravenous challenge in obese heifers. Tissue accumulation of lipids constitutes a direct link for the known association between elevated circulating fatty acids and insulin resistance (Boden et al., 1991; Roden et al., 1996; Schenk et al., 2008; Figure 2-3). The effects of fatty acids, in particular, are facilitated by ectopic fat accumulation, which, besides causing insulin resistance, can result in increased lipolysis, $\beta$-cell dysfunction and perturbations of fatty acid oxidation, a cluster of abnormalities denominated lipotoxicity (Unger, 2003; DeFronzo, 2004; Wellen and Hotamisligil, 2005). Manifestations of 
lipotoxicity in non-adipose tissues are due to increased lipid transport into tissues and result in skeletal muscle insulin resistance (Ferreira et al. 2001), lipotoxic cardiomyopathy and fatty liver disease (Chiu et al. 2005; Koonen et al. 2007). In dairy cows, elevated NEFA is associated with the development of insulin resistance (Kerestes et al., 2009). Interestingly, both the use of hypercaloric diets and intravenous lipid infusion,induce elevation of plasma NEFA and result in insulin resistance (Holtenius et al., 2003; Pires et al., 2007a). Ultimately, increased NEFA results in serine phosphorylation of IRS-1, decreased downstream phosphorylation events and disruption of insulin signal transduction (Le Marchand-Brustel, et al., 2003). The effects of circulating NEFA on insulin sensitivity in different tissues are mediated by lipid intermediates formed upon cellular activation (i.e. fatty acyl CoAs), and include ceramides, DAG, and, probably, acylcarnitines (Holland and Summers, 2008; Erion and Shulman, 2010; Mihalik et al., 2010). When the flux of fatty acids (e.g. accelerated lipolysis in adipose tissue) outpaces the ability of the pathways to dispose of fatty acyl-CoAs, these intermediaries of fatty acid metabolism can accumulate (Schenk et al., 2008; Figure 2-3). 


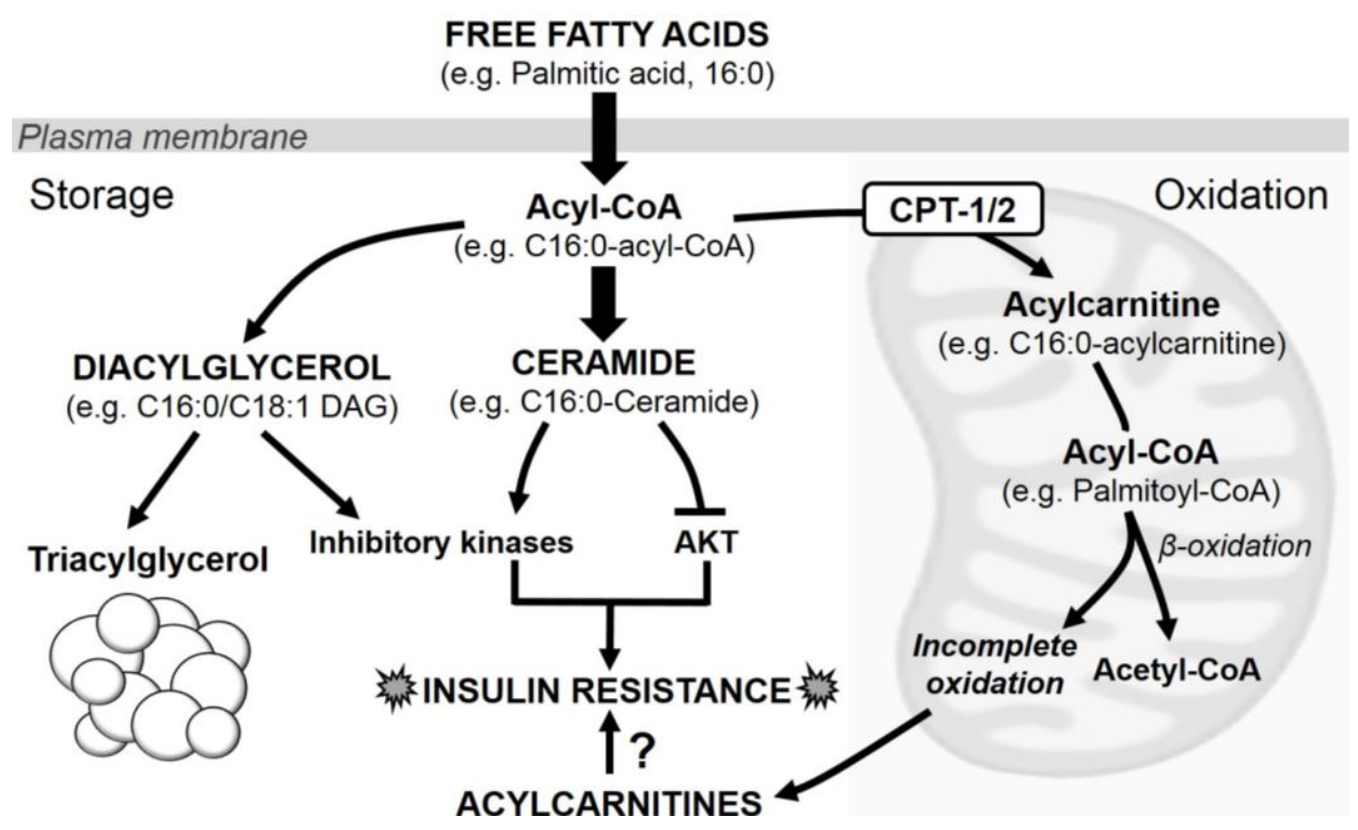

Figure 2-3. Lipotoxic theory of insulin resistance in myocytes and hepatocytes.

Abbreviations: Carnitine palmitoyl acyl transferase-1 and -2 (CPT1/2), Protein kinase B (Akt). Adapted from Schenk et al., 2008.

The effects of these lipid intermediates converge at the activation of different serine kinases, resulting in disrupted insulin signaling. Ceramides, in particular, have gained attention as effectors of insulin resistance because they are associated with obesity, and because, unlike DAG, they specifically link circulating SFA availability and insulin resistance (Chavez et al., 2003). Ceramides accumulate in insulin resistant tissues of rodents (Summers, 2006; Holland and Summers 2008) and humans (Adams et al., 2004; Straczkowski, 2007), which results in reduced Akt phosphorylation through its targets PP2A and protein Kinase C $\zeta$ (Chavez et al., 2003; Stratford et al., 2004; Bourbon et al., 2002). Saturated FA are proinflammatory compounds that lead to activation of inflammatory signaling in macrophages, adipocytes, myocytes and hepatocytes (Hwang and Rhee, 1999; Yu et al., 2002; Nguyen et al., 2005; Nguyen et al., 2007). This leads to stimulation of inflammatory proteins IKK $\beta / \mathrm{NF} K \mathrm{~B}$ and JNK/AP1 and cytokines, such as tumor 
necrosis factor- $\alpha$ (TNF- $\alpha$ ), via stimulation of the Toll-like receptor 4 (TLR4; Nguyen et al., 2007; Shi et al., 2006). Of note, the mechanisms linking SFA and inflammation seem to contribute to macrophage induced insulin resistance by converging in elevated ceramide synthesis. In dairy cows, the adipose tissue is a primary site for inflammation, and it has the ability to produce the inflammatory cytokines TNF- $\alpha$ and interleukin 6 (IL-6; Sadri et al., 2010). Although the effects of most adipokines on insulin sensitivity in dairy cattle have not been extensively characterized, the effects of TNF- $\alpha$ have been widely studied. Subcutaneous injection of TNF- $\alpha$ resulted in decreased insulin sensitivity and increased hepatic TAG accumulation in dairy cows in steers and dairy cows, respectively (Kushibiki et al 2001; Bradford et al., 2009). In addition, cows suffering from fatty liver had increased serum NEFA and plasma TNF- $\alpha$ concentrations, and were more insulin resistant relative to cows with normal fat accumulation (Ohtsuka et al., 2001). Moreover, similar to obese humans, overweight dairy cows display elevated plasma TNF- $\alpha$ concentration (O'boyle et al., 2006), providing a potential mechanistic link between fat accumulation and insulin resistance in dairy cattle.

\section{Sphingolipid Metabolism}

Sphingolipids are a class of bioactive lipids comprised of a sphingoid base backbone, sphinganine (an eighteen-carbon alcohol backbone; Hannun and Obeid, 2008; Figure 2-4). Variations of this basic structure give origin to a vast and complex family of sphingolipids (e.g. ceramides, sphingosine-1-phosphate, sphingomyelin, and gangliosides; Merrill et al., 2007), with structural and regulatory roles, including cell growth, death, senescence, adhesion, migration, 
inflammation, angiogenesis and intracellular trafficking (Hannun and Obeid 2008). Ceramides are formed by the $\mathrm{N}$-acylation of sphingosine with fatty acids (Gault et al., 2010), in a process carried out by the action of six different ceramide synthases (Levy and Futerman; Figure 2-4). Because ceramide can contain differing acyl CoAs, it is considered to be a class of molecules, rather than a single molecule, which can have different biological functions depending on the acyl chain they contain. To date, the specific bioactive roles of ceramides with differing acyl chain FA composition have not been completely elucidated.

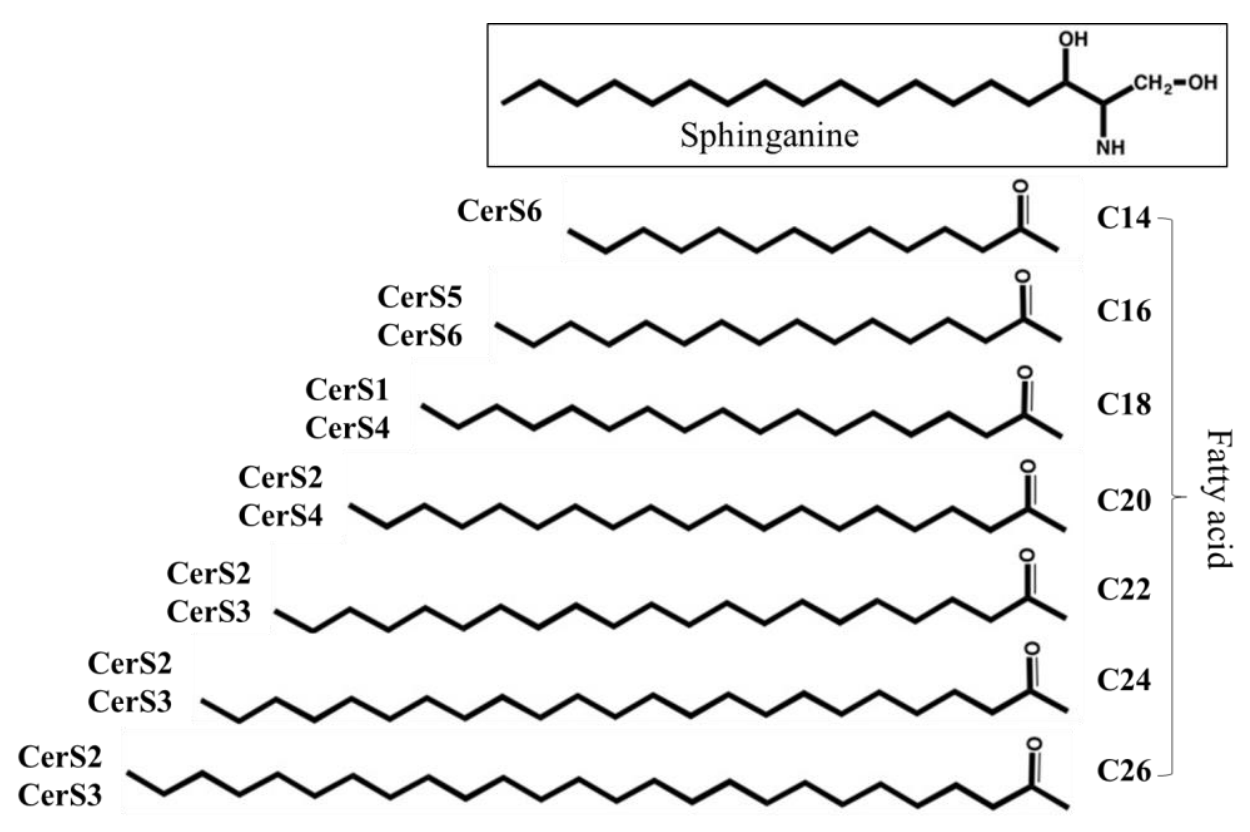

Figure 2-4 Ceramide acyl chain diversity and the specificity of (dihydro)ceramide synthases.

The N-acylation of sphinganine by (dihydro)ceramide synthases results in ceramides of different acyl chain length. CerS: Ceramide synthase. Adapted from Levy and Futerman, 2010. 


\section{Ceramide Synthesis}

The production of ceramides within the cell occurs through three major pathways: de novo synthesis, sphingomyelin hydrolysis, and the salvage pathway (Figure 2-5). De novo ceramide synthesis begins with the transfer of a serine residue onto a fatty acyl-CoA via serine palmitoyltransferase (SPT), in a condensation reaction that produces 3-keto-sphinganine (3KSn; Holland and Summers, 2008). In the subsequent reactions, (dihydro)ceramide synthases (CerS) and dihydroceramide desaturases (Des1 and -2), catalyze the conversion of 3KSn into ceramide. Importantly, SPT is highly selective for saturated 16-carbon moieties in several organisms (Holland and Summers, 2008), and it is thus strongly influenced by SFA availability (Merrill, 2002). This explaining why SFA and not unsaturated fats drive the synthesis of sphingolipids (Chavez et al., 2003). In addition, inflammatory signals, such as those produced through the IKK $\beta / \mathrm{NF} \kappa \mathrm{B} / \mathrm{TNF}-\alpha$ axis, upregulate SPT and can thus cause increased ceramide accumulation in adipose tissue (Samad et al., 2006). Moreover, the effects of SFA on the upregulation of ceramide synthesis can be also exerted through activation of the TLRs, involved in innate immune responses. The activation TLRs by SFA results in the production of inflammatory cytokines, such as TNF $\alpha$, and IL-6, resulting in increased ceramide synthesis (Shi et al.; Senn, 2006).

The sphingomyelin hydrolysis pathway generates ceramide in a reaction catalyzed by sphingomyelinase (SMase) enzymes differing in their optimal pH and cellular locations (Deigner et al., 2009; Boulgaropoulos, et al., 2010). Because sphingomyelin is the most abundant sphingolipid in mammals, its hydrolysis has the potential to greatly impact intracellular ceramide concentrations. This pathway is upregulated in obesity through the activation of inflammatory signals (i.e. IKK $\beta / \mathrm{NF} \mathrm{KB} / \mathrm{TNF}-\alpha$ axis), resulting in increased levels of neutral and acid SMases 
(Samad et al., 2006). Importantly, the contribution of this pathway to the accumulation of ceramides in dairy cows has not been established.

Finally, catabolic metabolism of complex sphingolipids contributing to ceramide accrual occurs in late endosomes and lysosomes of cells. The process, often referred to as sphingolipid recycling or salvage (Kitatani et al., 2008), uses acid SMase and acid $\beta$-glucosidase-1 degrade sphingomyelin and glycosphingolipids, respectively, to form ceramide within acidic cellular compartments (Kitatani et al., 2008; Kitatani et al., 2009). Ceramide is then degraded into sphingosine and free fatty acids (FFA), which can then enter the cytosol and be converted back to ceramide via ceramidase activity (Riboni et al., 1998). The pathway can also work in the reverse direction (i.e. ceramide degradation) via acid ceramidase, which deacylates ceramide to produce sphingosine in lysosomes (Kolesnick, 2002). Although the relative importance of the salvage pathway in determining ceramide accumulation during obesity remains unknown, evidence that acid ceramidase overexpression can greatly increase insulin sensitivity, suggests it can potentially impact intracellular ceramide pools (Chavez et al., 2005). 


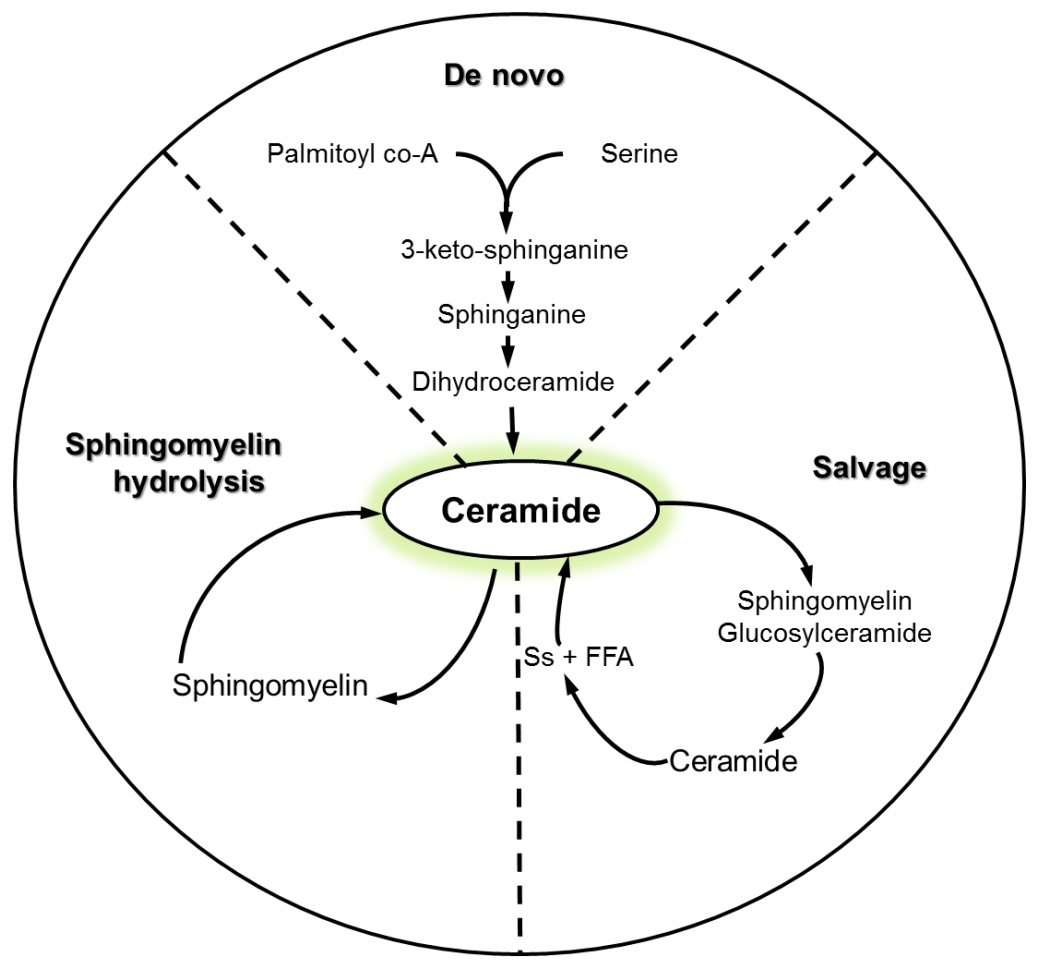

Figure 2-5. Ceramide synthesis and degradation. Main pathways contributing to cellular accumulation of ceramides.

Abbreviations: Sphingosine (Ss), free fatty acid (FFA). Adapted from Bikman and Summers, 2011.

\section{Hepatic Ceramide synthesis and Secretion}

The liver is considered as a primary site of ceramide synthesis, as well as a major contributor of circulating ceramides, responsible for causing systemic insulin resistance and reduced pancreatic insulin secretion (Watt et al., 2012; Boon et al., 2013; Holland and Summer, 2008; Figure 2-6). Similar to other lipids, hydrophobic sphingolipids are transported in the aqueous phase or blood associated with proteins (Hammad et al., 2011). In humans, approximately 98\% of plasma ceramides are found within the apoB-containing low-, and very low-density lipoproteins (LDL and VLDL; Iqbal et al., 2015), as well as in high density lipoproteins (HDL; Lightle et al., 
2003; Boon et al., 2013). The mechanisms of hepatic ceramide assembly and secretion are incompletely understood, however, recent work by Iqbal and collaborators (2015) has provided important insights. Upon synthesis in the ER, ceramides are transported to the Golgi via ceramide transfer protein, four-phosphate adaptor protein 2 (FAPP2), or transport vesicles for the synthesis of sphingomyelin, glycosylated ceramides and other complex sphingolipids. Their works suggest a primordial role of the microsomal triglyceride transfer protein in transferring ceramides and sphingomyelins into nascent apoB-containing lipoproteins (i.e. VLDL). The mechanism by which MTP helps incorporating ceramides into lipoproteins is thus very similar to that used to incorporate TAG, phospholipids (PL) and cholesteryl esters (CE) into VLDL. Interestingly, palmitate has been recently shown to increases cellular MTP levels, thus suggesting that elevated SFA availability can not only upregulate ceramide synthesis, but it also induces its secretion from the liver (Konstantynowicz-Nowicka et al., 2015). 


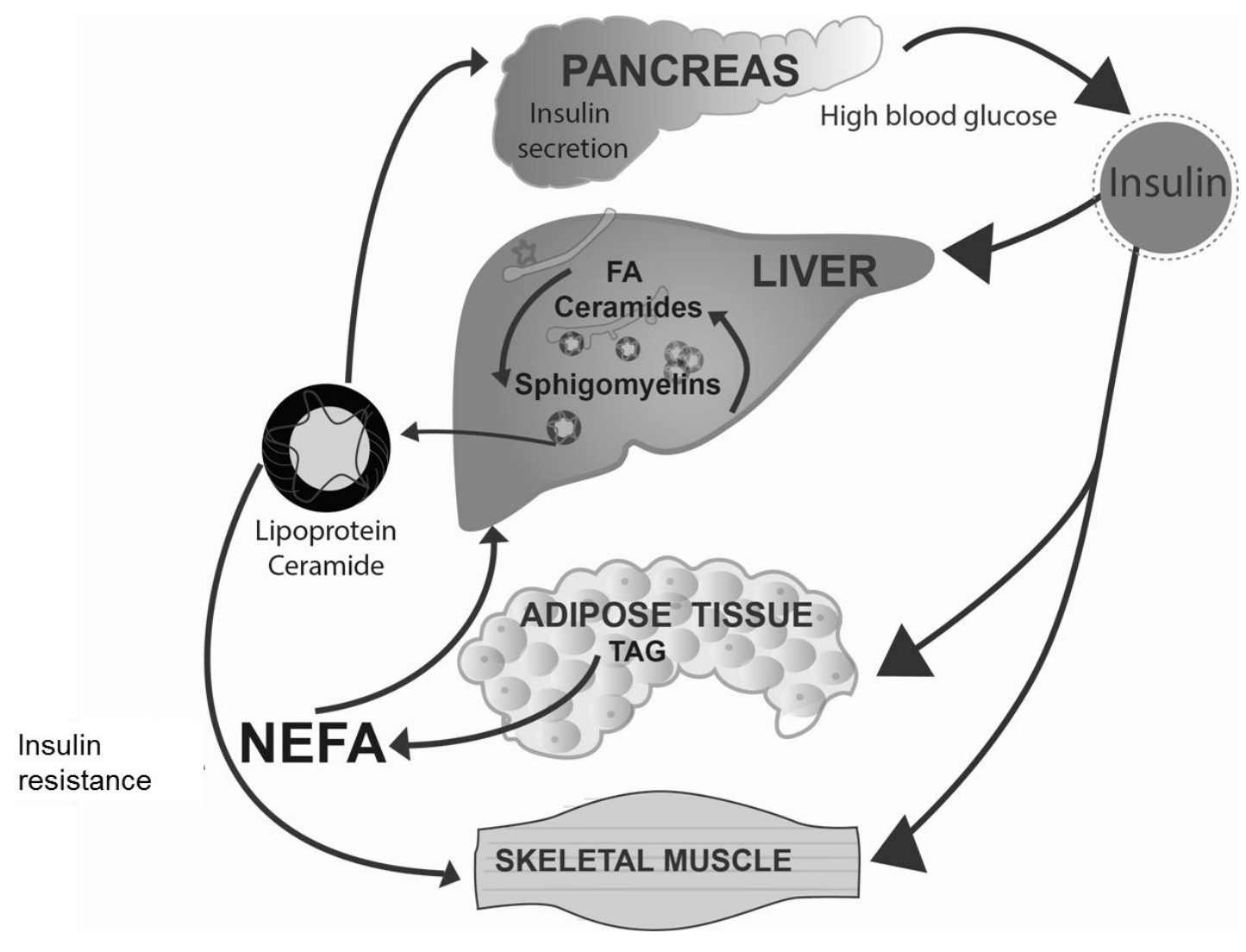

Figure 2-6. Hypothesized model of ceramide synthesis and transport into insulin-producing and insulin-sensing tissues.

\section{Conclusion}

Dairy cows undergo dramatic metabolic adaptations in order to support changing physiological states while preserving appropriate nutrient delivery needs. The transition from gestation to lactation constitutes a period in which the cow's ability to manipulate energy metabolism is challenged, and where the importance of the modulations of insulin action is highlighted. Along with peripartal negative energy balance, which can prolong through early lactation, the possibility of excessive lipomobilization increases, as does the susceptibility for the 
development of metabolic disease. The central role of ceramide in inducing insulin resistance and its association with metabolic disease in monogastrics suggests that it might have comparable importance in dairy cows. Therefore, the objectives of this dissertation are two-fold: first, to investigate the association between circulating ceramides and other sphingolipids with lipomobilization and insulin action during the peripartal period, and during established lactation; and second, to evaluate the potential to manipulate ceramide availability through dietary modifications in order to extend physiological insulin resistance during lactation. 


\title{
Chapter 3 \\ PLASMA CERAMIDES ARE ELEVATED IN OVERWEIGHT HOLSTEIN DAIRY COWS EXPERIENCING GREATER LIPOLYSIS AND INSULIN RESISTANCE DURING THE TRANSITION FROM LATE PREGNANCY TO EARLY LACTATION
}

\begin{abstract}
Insulin resistance is a homeorhetic adaptation to parturition in dairy cows transitioning from late pregnancy to early lactation. An increase in prepartum adiposity can predispose periparturient cows to greater lipolysis and insulin resistance, thus increasing the risk for metabolic disease. Mechanisms mediating the development of insulin resistance in overweight peripartal dairy cows may depend on ceramide metabolism. The sphingolipid ceramide accumulates in plasma and tissues of overweight monogastric animals, and facilitates saturated fatty acid-induced insulin resistance. Considering this evidence, we hypothesized that plasma ceramides would be elevated in periparturient dairy cattle and that these sphingolipids would correlate with the magnitude of lipolysis and insulin resistance. To test our central hypothesis, multiparous Holstein cows were allocated into 2 groups according to their body condition score (BCS) at d -30 prepartum: lean (BCS 4.0; $\mathrm{n}=11$ ). Blood samples were collected at $\mathrm{d}-45,-30,-15$, and -7 , relative to expected parturition, and at d 4 postpartum. Plasma glucose, insulin, nonesterified fatty acids (NEFA), and $\beta$-hydroxybutyrate (BHBA) concentrations were measured, and insulin sensitivity was estimated. The concentrations of individual plasma ceramide and glycosylated ceramide were determined using liquid chromatography-based mass spectrometry. Results demonstrated that greater adiposity was associated with a greater loss in body condition during late pregnancy. Overweight cows had greater circulating concentrations of glucose, insulin, and NEFA, and lower
\end{abstract}


insulin sensitivity relative to lean cows. We detected 30 different sphingolipids across 6 lipid classes with acyl chains ranging from 16 to 26 carbons. The most abundant plasma sphingolipids detected were C24:0-ceramide, C24:0-monohexosylceramide, and C16:0-lactosylceramide. Plasma concentrations of total ceramide and monohexosylceramide increased as lactation approached, and saturated ceramide and monohexosylceramide were elevated in cows with greater adiposity relative to those with a lean phenotype. Plasma ceramides (e.g., C24:0-ceramide) were positively correlated with plasma NEFA and inversely correlated with insulin sensitivity. Our data demonstrate a remodeled plasma sphingolipidome in dairy cows transitioning from late pregnancy to lactation characterized by a concomitant increase in plasma ceramides with the development of peripartal insulin resistance. 


\section{Introduction}

Periparturient dairy cows experience an elevated demand for glucose due to an increased requirement by the mammary gland for lactose synthesis (Bell, 1995). Dairy cattle transitioning from late pregnancy to early lactation develop insulin resistance (Bell and Bauman, 1997) as a metabolic adaptation to energy deficit. Insulin resistance facilitates an increase in hepatic gluconeogenesis and a decrease in glucose uptake by skeletal muscle and adipose tissue (Bell, 1995; Spachmann et al., 2013). Consequently, circulating glucose is spared for lactose synthesis, a major osmotic regulator of milk secretion. To increase NEFA availability for $\beta$-oxidation in peripheral tissues and re-esterification in the mammary gland, the periparturient dairy cow mobilizes triacylglycerol stores in adipose tissue (Drackley et al., 2005; Drackley and Andersen, 2006). Because insulin is an antilipolytic hormone, insulin resistance can further increase adipose tissue lipolysis (Pires et al., 2007a,b). Collectively, the transition dairy cow adapts to energy insufficiency by modifying peripheral insulin action to augment glucose economy. An increase in prepartum adiposity can predispose dairy cows to a greater magnitude of insulin resistance during early lactation (Holtenius et al., 2003; Holtenius and Holtenius, 2007). The greater extent of insulin resistance in overweight peripartal dairy cows can contribute to excessive adipose tissue lipolysis and thus greater metabolic disease risk (e.g., hepatic steatosis and ketosis). Although insulin resistance in dairy cattle transitioning from pregnancy to lactation is mediated in part by growth hormone (Bell and Bauman, 1997), considerable evidence supports a causative role of long-chain saturated fatty acids as antagonists of whole-body insulin sensitivity (Boden, 1997; Funaki, 2009). The ability of surplus saturated fatty acyl-CoA to inhibit insulin action appears to be mediated by the structurally diverse sphingolipid ceramide (Chavez and Summers, 2012). Although ceramides were previously believed to be merely structural elements of cell membranes, recent discoveries 
demonstrate that ceramides are directly implicated in evolutionarily conserved cellular processes, such as cell cycle arrest, apoptosis, and stress responses (Hannun and Obeid, 2008). Ceramides can be (1) formed by de novo synthesis initiated by the condensation of palmitoyl-CoA via action of serine palmitoyltransferase, (2) formed by hydrolysis of sphingomyelin by either acid or neutral sphingomyelinases, or (3) salvaged from complex sphingolipids that are broken down into sphingosine and reacylated (Figure 1; Hirabayashi et al., 2006). An increase in palmitoyl-CoA availability upregulates de novo ceramide synthesis and sphingomyelin hydrolysis in peripheral tissues of obese insulin resistant mice with hepatic steatosis, responses that contribute to ceramide accumulation in tissues and plasma (Cuschieri et al., 2007; Holland et al., 2011). The extent of insulin resistance correlates with the concentrations of ceramide in plasma collected from obese humans with type 2 diabetes, most notably C24:0-ceramide, which is the most abundant ceramide species in human circulation (Haus et al., 2009). Plasma ceramide is transported as components of low-density lipoproteins (LDL) of hepatic origin that contribute to the pathophysiology of wholebody insulin resistance. Although the mechanisms are not completely understood, an increase in the level of LDL enriched with C24:0-ceramide decreases whole-body and skeletal muscle glucose uptake in lean mice, a response accompanied by $\mathrm{C} 24: 0$-ceramide accrual in the plasma membrane and reduced insulin-stimulated protein kinase B phosphorylation (Boon et al., 2013). The effect of endogenous, long-chain plasma ceramide on lipolysis are not known; however, culturing 3T3-L1 adipocytes with exogenous hydrophilic C2:0-ceramide can increase lipolysis by suppressing insulin-stimulated activation of cyclic nucleotide phosphodiesterase 3B (Mei et al., 2002). The inhibitory effect of ceramide on insulin signaling is ameliorated by treatments that decrease $d e$ novo ceramide synthesis (e.g., reducing palmitic acid availability or pharmacological inhibition; Mei et al., 2002; Holland et al., 2007; Ussher et al., 2010). Although the link between NEFA 
mobilization and metabolic disease has been extensively studied (Drackley and Andersen, 2006), the relationship between circulating ceramides, lipolysis, and insulin sensitivity has not been defined. Considering the recognized association between lipotoxicity-induced ceramide accumulation in plasma and the development of insulin resistance in overweight monogastric animals, we hypothesized that plasma ceramide concentrations would increase concomitantly with NEFA availability and the decline in insulin sensitivity characteristic of the ruminant dairy cow transitioning from late pregnancy to early lactation. We further hypothesized that overweight peripartal cows would exhibit greater concentrations of ceramide in plasma and magnitude of insulin resistance compared with lean cows. To test our hypotheses, we used liquid chromatography coupled with mass spectrometry to perform a sphingolipidomic analysis of plasma ceramides and glycosylated ceramides, an approach described by Merrill et al. (2009).

\section{Materials and Methods}

\section{Experimental Design}

Experimental procedures were approved by the West Virginia University Institutional Animal Care and Use Committee (Morgantown). The experiment was completed at Dovan Farms, WVU Agricultural Research and Education Partner, and a 700-Holstein cow commercial dairy farm (located in Berlin, PA). Nonlactating, pregnant, multiparous Holstein cows $(n=21)$ were enrolled in the study $45 \mathrm{~d}$ before expected parturition and allocated to 1 of 2 groups according to their adiposity at $d-30$ relative to calving, as either lean (BCS $2.9 \pm 0.22, n=10)$ or overweight

(BCS $4.1 \pm 0.10, \mathrm{n}=11)$. Parity was not different between BCS groups $(P>0.9)$, averaging $3.5 \pm$ 
0.82 and $3.45 \pm 1.08$ for lean and overweight cows, respectively. Diets were formulated to meet nutrient recommendations and cows were fed once and twice daily during pregnancy and lactation, respectively. All diets were fed for ad libitum intake with free access to water. Ingredient and nutrient compositions of the diets fed during the experiment are shown in Table 3-1. Samples of TMR were obtained weekly throughout the experiment, and DM content was determined by drying at $60^{\circ} \mathrm{C}$ until a static weight was observed. Weekly TMR samples were submitted to a commercial laboratory for nutrient composition analysis by near-infrared spectroscopy (Cumberland Valley, Cumberland, MD; AOAC International, 1995; method 989.03). Body weights and BCS were recorded weekly. Three trained investigators independently recorded BCS for all cows and data were averaged for each cow at $\mathrm{d}-45,-30,-15,-7,4,14$, and 21 relative to expected parturition, postpartum using a 1.0 to 5.0 scale (Wildman et al., 1982). Blood samples (10 mL) were collected by coccygeal venipuncture at $\mathrm{d}-45,-30,-15,-7$, and 4 relative to expected parturition. Blood was kept on ice for $30 \mathrm{~min}$ until centrifugation at $3,400 \times \mathrm{g}$ for $10 \mathrm{~min}$. Following centrifugation, plasma was removed and snap-frozen in liquid nitrogen and then stored at $-80^{\circ} \mathrm{C}$ until further analysis. Milk production was recorded from d 2 to 21 postpartum. Milk samples were collected at d 4 and 10 postpartum, preserved using Bronopol tablets (D\&F Control Systems, San Ramon, $\mathrm{CA}$ ), and stored at $4^{\circ} \mathrm{C}$ for milk component analysis. Plasma samples were analyzed for glucose, insulin, NEFA, BHBA, and ceramides. Plasma concentrations of glucose, NEFA, and BHBA were determined by enzymatic methods using commercial kits (Autokit Glucose, HR Series NEFA-HR (2) and Autokit 3-HB, respectively; Wako Chemicals USA Inc., Richmond, VA). Plasma concentrations of insulin were determined by ELISA using a commercial product (Mercodia Bovine Insulin ELISA; Mercodia AB, Uppsala, Sweden). All spectrophotometric measurements were conducted using a SpectraMax Plus 384 Microplate Reader (Molecular Devices, Sunnyvale, 
CA). Intra- and interassay CV were 3.8 and 3.0\%, 2.6 and 7.7\%, 2.3 and 9.5\%, and 3.7 and $16.6 \%$, for glucose, NEFA, BHBA, and insulin, respectively. Individual milk samples were analyzed for fat, true protein, and lactose concentrations and SCC by mid-infrared spectroscopy (Dairy One, Ithaca, NY; AOAC, 1990; method 972.160) within 1 wk of collection.

\section{Ceramide Measurements}

Plasma extraction was conducted using a modified Bligh and Dyer procedure including ceramide C12:0 as an internal standard (Avanti Polar Lipids, Alabaster, AL; Haughey et al., 2004; Bandaru et al., 2013). Plasma extracts were dried using a nitrogen evaporator and resuspended in pure methanol for analysis. Sample extracts were handled using an autosampler (Leap Technologies Inc., Carrboro, NC) that introduced extracts into an HPLC (PerkinElmer, Boston, MA) with a C18 reverse-phase column (Phenomenex, Torrance, CA). Individual species of ceramide were separated by gradient elution and injected into an electrospray ion source coupled to a triple quadrupole mass spectrometer (API3000; AB Sciex Inc., Thornhill, ON, Canada; Bandaru et al., 2007, 2011, 2013). Ion spray voltage was $5,500 \mathrm{~V}$ at a temperature of $80^{\circ} \mathrm{C}$ with a nebulizer gas of 8 psi, curtain gas 8 psi, and collision gas 4 psi. Declustering potential was $80 \mathrm{~V}$, focusing potential $400 \mathrm{~V}$, entrance potential $10 \mathrm{~V}$, collision energy $30 \mathrm{~V}$, and collision cell exit potential $18 \mathrm{~V}$. Individual ceramide species were detected by multiple reaction monitoring. Sixpoint calibration curves ( 0.1 to $750 \mathrm{ng} / \mathrm{mL}$ ) were constructed by plotting the area under the curve for each calibration standard, C16:0-, C18:0-, C20:0, C22:0-, and C24:0-ceramide, normalized to C12:0- ceramide. Sphingolipid concentrations were determined by curve fitting the identified 
ceramide species based on acyl-chain length. Quantification of spectral data was performed using Analyst 1.4.2 and MultiQuant software (AB Sciex Inc.).

\section{Calculations and Statistical Analysis}

Plasma glucose, insulin, NEFA, and BHBA concentrations were measured, and insulin sensitivity was estimated using the revised quantitative insulin sensitivity check index (RQUICKI; Holtenius and Holtenius, 2007), where RQUICKI = 1/[log (glucose $)+\log ($ insulin $)+\log ($ NEFA) $]$, and glucose $=$ basal glucose $(\mathrm{mg} / \mathrm{dL})$, insulin $=$ basal insulin $(\mu \mathrm{U} / \mathrm{mL})$, and NEFA $=$ basal NEFA (mmol/L), such that lower RQUICKI values suggest reduced whole-body insulin sensitivity, and higher values are indicative of elevated whole-body insulin sensitivity. Changes in BCS and BW of lean and overweight cows were calculated as the differences between values at $\mathrm{d}-21$ and -7 , and the differences between d 4 and 21, relative to parturition. Somatic cell score was calculated from SCC for statistical analysis, using the following logarithmic transformation (Ali and Shook, $1980)$, where $S C S=\log 2(S C C / 100,000)+3$. Changes in BCS and BW pre- and postpartum were analyzed using the GLM procedure of SAS (version 9.3; SAS Institute Inc., Cary, NC). All data for plasma and milk variables were analyzed as repeated measures over time relative to parturition under the MIXED procedure of SAS (SAS Institute Inc.). The statistical model included the random effect of cow nested within BCS and the fixed effects of BCS, day relative to parturition, and their interaction. The most appropriate covariance structure for the repeated-measures analysis was selected for each variable after evaluating 7 different covariance structures (variance components, first-order autoregressive, heterogeneous first-order autoregressive, compound symmetry, heterogeneous compound symmetry, first-order ante-dependence, and unstructured), 
and the structure with the smallest Akaike's information criterion coefficient was selected for analysis. Modeling of the covariance structure allowed the identification of patterns that best describe relationships between the repeated measures in the model. The method of Kenward-Roger was used for calculation of denominator degrees of freedom. Preplanned contrasts were used to evaluate differences between lean and overweight cows at each time point. Relationships between select ceramides were evaluated by random regression, using the MIXED procedure of SAS (SAS Institute Inc.). The full model included the random effect of cow and the linear and quadratic effects of each predictor. Quadratic effects were removed from the model based on fit by Akaike's information criterion scores when $\mathrm{P}>0.05$. Denominator degrees of freedom were calculated by the Satterthwaite method. Studentized residual values $>3.0$ or $<-3.0$ were considered outliers and removed from the analysis (typically 1 per response variable). Nonparametric Spearman rank-

order correlations were performed to determine associations between plasma sphingolipids and NEFA levels, and between sphingolipids and RQUICKI values. All results are expressed as least squares means and their standard errors, unless stated otherwise. Significance was declared at $P<$ 0.05 and trends at $P<0.10$.

\section{Results and Discussion}

Overweight dairy cows transitioning from late pregnancy to early lactation will mobilize more adipose to meet energy demands than lean cows (Rukkwamsuk et al., 1999). To validate this response, BCS and BW were evaluated in lean and overweight transition cows (Figure 3-2). Cows that were overweight experienced accelerated BCS loss $(P<0.01)$ and tended to lose more BW $(P$ $=0.10$ ) during late pregnancy; however, no differences in the rate of BCS or BW loss were detected 
during lactation (data not shown). Milk yield increased during early lactation but was not affected by BCS $(29.4 \pm 1.2,37.5 \pm 1.0,39.3 \pm 1.0$ and $42.1 \pm 1.1 \mathrm{~kg} / \mathrm{d}$, for $4,10,14$ and 21 DIM, respectively; $P<0.001)$. Although no change was observed for milk fat or lactose yield, a tendency for a BCS $\times$ day relative to parturition interaction was found for increased milk protein yield in overweight cows at 10 DIM (1.38 vs. $1.15 \mathrm{~kg} / \mathrm{d} ; P=0.06)$. No significant response was detected for SCS; however, overweight cows tended to have an elevated SCS at 10 DIM relative to lean cows ( 2.27 vs. 1.86 , respectively; $P=0.10$ ) - a potential indicator of compromised mammary gland immune function. In overweight humans, elevated plasma concentrations of glucose, insulin, and NEFA are markers associated with the development of insulin resistance, hallmark characteristics of type 2 diabetes (Reaven et al., 1988; Thévenod, 2008). Comparably, Holtenius and Holtenius (2007) demonstrated a negative linear relationship between adiposity and RQUICKI (i.e., cows with a high BCS have a low RQUICKI value), an observation supported by reduced glucose disappearance following a glucose tolerance test in overweight cows (Holtenius et al., 2003; Jaakson et al., 2013). For validation, we measured the plasma concentrations of glucose, insulin, and NEFA, and calculated RQUICKI values for lean and overweight cows during the transition from pregnancy to lactation (Figure 3-3). Compared with lean cows, plasma glucose concentrations were greater in overweight animals at d -15 and -7 relative to parturition $(P<0.05)$. Insulin concentrations were higher in overweight cows at d -30 and -15 $(P<0.01)$, and tended to be greater at $\mathrm{d} 4(P=0.06)$. A progressive decline in plasma insulin concentrations was observed, being 25 and $55 \%$ lower at $d-7$ and 4 , respectively, relative to $d-30(P<0.001)$. Plasma NEFA concentrations increased by 30,149 , and $321 \%$ on $\mathrm{d}-15,-7$ and 4 , respectively, relative to $\mathrm{d}-30$ $(P<0.001)$. As expected, overweight cows had higher NEFA concentrations at d $-15(P<0.05)$, and tended to be higher at $\mathrm{d}-7$ and 4 relative to parturition $(P=0.06)$. The observed increase in 
plasma NEFA and decrease in plasma insulin at the onset of lactation are characteristic homeorhetic adaptations to maximize milk synthesis, responses linked with lower voluntary energy intake (Drackley et al., 2005). These physiological changes were associated with lower RQUICKI values postpartum $(P<0.001)$, indicative of reduced insulin sensitivity. Specifically, RQUICKI decreased by 8,15 , and $14 \%$ on $\mathrm{d}-15,-7$, and 4 , respectively, relative to $\mathrm{d}-30(P<$ 0.001). Compared with lean cows, insulin sensitivity was significantly lower at d $-30,-15,-7$ and 4 relative to parturition $(P<0.05)$. We also observed lower RQUICKI values in dairy cattle with greater adiposity as early as $\mathrm{d}-45(P=0.07)$, a pathological response that preceded prepartum elevations in plasma glucose, insulin, and NEFA. The observed reductions in prepartum insulin sensitivity may relate to antagonism of adipose tissue insulin signal transduction by saturated fatty acids in overweight cows (Boden, 1997), an action that could explain the observed acceleration in adipose tissue NEFA mobilization. Plasma concentrations of BHBA were not affected by BCS; however, BHBA was $40 \%$ higher at d 4 relative to d -30 (465 vs. $333 \mu \mathrm{mol} / \mathrm{L}$, respectively; $P<$ 0.01). The acyl chain specificity of 6 dihydroceramide synthases produces structurally diverse sphingolipids (Park and Pewzner-Jung., 2013). To elucidate the structural diversity of ceramide and glycosylated ceramide in dairy cows, we used liquid chromatography coupled with mass spectrometry to separate 30 plasma species by acyl chain length including long-chain ceramides, monohexosylceramides, and lactosylceramides, as well as a limited number of dihydro species (Figure 3-4). Similar to humans and rodents (Ichi et al., 2006; Haus et al., 2009), C16:0- and C24:0-ceramide were the most abundant plasma ceramides, representing 30 and $58 \%$ of total ceramide, respectively, across lean and overweight cows. Similarly, C24:0-monohexosylceramide and C16:0-lactosylceramide represented the major plasma monohexosylceramide and lactosylceramide species, respectively. In humans, approximately $98 \%$ of plasma ceramides are 
found within liver-derived lipoprotein subfractions (i.e., high, low, and very low density lipoproteins; Lightle et al., 2003; Boon et al., 2013); however, the origin of ceramides over time and between BCS groups in this study is unknown and will require further definition. Measurement of plasma ceramides can be a means to diagnose the severity of insulin resistance in humans experiencing hyperlipidemia and nonalcoholic fatty liver disease (Haus et al., 2009). Therefore, we compared the plasma ceramide and glycosylated ceramide profiles with the development of insulin resistance in lean and overweight dairy cows (Figure 3-5). Plasma concentrations of total ceramide increased as parturition approached (Figure 3-6); however, these responses were accelerated in dairy cows exhibiting greater adiposity, NEFA mobilization, and magnitude of insulin resistance. Of interest, plasma C24:0-ceramide, the most abundant ceramide in bovine plasma, increased by $92 \%$ on $\mathrm{d} 4$ relative to $\mathrm{d}-30$ ( $\mathrm{P}<0.001$; Figure 7$)$, and tended to be greater in overweight cows at d -7 $(P=0.09)$. Concentrations of C18:0-, C18:1-, C20:0-, C22:0, C22:1, and C24:1-ceramide were also significantly greater in overweight cows during the peripartal period $(P<0.05$; Figure 3-10), relative to lean cows, and C26:0- and C26:1-ceramide concentrations tended to be increased in overweight dairy cows on $\mathrm{d} 4$ and $\mathrm{d}-15$, respectively, relative to d $-30(P=0.06)$. In contrast, plasma $\mathrm{C} 16: 0$-ceramide concentrations did not change with time or score of adiposity (Figure 4-7). Because palmitic acid is a required substrate for $d e$ novo synthesis of all sphingolipids including ceramides, glycosylated ceramides, and sphingomyelins (i.e., palmitic acid is part of the sphingoid backbone), palmitic acid availability may have been limited for C16:0-ceramide synthesis in overweight cows. De novo synthesis of ceramide is contingent on the fatty acyl-CoA-dependent acylation of sphinganine to form dihydroceramide, a reaction mediated by dihydroceramide synthase. We measured one dihydroceramide in bovine plasma, C16:0-dihydroceramide (Figure 3-11). Plasma C16:0- 
dihydroceramide tended to be greater in overweight cows at $\mathrm{d}-15(+47 \% ; P=0.07)$. The elevated concentration of plasma C16:0-dihydroceramide is indicative of increased ceramide anabolism in tissues of dairy cows transitioning from pregnancy to lactation. We also detected a significant positive association between plasma C16:0-dihydroceramide and C16:0-ceramide across all sampled cows $(P<0.001$; Figure 12). The strong relationship between these sphingolipids may be explained by enhanced activity of hepatic dihydroceramide desaturase in transition dairy cows; however, additional research is needed to confirm this hypothesis. Glycosylated ceramides include monohexosylceramides (e.g., glucosylceramide and galactosylceramide) and lactosylceramides, glycosphingolipids synthesized from ceramide and precursors for monosialodihexosylganglioside synthesis (GM3, a ganglioside; Figure 3-1). Similar to ceramide, monohexosylceramide levels are elevated in plasma from obese, insulin-resistant rodents (Chavez et al., 2014). To determine whether a similar relationship exists in overweight dairy cows, we profiled these plasma glycosphingolipids (Figures 3-5, 3-6, and 3-7 and Figure 3-13 and Figure 3-14). Subsequent to the prepartum increases in plasma ceramides, we detected an increase in the concentrations of most plasma monohexosylceramides in overweight cows postpartum (Figure 5). These results were expected because monohexosylceramide synthesis is contingent on ceramide availability (Figure 1). For example, as plasma NEFA and ceramide levels increased with time, the concentration of C24:0-monohexosylceramide (the most hexosylceramide synthesis is contingent on ceramide availability (Figure 3-1). For example, as plasma NEFA and ceramide levels increased with time, the concentration of C24:0-monohexosylceramide (the most abundant plasma monohexosylceramide) increased $(P<0.001$; Figure 3-7). Relative to adiposity, plasma C24:0monohexosylceramide was elevated in overweight cows at d 4 postpartum $(\mathrm{BCS} \times$ day relative to parturition, $P<0.01)$. Similar observations were seen for C24:0-lactosylceramide. Furthermore, 
we observed a strong positive association between C24:0-ceramide and C24:0monohexosylceramide, and between C24:0- monohexosylceramide and C24:0-lactosylceramide across all sampled cows (Figure 3-8). These results may indicate a coordinated regulation of synthesis for these sphingolipids containing the C24:0 acyl moiety in tran sition dairy cow tissues. In contrast to total ceramide and monohexosylceramide, total lactosylceramide concentrations were not modified with enhanced adiposity (Figure 6); however, C16:0-lactosylceramide (the most abundant plasma lactosylceramide) concentrations were lower in overweight cows compared with lean cows $(P<0.01)$. Increased circulating NEFA can promote insulin resistance by promoting ceramide accumulation in plasma and peripheral tissues of humans and rodents (Watt et al., 2012). Considering that NEFA can induce insulin resistance in dairy cows (Pires et al., 2007a), we hypothesized that plasma ceramide concentrations would increase concomitantly with NEFA availability and the development of insulin resistance. To test this hypothesis, we performed a Spearman correlation analysis to identify significant correlations between individual sphingolipid species, and NEFA levels or RQUICKI values (Figure 3-9). As expected, NEFA correlated negatively with insulin sensitivity $(r=-0.62, P<0.05)$. The majority of ceramides and monohexosylceramides were positively correlated with NEFA concentrations and negatively correlated with insulin sensitivity. Significant positive correlation coefficients between NEFA levels and multiple ceramide species were detected, ranging from 0.23 to $0.60(P<0.05)$, with the strongest correlations observed for C24:0-ceramide, total monohexosylceramide, and C22:0monohexosylceramide $(P<0.001)$. Significant negative correlation coefficients between ceramides and RQUICKI values ranged from -0.33 to $-0.22(P<0.05)$, and included total ceramide as well as individual ceramide subspecies (i.e., 20:0, C22:0, C24:0, C24:1, and C26:0). A remarkably similar relationship has been observed in obese type 2 diabetic patients and lean 
healthy control subjects (Haus et al., 2009). In further support, baseline measurements of C20:0-, C24:0-, and C24:1-ceramide were inversely related to insulin sensitivity in overweight adults (Dubé et al., 2011). In contrast, we did not detect significant correlations between plasma glycosylated ceramides (i.e., monohexosylceramides or lactosylceramides) and estimated wholebody insulin sensitivity. Comparably, dihydroceramide and ceramide classes correlate with type 2 diabetes and prediabetes in humans, correlations not observed for monohexosylceramide or lactosylceramide (Meikle et al., 2013). The development of insulin resistance and lipolysis represent coordinated shifts in metabolism to support lactation. Reduced insulin stimulation during the transition from gestation to lactation allows adipose tissue metabolism to change from lipogenic to lipolytic (McNamara, 1991), a shift that facilitates hepatic NEFA processing (Drackley et al., 2005). Although ceramides can be synthesized from NEFA and are known effectors of insulin resistance in monogastrics, little is known of their involvement in the homeorhetic process that modifies nutrient partitioning in cows transitioning from gestation to lactation. Because our observed increases in plasma ceramide concentrations occurred concomitantly with changes in NEFA, insulin, and insulin sensitivity in both lean and overweight cows during the transition from gestation to lactation (Figure 3-3, Figure 3-6, and Figure 3-9), the accumulation of ceramide may represent a homeorhetic adaptation that allows periparturient cows to undergo these characteristic shifts in metabolism. Given that the mammalian sphingolipidome comprises a vast array of complex lipids, a major challenge in the field of sphingolipid biology is to identify which species are the primary effectors of insulin resistance in different tissues. A limitation of the current study is that we did not evaluate ceramide metabolism or insulin signaling in liver, skeletal muscle, or adipose tissue. Future studies will need to use rapidly advancing 
sphingolipidomics-based methodologies to address whether plasma or tissue ceramide can mediate the development of insulin resistance in periparturient dairy cows.

\section{Conclusion}

Insulin resistance accelerates lipolysis and increases metabolic disease risk in periparturient dairy cattle, and ceramide is an inhibitor of insulin action in monogastrics; however, the relationship between plasma ceramide and insulin resistance has not been characterized in peripartal cows. We conclude that ceramides accumulate in plasma during the progression of insulin resistance in overweight cows transitioning from late pregnancy to early lactation. These data support the potential involvement of ceramide in the pathological development of insulin resistance in dairy cattle. We also conclude that changes in plasma ceramide abundance occur concomitantly with changes in plasma NEFA, insulin, and insulin sensitivity, suggesting that circulating ceramides may be fundamentally involved in the homeorhetic adaptation to early lactation. To evaluate these possibilities, future work should focus on determining whether augmented insulin resistance in overweight cows is caused by maladaptive changes in fatty acid processing that promote the synthesis and accumulation of ceramide, and whether antagonism of insulin signaling is ceramide dependent. Identifying the mechanisms of insulin resistance may reveal metabolic pathways that can be manipulated to improve insulin sensitivity, reduce lipolysis, and improve cow health. Researchers should also evaluate the efficacy of plasma ceramide subspecies as diagnostic predictors for metabolic disease risk relative to the commonly utilized biomarkers NEFA and BHBA. 
Table 3-1. Ingredients and nutrient composition of diets fed to lean and overweight cows during the transition from late pregnancy to early lactation.

\begin{tabular}{|c|c|c|c|}
\hline \multirow[b]{2}{*}{ Ingredients, \% of DM } & \multicolumn{3}{|c|}{ Diets } \\
\hline & d -60 to -21 & $\begin{array}{c}\mathrm{d}-21 \text { to } \\
\text { parturition }\end{array}$ & Lactation \\
\hline Corn silage & 32.2 & 30.7 & 42.4 \\
\hline Mixed grass haylage & 29.8 & 28.4 & 7.0 \\
\hline Mixed grass hay & 13.9 & 13.2 & 2.1 \\
\hline Dry ground corn & & - & 14.4 \\
\hline Prepartum mix ${ }^{1}$ & 14.7 & 13.9 & - \\
\hline Lactation mix $\mathrm{A}^{2}$ & - & - & 14 \\
\hline Aminoplus & 9.4 & 9.0 & 4.7 \\
\hline Cottonseed with lint & - & - & 4.5 \\
\hline Sugar cane syrup & - & - & 3.6 \\
\hline Lactation mix $\mathrm{B}^{3}$ & - & - & 4.5 \\
\hline Close-up supplement ${ }^{4}$ & - & 4.8 & 2.7 \\
\hline Rumensin ${ }^{5}$ & - & - & 0.1 \\
\hline \multicolumn{4}{|l|}{ Nutrient Composition } \\
\hline $\mathrm{DM}, \%$ & 49.1 & 49 & 47.4 \\
\hline $\mathrm{NDF}, \%$ of $\mathrm{DM}$ & 48 & 49 & 33.4 \\
\hline Forage NDF, \% of DM & 42.8 & 43.7 & 25.1 \\
\hline Forage NDF, $\%$ of NDF & 89.2 & 89.2 & 75.3 \\
\hline $\mathrm{ADF}, \%$ of $\mathrm{DM}$ & 32 & 32.5 & 21.4 \\
\hline $\mathrm{CP}, \%$ of $\mathrm{DM}$ & 12.3 & 12.2 & 16.5 \\
\hline Starch, \% of DM & 15 & 14.7 & 24.8 \\
\hline Crude fat, $\%$ of DM & 3.1 & 3.4 & 4.4 \\
\hline Ash, \% of DM & 7.4 & 7.5 & 7.6 \\
\hline
\end{tabular}

${ }^{1}$ Mix contained 27\% commercial dry cow mix with Animate (Phibro Animal Health Corp., Teaneck, NJ), $17.3 \%$ ground corn, $17.3 \%$ crimped oats, $13 \%$ corn distillers, $12.9 \%$ soybean meal, $4.3 \%$ calcium carbonate, $4.3 \%$ calcium sulfate, 2.6\% Omnigen AF (Phibro Animal Health Corp.), 1.1 Monocalcium phosphate, and < $1 \%$ of each of the following: Sel-plex 600 (Alltech Biotechnology, Nicholasville, KY) and vitamin E. ${ }^{2}$ Mix contained $33.6 \%$ citrus pulp, $19 \%$ soybean meal. $15.6 \%$ canola meal, $14 \%$ soybean hulls, $4.5 \%$ calcium carbonate, $4.5 \%$ sodium bicarbonate, $2.8 \%$ urea, $2.7 \%$ fat, $2.4 \%$ sodium chloride, and $<1 \%$ from each of the following: Monocalcium phosphate, biotin, and Rumensin 90 (Elanco Animal Health, Greenfield, IN). ${ }^{3}$ Mix contained 43.6\% Fermenten (Church and Dwight Co., Princeton, NJ), $21.8 \%$ calcium carbonate, $10.8 \%$ soybean hulls, $7.8 \%$ Mintrex blend (Novus International Inc., St. Charles, MO), 5.2\% blood meal, 3.5\% magnesium oxide, 2.6\% Celmanax (Vi-COR, Mason City, IA), $2.6 \%$ Omnigen (Phibro Animal Health Corp.), and $<1 \%$ of each of the following: vitamin E, selenium selenite, and selenium yeast $600 .{ }^{4} \mathrm{Mix}$ contained $74.5 \%$ ground oats, $15.3 \%$ commercial amino acid, $8 \%$ Reashure (Balchem Encapsulates, Slate Hill, NY), and $<1 \%$ of each of the following: vitamin E and Niashure (Balchem Corporation, New Hampton, NY). ${ }^{5}$ Rumensin for dairy included at $4,890 \mathrm{mg} / \mathrm{kg}$. 


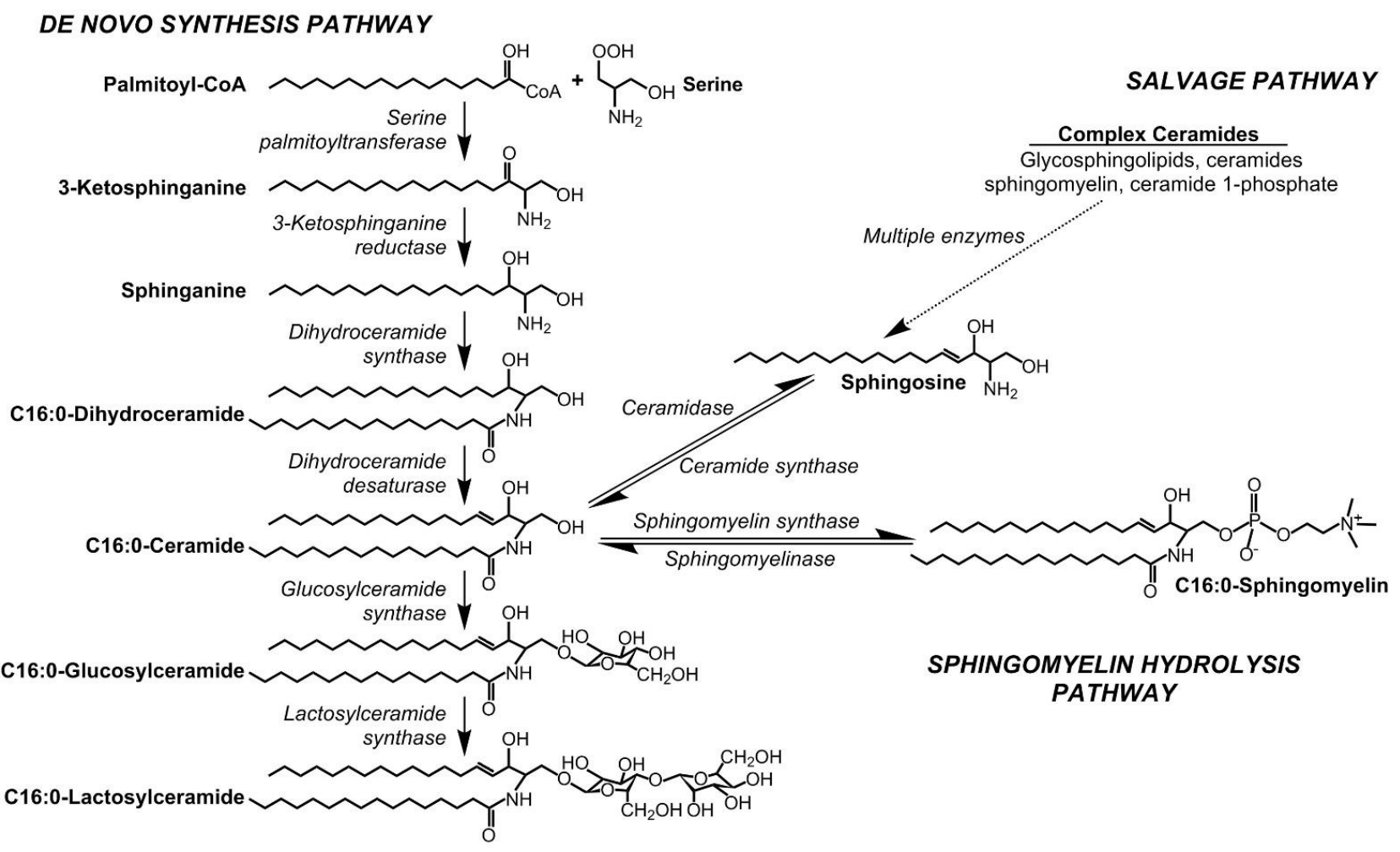

Figure 3-1. Metabolic pathways of ceramide synthesis.

Ceramide can be generated by three pathways: de novo synthesis initiated by the condensation of palmitoyl-CoA and serine to form 3-ketosphinganine, hydrolysis of sphingomyelin, or breakdown of complex sphingolipids via a series of reactions referred to as the salvage pathway. Complex sphingolipids have various acyl chain lengths varying in carbon length and degree of saturation. For simplicity, C16:0-linked sphingolipids and glycosphingolipids are illustrated. Monohexosylceramides consist of a single sugar residue, either glucose or galactose (galactosylceramide is not shown). 


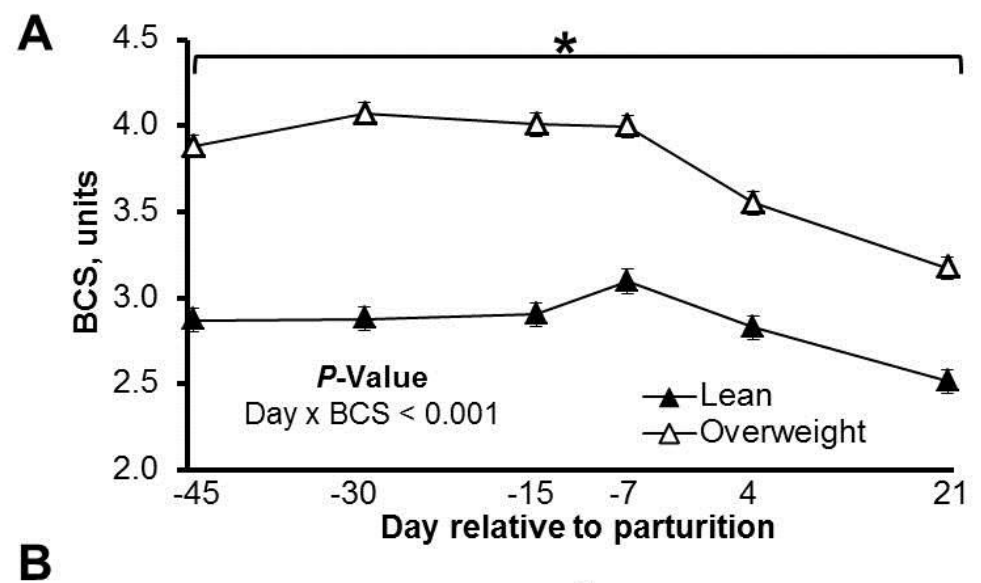

B
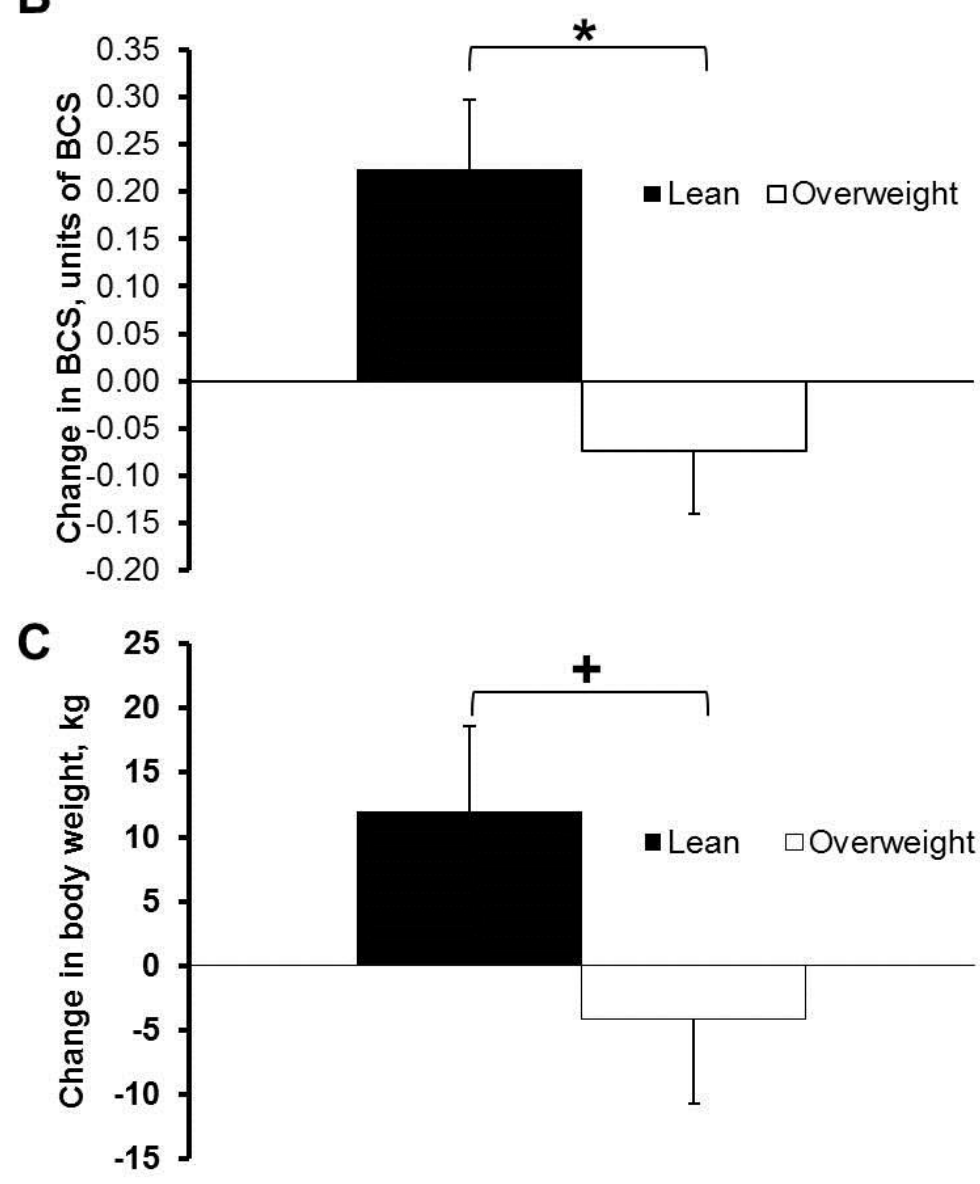

Figure 3-2. Overweight dairy cows lost more prepartum body condition and weight, relative to lean cows. 

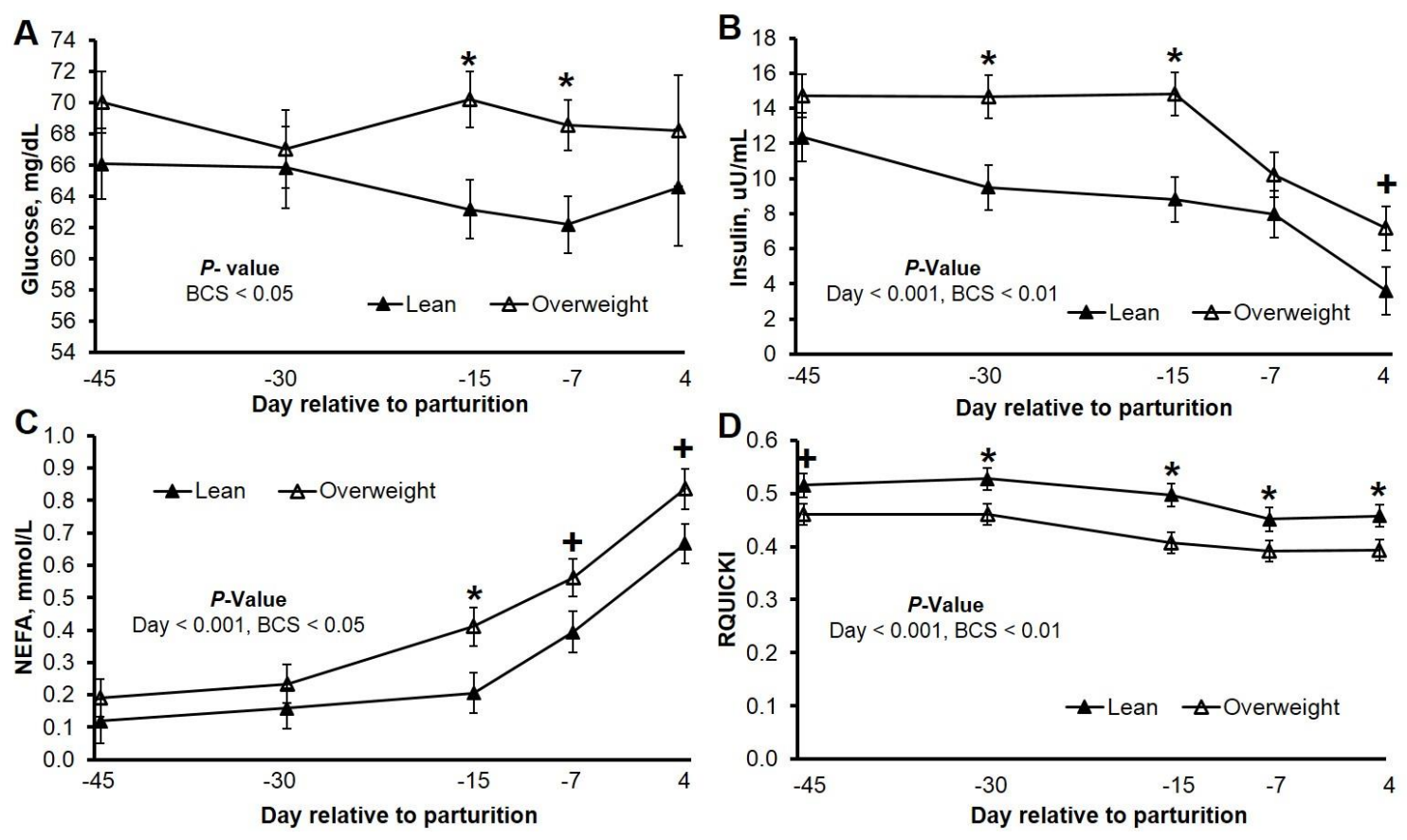

Figure 3-3. Overweight dairy cows are insulin resistant before onset of lipolysis.

Plasma concentrations of (A) glucose, (B) insulin, and (C) NEFA, of lean and overweight dairy cattle transitioning from late pregnancy to early lactation. (D) Estimated insulin sensitivity as measured by the revised quantitative insulin sensitivity check (RQUICKI). RQUICKI was calculated as follows: $1 /[\log ($ glucose $)+\log ($ insulin $)+\log (\mathrm{NEFA})]$. Lower RQUICKI values are indicative of reduced insulin sensitivity. Data are least squares means and their standard errors. *, $P<0.05 ;+, P<0.10$. 

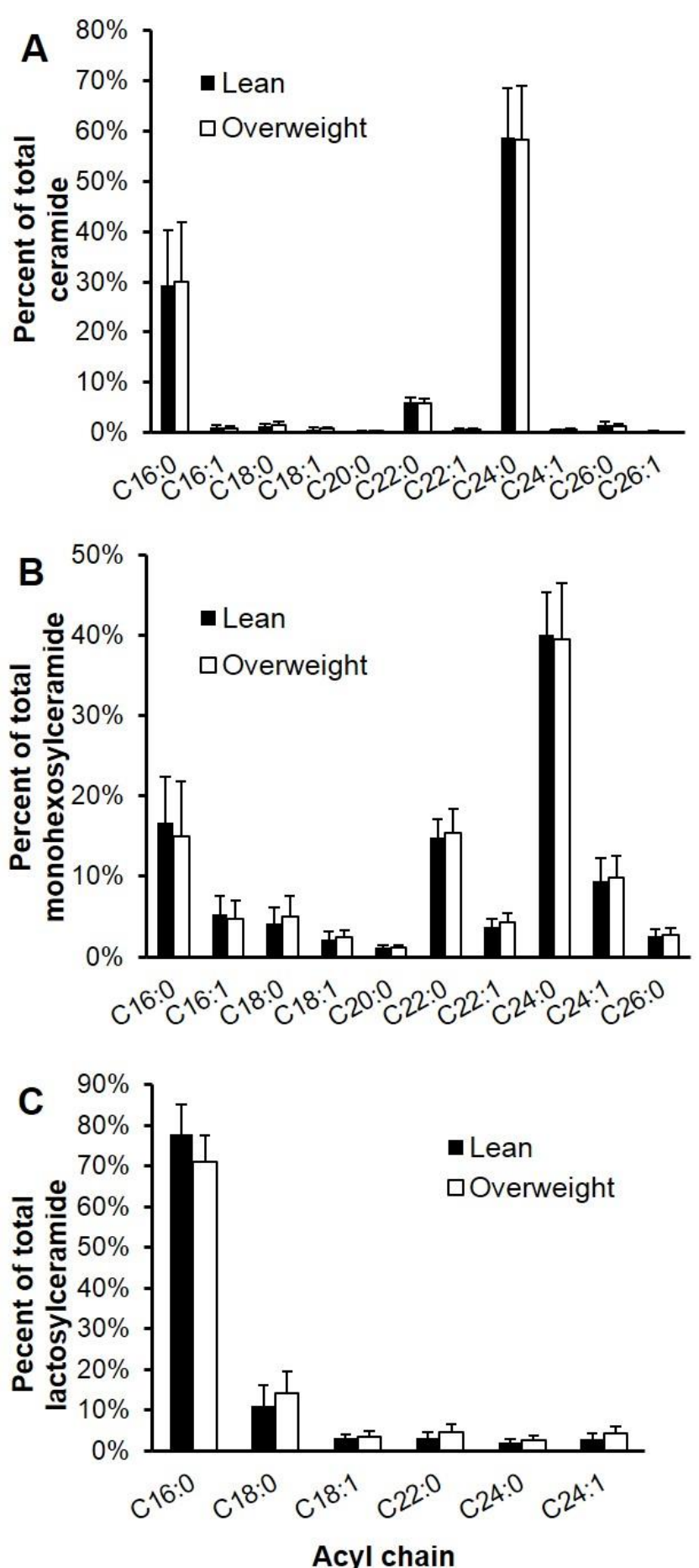

Figure 3-4. C16:0- and C24:0-ceramide are high-abundant ceramides in bovine plasma.

Plasma composition of (A) ceramides, (B) monohexosylceramides, and (C) lactosylceramides in lean and overweight dairy cows transitioning from late pregnancy to early lactation (d -30 to 4, relative to parturition). C16:0-dihydroceramide, C16:0-dihydro-monohexosylceramide, and C16:0-dihydro-lactosylceramide are not included. Data presented as mean abundance \pm SD. 


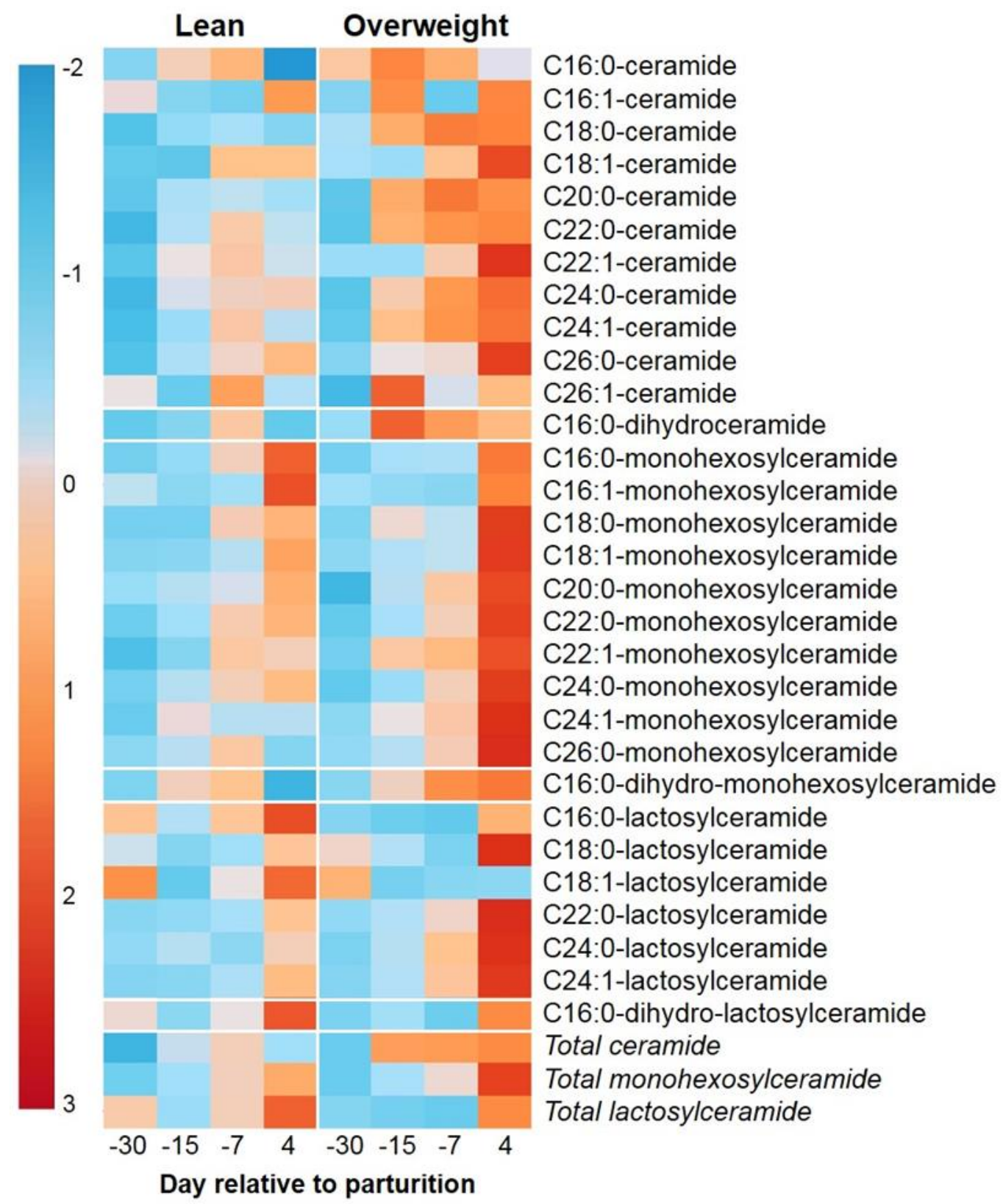

Figure 3-5. Plasma ceramides, monohexosylceramides, and lactosylceramides are elevated in overweight dairy cows transitioning from gestation to lactation.

For visualization purposes, the heat map represents the magnitude of mean-centered, fold change increases (red) or decreases (blue) for each sphingolipid or glycosphingolipid, relative to lean cows sampled at d -30 prepartum. The heat map was generated using MetaboAnalyst 2.0 (Xia et al., 2012). 

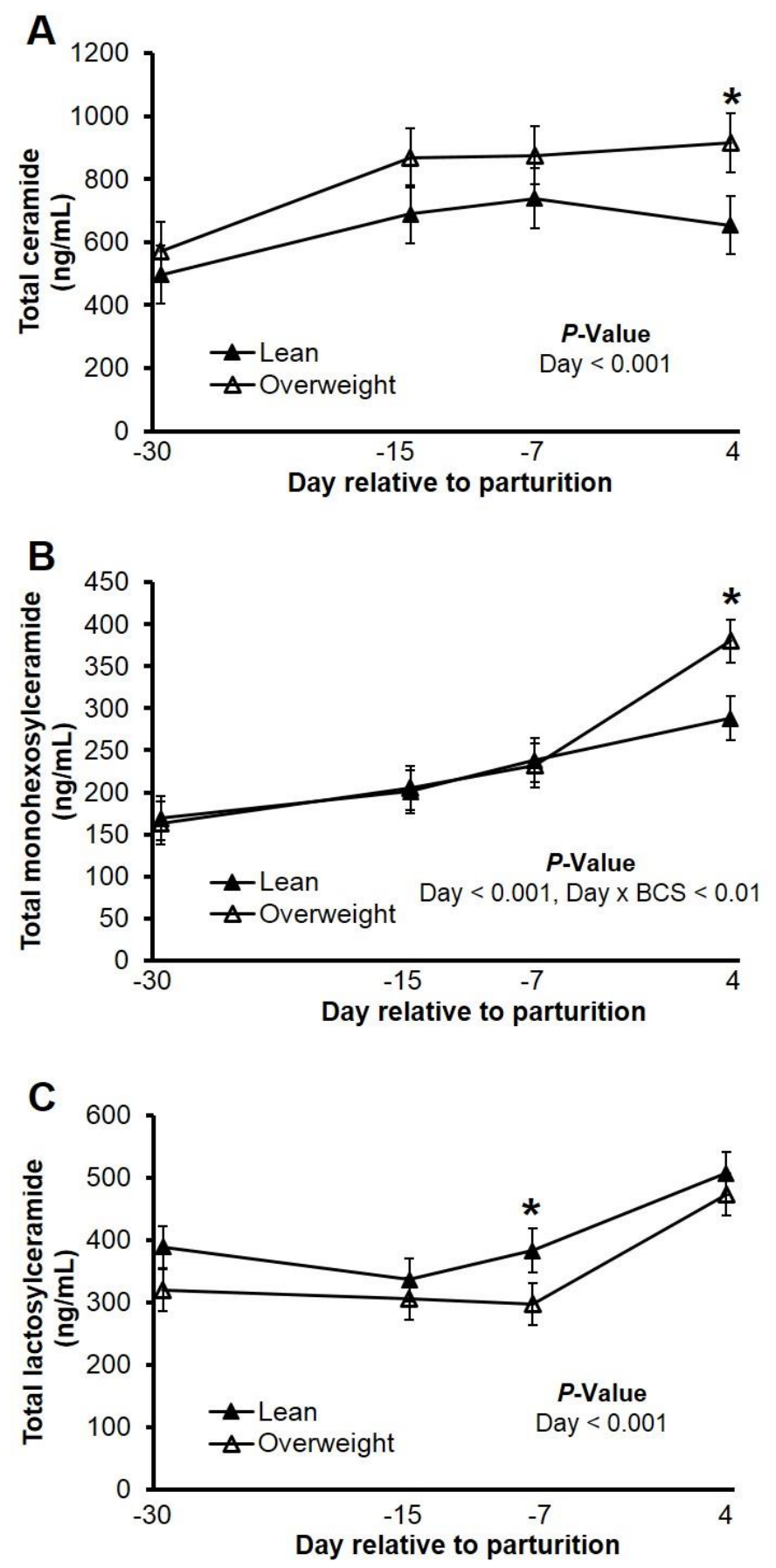

Figure 3-6. Plasma concentration $(\mathrm{ng} / \mathrm{mL})$ of total ceramides, monohexosylceramides, and lactosylceramides in lean and overweight cows transitioning from late pregnancy to early lactation, relative to lean cows.

Plasma concentration of total (A) ceramide, (B) monohexosylceramide, and (C) lactosylceramide in lean and overweight peripartal dairy cows. Data are represented as least squares means and their standard errors. *, $P<0.05 ;+, P<0.10$. 

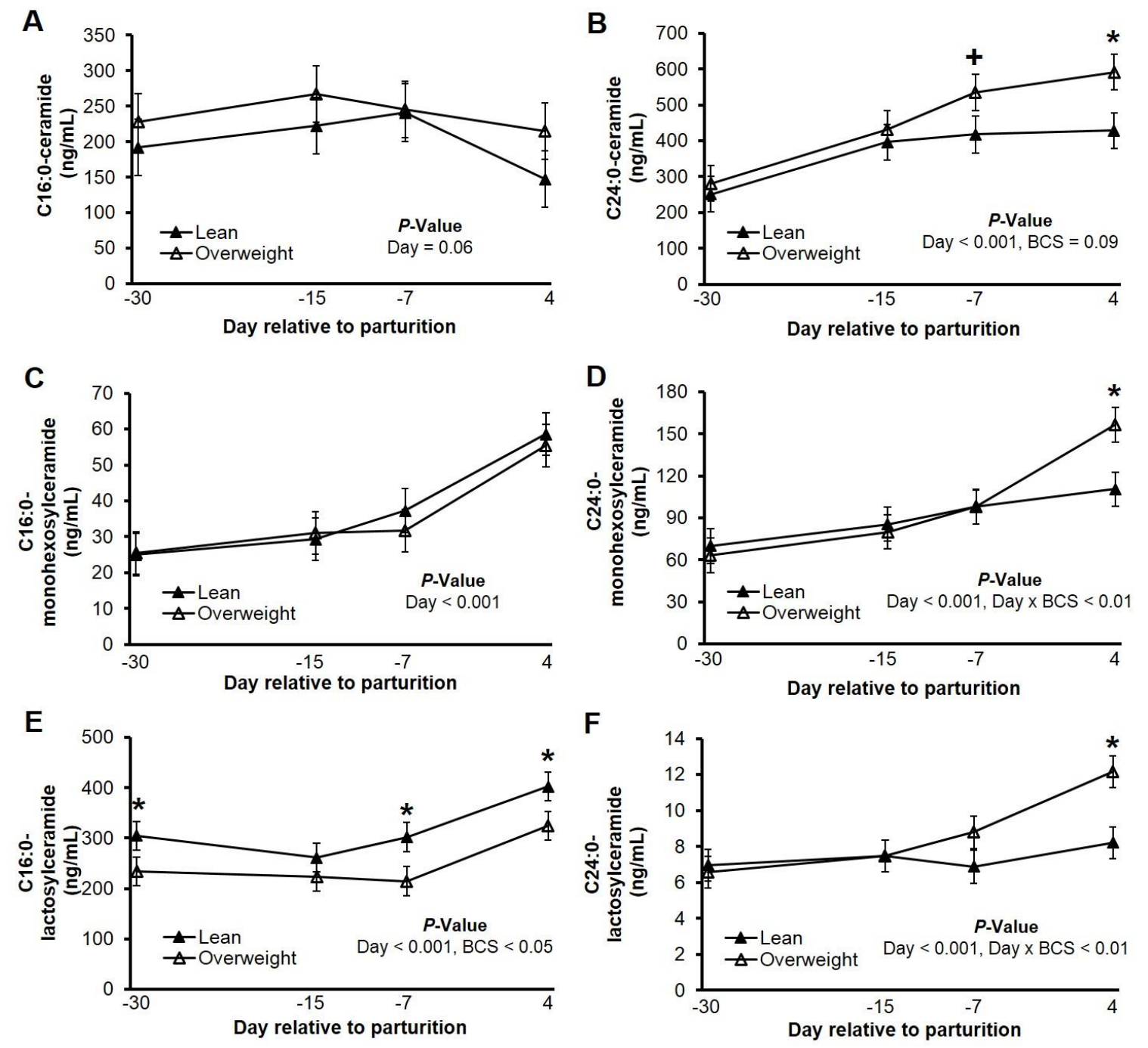

Figure 3-7. Plasma concentration (ng/mL) of C24:0-linked ceramide, monohexosylceramide, and lactosylceramide is elevated in overweight cows transitioning from late pregnancy to early lactation, relative to lean cows.

Plasma concentration of (A) C16:0-ceramide, (B) C24:0-ceramide, (C) C16:0monohexosylceramides, (D) C24:0-monohexosylceramide, (E) C16:0-lactosylceramide, and (F) C24:0-lactosylceramide in lean and overweight transition dairy cows. Data are represented as least squares means and their standard errors. ${ }^{*}, P<0.05 ;+, P<0.10$. 

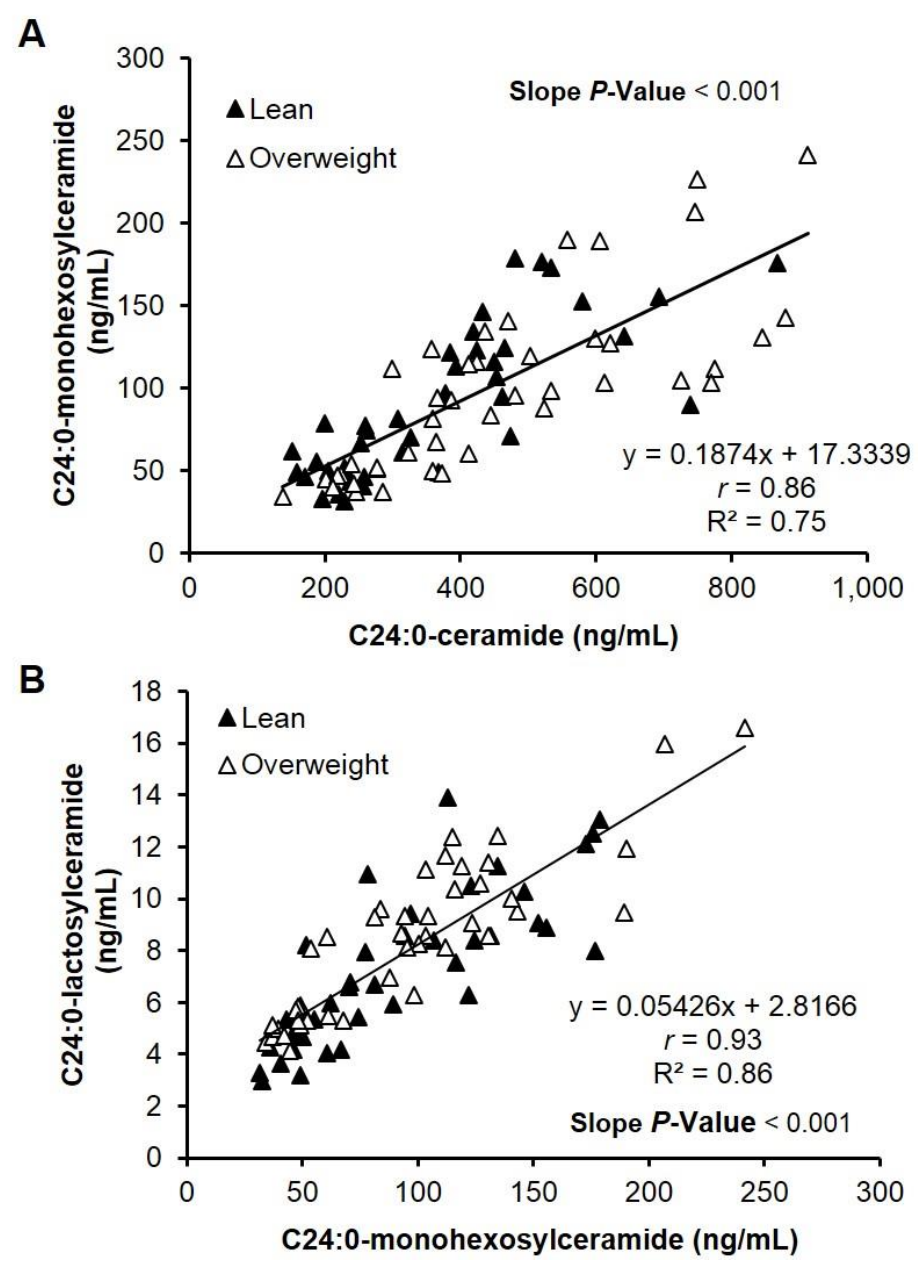

Figure 3-8. Plasma C24:0-ceramide is positively associated with C24:0-monohexosylceramide, and C24:0-monohexosylceramide is positively associated with C24:0-lactosylceramide.

Random regression analysis between plasma concentrations of (A) C24:0-ceramide and C24:0monohexosylceramide and (B) C24:0-monohexosylceramide and C24:0-lactosylceramide in lean and overweight dairy cows during the transition from late pregnancy to early lactation. $\mathrm{R}^{2}$ coefficients represent the association between observed and model predicted values when accounting for the random effect of cow. Data represents measurements for $\mathrm{d}-30,-15,-7$, and 4 , relative to parturition. 


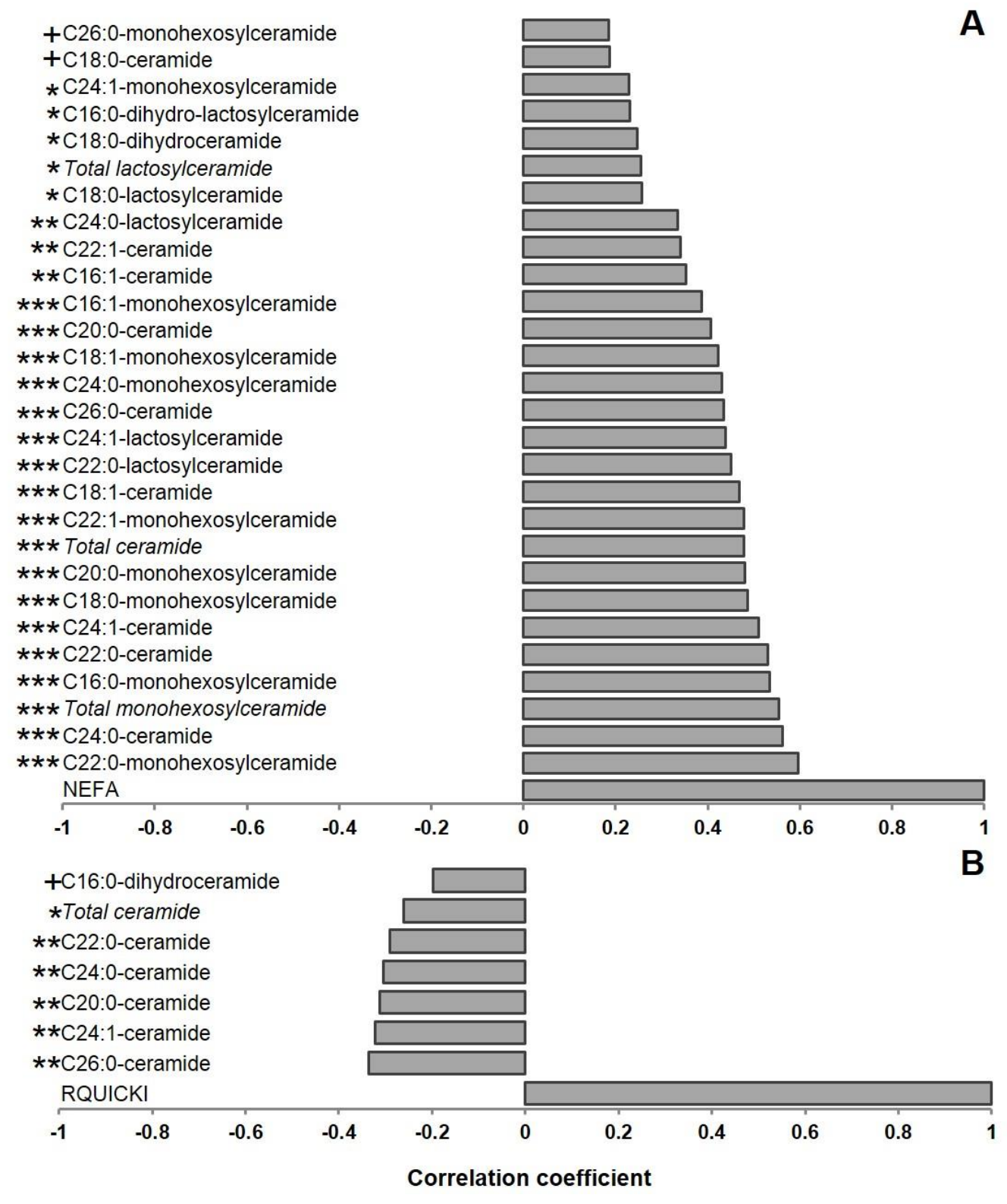

Figure 3-9. Plasma ceramides are positively correlated with circulating NEFA, and negatively correlated with insulin sensitivity in dairy cows transitioning from late pregnancy to lactation.

Non-parametric Spearman's rank-order correlations of circulating ceramides to (A) plasma NEFA and (B) revised quantitative insulin sensitivity check (RQUICKI). RQUICKI was calculated as follows: $1 /[\log ($ glucose $)+\log ($ insulin $)+\log ($ NEFA $)]$. Lower RQUICKI values are indicative of reduced insulin sensitivity. ${ }^{* *}, P<0.001 ; * *, P<0.01 ; *, P<0.05 ;+, P<0.10$. 

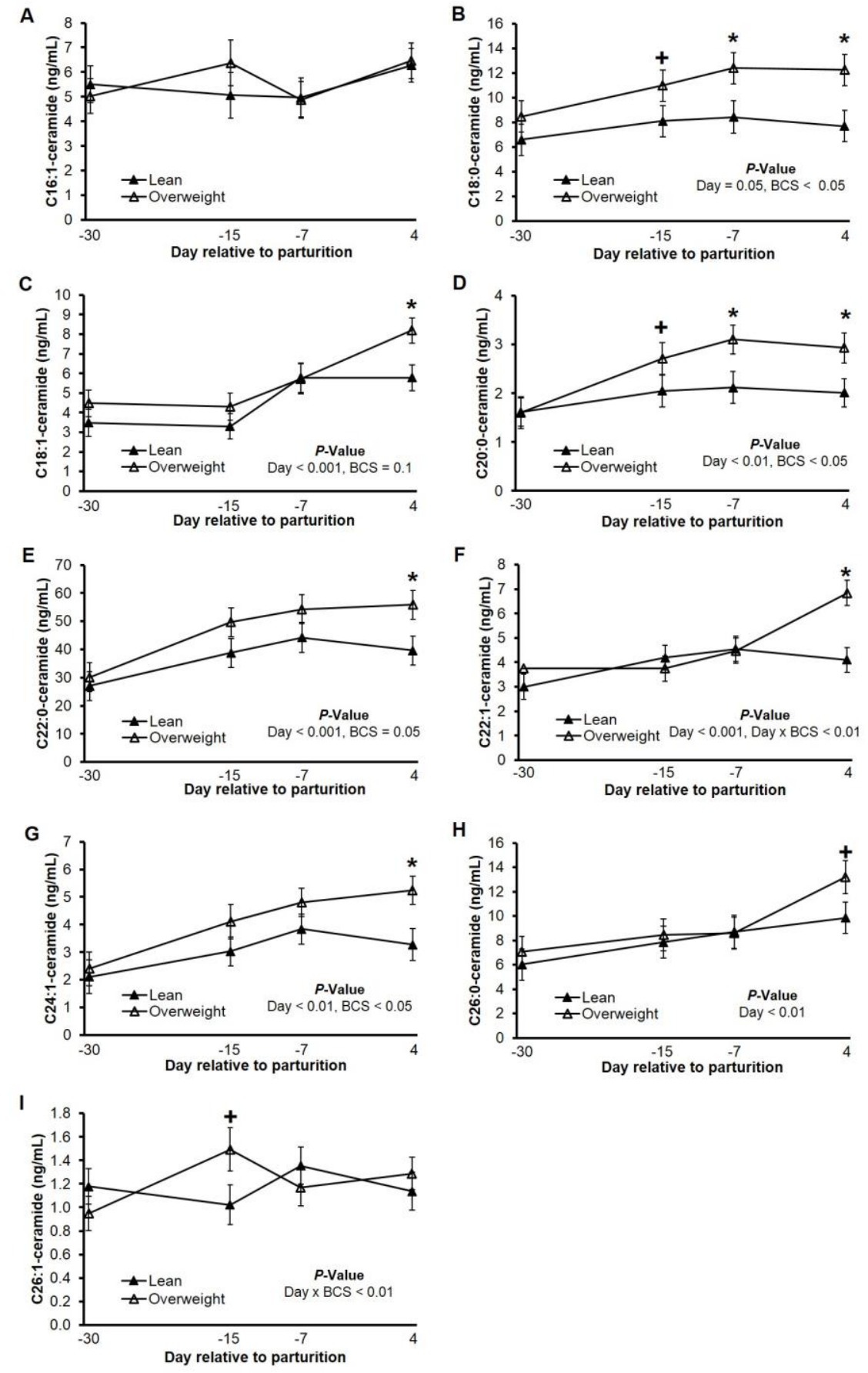

Figure 3-10. Supplemental 3-1. Plasma concentrations (ng/mL) of low-abundant ceramides in lean and overweight cows transitioning from late pregnancy to early lactation, relative to lean cows.

Plasma concentrations of (A) C16:1-, (B) C18:0-, (C) C18:1-, (D) C20:0-, (E) C22:0-, (F) C22:1-, (G) C24:1, (H) C26:0-, and (I) C26:1-ceramide in lean and overweight transition dairy cows. Data are represented as least squares means and their standard errors. ${ }^{*}, P<0.05 ;+, P<$ 0.10 . 

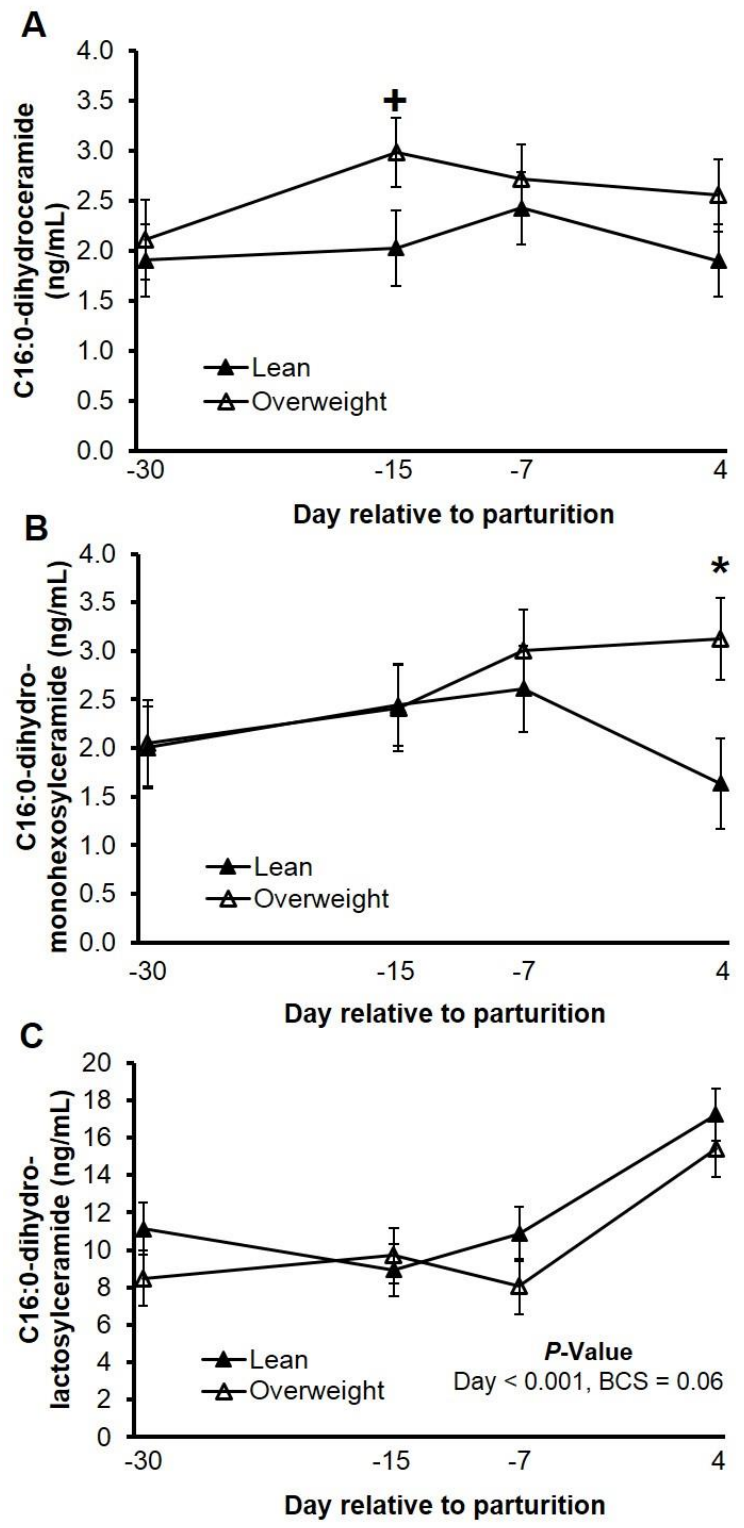

Figure 3-11. Supplemental 3-2. Plasma concentrations (ng/mL) of C16:0-linked dihydroceramide, dihydro-monohexosylceramide, and dihydro-lactosylceramide in lean and overweight cows transitioning from late pregnancy to early lactation, relative to lean cows.

Plasma concentrations of C16:0-linked (A) dihydroceramide, (B) dihydro-monohexosylceramide, and $(\mathbf{C})$ dihydro-lactosylceramide in lean and overweight transition dairy cows. Data are represented as least squares means and their standard errors. ${ }^{*}, P<0.05 ;+, P<0.10$. 


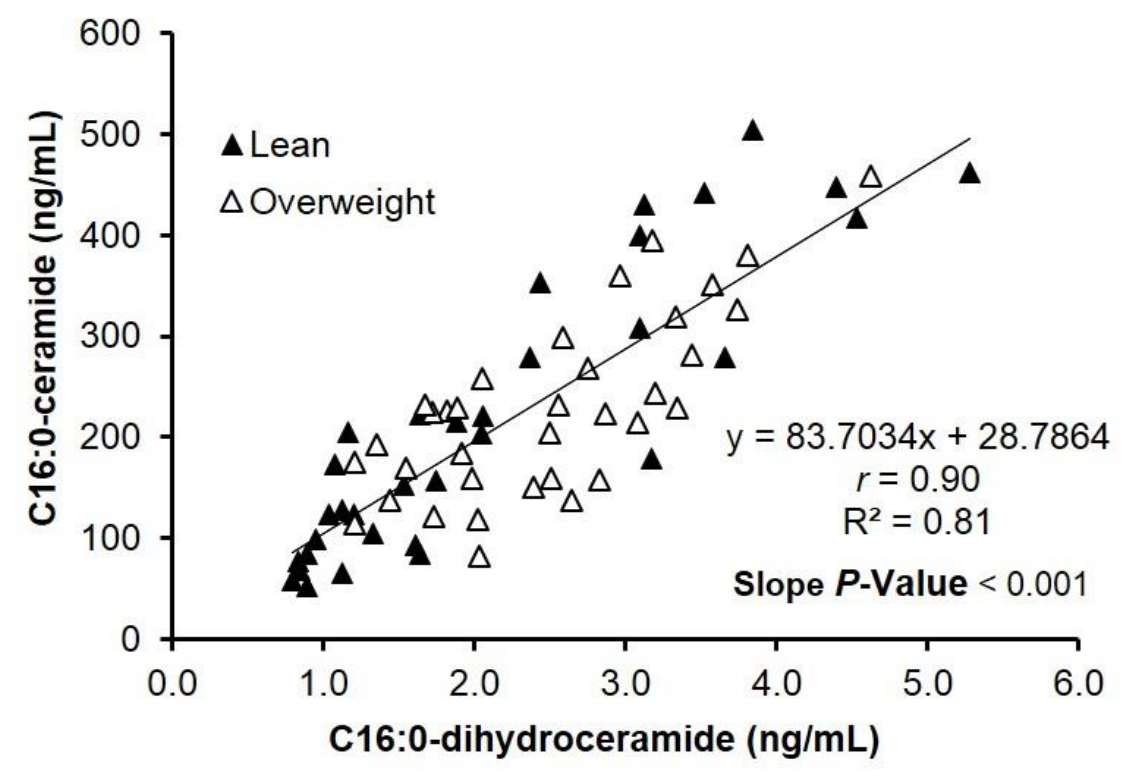

Figure 3-12. Supplemental 3-3. Plasma C16:0-dihydroceramide is positively correlated with C16:0-ceramide.

Simple linear regression analysis between plasma concentrations of C16:0-dihydroceramide and C16:0-ceramide in lean and overweight dairy cows during the transition from late pregnancy to early lactation. Data represents measurements for d $-30,-15,-7$, and 4 , relative to parturition. 

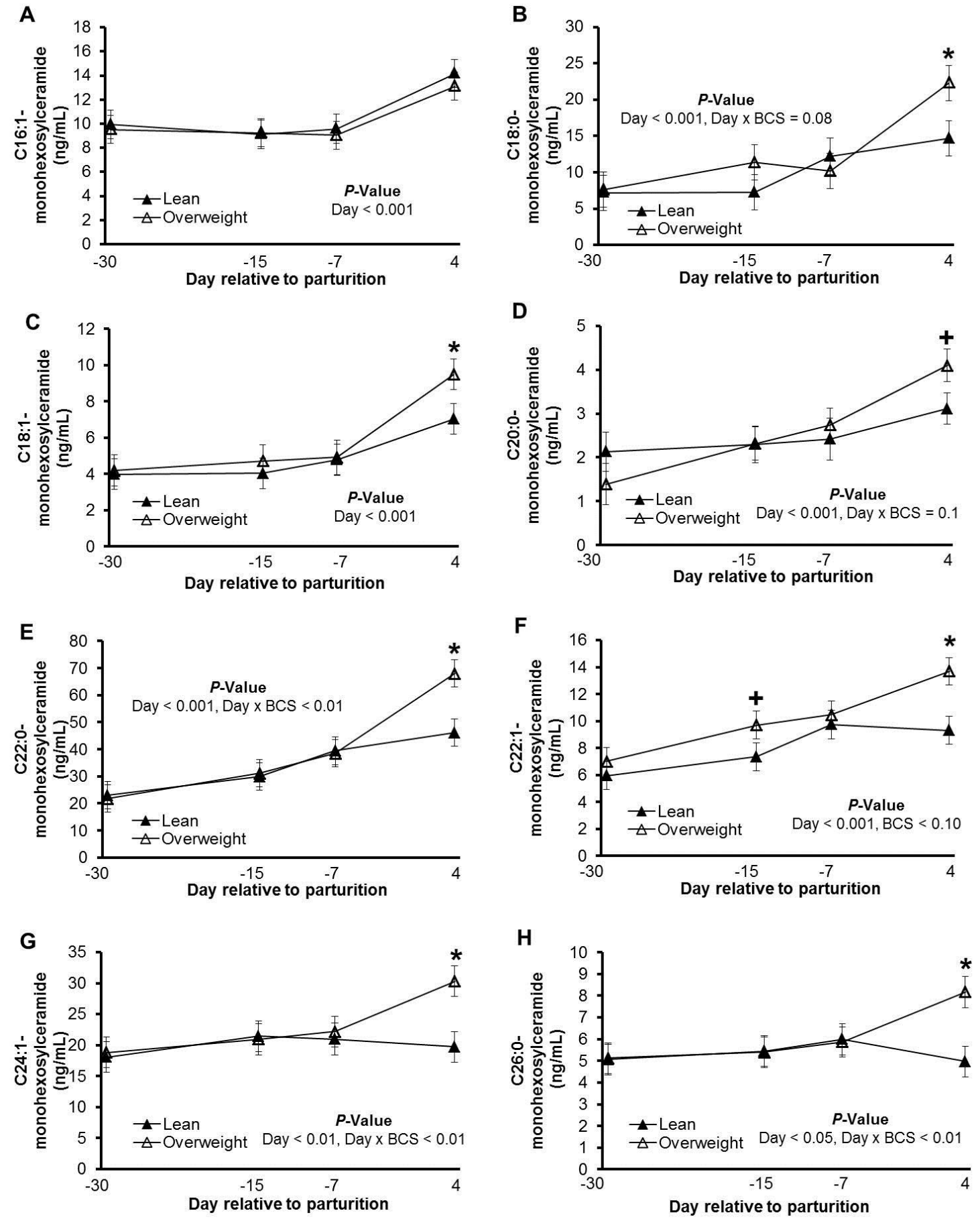

Figure 3-13. Supplemental 3-4. Plasma concentrations (ng/mL) of low-abundant monohexosylceramides in lean and overweight cows transitioning from late pregnancy to early lactation, relative to lean cows.

Plasma concentrations of (A) C16:1-, (B) C18:0-, (C) C18:1-, (D) C20:0-, (E) C22:0-, (F) C22:1, (G) C24:1, and (H) C26:0-monohexosylceramide in lean and overweight transition dairy cows. Data are represented as least squares means and their standard errors. ${ }^{*}, P<0.05 ;+, P<0.10$. 

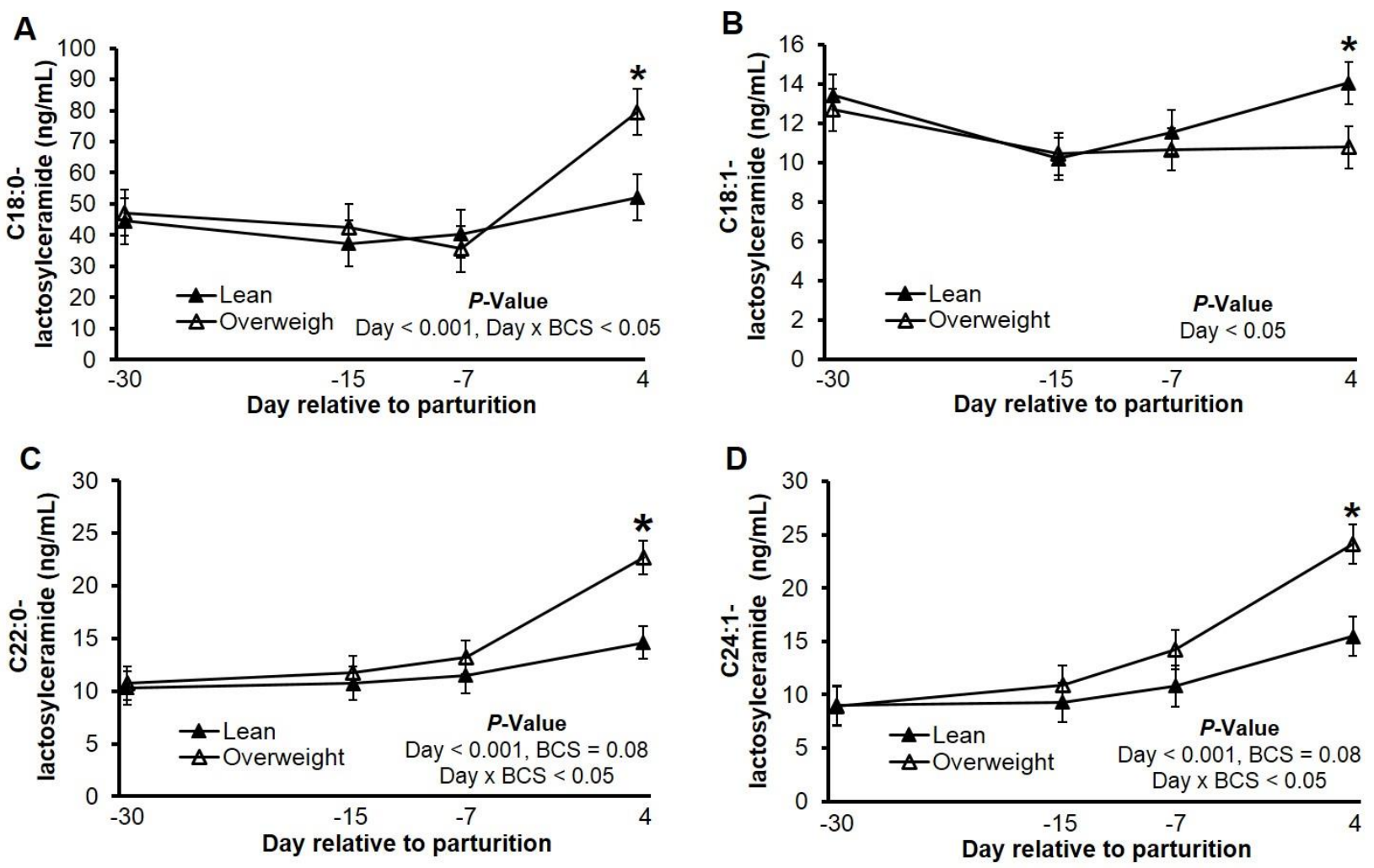

Figure 3-14. Supplemental 3-5. Plasma concentrations (ng/mL) of low-abundant lactosylceramides in lean and overweight cows transitioning from late pregnancy to early lactation, relative to lean cows.

Plasma concentrations of (A) C18:0-, (B) C18:1-, (C) C22:0-, and (D) C24:1-lactosylceramide in lean and overweight transition dairy cows. Data are represented as least squares means and their standard errors. *, $P<0.05 ;+, P<0.10$. 


\title{
Chapter 4
}

\section{TEMPORAL CHANGES IN CIRCULATING AND TISSUE SPHINGOLIPIDS DURING THE TRANSITION FROM GESTATION TO LACTATION.}

\begin{abstract}
Insulin is fundamentally involved in the homeorhetic adaptations that accompany late gestation and early lactation in dairy cows. Insulin resistance develops naturally during the peripartal period and is associated with increased lipolysis in adipose tissue, as well as hepatic fat accumulation. Increased fatty acid availability favors accumulation of the sphingolipid ceramide in monogastrics and in peripartal dairy cows. Ceramide is associated with the pathogenesis of insulin resistance in obese models of type 2 diabetes and humans with metabolic disease. In addition, limited evidence suggests that ceramides may be involved in processes that facilitate labor in humans, thus supporting a physiological roles for ceramide during perpartum. Despite this evidence, the relationship between sphingolipids and insulin action during the transition from gestation to lactation is currently unknown. Therefore, our objectives were to characterize temporal responses in plasma and tissue sphingolipids during peripartum, and to determine whether these changes were related to adiposity, lipolysis, and insulin action. Multiparous Holstein cows were grouped by adiposity score (BCS) at d-28 prepartum as either lean (BCS $2.9 \pm 0.13$; $n$ $=7$ ) or overweight (BCS $4.0 \pm 0.21 ; n=7$ ). Blood samples were collected routinely from d-21 to 21, relative to calving, and liver and skeletal muscle biopsies were obtained at d-21, -7 , and 4, relative to parturition. Liquid chromatography and tandem mass spectrometry was utilized to profile ceramides, monohexosylceramides (GlcCer), lactosylceramides (LacCer), and sphingomyelins with variable acyl-chain lengths and degree of saturation. Plasma C16:0-ceramide
\end{abstract}


concentration was higher in overweight cows prepartum and decreased significantly during postpartum, in association with increased C16:0-GlcCer. C24:0-ceramide, the most abundant ceramide in plasma, increased by $50 \%$ across the transition period and was higher in overweight during postpartum. Moreover, C24:0-ceramide in plasma was positively associated with hepatic fat accumulation. Glycosylated ceramides in plasma increased postpartum, but were not affected by adiposity. Plasma sphingomyelin, a source of ceramides, decreased toward parturition and increased postpartum. Sphingomyelins were lower in the plasma of overweight cows during the peripartal period. Hepatic C24:0-Cer was the most abundant ceramide in the liver, and its concentration increased postpartum and was positively associated with plasma C24:0 ceramides. Several hepatic ceramides and glycosylated ceramides were higher in overweight cows during postpartum. Muscle ceramide, on the other hand, was higher in lean cows prepartum, and increased in overweight cows during the transition from gestation to lactation. Plasma ceramides were positively associated with plasma NEFA and inversely associated with glucose disappearance during an insulin challenge. Moreover, glycosylated ceramides were inversely associated with reduced insulin secretion. Our results demonstrate that plasma and tissue sphingolipids change dynamically during the transition from gestation to lactation, and are associated with the onset of peripartal insulin resistance. These observations are in line with a possible role for certain ceramides in facilitating the physiological adaptations of peripartum. Future research should focus on directly evaluating these possibilities. 


\section{Introduction}

Major physiological adaptations accompany late pregnancy and early lactation in dairy cows, which allow uninterrupted nutrient supply to the fetus (prenatally) and to the newborn calf (postnatally). During the last trimester of lactation, the growth of the conceptus (fetus and fetal membranes) follows an exponential curve, driving increased demands for energy (glucose) and protein for the gravid uterus (Eley et al., 1978, Bell et al., 1995; NRC, 2001). Deposition of fat, protein, and glycogen account for most of the energy content of fetal tissues during late pregnancy (Bell et al., 1995). Once lactation is initiated, the synthesis of milk and its components typically results in a doubling of daily energy requirements in modern, high-producing dairy cows (Drackley et al., 2005). Moreover, increased glucose flux toward the mammary gland favors lactose synthesis and results in a 3-4 fold increased in daily glucose requirements (Bell, 1997). In order to cope with these localized nutrient demands, tight regulation of metabolism is required during the transition from gestation to lactation. The control of metabolism during this period involves homeostatic as well as homeorhetic regulation (Bauman and Currie, 1980). In particular, homeorhetic control allows coordinated shifts in metabolism in tissues such as the adipose tissue, skeletal muscle, liver, and the mammary gland. Metabolic changes that facilitate increased glucose partitioning toward the fetus and the mammary gland include a progressive reduction of insulin secretion and responsiveness, (Bell, 1995; Bell and Bauman, 1997), as well as circulating endocrine regulators, such as growth hormone, estrogens, and progesterone, among others (Tucker, 1985). As a result, dairy cows experience increased lipolysis (McNamara, 1991), hepatic ketogenesis and gluconeogenesis, thus increasing glucose flux into the mammary gland (Bell, 1995, Spachmann et al., 2013). 
The development of insulin resistance in associated with the accumulation of ceramide in models of obesity and insulin resistance (Summers et al., 1998; Haus et al., 2009). Ceramides are anti-anabolic sphingolipids with key regulatory functions such as control of cell cycles, cell death, and response to stress (Hannun and Obeid, 2008). Although they are diverse chemical entities, all ceramides are composed of a sphingoid backbone which is acylated with many possible fatty acid residues (Gault et al., 2010; Chen et al. 2010) differing in chain length and degree of saturation, by the activity of several ceramide synthases (i.e. C2-C26; Levy and Futerman, 2010). Two major pathways contributing to ceramide accumulation in cells are de novo ceramide synthesis and sphingomyelin hydrolysis (Figure 5-1). De novo ceramide synthesis uses palmitoyl CoA and serine to produce ceramides (Holland and Summers, 2008), and the flux through this pathway is strongly influenced by saturated fatty acid (SFA) availability and inflammatory signals, such as TNF $\alpha$ and IL-6 (Merrill, 2002; Samad et al., 2006; Senn, 2006). Sphingomyelins are highly abundant sphingolipids in plasma membranes, and consist of a phosphorylcholine ester-bound to ceramide. The hydrolysis of sphingomyelin can generate ceramide in a reaction catalyzed by sphingomyelinase (SMase) enzymes, which differ in their optimal $\mathrm{pH}$ and cellular locations (Deigner et al., 2009; Boulgaropoulos, et al., 2010; Figure 5-1). This pathway is commonly upregulated in obesity via the activation of inflammatory signals, which promote increased levels of SMase (Samad et al., 2006).

Ceramides are commonly associated with excessive body fat and human type 2 diabetes, and constitute a key causative link between circulating SFA availability and insulin resistance (Chavez et al., 2003). Moreover, ceramides accumulate in insulin resistant tissues of rodents (Summers, 2006; Holland and Summers 2008) and humans (Adams et al., 2004; Straczkowski, 2007). Using a dairy cow model, we have recently demonstrated that ceramides accumulate during 
late pregnancy and the first week of lactation in lean and overweight dairy cows (Rico et al., 2015). Moreover, changes in plasma ceramide were associated with circulating NEFA availability, suggesting that ceramide synthesis through de novo pathway may occur during peripartum, particularly in overweight cows experiencing increased lipolysis. Along the same lines, because the peripartal period is characterized by a state of inflammation (Sordillo and Raphael, 2013), ceramide supply from sphingomyelin could be upregulated during this period. Although many cell types have the capacity synthesize ceramides (Levy and Futerman, 2010), the liver is considered to be the primary source of circulating ceramides (Watt et al., 2012). This supported by the observation that the vast majority of plasma ceramides are found in association with the liverderived low- and very low-density lipoproteins (LDL and VLDL, respectively), fractions in which C24:0-ceramide represents the major ceramide (Weisner et al., 2009). In view of these observations, and considering that the peripartal increase in NEFA typically results in hepatic fat accumulation in dairy cows, we hypothesized that hepatic ceramide content would be elevated during the transition from gestation to lactation. Similarly, considering that the skeletal muscle is responsible for most of the insulin-stimulated whole body glucose uptake in dairy cows (Duhlmeier et al., 2015), and given that ceramide accumulation is commonly observed in skeletal muscle of models of insulin resistance and obesity (Lipina and Hundal, 2011; DeFronzo, 2004; Boon et al., 2013), we were interested in determining ceramide accumulation in skeletal muscle of periparturient dairy cows. We further hypothesized that overweight peripartal cows would exhibit greater concentrations of ceramide in plasma, liver and muscle, and that ceramide accumulation would be associated with insulin resistance. To test our hypotheses, we used a lipidomics approach, which employed liquid chromatography coupled with mass spectrometry, 
and characterized ceramides, glycosylated ceramides, and sphingomyelins in dairy cows transitioning from gestation to lactation.

\section{Materials and Methods}

\section{Experimental Design}

Experimental procedures were approved by the West Virginia University Institutional Animal Care and Use Committee (Morgantown). The experiment was completed at Dovan Farms, WVU Agricultural Research and Education Partner, and a 700-Holstein cow commercial dairy farm (located in Berlin, PA). Nonlactating, pregnant, multiparous Holstein cows $(\mathrm{n}=14)$ were enrolled in the study $30 \mathrm{~d}$ before expected parturition and allocated to 1 of 2 groups according to their adiposity, as either lean (BCS $2.91 \pm 0.13, \mathrm{n}=7$ ) or overweight (BCS $4.0 \pm 0.21, \mathrm{n}=7$ ). Cows were housed in a free-stall barn and trained to access feed by using Calan gate feeders (American Calan, Inc., Norwood, NH). Diets were formulated to meet nutrient recommendations and cows were fed once and twice daily during pregnancy and lactation, respectively. Cows were fed at $110 \%$ of expected intake, adjusted daily, and provided free access to water. Ingredient and nutrient compositions of the diets fed during the experiment are shown in Table 4-1. Samples of TMR were obtained weekly throughout the experiment, and DM content was determined by drying at $60^{\circ} \mathrm{C}$ until a static weight was observed. Body weights and BCS were recorded weekly. Three trained investigators independently recorded BCS for all cows and data were averaged for each cow at d $-30-21,-14,-7$, relative to expected parturition, and d $0,7,14$, and 21 postpartum, using 
a 1.0 to 5.0 scale (Wildman et al., 1982). Blood samples $(10 \mathrm{~mL}$ ) were collected by coccygeal venipuncture at, $-30,-21,-7$, and -5 , relative to expected parturition, and $0,2,4,7,14$, and 21 postpartum. Blood was kept on ice for $30 \mathrm{~min}$ until centrifugation at $3,400 \times \mathrm{g}$ for $10 \mathrm{~min}$. Following centrifugation, plasma was removed and snap-frozen in liquid nitrogen and then stored at $-80^{\circ} \mathrm{C}$ until further analysis. Milk production was recorded from d 2 to 21 postpartum. Milk samples were collected at d 2, 3, 4, 5, 6 and 7 postpartum, preserved using Bronopol tablets (D\&F Control Systems, San Ramon, CA), and stored at $4^{\circ} \mathrm{C}$ for milk component analysis.

\section{Tissue Biopsies}

Liver, and skeletal muscle biopsies were performed on $\mathrm{d}-21,-7$ and 4 relative to the parturition using aseptic techniques. For tissue biopsies, cows were anesthetized with xylazine $(0.04 \mathrm{~mL} / \mathrm{kg}$ of BW) delivered via the coccygeal vein. After the hair was clipped, biopsy sites were sanitized with iodine scrub and anesthetized with a $5 \mathrm{~mL}$ of lidocaine $\mathrm{HCl}$ (Vedco Inc., Saint Joseph, MO) delivered subcutaneously. For liver biopsies, the $11^{\text {th }}$ intercostal space was prepared by making a $0.5 \mathrm{~cm}$ incision through the skin, and a fabricated trocar was utilized to collect approximately $1 \mathrm{~g}$ of liver tissue (Hughes, 1962). Skeletal muscle biopsies were performed by making a $0.5 \mathrm{~cm}$ incision in the skin adjacent to the semitendinosus muscle. Approximately 500 mg of muscle was collected using a Bard Magnum biopsy instrument mounted with a 12 gauge needle (Bard Biopsy Systems, Tempe, AZ). Following the collection of tissue, biopsy sites were stapled and sprayed with antiseptic, and Excenel RTU was delivered once by intramuscular injection $(0.68 \mathrm{~mL} / \mathrm{kg}$ of BW; Zoetis, Florham Park, NJ). Tissue for ceramide profiling was snapfrozen in liquid nitrogen and stored at $-80^{\circ} \mathrm{C}$. Tissue for PCR analysis was stabilized using 
RNAlater (Thermo Fisher Scientific Inc, Waltham, MA), refrigerated at $4^{\circ} \mathrm{C}$ during $24 \mathrm{~h}$, and stored at $-20^{\circ} \mathrm{C}$ until RNA isolation was performed.

\section{Insulin Tolerance Tests}

Insulin tolerance tests (ITT) were performed on $\mathrm{d}-20,-6$ and 5 , relative to parturition using previously described methods (Pires et al., 2007a). Briefly, a jugular catheter was inserted $24 \mathrm{~h}$ prior to the ITT, and patency was maintained by flushing with heparinized saline every $12 \mathrm{~h}$. Access to feed was blocked $2 \mathrm{~h}$ prior to ITT start. Cows were intravenously infused with 0.1IU of insulin (100 IU/mL; human insulin rDNA origin; Eli Lilly Co., Indianapolis, IN) per kg of BW, and followed by a 10-mL saline solution flush. Blood samples $(10 \mathrm{~mL})$ were collected at $-10,0$, $10,20,30,40,60,90,120,150$, and 180 min relative to insulin infusion. Blood was processed as described above. Feed was provided immediately following the completion of the ITT. The area under the curve for glucose during ITT was calculated using the trapezoidal method as previously described by others (Pires et al., 2007a).

\section{Sample Analyses}

Individually composited feed ingredients were analyzed for NDF with heat-stable $\alpha$ amylase and sodium sulfite (Van Soest et al., 1991), CP (AOAC International, 2000; method 990.03), and starch (Hall, 2009) by Cumberland Valley Analytical Services Inc. (Cumberland, MD).

Plasma samples were analyzed for glucose, insulin, NEFA, BHBA, and ceramides. Plasma concentrations of glucose, NEFA, and BHBA were determined by enzymatic methods using 
commercial kits (Autokit Glucose, HR Series NEFA-HR (2) and Autokit 3-HB, respectively; Wako Chemicals USA Inc., Richmond, VA). Plasma concentrations of insulin were determined by ELISA using a commercial product (Mercodia Bovine Insulin ELISA; Mercodia AB, Uppsala, Sweden). All spectrophotometric measurements were conducted using a SpectraMax Plus 384 Microplate Reader (Molecular Devices, Sunnyvale, CA). Individual milk samples were analyzed for fat, true protein, and lactose concentrations by mid-infrared spectroscopy (Dairy One, Ithaca, NY; AOAC, 1990; method 972.160) within 1 wk of collection. Hepatic liver lipid was extracted and quantified using the methodology described by Starke and collaborators (2010).

Ceramide extraction was conducted using previously established methods that employ a modified Bligh and Dyer procedure including ceramide C12:0 as an internal standard (Avanti Polar Lipids, Alabaster, AL; Haughey, et al., 2004; Bandaru et al., 2013; Rico et al., 2015). Briefly, extracts were dried using a nitrogen evaporator and re-suspended in pure methanol for analysis. Sample extracts were handled using an autosampler (LEAP technologies Inc., Carrboro, NC) that introduced extracts into an HPLC (PerkinElmer, Boston, MA) with a C18 reverse-phase column (Phenomenex, Torrance, CA). Individual species of ceramide were separated by gradient elution and injected into an electrospray ion source coupled to a triple quadrupole mass spectrometer (API3000; AB Sciex Inc., Thornhill, Ontario, Canada; (Bandaru et al., 2007; Bandaru et al., 2011; Bandaru, 2013). Ion spray voltage was $5500 \mathrm{~V}$ at a temperature of $80^{\circ} \mathrm{C}$ with a nebulizer gas of 8 psi, curtain gas 8 psi, and collision gas 4 psi. Declustering potential was $80 \mathrm{~V}$, focusing potential $400 \mathrm{~V}$, entrance potential $10 \mathrm{~V}$, collision energy $30 \mathrm{~V}$, and collision cell exit potential $18 \mathrm{~V}$. Individual ceramide species were detected by multiple reaction monitoring. Six point calibration curves $(0.1$ to $750 \mathrm{ng} / \mathrm{ml})$ were constructed by plotting area under the curve for each calibration standard, C16:0-, C18:0-, C20:0-, C22:0-, and C24:0-ceramide, normalized to C12:0-ceramide. 
Sphingolipid concentrations were determined by curve fitting the identified ceramide species based on acyl-chain length. Quantification of spectral data was performed using Analyst 1.4.2 and MultiQuant software (AB Sciex Inc., Thornhill, Ontario, Canada).

\section{Calculations and Statistical Analysis}

Plasma glucose, insulin, NEFA, and BHBA concentrations were measured. Changes in BCS and BW of lean and overweight cows were calculated as the differences between values at $\mathrm{d}$ -21 and 21, relative to parturition, and were analyzed using the GLM procedure of SAS (version 9.3; SAS Institute Inc., Cary, NC). All data for time-dependent changes in plasma, tissue and milk variables were analyzed as repeated measures over time relative to parturition under the MIXED procedure of SAS (SAS Institute Inc.). The statistical model included the random effect of cow nested within BCS and the fixed effects of BCS, day relative to parturition, and their interaction. The covariance structure was modeled to identify patterns that best describe relationships between the repeated measures. The most appropriate covariance structure was selected for each response variable after evaluating 3 different covariance structures (variance components, first-order autoregressive, heterogeneous first-order autoregressive, compound symmetry), and the structure with the smallest Akaike's information criterion coefficient was selected for analysis. The method of Kenward-Roger was used for calculation of denominator degrees of freedom. Preplanned contrasts were used to evaluate differences between lean and overweight cows at each time point. Relationships between select ceramides were evaluated by random regression, using the MIXED procedure of SAS (SAS Institute Inc.). The full model included the random effect of cow and the linear and quadratic effects of each predictor. Quadratic effects were removed from the model 
based on fit by Akaike's information criterion scores when $P>0.05$. Denominator degrees of freedom were calculated by the Satterthwaite method. Studentized residual values $>3.0$ or $<-3.0$ were considered outliers and removed from the analysis (typically 1 per response variable). Parametric pearson correlations were performed to determine associations between plasma sphingolipids, NEFA levels, and glucose disposal, and between plasma and hepatic ceramides. All results are expressed as least squares means and their standard errors, unless stated otherwise. Significance was declared at $P<0.05$ and tendencies at $P<0.10$.

\section{Results and Discussion}

Ceramides of hepatic origin have the ability to disrupt insulin signaling, and accumulate in plasma from type 2 diabetic and obesity animal models, as well as in overweight dairy cows transitioning from gestation to lactation (Haus et al., 2009; Watt et al., 2012; Rico et al., 2015). Moreover, ceramides accumulate around parturition in healthy humans and healthy dairy cows (Signorelli et al., 2016; Rico et al., 2015), suggesting they may fulfil metabolic roles independently of body fat accumulation. Two major pathways contributing to ceramide accumulation include $d e$ novo synthesis and sphingomyelin hydrolysis, processes upregulated in association with increased NEFA availability and peripartal inflammation (Watt et al., 2012; Peraldi et al., 1996; Signorelli et al., 2016; Figure 4-1). Therefore, we evaluated the temporal changes in sphingolipids in plasma, liver and skeletal muscle in lean and overweight cows transitioning from gestation to lactation $(\mathrm{d}$ -21 to 21 relative to parturition). Because adipose tissue lipolysis increases during peripartum, and can contribute to ceramide synthesis and insulin resistance, we evaluated the associations between circulating NEFA, sphingolipids and insulin action. In order to measure changes in circulating and 
tissue ceramide levels, we employed liquid chromatography coupled with mass spectrometry to perform a sphingolipidomic analysis of plasma ceramides, glycosylated ceramides, and sphingomyelins This approach, described by Merrill et al. (2009), was utilized previously by our research group (Rico et al., 2015), and allowed us to quantify 37 different ceramides, GlcCer, LacCer, and sphingomyelins with differing acyl chain length and degree of saturation in plasma, liver and skeletal muscle.

Detailed production and metabolic responses will be described briefly (unpublished data). Compared with lean, overweight cows exhibited decreased appetite, increased body weight loss and body fat loss, and had elevated fat accumulation in the liver during the peripartal period. Although no differences in milk yield were detected, overweight cows had increased milk fat content and secretion, a consequence of increased lipomobilization. Overweight cows displayed increased plasma NEFA and BHBA during peripartum, and were hyperinsulinemic relative to lean cows prepartum. Insulin concentration decreased gradually as parturition approached, and the rate of glucose disposal in response to insulin challenge decreased postpartum, indicating reduced insulin sensitivity after parturition.

Dynamic changes were observed in the concentrations of circulating ceramides and glycosylated ceramides during peripartum (Figure 4-2; Figure 4-3). Most notably, glycosylated ceramides increased around parturition, and were 35\% and 53\% higher in average for GlcCer and LacCer during postpartum $(P<0.001)$. As expected, NEFA correlated positively with ceramides, GlcCer and LacCer, $(r=0.22,0.5$ and 0.49 , respectively; $P<0.05)$, consistent with an increase in substrate flux in the de novo ceramide synthesis pathway. Although no temporal changes were observed for total ceramide, individually, plasma ceramides displayed different patterns of change across time during transition (Figure 4-4). For example, C16:0-ceramide, one the major ceramides 
found in plasma (Rico et al., 2016), decreased sharply by d 2 , relative to $\mathrm{d} 0(-32 \%, P<0.05)$, and remained low during postpartum. In contrast, C18:0-ceramide increased acutely around parturition, peaking at d $0(+40 \%$, relative to prepartum), and decreased gradually over the $3 \mathrm{wk}$ period postpartum. The diversity of patterns in the changes of plasma ceramide, in addition to the stability in the predominant C24:0-ceramide, explain the lack of time associated effects for total ceramides, and suggest that peripartal ceramide metabolism is acyl-chain specific. In validation of our previous investigation of ceramide metabolism during peripartum (Rico et al., 2015), several plasma ceramides were elevated in overweight cows pre- and postpartum (i.e. C16:0-, C18:0-, C22:0-, C24:0- and C26:0-ceramide; Figure 4-4).

Following a similar trend to that of total GlcCer, most plasma GlcCer increased around parturition and remained elevated postpartum (C16:0-, C18:1-, C20:0-, C22:0-, and C24:0; $P<$ 0.001, Figure 4-5). Overall, plasma GlcCer tended to be elevated in overweight cows, and were significantly higher postpartum for some low-abundant GlcCer (e.g. 16:1- and C22:0-GlcCer). Interestingly, an inverse relationship between C16:0-GlcCer and C16:0-ceramide was observed $(r$ $=0.4 ; P<0.001)$. In our previous examination of plasma ceramides (Rico et al., 2015), C16:0ceramide was the only major ceramide to decrease postpartum, and observation validated by our current data. Because glucosyceramide synthase is responsible for ceramide glycosylation (Bleicher and Cabot, 2002), glucosylceramide can modulate ceramide metabolism and reduce its availability for use in other cellular processes (Liu et al., 2013). Although acyl chain specificity has not been demonstrated for glucosylceramide synthase, a preference for the highly abundant C16:0-ceramide could significantly affect the plasma ceramide pool. From these observations, it is possible that active glycosylation of C16:0-ceramide is responsible for a reduction in its 
availability during postpartum. Similarly, increased glycosylation of other ceramides could explain their accumulation around parturition and during postpartum.

Plasma LacCer were consistently elevated postpartum $(P<0.01$; Figure 4-6). For example, C16:0-LacCer, the major plasma lactosylceramide, was $44 \%$ higher during postpartum, and remained elevated during this period $(P<0.001)$. In addition, most plasma LacCer detected were higher in overweight cows during postpartum (i.e. C18:0, C22:0-, C24:0-, C24:1-lacCer; $P<0.01$ ). Because the effects of LacCer on insulin signaling have yet not been established, the significance of these changes cannot be asserted. However, it should be noted that LacCer represented 53\% of the total plasma ceramides, followed by simple ceramides (26\%) and Glccer (20.8\%). Because lactosylceramides are precursors of gangliosides GM3 (Chavez et al., 2014), their increased accumulation has the potential to impact cellular metabolism.

Similar to the profile of circulating sphingomyelins in adult humans (Hanamatsu et al., 2014), C16:0-sphingomyelin was the predominant plasma sphingomyelin found in the peripartal period, representing nearly $35 \%$ of total circulating sphingomyelin, and followed by C18:1-, C20:1-, C24:1, and saturated-chain sphingomyelin (Figure 4-7). Total plasma sphingomyelin decreased gradually toward parturition and was $30 \%$ lower at $\mathrm{d} 0$, relative to $\mathrm{d}-21(P<0.001)$. Similarly, the concentration of all circulating sphingomyelins and dihydro-sphingomyelins decreased toward parturition and recovered to initial levels by wk 3 postpartum (Figure 4-8; Figure 4-9; Figure 4-15). This consistent pattern of change is suggestive of extensive sphingomyelin degradation, which reaches a maximum at parturition. In addition, several plasma sphingomyelin were consistently lower in overweight cows during the transition from gestation to lactation (e.g. C16:1-, and the abundant C18:1- and C20:1-sphingomyelin), indicating that sphingomyelin hydrolysis can contribute to ceramide accumulation during the entirety of the transition in 
overweight cows. Because ceramides can be produced by sphingomyelin degradation, we hypothesize that a mechanism to facilitate ceramide accrual at parturition is responsible for the observed reduction in sphingomyelin concentrations. This would be consistent with the recent observation by Signorelli and collaborators (2015) that ceramide accumulates in placental tissue of humans at parturition, driving inflammatory responses to reduce the duration of labor. Inflammation is also a common physiological response observed in dairy cows close to calving (Sordillo and Raphael, 2013), and it is known to drive sphingomyelin hydrolysis through the action of inflammatory cytokines (Yang et al., 1993; Kolesnick and Golde, 1994; Peraldi et al., 1996). Although it is possible that ceramide accumulation in tissues serves a similar purpose in periparturent dairy cows, it could also facilitate other physiological responses around parturition as it accumulates in different tissues. Although the overall relationship between plasma ceramides and sphingomyelin was positive, the correlation coefficient was moderately low $(r=0.25 ; P<$ 0.05), the association between individual plasma ceramides and sphingomyelin was equivocal. While the major plasma ceramide, C24:0, was positively associated with total sphingomyelin $(r=$ 0.34; $P<0.01$ ), other ceramides were inversely related to sphingomyelin. For example, plasma C18:0-ceramide concentration was negatively associated with sphingomyelin, and had the strongest correlation coefficient of all plasma ceramides $(r=-0.6 ; P<0.001)$. Moreover, changes in C18:0- and C18:1-ceramide showed a moderately high association with C18:0-sphingomyelin $(r=-0.47$ and -0.58 , respectively; $P<0.001)$, indicating a substrate to product relationship within acyl chain length. This is supported by the observation that the peripartal increase in C18:0ceramide was mirrored by an inverse decrease in the concentration of plasma sphignomyelin (Figure 4-4 and Figure 4-9). The potential importance of the sphingomyelin hydrolysis pathway to provide ceramide is highlighted by the observation that sphingomyelin comprises the great 
majority of the sphingolipids we measured in plasma. On average, plasma sphingomyelin was 2600-fold higher than plasma ceramides and 680-fold higher than all ceramides and glycosylated ceramides combined. This suggest that even small changes in the concentration of sphingomyelin could greatly affect ceramide accumulation.

Because the development of insulin resistance is associated with the accumulation of ceramides in insulin tissues such as liver and skeletal muscle, and results in the disruption of insulin signal transduction associated with the pathogensis of type 2 diabetes (Lipina and Hundal, 2011; DeFronzo, 2004; Boon et al., 2013), we measured the ceramide content of liver and skeletal muscle. The liver is considered the main source of plasma ceramides, as evidenced by the fact that $75 \%$ to $98 \%$ of total plasma ceramides are found contained within the apoB-containing low-, and very low-density lipoproteins (LDL and VLDL; Weisner et al., 2009; Iqbal et al., 2015), as well as in high density lipoproteins (HDL; Lightle et al., 2003; Boon et al., 2013). Similar to the profile of plasma ceramides we have previously reported in peripartal dairy cows (Rico et al., 2015), we observed that C24:0-ceramide and C24:0-Glccer and C16:0-Laccer were the major ceramides found in hepatic tissue and skeletal muscle of hyperlipidemic, insulin resistant cows at d4 posptpartum (Figure 4-10; Figure 4-14). Specifically, C24:0-ceramide represented 70\% and 65\% of total ceramides in liver and skeletal muscle, respectively. The importance of C24:0 ceramide accumulation is highlighted by the findings of Boon and collaborators (2013), who showed that infusing LDL-bound C24:0-ceramide to mice resulted in whole body and skeletal muscle insulin resistance (Boon et al., 2013). Time-related changes in hepatic ceramides and glycosylated ceramides of lean and overweight cows are shown in Figure 4-11 and Figure 4-16. Unexpectedly, hepatic C24:0-ceramide content tended to be lower, while total ceramide was significantly lower in overweight cows at d -24 prepartum. However, both C24:0-ceramide and total ceramide 
increased toward parturition in overweight cows only $(\mathrm{BCS} \times$ Day $=0.05$; Figure 4-11) and were $68 \%$ and $62 \%$ higher by d 4 postpartum, respectively $(P<0.001)$. Moreover, regression analysis revealed a positive linear relationship between liver and plasma ceramides (Figure 4-13; $P<$ 0.001). Plasma C24:0- and total ceramide showed moderately high associations with hepatic C24:0- and total ceramide $(r=0.54$ and $0.63 ; P<0.05)$. These results are in agreement with the role of the liver as a source of circulating ceramides (Watt et al., 2012; Weisner et al., 2009), which can reach organs such as the skeletal muscle and induce insulin resistance.

In the current study, hepatic fat accumulation was greater in overweight cows and it was associated with increased plasma NEFA around parturition (unpublished data), indicating accumulation of fatty acid substrates for ceramide synthesis. Along these lines, in a recent study, Xia and collaborators (2015) demonstrated that ceramide accumulation can contribute to hepatic steatosis and insulin resistance in mice, by promoting $\mathrm{PKC} \zeta$ activation and $\mathrm{CD} 36$-mediated lipid uptake in the liver. In dairy cows, excessive lipomobilization results in elevated NEFA and is associated with clinical fatty liver (Bobe et al., 2004; Ingvartsen, 2006). Moreover, we have recently shown that increased NEFA is associated with elevated plasma ceramides (Rico et al., 2015). Because plasma ceramide concentration was positively associated with liver lipid content in the current study (e.g. C24:0-ceramide, $r=0.4 ; P<0.05$ ), it is likely that ceramide synthesis and hepatic lipid accumulation are involved in a positive feedback cycle, by which increased fatty acid availability promotes ceramide synthesis in the liver, and the resulting ceramides can further promote uptake of circulating lipids. Future investigations should address this possibility by evaluating the relationships between liver lipid accumulation, ceramides and fatty acid transport.

Time-dependent changes were observed for several skeletal muscle ceramides and glycosylated ceramides, which increased during the transition from gestation to lactation (e.g. 
C16:0-, C22:0-ceramide, and 16:0-LacCer, $P<0.05)$. Overall, no significant differences in ceramide accumulation were observed between lean and overweight cows, however, the predominant $\mathrm{C} 24$ :0-ceramide tended to be lower in overweight cows, relative to lean $(P<0.1$; Figure 4-12). Because ceramides have been reported to accumulate in obese individuals (Haus et al., 2009), this result is unexpected. Although the reasons for this observation are unclear, we propose that, in contrast to obese humans, overweight cows during the transition from gestation to lactation suffer from decreased appetite and more severe negative energy balance, predisposing them to excessive lipolysis and increased muscle catabolism and weight loss. Under such metabolic state, it is conceivable that ectopic lipid accumulation, such as that of ceramide, is not maximized. Despite this paradoxical result, the observation that ceramides and glycosylated ceramides increased during peripartum demonstrates that increased ceramide accumulation occurs in this period, concomitant with near-maximal plasma NEFA content (Samii et al., JDS, under submission).

Considering that liver-derived ceramides can induce insulin resistance in monogastrics (Summers et al., 1998, Boon et al., 2013; Chavez et al., 2014), we hypothesized that circulating ceramides would be associated with direct measurements of insulin action. As expected, significant inverse associations between postpartum glucose disappearance during ITT and ceramides were detected, ranging 0.50 to $0.75(P<0.05$; Figure 4-14), with the strongest correlations observed for $\mathrm{C} 18$ :0-ceramide $(P<0.001)$. Importantly, the strong associations between ceramides and insulin sensitivity were observed during a period in which cows undergo negative energy balance, inflammation, and extensive lipomobilization. Whether similar observations can occur during established lactation and under positive energy balance is yet to be established. 
In addition to their effects on insulin sensitivity, ceramides mediate the apoptotic effects of fatty acids on pancreatic $\beta$ cells and can cause reduced expression of the insulin gene, resulting in reduced pancreatic insulin release (Shimabukuro et al., Sjoholm, 1998; Kleppe et al., 2003). Because insulin secretion usually decreases as parturition approaches (Rico et al., 2015), we were interested in evaluating the association between plasma insulin concentration and ceramide. An inverse relationship between glycosylated ceramides and insulin in plasma was detected for several glycosylated ceramides $(r=-0.26$ to $-0.41 ; P<0.001)$. Future investigations should determine whether ceramides are mechanistically involved in the peripartal decrease of insulin secretion from the pancreas.

Peripartal insulin resistance and lipolysis represent adaptive mechanisms that facilitate necessary shifts in nutrient metabolism to support the elevated glucose demands of lactation. Although ceramides have the ability to disrupt insulin signaling, their role in driving insulin resistance during the transition from gestation to lactation is still unknown. By examining ceramide accumulation and insulin sensitivity, we established that postpartum insulin resistance is associated with increased ceramide. Furthermore, using a model of increased adiposity, we demonstrated that overweight cows displayed elevated concentrations of circulating ceramides, however, their ability to induce insulin resistance remains unknown. Future research we will need to determine whether ceramide mediates insulin resistance in dairy cows, both physiologically and pathologically. If a causal relationship is established, factors contributing to excessive ceramide synthesis, such as inflammation and excessive adipose tissue mobilization, will require evaluation. Our data suggest that sphingomyelin hydrolysis can be a major contributor to ceramide synthesis. Therefore, special focus should be placed in the investigation of hepatic sphingomyelin metabolism and its modulation to regulate ceramide accrual during peripartum. 


\section{Conclusion}

Our results support the association between ceramides and insulin resistance in peripartal dairy cows. Moreover, the increased concentrations of ceramide and glycosylated ceramide in liver and plasma of overweight dairy cows demonstrate that elevated body fat accumulation contributes to the accumulation of these sphingolipids. Our targeted metabolomics approach revealed that sphingolipid metabolism changes dynamically during the transition from gestation to lactation. We conclude that temporal changes in sphingolipid metabolism results in active remodeling of the plasma sphingolipidome, and result in ceramide accumulation, independent of body fat content. We also conclude that ceramide tissue accumulation occurs progressively during the transition from gestation to lactation, and is associated with NEFA and sphingomyelin availability. Our data show that ceramide accumulation during peripartum is associated with the homeorhetic reduction of insulin action postpartum, supporting a role for ceramides in facilitating the physiological adaptations during this period. To confirm this possibility, further studies should investigate whether ceramide mediates the development of insulin resistance in dairy cattle. Furthermore, the role of inflammation in driving sphingomyelin hydrolysis will need to be investigated, as modulation of excessive ceramide accumulation could potential improve cow health during peripartum. 
Table 4-1. Ingredients and nutrient composition of diets fed to lean and overweight cows during the transition from late pregnancy to early lactation.

\section{Diets}

\begin{tabular}{lcc}
\cline { 2 - 3 } Ingredients, \% of DM & Prepartum & Lactation \\
\hline Corn silage & 30.7 & 42.4 \\
Mixed grass haylage & 28.4 & 7.0 \\
Mixed grass hay & 13.2 & 2.1 \\
Dry ground corn & - & 14.4 \\
Prepartum mix ${ }^{1}$ & 13.9 & - \\
Lactation mix A ${ }^{2}$ & - & 14.0 \\
Aminoplus & 9.0 & 4.7 \\
Cottonseed with lint & - & 4.5 \\
Sugar cane syrup & - & 3.6 \\
Lactation mix B & - & 4.5 \\
Close-up supplement & 4.7 \\
Rumensin & 4.8 & 2.7 \\
Nutrient Composition & - & 0.1 \\
DM, \% & & \\
NDF, \% of DM & 55.5 & 51.6 \\
$\quad$ Forage NDF, \% of DM & 43.7 & 37.6 \\
Forage NDF, \% of NDF & 90.5 & 25.1 \\
ADF, \% of DM & 32 & 66.7 \\
CP, \% of DM & 12.2 & 25.2 \\
Starch, \% of DM & 14.1 & 16.5 \\
Ether extract, \% of DM & 3.2 & 21.2 \\
Ash, \% of DM & 7.5 & 4.3 \\
\hline Mix contned & $47 \%$ commecia & \\
\hline
\end{tabular}

${ }^{1}$ Mix contained 27\% commercial dry cow mix with Animate (Phibro Animal Health Corp., Teaneck, NJ), $17.3 \%$ ground corn, $17.3 \%$ crimped oats, $13 \%$ corn distillers, $12.9 \%$ soybean meal, $4.3 \%$ calcium carbonate, $4.3 \%$ calcium sulfate, 2.6\% Omnigen AF (Phibro Animal Health Corp.), 1.1 Monocalcium phosphate, and < $1 \%$ of each of the following: Sel-plex 600 (Alltech Biotechnology, Nicholasville, KY) and vitamin E. ${ }^{2}$ Mix contained $33.6 \%$ citrus pulp, $19 \%$ soybean meal. $15.6 \%$ canola meal, $14 \%$ soybean hulls, $4.5 \%$ calcium carbonate, $4.5 \%$ sodium bicarbonate, $2.8 \%$ urea, $2.7 \%$ fat, $2.4 \%$ sodium chloride, and $<1 \%$ from each of the following: Monocalcium phosphate, biotin, and Rumensin 90 (Elanco Animal Health, Greenfield, IN). ${ }^{3}$ Mix contained 43.6\% Fermenten (Church and Dwight Co., Princeton, NJ), $21.8 \%$ calcium carbonate, $10.8 \%$ soybean hulls, $7.8 \%$ Mintrex blend (Novus International Inc., St. Charles, MO), 5.2\% blood meal, 3.5\% magnesium oxide, 2.6\% Celmanax (Vi-COR, Mason City, IA), $2.6 \%$ Omnigen (Phibro Animal Health Corp.), and $<1 \%$ of each of the following: vitamin E, selenium selenite, and selenium yeast $600 .{ }^{4} \mathrm{Mix}$ contained $74.5 \%$ ground oats, $15.3 \%$ commercial amino acid, $8 \%$ Reashure (Balchem Encapsulates, Slate Hill, NY), and $<1 \%$ of each of the following: vitamin E and Niashure (Balchem Corporation, New Hampton, NY). ${ }^{5}$ Rumensin for dairy included at 4,890 mg/kg. 


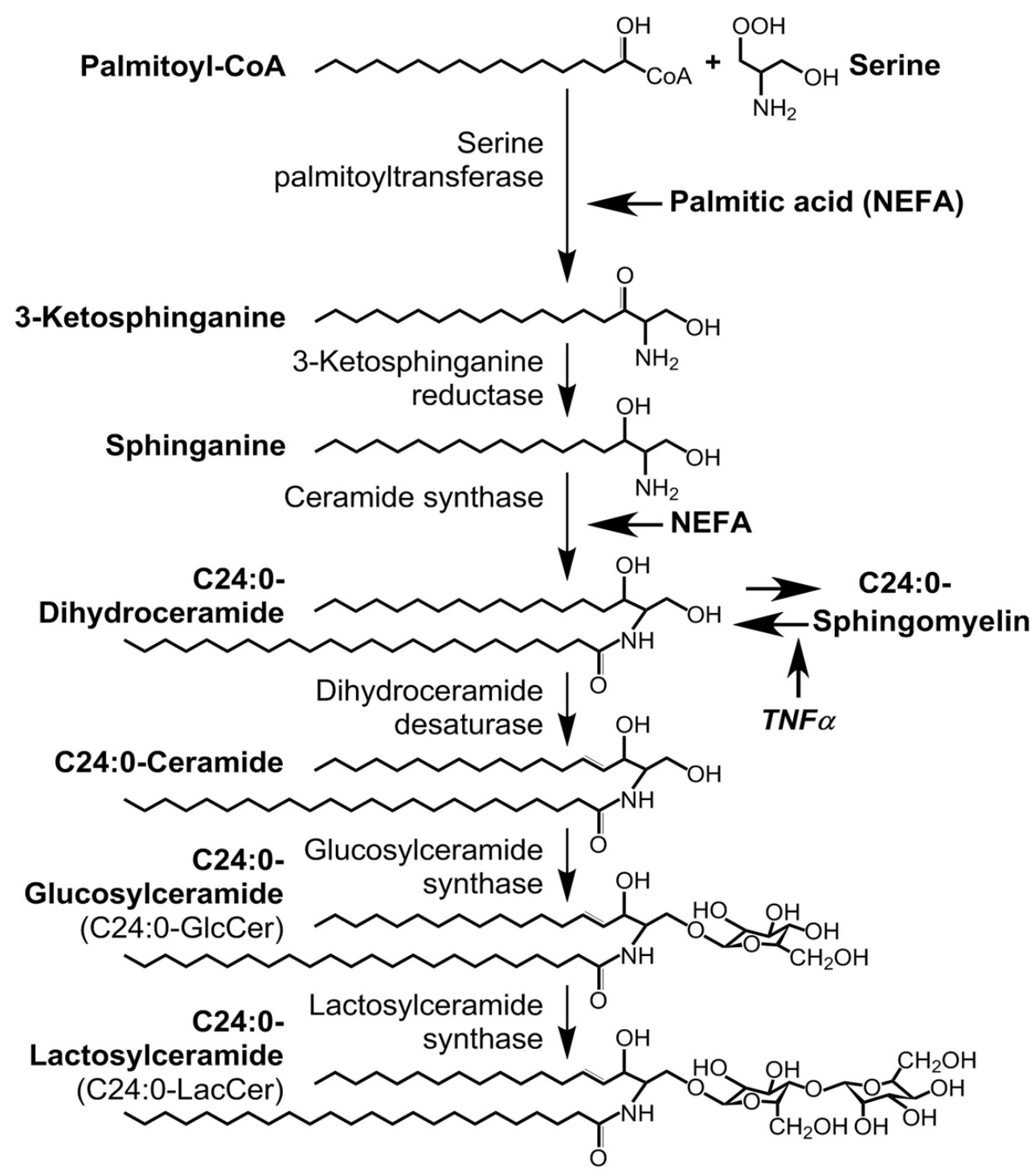

Figure 4-1. Major pathways of ceramide synthesis.

De novo ceramide is initiated by the condensation of palmitoyl-CoA and serine, a pathway upregulated by saturated fatty acids (SFA). Sphingomyelin hydrolysis results in ceramide synthesis, a process induced by the inflammatory cytokine TNF $\alpha$. Sphingolipids have various acyl chain lengths and can differ in carbon length and degree of saturation. For simplicity, C24:0-linked sphingolipids and glycosphingolipids are illustrated. Monohexosylceramides (GlcCer) consist of a single sugar residue, either glucose or galactose (galactosylceramide is not shown). 


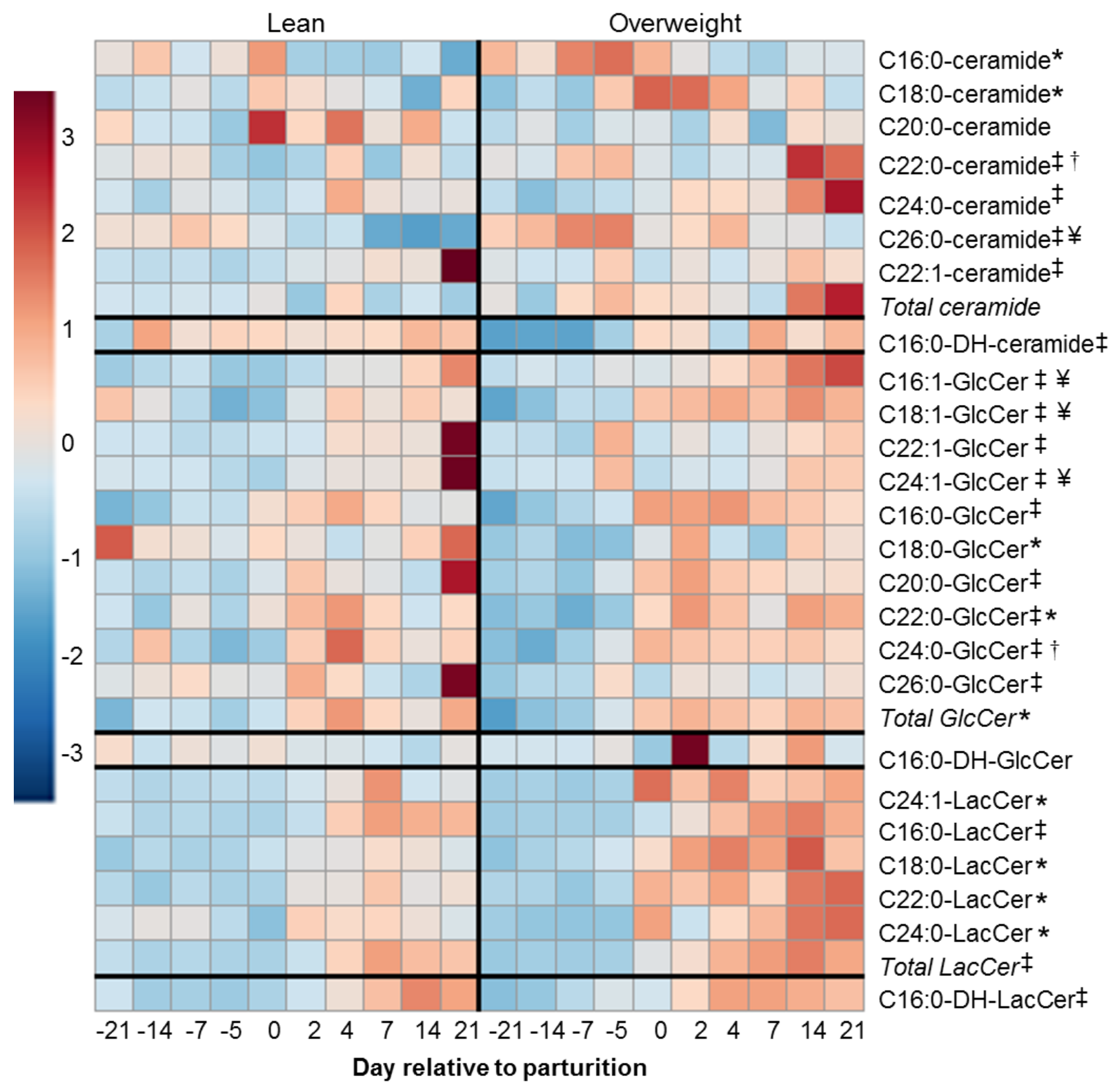

Figure 4-2. Plasma ceramides, monohexosylceramides (GlcCer), and lactosylceramides (LacCer) are elevated in overweight dairy cows transitioning from gestation to lactation.

For visualization purposes, the heat map represents relative concentrations as high (red) or low (blue). The heat map was generated using MetaboAnalyst 3.0 (Xia et al., 2015). Data are represented as least squares means and their standard errors. $\ddagger$, main effect of day relative to parturition, $P<0.05 ; ¥, P<0.1$. *, Treatment $\times$ Day interaction, $P<0.05 ; \uparrow, P<0.10$. DH: dihydro. 


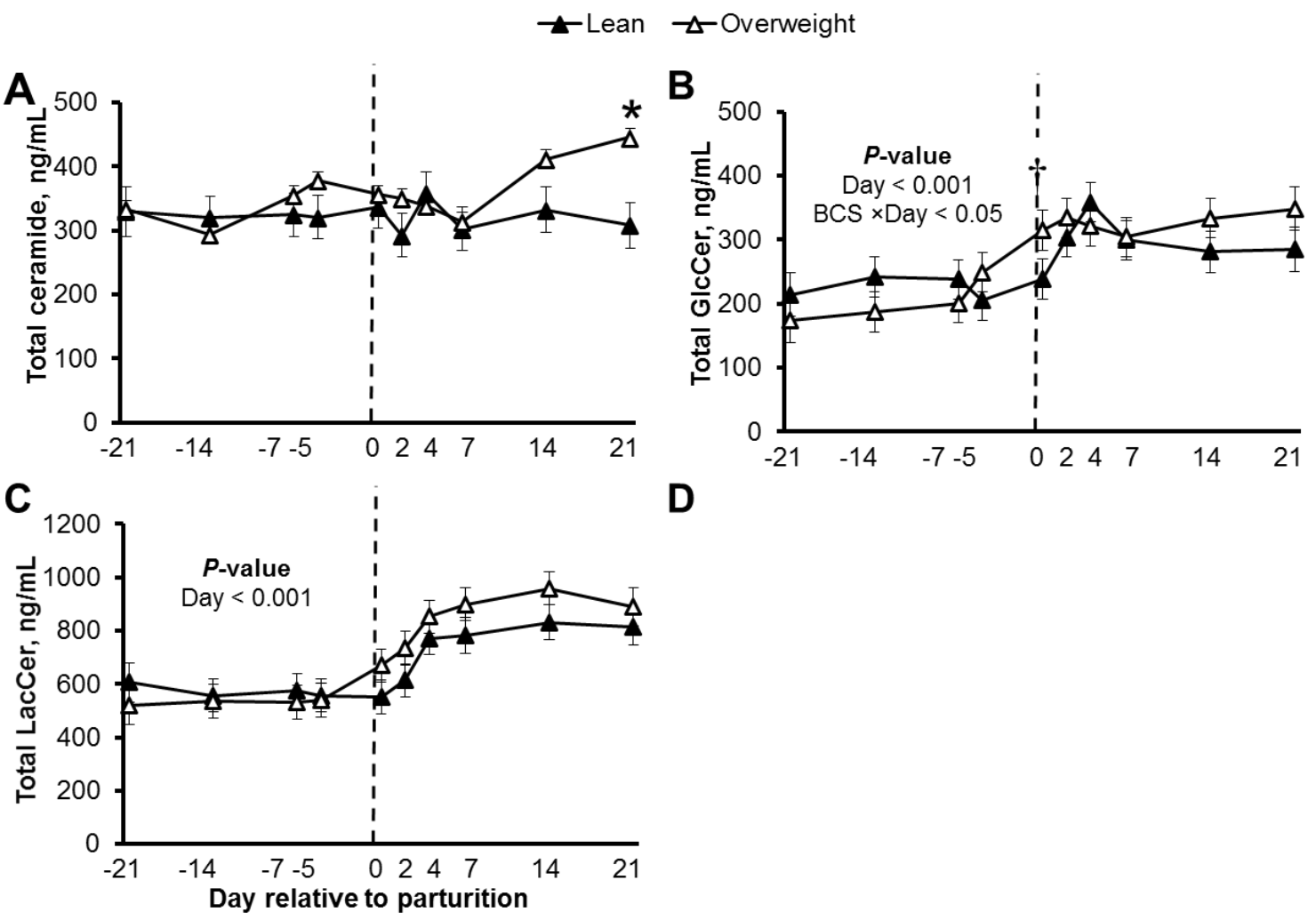

Figure 4-3. Plasma ceramides change dynamically during the transition from gestation to lactation.

Plasma concentration of plasma total (A) ceramide, (B) GlcCer, and (C) LacCer in lean and overweight transition dairy cows. Data are represented as least squares means and their standard errors. * $P<0.05 ;+, P<0.10$. Glc: Monohexosylceramide, LacCer: lactosylceramide, 
$\neg$-Lean $\triangle-$ Overweight
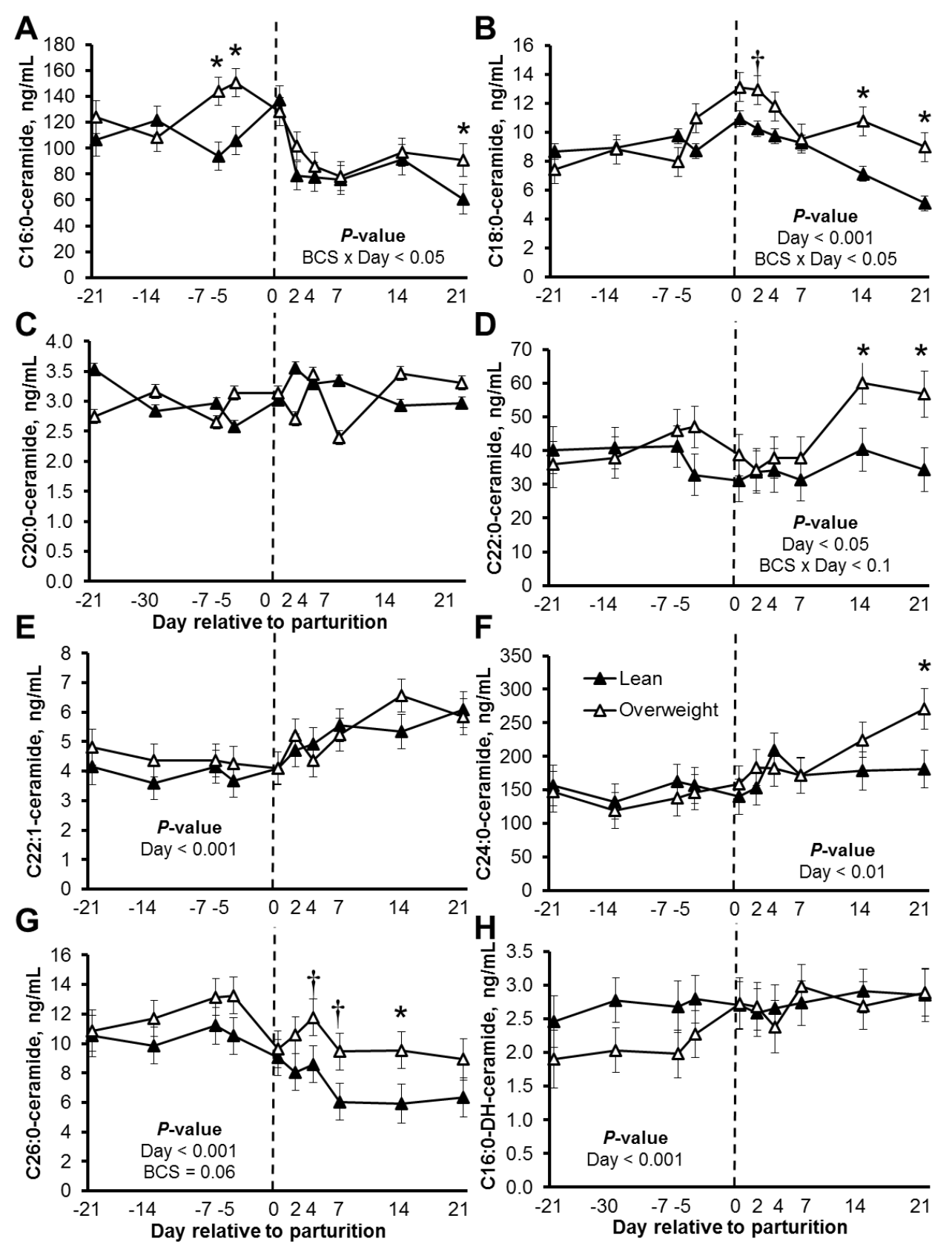

Figure 4-4. Plasma ceramides are elevated in overweight cows during the transition from gestation to lactation.

Plasma concentration of (A) C16:0-, (B) C18:0-, (C) C20:0-, (D) C22:0-, (E) C22:1-, (F) C24:0-, (G) C26:0-ceramide, and (H) C16:0-DH-ceramide in lean and overweight transition dairy cows. Data are represented as least squares means and their standard errors. ${ }^{*}, P<0.05 ;+, P<0.10$. DH: dihydro. 

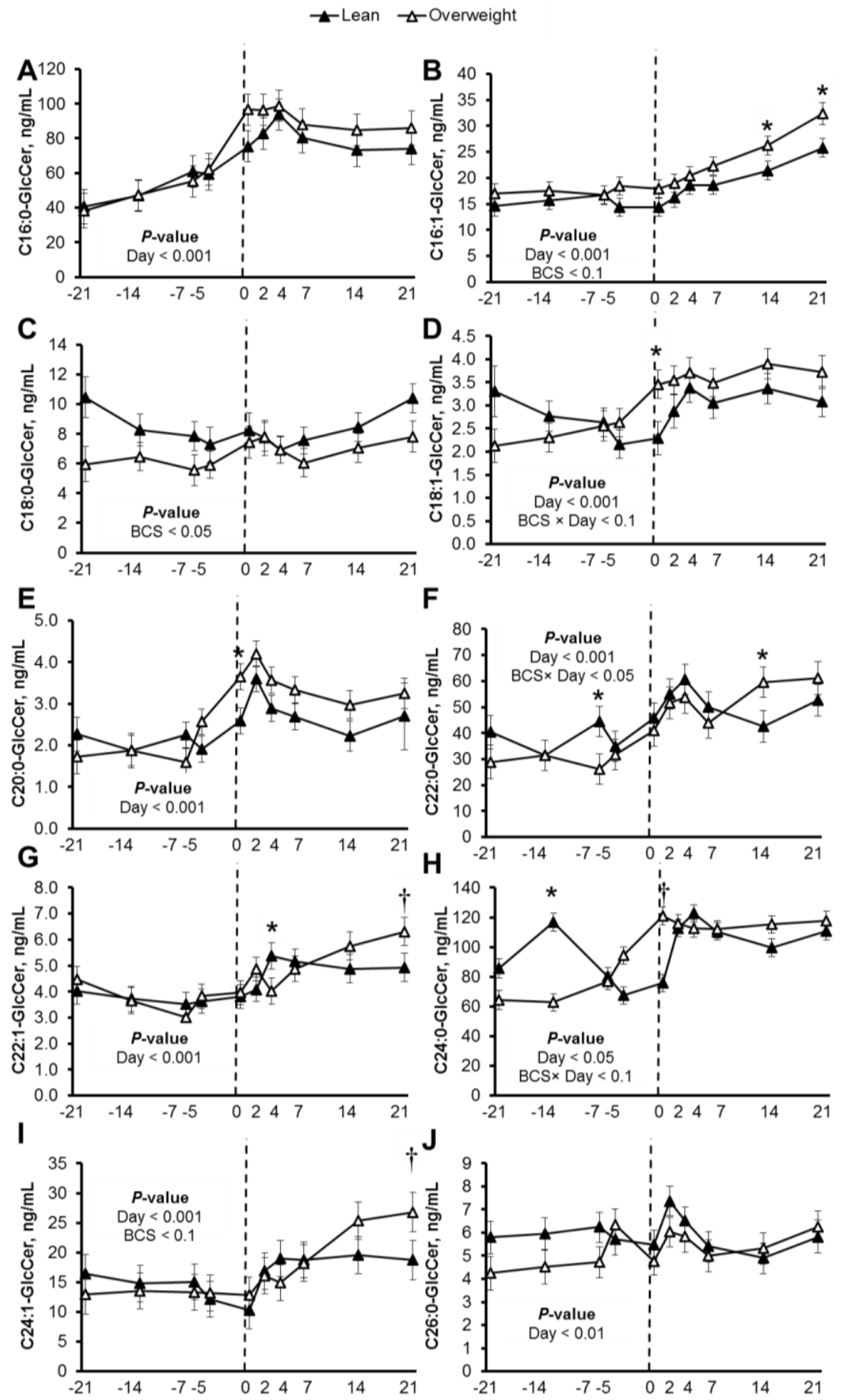

Figure 4-5. Plasma concentrations (ng/mL) of monohexosylceramides (GlcCer) in lean and overweight cows transitioning from gestation to lactation.

Plasma (A) C16:0-, (B) C16:1-, (C) C18:0-, (D) C18:1-, (E) C20:0-, (F) C22:0-, (G) C22:1, (H) C24:0-, (I) C24:1-, and (J) C26:0-GlcCer, in lean and overweight transition dairy cows. Data are represented as least squares means and their standard errors. ${ }^{*}, P<0.05 ; \uparrow, P<0.10$. 
$\neg$ Lean $\rightarrow-$ Overweight
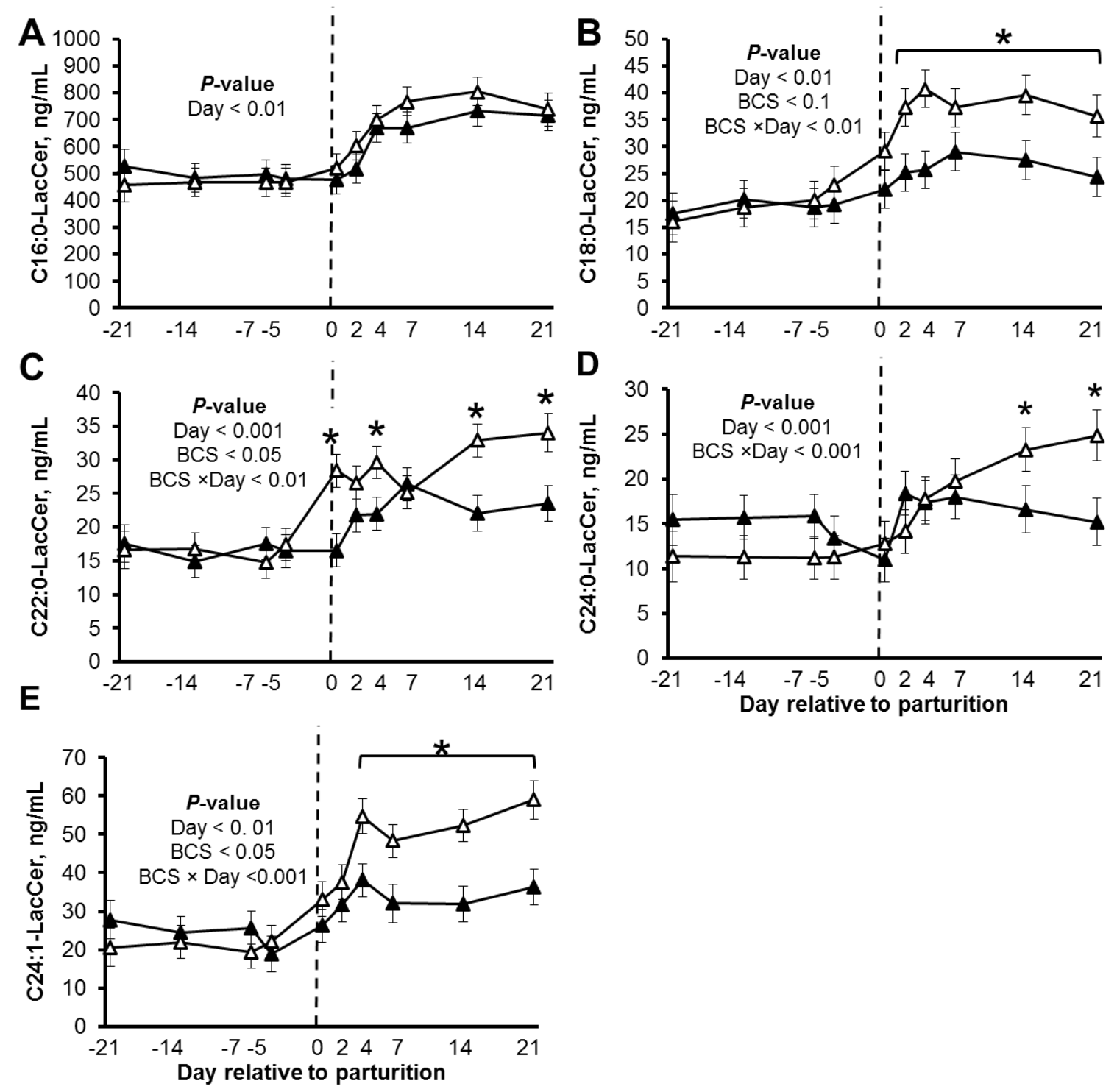

Figure 4-6. Plasma concentrations (ng/mL) of lactosylceramides (LacCer) in lean and overweight cows transitioning from gestation to lactation.

Plasma concentrations of (A) C16:0-, (B) C18:0-, (C) C22:0-, (D) C24:0-, (E) C20:0-, (F) C22:0, (G) C22:1, (H) C24:0-, (I) C24:1-, and (J) C26:0-LacCer in lean and overweight transition dairy cows. Data are represented as least squares means and their standard errors. ${ }^{*}, P<0.05$. 


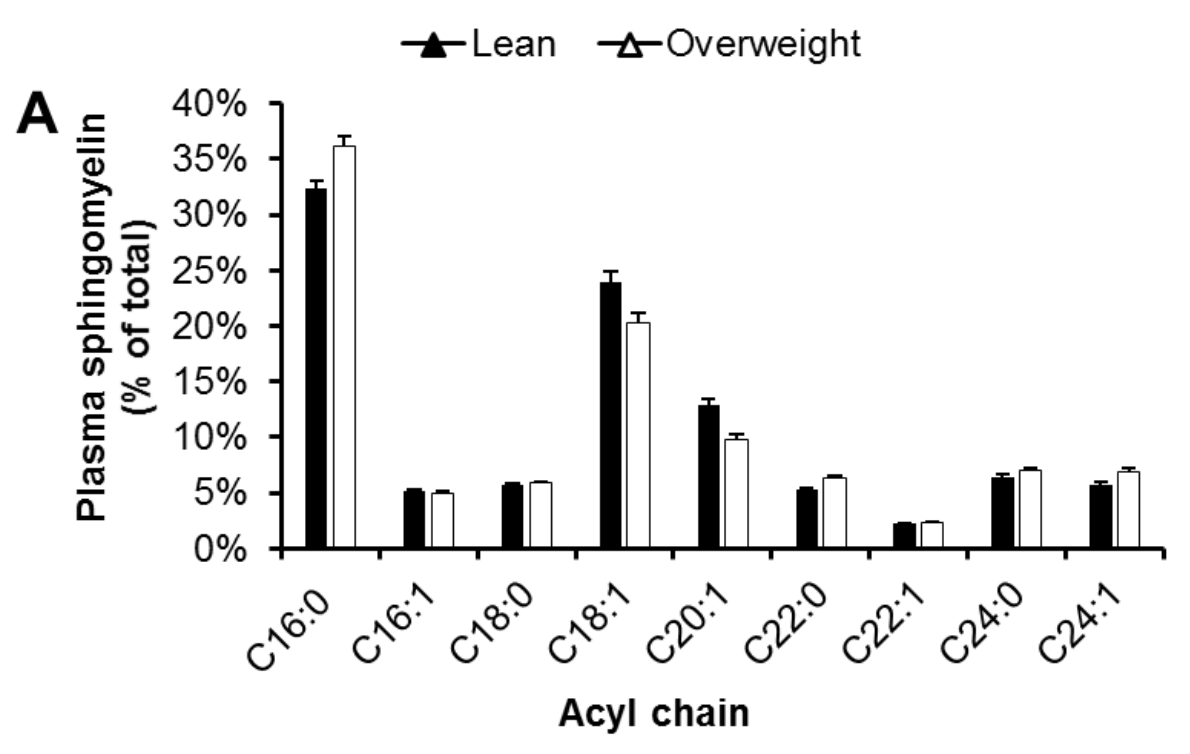

B

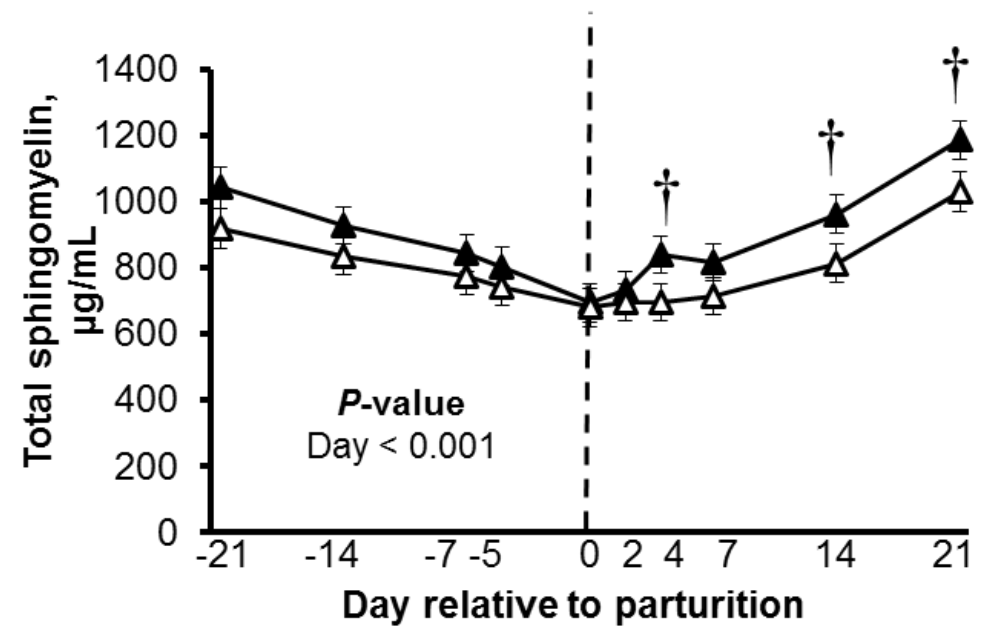

Figure 4-7. Plasma sphingomyelin profile during the transition from gestation to lactation.

(A) Plasma profile of sphingomyelin and (B) temporal changes of plasma sphingomyelin in lean and overweight cows. Data are represented as least squares means and their standard errors. ${ }^{*}, P<$ $0.05 ; \dagger, P<0.10$. 


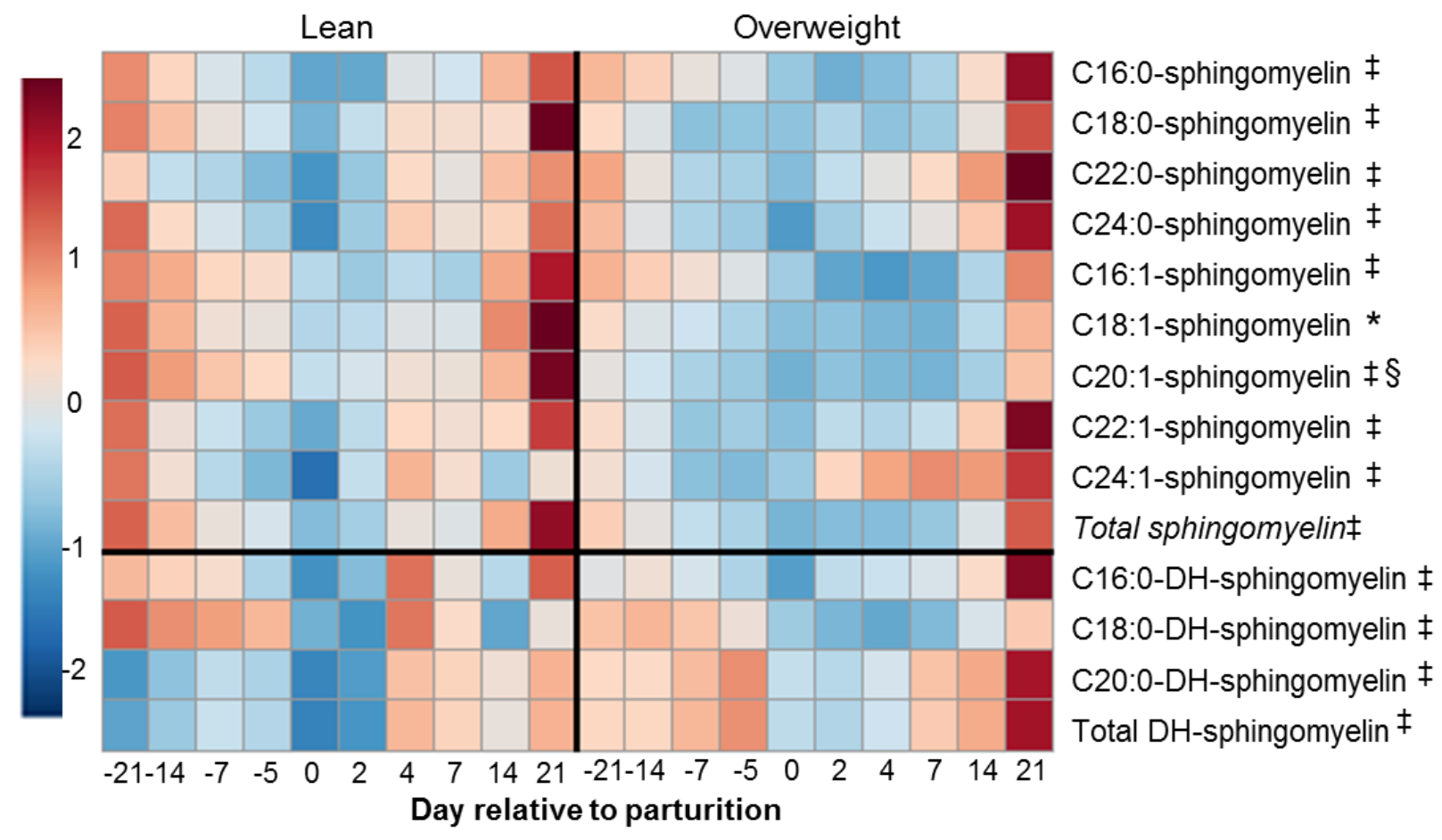

Figure 4-8. Plasma sphingomyelins change dynamically in dairy cows transitioning from gestation to lactation.

For visualization purposes, the heat map represents relative concentrations as high (red) or low (blue). The heat map was generated using MetaboAnalyst 3.0 (Xia et al., 2015). Data are represented as least squares means and their standard errors. $¥$, main effect of day relative to parturition, $P<0.05$. $\S$, main effect of adiposity (BCS), $P<0.05$; *, Treatment $\times$ Day interaction, $P<0.05 ; \dagger, P<0.10$. DH: dihydro. 


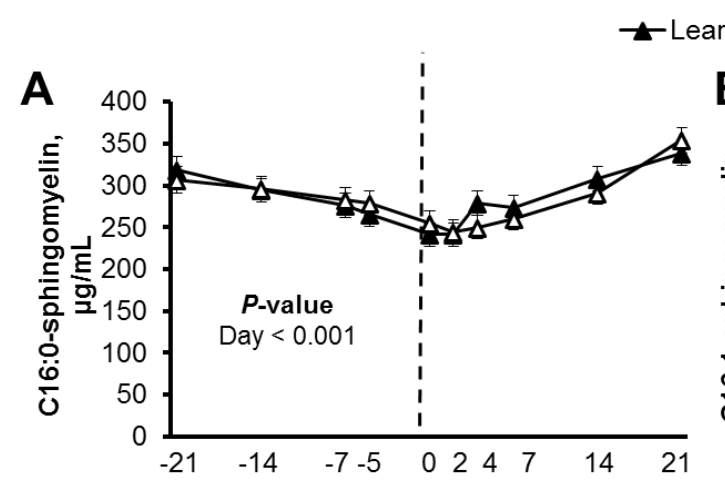

$-\Delta$ Overweight
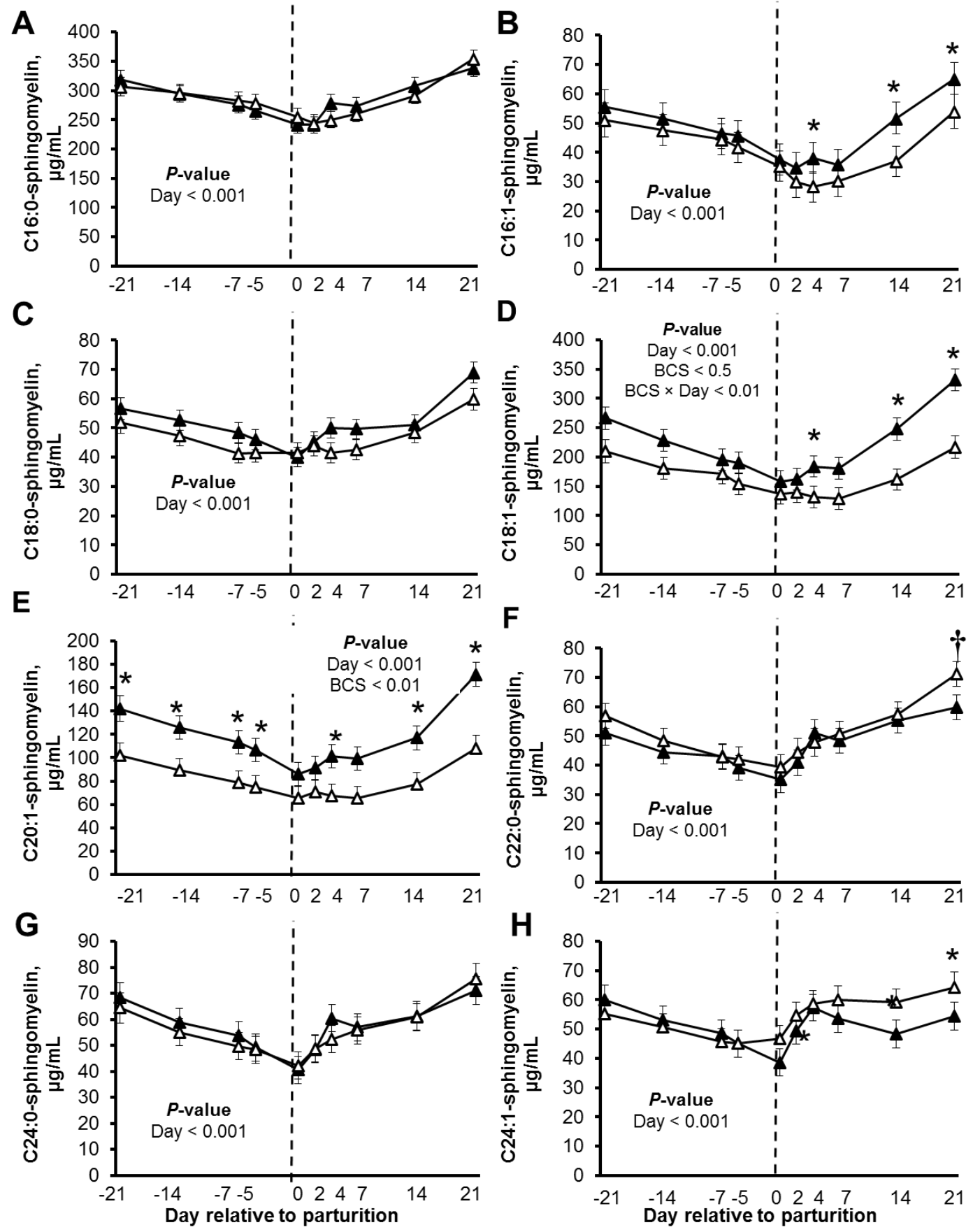

Figure 4-9. Plasma sphingomyelins decrease toward parturition and increase postpartum.

Plasma concentrations $(\mu \mathrm{g} / \mathrm{mL})$ of (A) C16:0-, (B) C16:1-, (C) C18:0-, (D) C18:1-, (E) C20:0-, (F) C22:0-, (G) C24:0-, and (H) C24:1-sphingomyelin in lean and overweight transition dairy cows. Data are represented as least squares means and their standard errors. ${ }^{*}, P<0.05 ; \dagger, P<$ 0.10 . 

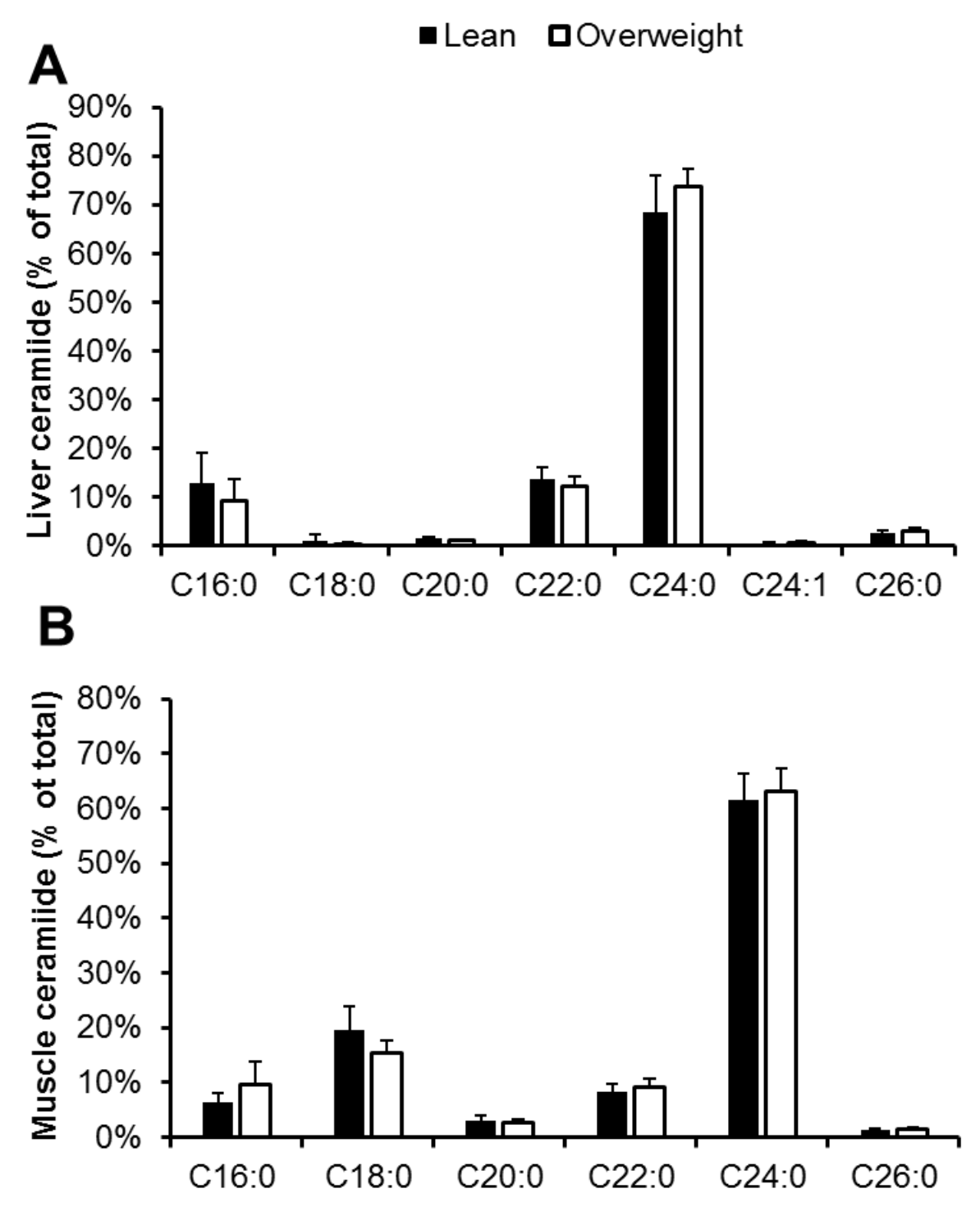

Figure 4-10. Ceramide profile of liver and skeletal muscle at $\mathrm{d} 4$ postpartum.

Ceramide profile in (A) liver and $(\mathbf{B})$ skeletal muscle. Data are represented as means and their standard deviations. 


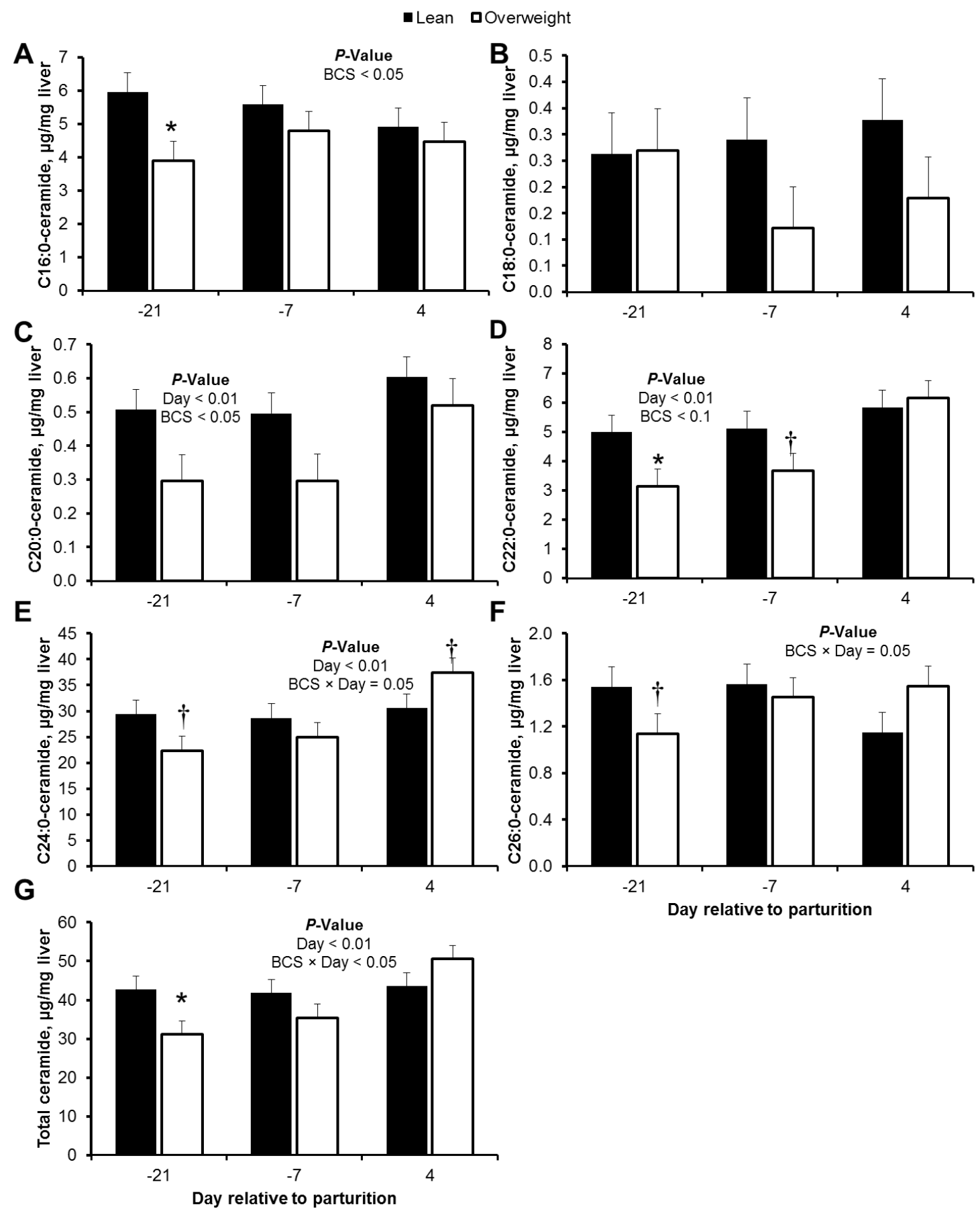

Figure 4-11. C24:0-ceramide and total ceramide hepatic accumulation increase in overweight cows during the transition from gestation to lactation.

Hepatic concentrations ( $\mu \mathrm{g} / \mathrm{mg}$ liver) of (A) C16:0-, (B) C18:0-, (C) C20:0-, (D) C22:0-, (E) C24:0-, (F) C26:0-, and (G) total ceramide in lean and overweight transition dairy cows. Data are represented as least squares means and their standard errors. ${ }^{*}, P<0.05 ; \uparrow, P<0.10$. 


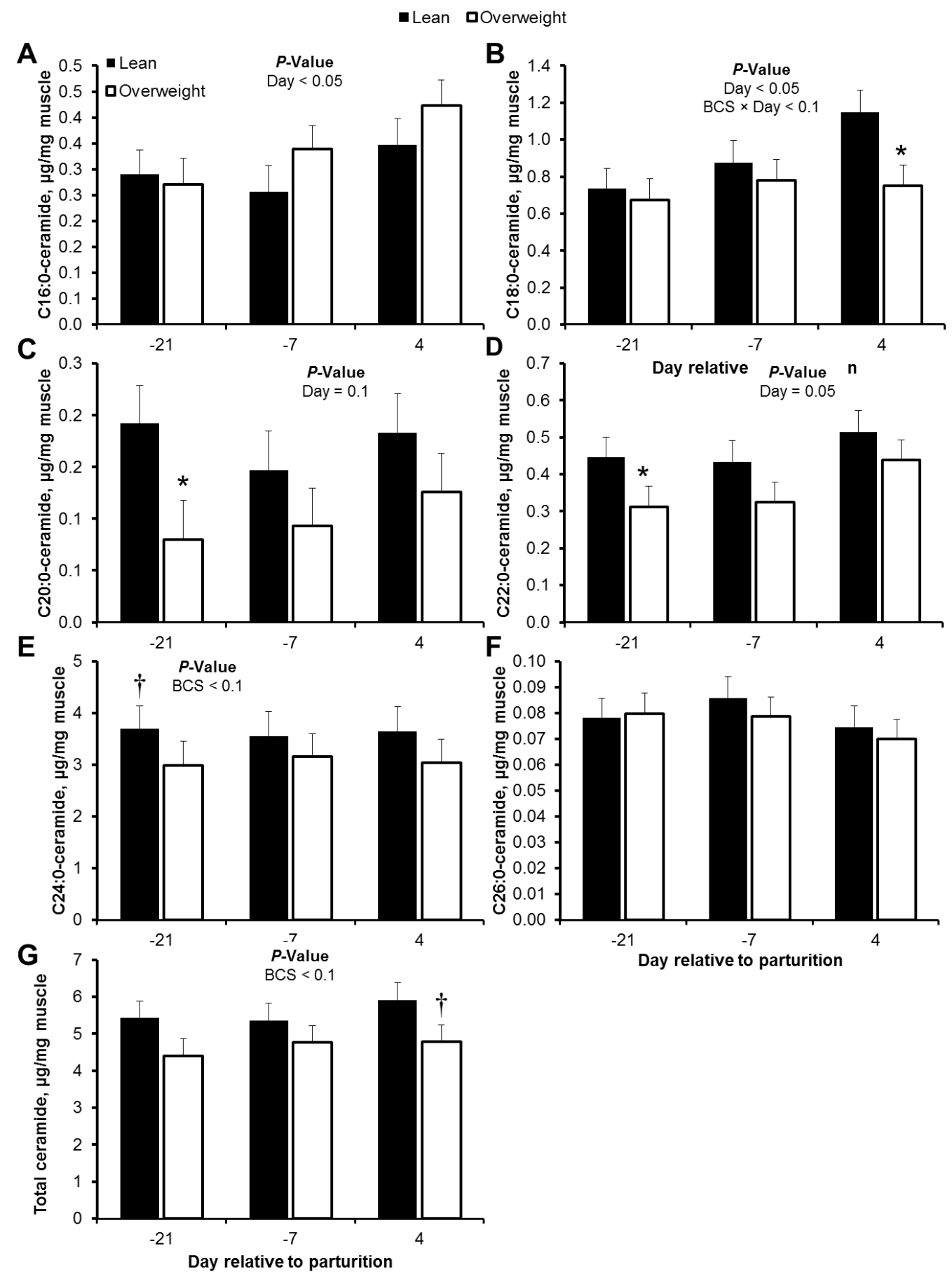

Figure 4-12. C24:0-ceramide and total ceramide content of skeletal muscle during the transition from gestation to lactation.

Skeletal muscle concentrations ( $\mu \mathrm{g} / \mathrm{mg}$ tissue) of (A) C16:0-, (B) C18:0-, (C) C20:0-, (D) C22:0, (E) C24:0-, (F) C26:0-, and (G) total ceramide in lean and overweight transition dairy cows. Data are represented as least squares means and their standard errors. ${ }^{*}, P<0.05 ; \uparrow, P<0.10$. 

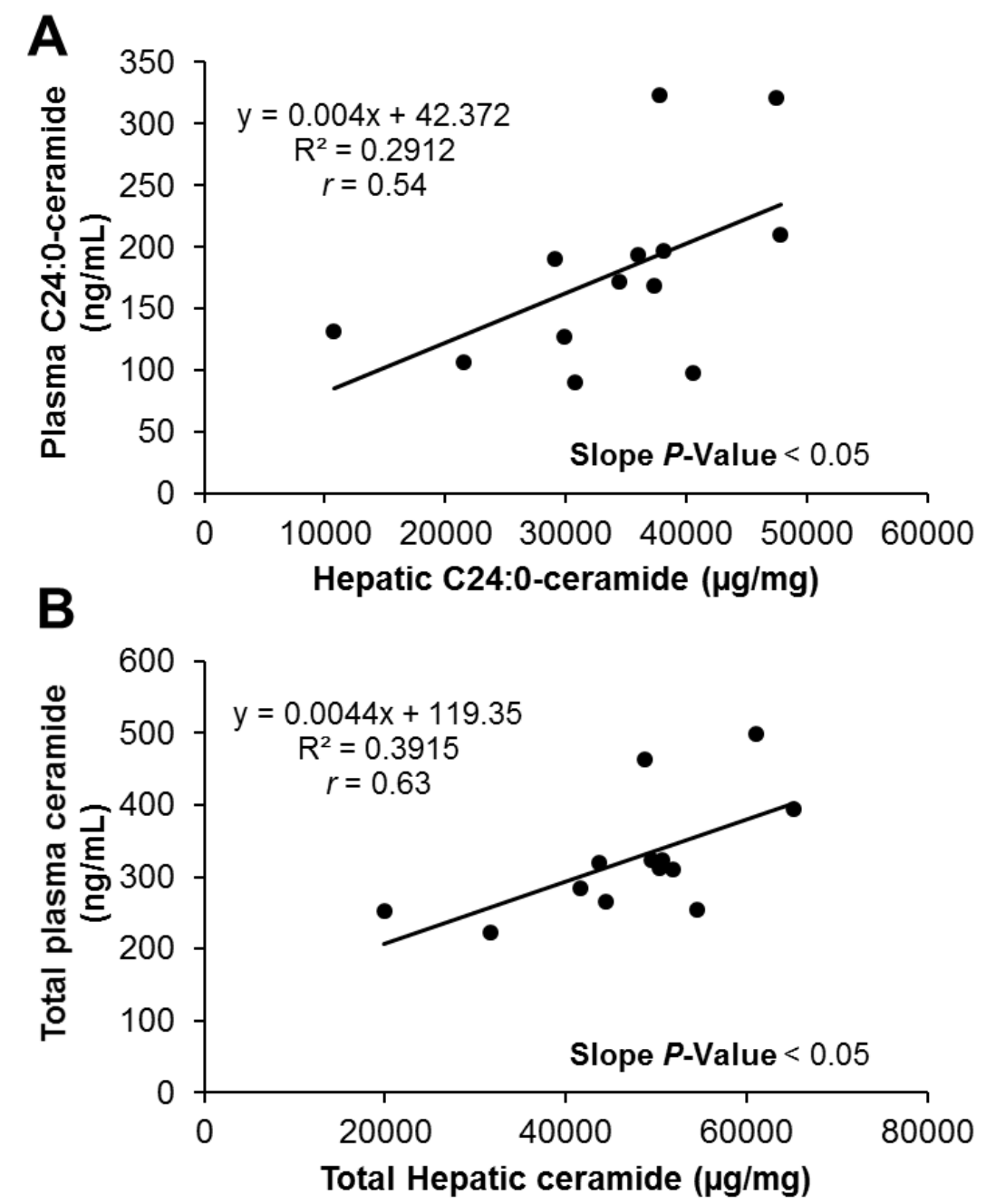

Figure 4-13. Plasma ceramide content is associated with hepatic ceramide accumulation postpartum.

Simple linear regression analysis between plasma and liver concentrations of (A) C24:0-ceramide and (B) total ceramide in lean and overweight dairy cows at d 4 postpartum. 


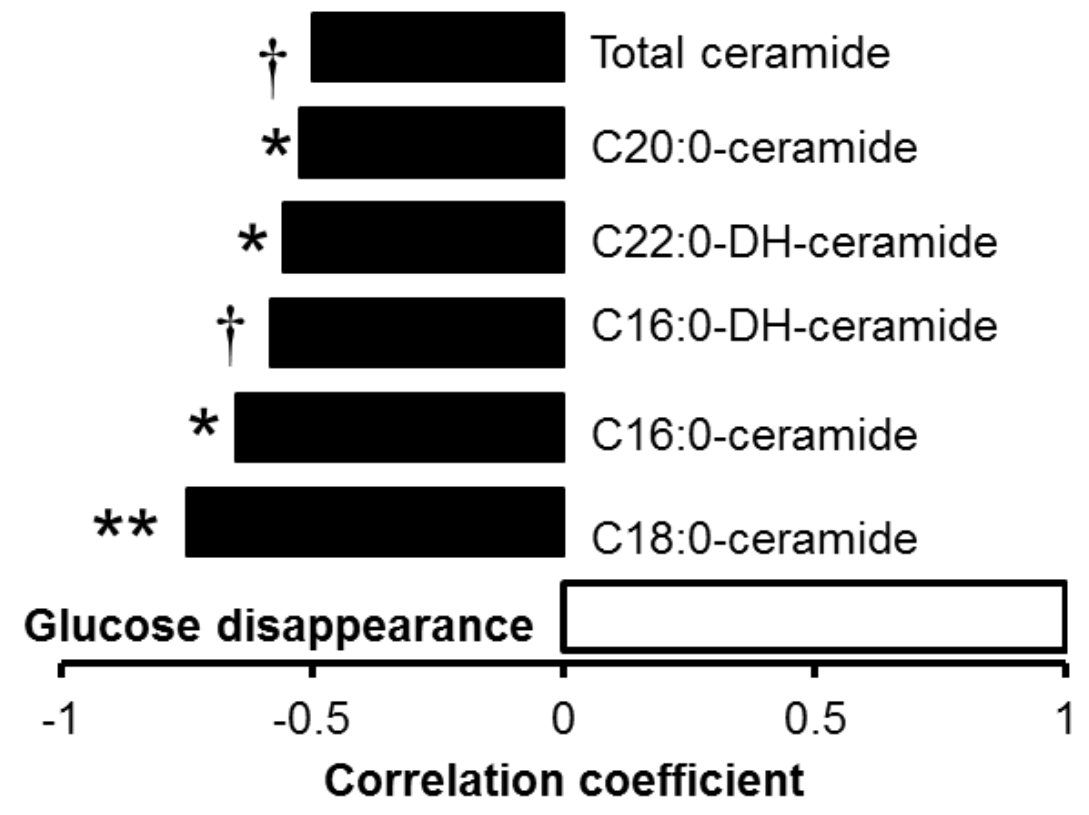

Figure 4-14. Circulating ceramides are inversely associated glucose disappearance during an insulin challenge at $\mathrm{d} 4$ postpartum.

Pearson's ranked correlations of circulating ceramides and glucose disappearance during ITT. $* *, P<0.01 ; *, P<0.05 ; \dagger, P<0.10$. ITT: Insulin tolerance test; DH: Dihydro. 


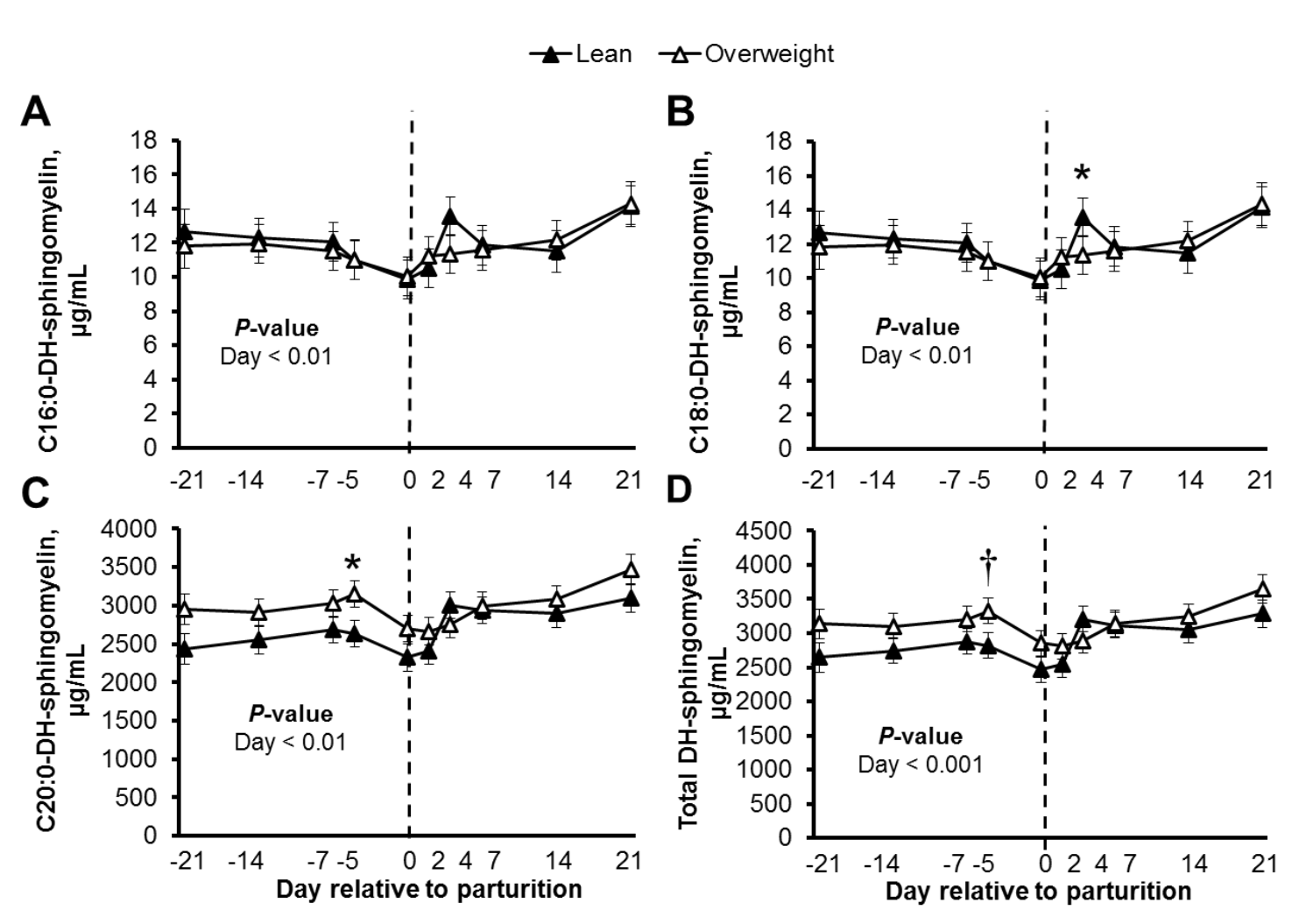

Figure 4-15. Supplemental Figure 1. Plasma dihydro-sphingomyelins (DH) decrease toward parturition and increase postpartum.

Plasma concentrations $(\mu \mathrm{g} / \mathrm{mL})$ of (A) C16:0-, (B) C18:0-, (C) C20:0-, and (D) total sphingomyelin in lean and overweight transition dairy cows. Data are represented as least squares means and their standard errors. ${ }^{*}, P<0.05 ; \uparrow, P<0.10$. 


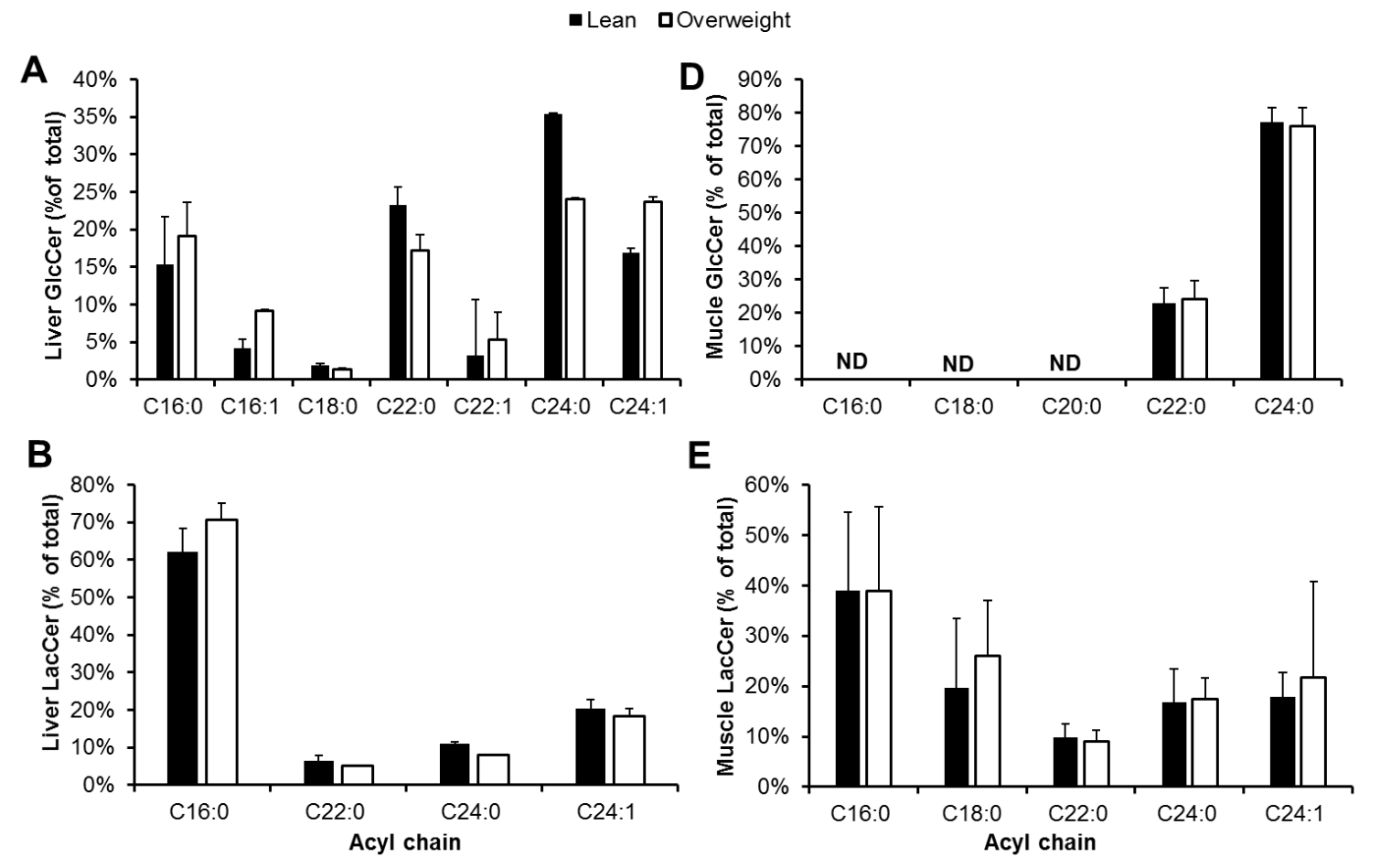

Figure 4-16. Supplemental Figure 2. Monohexosylceramide (GlcCer) and lactosylceramide (LacCer) profile of liver and skeletal muscle at $\mathrm{d} 4$ postartum.

Hepatic profile of (A) GlcCer and (B) LacCer, and skeletal muscle profile of (C) GlcCer and (D) LacCer. Data are represented as means and their standard deviations. 


\title{
Chapter 5
}

\section{PALMITIC ACID FEEDING INCREASES CERAMIDE AVAILABILITY IN ASSOCIATION WITH INCREASED MILK YIELD, NEFA AVAILABILITY, AND ADIPOSE TISSUE RESPONSIVENESS TO A GLUCOSE CHALLENGE.}

\begin{abstract}
Reduced insulin action is a key adaptation that facilitates glucose partitioning to the mammary gland for milk synthesis during early lactation, an adaptation occurs in parallel with enhanced adipose tissue lipolysis. The progressive recovery of insulin sensitivity as cows advance toward late lactation is accompanied by reductions in circulating non-esterified fatty acids (NEFA) and milk yield. Because palmitic acid can promote insulin resistance in monogastrics through sphingolipid ceramides-dependent mechanisms, C16:0 feeding may enhance milk production by restoring homeorhetic responses. We hypothesized that $\mathrm{C} 16: 0$ feeding would enhance ceramide availability in mid-lactation dairy cattle, and ceramide would be positively associated with milk yield. Twenty multiparous mid-lactation Holstein cows were enrolled in a study consisting of a 5 d covariate, $49 \mathrm{~d}$ treatment, and $14 \mathrm{~d}$ post-treatment period. All cows received a common sorghum silage-based diet and were randomly assigned to a diet containing no supplemental fat (control; $\mathrm{n}$ $=10 ; 138 \pm 45$ DIM) or C16:0 at 4\% of ration DM (PALM; 98\% C16:0; $\mathrm{n}=10 ; 136 \pm 44 \mathrm{DIM})$. Blood and milk were collected at routine intervals. Liver and skeletal muscle tissue were biopsied at d 47 of treatment. Intravenous glucose tolerance tests (300 mg/kg of body weight (BW); GTT) were performed at $\mathrm{d}-1,21$, and 49 relative to start of treatment. The plasma and tissue concentrations of ceramide and glycosylated ceramide were determined using liquid chromatography-based mass spectrometry. Data were analyzed as repeated measures using a mixed model with fixed effects of treatment and time, and milk yield served as a covariate. Cows
\end{abstract}


fed PALM experienced an increase in milk yield, energy-corrected milk (ECM), and milk fat yield. The most abundant plasma and tissue sphingolipids detected were C24:0-ceramide, C24:0monohexosylceramide (GlcCer), and C16:0-lactosylceramide (LacCer). Plasma concentrations of total ceramide and GlcCer decreased as lactation advanced, and ceramide and GlcCer were elevated in cows fed PALM. Feeding PALM increased hepatic ceramide levels, a response not observed in skeletal muscle tissue. Plasma ceramides (e.g., C24:0-ceramide) were positively correlated with plasma NEFA and milk yield, and inversely correlated with NEFA disappearance following a glucose challenge. Our data demonstrate a remodeled plasma and hepatic sphingolipidome in dairy cows fed PALM.

\section{Introduction}

The development of insulin resistance in adipose and skeletal muscle tissues enables the dairy cow to partition glucose toward the mammary gland during early lactation (Bell, 1995; Bell and Bauman, 1997). As a consequence, a decrease in insulin sensitivity can enhance the mobilization of non-esterified fatty acids (NEFA) from adipose tissue (Contreras et al., 2010; Zachut et al., 2013). NEFA can undergo mitochondrial $\beta$-oxidation in peripheral tissues, and reesterification in the mammary gland to contribute to milk fat synthesis. These coordinated metabolic adaptations support energy demand as milk production rapidly increases following parturition. Beyond peak milk yield, insulin sensitivity improves and the lipolytic release of NEFA decreases as the cow progresses toward late lactation and milk production steadily declines (McNamara and Hillers, 1986; Bell and Bauman, 1997).

A causal relationship between enhanced FA availability and the development of insulin resistance in dairy cattle has been repeatedly observed (Pires et al., 2007a, b; Rico et al., 2015). 
For instance, the intravenous infusion of tallow emulsion or suppressing adipose tissue lipolysis during feed restriction can improve insulin responsiveness (Pires et al., 2007a; b). In type 2 diabetic monogastric animal models, sustained hyperlipidemia shifts FA processing toward the hepatic synthesis and lipoprotein secretion of sphingolipid ceramide (Watt et al., 2012; Boon et al., 2013). Interestingly, de novo ceramide synthesis is controlled by the availability of saturated palmitoylCoA and the activation of serine palmitoyltransferase-1 (SPT1; Figure 5-1; Watt et al., 2012). The accrual of ceramide can antagonize insulin-stimulated glucose uptake by inhibiting the phosphorylation of protein kinase B (Akt; Summers et al., 1998; Chavez et al., 2003). With the advent of mass spectrometry-based metabolomics, sphingolipid ceramide and monohexosylceramide (glucosylceramide or galactosylceramide; GlcCer) have emerged as predicative biomarkers for the development of insulin resistance (Chavez et al 2014). Similar to the progression of the diabetic state in humans (Kautzky-Willer et al., 1997), we recently discovered that the decline in insulin sensitivity with the onset of lactation occurs concomitantly with the accumulation of ceramide in plasma (Rico et al., 2015). For example, we and others have observed a positive relationship between the availability of FA and the sphingolipid subspecie C24:0-ceramide in plasma, and the severity of insulin resistance (Haus et al., 2009; Rico et al., 2015); however, the relationship between FA and ceramide supply, and milk production in context of homeorhetic nutrient partitioning have not be evaluated in the mid-lactation dairy cow.

Feeding dairy cows diets containing supplemental SFA is a common nutritional approach to increase energy intake and milk yield during mid-lactation (Lock, et al., 2013; Rico et al., 2014a, b). Highly enriched SFA supplements are utilized by dairy producers because of their minimal effects on rumen microbial activity (Palmquist and Jenkins, 1980; Jenkins, 1993; Maia et al., 2010) and because of their beneficial milk production response as compared with unsaturated FA (UFA) 
supplementation (Christensen et al., 1994; Relling and Reynolds, 2007), particularly in highproducing dairy cows (Rico et al., 2014b). Although SFA feeding increases dietary energy, favorable lactation outcomes may not completely depend on the energy content of the SFA. For instance, feeding palmitic acid (C16:0) increases energy-corrected milk and milk fat yield when compared with C18:0 supplementation (Rico et al., 2014a). It is accepted that long-chain SFA are antagonists of insulin action in monogastric animals (Boden, 1997; Funaki, 2009), a response that is mediated by ceramide-dependent Akt inactivation (Summers et al., 1998; Chavez et al., 2003). Because SPT1 upregulation is dependent upon palmitoyl-CoA availability (Figure 5-1), feeding mid-lactation dairy cows C16:0 may upregulate de novo ceramide synthesis. Humans consuming a diet high in C16:0, relative to oleic acid, display increased circulating levels of ceramide (Kein et al., 2013), resembling upregulated ceramide synthesis observed in subjects diagnosed with type 2 diabetes (Haus et al., 2009). Although we and others have not observed changes in systemic glucose tolerance in dairy cows fed C16:0 when compared with unsupplemented control cows (Piantoni et al., 2013; Mathews et al., JDS Submitted), we have observed a decrease in glucosestimulated NEFA disappearance in mid-lactation cows fed C16:0 (Mathews et al., JDS Submitted), suggesting the possibility of localized adipose tissue insulin resistance with C16:0 feeding. The unexplored metabolic fates for absorbed C16:0, and the associative and functional role of ceramide to modify insulin sensitivity in response to $\mathrm{C} 16: 0$ supplementation merits attention.

The objective for the current study was to employ a targeted metabolomics approach to quantify ceramides, GlcCer, and lactosylceramides (LacCer) in plasma, and liver and skeletal muscle tissues collected from mid-lactation dairy cows supplemented with high-purity C16:0 for an extended duration, in comparison with a non-added fat control. We hypothesized that increasing dietary C16:0 would enhance ceramide synthesis, and ceramide availability would be positively 
associated with milk yield. Because insulin sensitivity improves and lipolysis is suppressed as lactation advances towards late gestation (McNamara and Hillers, 1986; Bell and Bauman, 1997), we expected control cows to exhibit a decline in circulating ceramide as lactation progressed.

\section{Materials and Methods}

\section{Experimental Design}

Experimental procedures were approved by the West Virginia University Institutional Animal Care and Use Committee at West Virginia University (Morgantown). Twenty multiparous, mid-lactation Holstein cows were housed in a free-stall barn and trained to access feed by using Calan gate feeders (American Calan, Inc., Norwood, NH) at the West Virginia University Animal Science Farm. Diets were mixed and offered twice daily. Access to feed was blocked from 0800 to $1000 \mathrm{~h}$ and 1600 to $1700 \mathrm{~h}$ to allow for orts collection and feeding. Cows were fed at $110 \%$ of expected intake, adjusted daily, and provided free access to water. Cows were milked twice daily at 0800 and $1800 \mathrm{~h}$. Milk yield was recorded daily.

All cows received a common TMR for $14 \mathrm{~d}$ prior to experimentation. During a $5 \mathrm{~d}$ covariate period, all cows received a common TMR without supplemental fat. Following the covariate period, cows were grouped by DIM and milk yield (136 $\pm 43 \mathrm{DIM}, 24.8 \pm 4.9 \mathrm{~kg}$ of milk/d), and offered a similar TMR consisting of no added fat (control; soyhull pellets; $n=10$ ) or supplemented with palmitic acid (PALM; Palmit 98; Global Agri-Trade, Long Beach, CA; $\mathrm{n}=10$ ) provided at $4 \%$ ration DM for $49 \mathrm{~d}$. Post-treatment, all cows were fed a common TMR for $14 \mathrm{~d}$. Diets provided were composed of sorghum silage and alfalfa haylage as the main forage components, and were 
formulated to meet nutrient recommendations (Table 5-1; NRC, 2001). Forages and concentrates were collected weekly, stored at $-20^{\circ} \mathrm{C}$, and composited at trial completion.

Blood collection $(10 \mathrm{~mL})$ by coccygeal venipuncture occurred on $\mathrm{d}-3,-2$, and 0 of the covariate period, and d 4, 6, 8, 14, 17, 21, 22, 24, 31, 38, 47, and 49 of the treatment period. In addition, blood was collected on d 52, 56, 62, and 63 , relative to the initiation of treatment to characterize the post-treatment period. Blood was kept on ice for 30 min until centrifugation at $3,400 \times g$ for 10 min. Following centrifugation, EDTA-preserved plasma was removed and stored at $-80^{\circ} \mathrm{C}$ until further analysis. Liver, skeletal muscle, and adipose tissue biopsies were performed on $\mathrm{d}-3$ and 47 relative to the start of treatment using aseptic techniques. For tissue biopsies, cows were anesthetized with xylazine $(0.04 \mathrm{~mL} / \mathrm{kg}$ of BW) delivered via the coccygeal vein. After the hair was clipped, biopsy sites were sanitized with iodine scrub and anesthetized with a $5 \mathrm{~mL}$ of lidocaine $\mathrm{HCl}$ (Vedco Inc., Saint Joseph, MO) delivered subcutaneously. For liver biopsies, the $11^{\text {th }}$ intercostal space was prepared by making a $0.5 \mathrm{~cm}$ incision through the skin, and a fabricated trocar was utilized to collect approximately $1 \mathrm{~g}$ of liver tissue (Hughes, 1962). Skeletal muscle biopsies were performed by making a $0.5 \mathrm{~cm}$ incision in the skin adjacent to the semitendinosus muscle. Approximately $500 \mathrm{mg}$ of muscle was collected using a Bard Magnum biopsy instrument mounted with a 12 gauge needle (Bard Biopsy Systems, Tempe, AZ). Following the collection of tissue, biopsy sites were stapled and sprayed with antiseptic, and Excenel RTU was delivered once by intramuscular injection $(0.68 \mathrm{~mL} / \mathrm{kg}$ of BW; Zoetis, Florham Park, NJ). Tissue for ceramide profiling was snap-frozen in liquid nitrogen and stored at $-80^{\circ} \mathrm{C}$. Tissue for PCR analysis was stabilized using RNAlater (Thermo Fisher Scientific Inc, Waltham, MA), refrigerated at $4^{\circ} \mathrm{C}$ during $24 \mathrm{~h}$, and stored at $-20^{\circ} \mathrm{C}$ until RNA isolation was performed. 


\section{Glucose Tolerance Tests}

Glucose tolerance tests (GTT) were performed on $\mathrm{d}-1,24$, and 49, relative to the start of treatment using previously described methods (Pires et al., 2007a; Schoenberg et al., 2012). Briefly, a jugular catheter was inserted $24 \mathrm{~h}$ prior to the GTT, and patency was maintained by flushing with heparinized saline every $12 \mathrm{~h}$. Coinciding with the removal of feed at $0800 \mathrm{~h}$, cows were intravenously infused with $300 \mathrm{mg}$ of glucose (dextrose, $50 \% \mathrm{wt} / \mathrm{vol}$ ) per $\mathrm{kg}$ of BW. Glucose infusion occurred within $8 \pm 0.88 \mathrm{~min}$, and was followed by a $10-\mathrm{mL}$ saline flush. Blood samples $(10 \mathrm{~mL})$ were collected at $-10,0,10,20,30,40,60,90,120,150$, and 180 min relative to initiation of glucose infusion. Blood was processed as described above. Feed was provided immediately following the completion of the GTT. The area under the curve for NEFA during GTT was calculated using the trapezoidal method as previously described by others (Pires et al., 2007a).

\section{Sample Analyses}

Individually composited feed ingredients were analyzed for NDF with heat-stable $\alpha$ amylase and sodium sulfite (Van Soest et al., 1991), CP (AOAC International, 2000; method 990.03), and starch (Hall, 2009) by Cumberland Valley Analytical Services Inc. (Cumberland, $\mathrm{MD})$.

To evaluate the relationship between ceramide and NEFA availability, we measured the concentrations of NEFA in plasma using an enzymatic method (HR series NEFA-HR; Wako Chemicals USA Inc., Richmond, VA). Spectrophotometric measurements were conducted using a SpectraMax Plus 384 Microplate Reader (Molecular Devices, Sunnyvale, CA). Intra- and interassay CV were 3.7 and $3.7 \%$ for plasma NEFA. 
Plasma ceramide extraction was conducted using previously established methods that employ a modified Bligh and Dyer procedure including ceramide C12:0 as an internal standard (Avanti Polar Lipids, Alabaster, AL; Haughey, et al., 2004; Bandaru et al., 2013; Rico et al., 2015). Briefly, plasma extracts were dried using a nitrogen evaporator and re-suspended in pure methanol for analysis. Sample extracts were handled using an autosampler (LEAP technologies Inc., Carrboro, NC) that introduced extracts into an HPLC (PerkinElmer, Boston, MA) with a C18 reverse-phase column (Phenomenex, Torrance, CA). Individual species of ceramide were separated by gradient elution and injected into an electrospray ion source coupled to a triple quadrupole mass spectrometer (API3000; AB Sciex Inc., Thornhill, Ontario, Canada; (Bandaru et al., 2007; Bandaru et al., 2011; Bandaru, 2013). Ion spray voltage was $5500 \mathrm{~V}$ at a temperature of $80^{\circ} \mathrm{C}$ with a nebulizer gas of $8 \mathrm{psi}$, curtain gas $8 \mathrm{psi}$, and collision gas 4 psi. Declustering potential was $80 \mathrm{~V}$, focusing potential $400 \mathrm{~V}$, entrance potential $10 \mathrm{~V}$, collision energy $30 \mathrm{~V}$, and collision cell exit potential $18 \mathrm{~V}$. Individual ceramide species were detected by multiple reaction monitoring. Six point calibration curves $(0.1$ to $750 \mathrm{ng} / \mathrm{ml})$ were constructed by plotting area under the curve for each calibration standard, C16:0-, C18:0-, C20:0-, C22:0-, and C24:0-ceramide, normalized to C12:0-ceramide. Sphingolipid concentrations were determined by curve fitting the identified ceramide species based on acyl-chain length. Quantification of spectral data was performed using Analyst 1.4.2 and MultiQuant software (AB Sciex Inc., Thornhill, Ontario, Canada).

To evaluate changes in hepatic gene expression, RNA isolation and real-time PCR were performed. RNA was extracted from frozen tissues using Trizol reagent (Invitrogen, Carlsbad, CA) according to manufacturer's instructions. Pellets of RNA were resuspended in RNase-free water and quantified at $260 \mathrm{~nm}$ using a spectrophotometer. Total RNA $(1 \mu \mathrm{g})$ was reverse 
transcribed into cDNA using the iScript cDNA synthesis kit (Bio-Rad, Hercules, CA) according to manufacturer's instructions. Realt-time PCR reactions were performed using the SYBR Green Master Mix qPCR kit (Bio Rad, Hercules, CA) and a CFX96 Real-time PCR machine (Bio-Rad, Hercules, CA). Quantification of gene transcripts for serine palmitoyltransferase 1 (SPT-1), ceramide synthases 1-6 (CerS1-6) and acid sphingomyelinase (SMase) was completed using genespecific primers (Supplemental Table 2). Ribosomal protein S9 (RPS9) was utilized as the endogenous control genes. Reaction conditions were as follows: 50 cycles of $95^{\circ} \mathrm{C}$ for $3 \mathrm{~min}, 95^{\circ} \mathrm{C}$ for $30 \mathrm{~s}, 60^{\circ} \mathrm{C}$ for $30 \mathrm{~s}$, and $72^{\circ} \mathrm{C}$ for $30 \mathrm{~s}$, followed by $95^{\circ} \mathrm{C}$ for $1 \mathrm{~min}, 60^{\circ} \mathrm{C}$ for $1 \mathrm{~min}$, and a temperature gradient from $60^{\circ} \mathrm{C}$ to $95^{\circ} \mathrm{C}$ in increments of $5^{\circ} \mathrm{C}$.

\section{Statistical Analysis}

All plasma data were analyzed as repeated measures over time relative to start of treatment under the MIXED procedure of SAS (version 9.3; SAS Institute Inc.). The statistical model included the random effect of cow, and the fixed effects of treatment and day relative to treatment start, and their interaction. The most appropriate covariance structure for the repeated measures analysis was selected for each variable after evaluating 5 different covariance structures (variance components, first-order autoregressive, heterogeneous first-order autoregressive, compound symmetry, and heterogeneous compound symmetry), and the structure with the smallest Akaike's information criterion coefficient was selected for analysis. Modeling of the covariance structure allowed the identification of patterns that best describe relationships between the repeated measures in the model. The method of Kenward-Rogers was used for calculation of denominator degrees of freedom. Normality of the residuals was evaluated with normal probability and box 
plots and homogeneity of variances with plots of residual versus predicted values. When necessary, data were transformed. Preplanned contrasts were used to evaluate the differences between treatments at each time point. Differences between treatments for tissue data were analyzed using the GLM procedure of SAS. Studentized residual values $>3.0$ or $<-3.0$ were considered outliers and removed from the analysis (typically 1 per response variable). Parametric Pearson correlations were performed in order to determine associations between plasma ceramides and milk yield, plasma NEFA and NEFA disappearance during glucose tolerance testing. All results are expressed as least squares means and their standard errors, unless stated otherwise. Significance was declared at $P<0.05$ and trends at $P<0.10$.

\section{Results and Discussion}

The development of insulin resistance is associated with the accumulation of liver-derived sphingolipid ceramide in plasma collected from hyperlipidemic type 2 diabetic animal models and dairy cows transitioning from gestation to lactation (Haus et al., 2009; Watt et al., 2012; Rico et al., 2015). Ceramides can be formed by de novo synthesis initiated by the condensation of palmitoyl-CoA via action of serine palmitoyltransferase I (Figure 5-1). Because palmitoyl-CoA is the principal substrate for de novo ceramide synthesis, we evaluated the effects of feeding an enriched C16:0 supplement to mid-lactation dairy cows for an extended duration, relative to a control without supplemental fat. The C16:0-enriched fat supplement was included at $3.9 \%$ of ration DM for PALM treatment, with soyhulls substituted in the control treatment (Table 5-1). A high dietary supply of C16:0 was used as a means to obtain a high fat diet (1.5-fold higher crude fat relative to control) that could induce elevated ceramide synthesis. Content of DM of NDF, ether 
extract, CP, starch, and net energy for lactation were closely matched between treatments (Table 5-1). To measure changes in circulating and tissue ceramide levels, we employed liquid chromatography coupled with mass spectrometry to perform a sphingolipidomic analysis of plasma ceramides and glycosylated ceramides, an approach described by Merrill et al. (2009) and utilized previously by our research group (Rico et al., 2015).

Detailed production and metabolic responses are described by Mathews et al. (J. Dairy Sci., Submitted). Briefly, PALM elevated energy intake, milk yield, ECM, milk fat and protein yield, and increased C16:0 incorporation in milk fat, relative to control. Estimated insulin sensitivity was lower during the first week of PALM treatment; however, glucose disposal following GTT was not modified during the duration of treatment. Although plasma NEFA declined with the progression of lactation, we did observe enhanced basal plasma NEFA and reduced glucose-stimulated NEFA disappearance by wk 7 of PALM treatment, suggesting localized adipose tissue insulin resistance and enhanced lipolysis in mid-lactation cows supplemented with C16:0.

The acyl chain diversity of sphingolipid ceramide is attributed to the action of six ceramide synthases (Figure 5-1; Park and Pewzner-Jung, 2013). Using a targeted metabolomics approach we quantified 21 ceramides, GlcCer, and LacCer in plasma, and liver and skeletal muscle tissue (Figure 5-2 and 5-3, and Figure 5-9). Comparable to diabetic humans (Haus et al., 2009) and our previous evaluation of insulin resistant peripartal dairy cows (Rico et al., 2015), C24:0-ceramide, C24:0-GlcCer, and C16:0-LacCer represented the major plasma and liver sphingolipids in each class. For instance, C24:0-ceramide represented approximately $70 \%$ of total ceramide measured in plasma. Lignoceroyl sphingosine (C24:0-ceramide) is a bioactive lipid mediator that can antagonize insulin-stimulated glucose uptake in mice and is elevated in type 2 diabetic humans 
(Boon et al., 2013; Haus et al., 2009). Because approximately $98 \%$ of plasma ceramides are found within liver-derived lipoprotein subfractions (i.e., high, low, and very low density lipoproteins; Lightle et al., 2003; Boon et al., 2013), we measured the concentrations of ceramides in the liver. As expected, our results demonstrate that C24:0-ceramide is the predominant hepatic ceramide. Recent evidence demonstrates that ceramides contained in low density lipoproteins are elevated in a type 2 diabetic state, promote macrophage inflammation, and reduce insulinstimulated glucose uptake in cultured myocytes (Boon et al., 2013). Boon and coworkers (2013) further demonstrated that ceramide is elevated in plasma membrane-enriched fractions of skeletal muscle obtained from mice infused with low density lipoprotein (LDL)-ceramide, a response accompanied by a reduction in Akt phosphorylation and insulin-stimulated glucose disposal. Although we did not measure adipose tissue ceramide levels, we did quantify the concentrations of ceramides and glycosylated ceramides in skeletal muscle. Our results reveal that C24:0ceramide is also the most abundant ceramide in skeletal muscle tissue, similar to our observed plasma profile, and to skeletal muscle measurements made in monogastrics (Haus et al., 2009; Boon et al., 2013). Relative to other ceramide subspecies, the biological importance of C24:0ceramide in plasma and tissues is unknown in ruminant animals.

Plasma ceramides are commonly elevated in insulin resistant humans experiencing hyperlipidemia, and mostly represent the hepatic ceramide output (Haus et al., 2009, Watt et al., 2012). Therefore, we compared the plasma ceramide and glycosylated ceramide profiles in coltrol and PALM cows (Figure 5-5). In agreement with our hypothesis, PALM increased the concentrations of total ceramide in plasma by $\mathrm{d} 7$ of treatment (Figure 5-6; $P<0.01$ ). The increase in circulating total ceramide was primarily due to C24:0-ceramide accumulation. Specifically, the concentration of C24:0-ceramide in plasma increased by $\mathrm{d} 7$ and remained elevated for the duration 
of PALM feeding (80\% increase on average; Figure 5-6; $P<0.001$ ). Likewise, concentrations of C16:1-, C22:0-, C22:1-, C24:1-, and C26:0-ceramide were greater in PALM-fed cows (Figure 56; $P<0.05)$. Following the removal of PALM, C16:1-, C22:0-, C22:1-, C24:0-, C24:1-, and C26:0-ceramide concentrations in plasma returned to control levels, although total ceramide tended to remain elevated $(P=0.09)$. We previously demonstrated that plasma concentrations of total ceramide and C24:0-ceramide increase concomitantly with NEFA mobilization and the development of insulin resistance as dairy cattle transition from gestation to lactation (Rico et al., 2015). Beyond peak milk production, insulin sensitivity improves and lipogenesis is favored as cows progress towards late lactation (McNamara and Hillers, 1986). In the present study, we observed a gradual decline in the availability of many circulating ceramides as control cows progressed through lactation, a response that was delayed with PALM. Interestingly, our observed decline in plasma ceramide occurred concurrently with a steady decrease in NEFA supply (Mathews et al., J. Dairy Sci. (Submitted)). In humans and rodents, increased availability of NEFA can promote insulin resistance by promoting ceramide accumulation in plasma and peripheral tissues (Adams et al., 2004; Holland et al., 2007; Watt et al., 2012). Our data supports a decrease in ceramide synthesis as mid-lactation cows advance towards late gestation, a response that is suppressed with continuous PALM feeding. Because ceramide synthesis is controlled by palmitoyl-CoA availability, the efficacy of other energy sources (i.e. other SFA or carbohydrates) to promote ceramide synthesis remains to be determined.

Glycosylated ceramides include GlcCer and LacCer, glycosphingolipids synthesized from ceramide and precursors for monosialodihexosylganglioside synthesis (GM3, a ganglioside; Figure 5-1). Similar to ceramide, GlcCer levels are elevated in plasma collected from insulin-resistant rodents (Chavez et al., 2014), and feeding a diet high in saturated fat can increase 
GlcCer availability (Aerts et al., 2007). In our experiment, PALM feeding increased total GlcCer and C24:0-GlcCer levels in plasma 32 and 33\% by wk 3 and 7, respectively (Figure 5-5 and 5-7; $P<0.001$ ), a response that was not observed following post-treatment. Similar increases C16:0-, C22:0-, C22:1-, and C24:1-GlcCer in plasma were also observed with PALM treatment (Figure 57); however PALM did not change total or individual LacCer levels in plasma (Figure 5-5 and Figure 5-9). Comparable to our observed changes in ceramide with time, we measured a gradual decline in the majority of GlcCer and LacCer as lactation progressed. For example, C24:0-GlcCer and C24:0-LacCer decreased 28 and $25 \%$ by wk 9 of experimentation, relative to the start of the study (Figure 5-7 and Figure 5-9; $P<0.05$ ). Similar to our previous work investigating ceramide availability in periparturient dairy cows (Rico et al., 2015), we observed a strong positive association between C24:0-ceramide and C24:0-GlCer across all sampled cows (Figure 5-10; $P<$ 0.001). These results continue to support a coordinated regulation of synthesis for these sphingolipids containing the C24:0 acyl moiety in dairy cows.

Evaluating the relationship between plasma and liver ceramides is a means to study sphingolipid metabolism because circulating ceramides are incorporated within lipoproteins (Lightle et al., 2003; Watt et al., 2012; Boon et al., 2013). Coinciding with the elevation in circulating ceramide with PALM treatment, we detected an increase in total ceramide, C22:0-, C22:1-, C24:0-, C24:1- and C26:0-ceramide within liver tissue collected at wk 7 (Figure 5-3; $P<$ 0.05). We recently proposed that hepatic ceramide synthesis is likely upregulated in dairy cows transitioning from gestation to lactation because of an increase in NEFA availability (Rico et al., 2015). The de novo synthesis of ceramide is controlled by SPT1 and CerS (Figure 5-1). Although feeding mid-lactation dairy cows C16:0 did not change the mRNA expression of SPT1 at d 47 of treatment (Figure 5-8); CerS2 and CerS5 mRNA were lower with PALM treatment. Because each 
isoform may control the synthesis of specific ceramides, we characterized the isoform distrubution in liver (Figure 5-8). While CerS6 was the predominant isoform, CerS2 and CerS5 mRNA were expressed equally. Interestingly, CerS2 and CerS6 can control the synthesis of abundant C24:0and C16:0-ceramide, respectively (Levy and Futerman, 2010). An alternative route for ceramides synthesis requires the activation of SMase to catalyze the hydrolysis of sphingomyelin, a response that is often accompanied by inflammation (Yang et al., 1993; Kolesnick and Golde, 1994; Peraldi et al., 1996). In our investigation, PALM decreased hepatic SMase mRNA expression (Figure 58). Considering that ceramide levels were elevated in liver and serum, reduced CerS and SMase mRNA expression may be a negative feedback mechanism to lower ceramide availability with extended PALM feeding. In addition to our observed changes with PALM feeding, our data also suggests that the decline in NEFA from mid to late lactation is accompanied by a decrease in hepatic ceramide synthesis and lipoprotein ceramide secretion, a response that is reversed during the acceleration of NEFA mobilization observed during early lactation (McNamara, 1991; Rico et al., 2015). Although the mechanisms involved in the transport of sphingolipid from the liver to the plasma are unknown in monogastrics and ruminants, current evidence suggests a role for microsomal triglyceride transfer protein (Iqbal et al., 2015).

We did not observe changes in skeletal muscle concentrations of total ceramide, GlcCer, or LacCer by wk 7 in PALM-fed cows (Figure 5-3). With the exception of C18:0-ceramide (23\% decrease; $P<0.05$ ), PALM feeding did not change skeletal muscle concentrations of individual ceramides or glycosylated ceramides (Figure 5-3). Intriguingly, infusing mice with LDL-ceramide did not increase total ceramide levels in skeletal muscle (Boon et al., 2013); however, LDLceramide induced insulin resistance was accompanied by plasma membrane ceramide accrual. In context of homeorhetic glucose partitioning toward the mammary gland, the ability of intracellular 
or extracellular ceramide to antagonize insulin action in skeletal muscle requires further investigation in dairy cows.

We have previously demonstrated in peripartal dairy cows that ceramide is positively correlated with plasma NEFA availability (Rico et al., 2015). To validate this relationship in our current investigation, we performed a Pearson's correlation analysis to identify significant associations between individual sphingolipid species and plasma NEFA levels. With the exception of C16:0-ceramide, all circulating ceramides, GlcCer, and LacCer were positively correlated with plasma NEFA concentrations $(P<0.05)$. The strongest correlations were observed for $\mathrm{C} 22: 0-$, C24:0-, and C26:0-ceramide $(0.41-0.53 ; P<0.001)$. Comparably, our previous work has demonstrated a remarkably similar relationship in peripartal dairy cows (Rico et al., 2015). Most notably, C16:0-ceramide availability is not linked with NEFA supply. Because we observed reduced glucose-stimulated NEFA disappearance by wk 7 of PALM treatment (Mathews et al., JDS (Submitted)), indicative of adipose tissue insulin resistance, we analyzed the correlative relationship between circulating ceramide supply and NEFA area under the curve (AUC) during a GTT (Table 5-2). Circulating ceramide and glycosylated ceramide were negatively correlated with NEFA AUC $(P<0.05)$. For instance, total ceramide and C24:0-ceramide were negatively correlated with NEFA AUC (-52 and -0.57 , respectively; $P<0.001)$. Although the effect of longchain plasma ceramide on lipolysis has not be characterized in dairy cows, culturing 3T3-L1 adipocytes with exogenous hydrophilic C2:0-ceramide can increase lipolysis by suppressing insulin-stimulated activation of cyclic nucleotide phosphodiesterase 3B (Mei et al., 2002). A limitation of our current work is that we have not established the origin of circulating NEFA. It is conceivable that under conditions of elevated lipid supply, such as those induced with elevated dietary fat supplementation, the rates of peripheral tissue FA uptake may be limited relative to the 
rate of delivery upon triacylglycerol hydrolysis by lipoprotein lipase in the blood capillaries. It is also possible, as we posit here, that circulating NEFA may also be originated from lipolysis in the adipose tissue, as a direct consequence of reduced insulin action.

Considering that ceramide induces insulin resistance in monogastrics (Summers et al., 1998), and insulin resistance in skeletal muscle and adipose tissue facilitates glucose partitioning to the mammary gland for milk synthesis (Bell, 1995; Spachmann et al., 2013), we hypothesized that milk yield would be associated with the availability of circulating ceramide. As expected, significant positive correlation coefficients between milk yields and the majority of ceramides and glycosylated ceramides were detected ranging from 0.25 to $0.51(P<0.05$; Table $5-2)$, with the strongest correlations observed for C22:0-, C24:0-, and C26:0-ceramide $(P<0.001)$. It should be noted that these strong relationships between circulating ceramides and milk yields were observed in mid-lactation cows experiencing positive energy balance. Whether a similar observation occurs in early lactation cows experiencing a greater magnitude of insulin resistance and lipolysis is uncertain.

The development of insulin resistance and lipolysis represent coordinated shifts in metabolism to support the onset of lactation, adaptations that fade with the progression of lactation. Although ceramides are known effectors of insulin resistance in monogastrics, the ability of ceramide to promote glucose partitioning toward the mammary gland is unknown. In our study, we were able to demonstrate that PALM feeding was able to delay the decline in ceramide availability with the advancement of lactation. Future research we will need to determine whether ceramide mediates insulin resistance in dairy cows. If it is established that ceramide can antagonize insulin action, then promoting C16:0 intake in dairy cattle may be a nutritional approach for cows to stimulate a homeorhetic response beyond milk yield. We recognize that insulin resistance and 
NEFA mobilization during early lactation is accompanied by ceramide accrual (Rico et al., 2015); therefore, we cautiously contemplate whether C16:0 supplementation could augment ceramide supply and insulin resistance during early lactation, and inadvertently challenge metabolic health. These uncertainties will need to be addressed with further investigation.

\section{Conclusions}

Our targeted metabolomics approach has revealed that long-term feeding of C16:0 to midlactation cows increased hepatic synthesis and plasma accrual of ceramide. The ability of increased C16:0 intake to augment ceramide supply delayed the decline in ceramide supply observed with the progression of lactation. We also conclude that an increase in ceramide supply is associated with elevated basal NEFA, reduced adipose tissue NEFA disappearance to a glucose challenge, and milk yield. In support of our previous work (Rico et al., 2015), our data suggests that ceramides may be intrinsically involved in the homeorhetic adaptation to lactation. To confirm this possibility, further studies should investigate whether ceramide mediates the development of systemic or localized insulin resistance in dairy cattle, and how these relationships are influenced by stage of lactation. Specific attention should be focused on highly abundant C24:0-ceramide. 
Table 5-1. Ingredients and nutrient composition (\% of DM unless otherwise noted) of experimental diets supplemented with palmitic acid (PALM; C16:0) and control (no added fat).

\begin{tabular}{lcc}
\hline & \multicolumn{2}{c}{ Treatment } \\
\cline { 2 - 3 } Item & Control & PALM \\
\hline Ingredient (\% DM) & 30.3 & 30.1 \\
$\quad$ Sorghum sudangrass & 29.1 & 28.9 \\
Ground corn & 15.7 & 15.5 \\
Alfalfa haylage & 11.4 & 11.3 \\
Soybean meal & 6.6 & 3.6 \\
Soyhulls & 2.9 & 2.8 \\
Beet pulp pellets & 0.63 & 0.60 \\
Vitamin and mineral premix ${ }^{1}$ & 1.60 & 1.60 \\
Protein blend & \\
Sodium bicarbonate & 0.97 & 0.95 \\
Zeolite & 0.48 & 0.46 \\
Limestone & 0.32 & 0.29 \\
C16:0-enriched fat supplement & 3 \\
Nutrient composition & 0.0 & 3.9 \\
DM, \% & & \\
CP, \% DM & 59.6 & 59.8 \\
NDF, \% DM & 17.8 & 17.3 \\
Forage NDF, \% DM & 33.6 & 31.6 \\
Starch, \% DM & 24.8 & 24.8 \\
Ether extract, \% DM & 21.7 & 21.6 \\
Ash, \% DM & 2.8 & 6.7 \\
NE, Mcal/kg DM & 12.6 & 13.1 \\
\hline Vitamin-mineral mix contained & 1.4 & 1.5 \\
\hline
\end{tabular}

${ }^{1}$ Vitamin-mineral mix contained $14 \% \mathrm{Ca}, 9.5 \% \mathrm{P}, 6.3 \% \mathrm{Mg}, 0.13 \% \mathrm{~K}, 0.78 \% \mathrm{~S}, 4 \% \mathrm{Na}, 17.6 \% \mathrm{C}, 4282$ ppm Fe, 3000 ppm Zn, 590 ppm Cu, 1600 ppm Mn, 62 ppm Se, 53 ppm Co, and 31 ppm I, as well as 507 kIU Vit. A, 69 kIU Vit. D, and 1997.6 kIU Vit. E.

${ }^{2}$ Protein blend contained $30.1 \%$ canola meal solvent, $1.05 \%$ smartamine (Adisseo, Antony, France), $11.1 \%$ corn gluten meal $(60 \%), 45.7 \%$ soy plus, and $12.1 \%$ blood meal.

${ }^{3}$ Palmitic acid supplement contained 98\% palmitic acid (Palmit 98; Global Agri-Trade, Long Beach, CA, USA). 


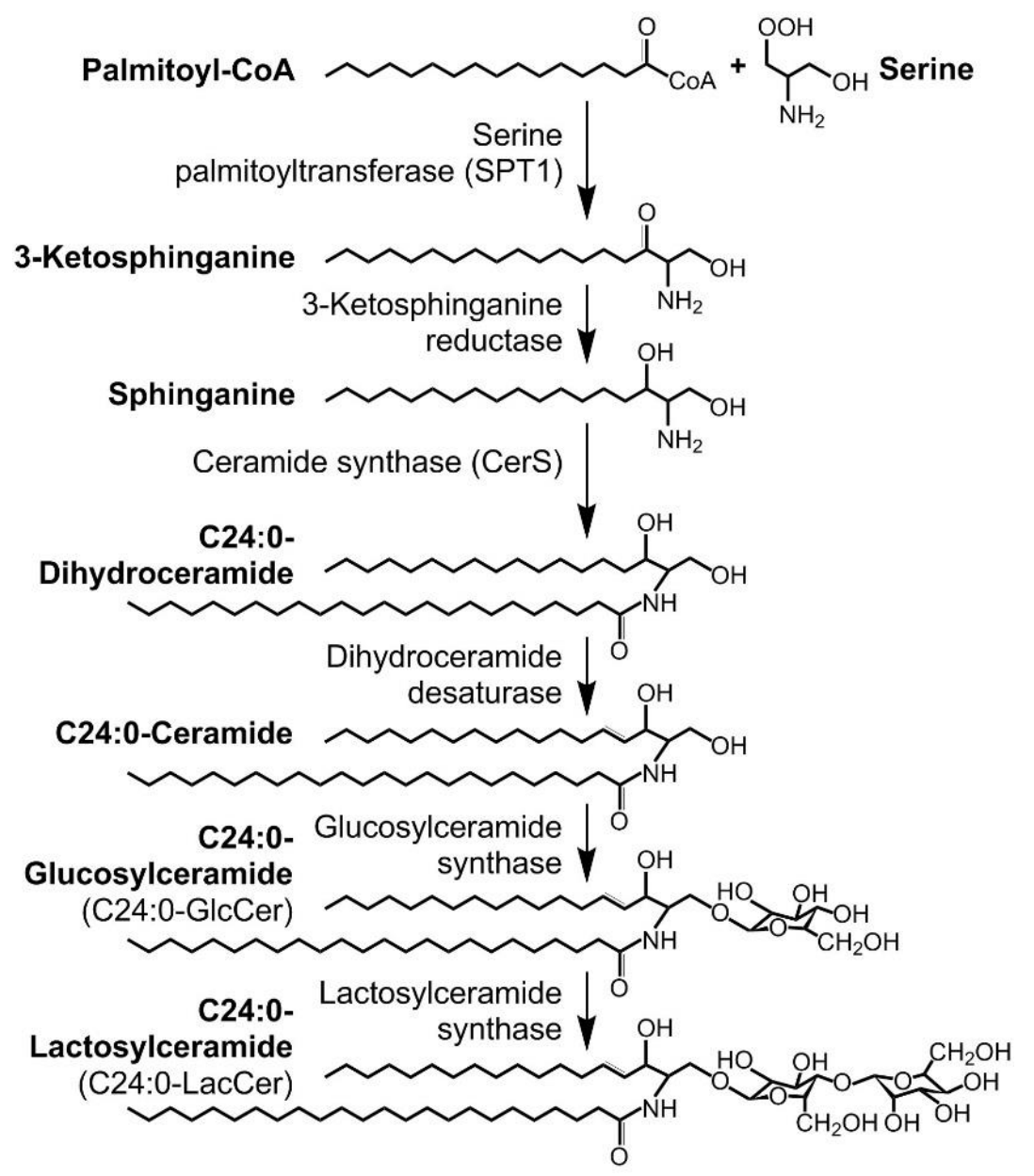

Figure 5-1. De novo ceramide synthesis.

Ceramide de novo synthesis initiated by the condensation of palmitoyl-CoA and serine to form 3ketosphinganine. For simplicity, only C24:0-linked sphingolipids and glycosphingolipids are illustrated. Monohexosylceramides (GlcCer) consist of a single sugar residue, either glucose or galactose (galactosylceramide is not shown). 


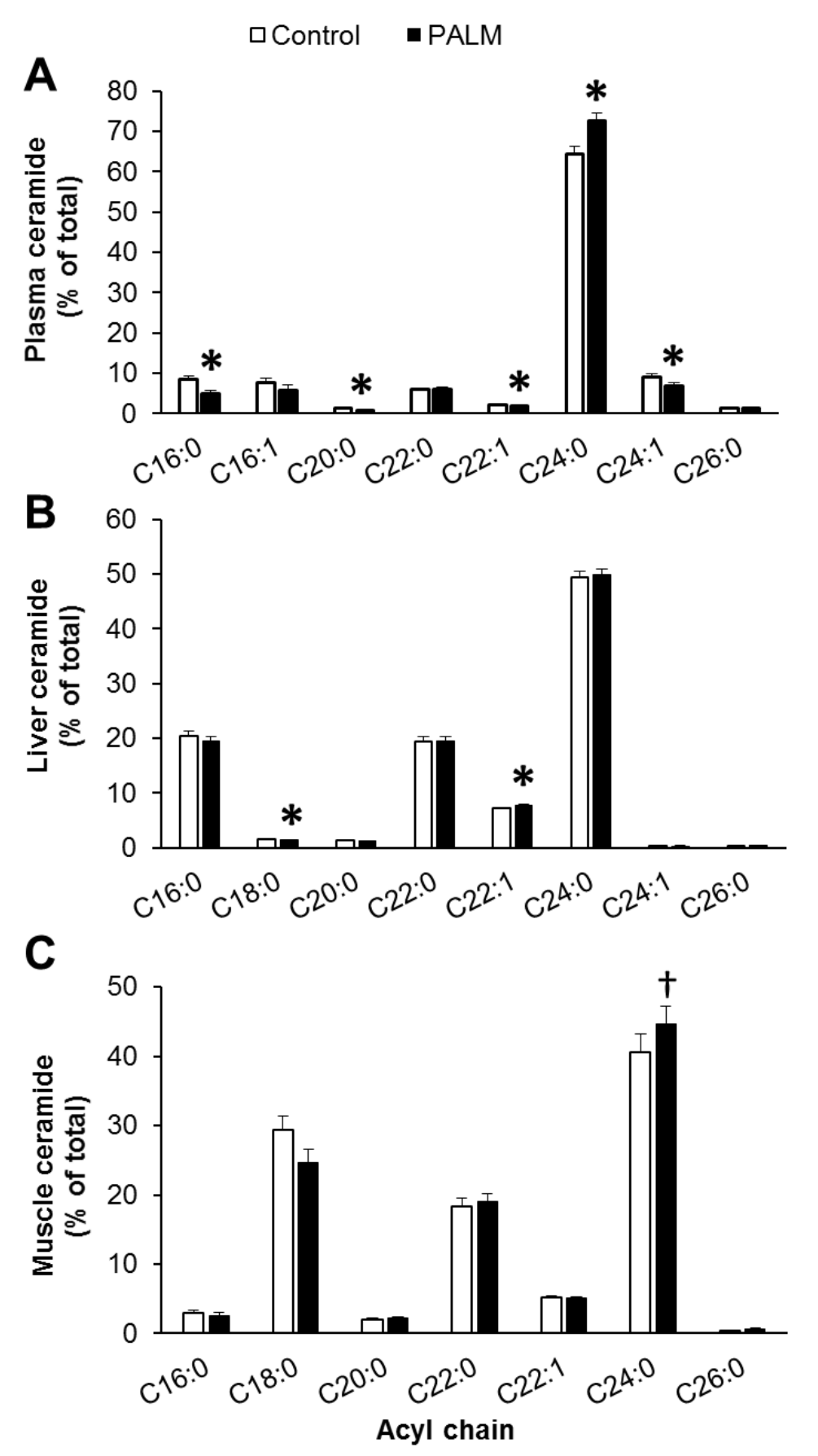

Figure 5-2. C24:0-ceramide is the most abundant ceramide in plasma, and liver and skeletal muscle tissue of lactating dairy cows fed control or palmitic acid-supplemented diets (PALM).

Ceramide profile of (A) plasma, and (B) liver and (C) skeletal muscle tissue at d 47 of treatment. C16:0-dihydro-lactosylceramide is not included. Data are represented as least squares means and their standard errors. ${ }^{*}, P<0.05 ; \uparrow, P<0.10$. 


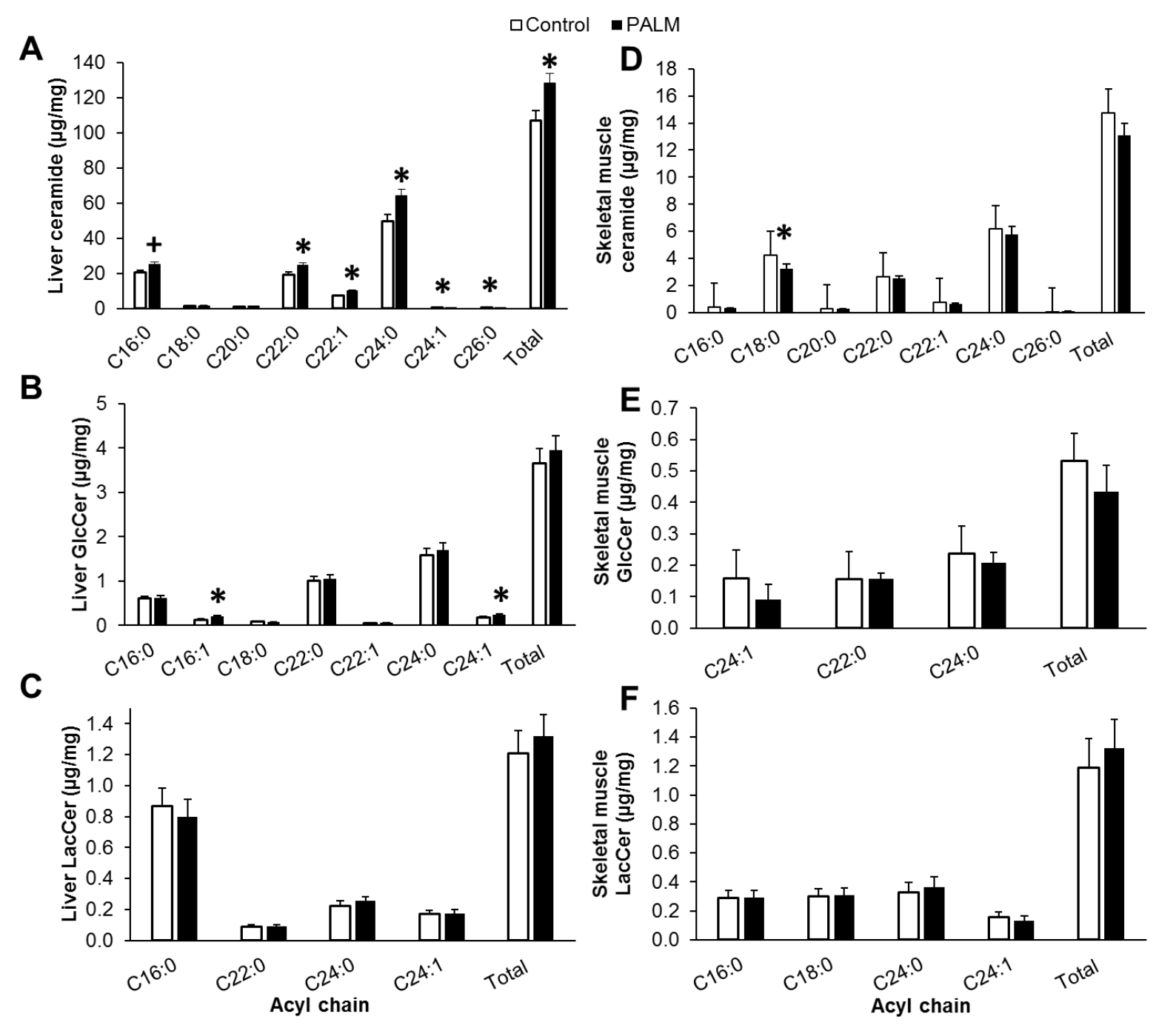

Figure 5-3. Palmitic acid-feeding (PALM) increases hepatic ceramide concentrations (ng/mg).

Liver concentration of (A) ceramide, (B) monohexosylceramide (GlcCer), and (C) lactosyceramide (LacCer), and skeletal muscle concentration of (D) ceramide, (E) GlcCer, and (F) LacCer in control and PALM-fed cows at d 47 of treatment. Data are represented as least squares means and their standard errors. ${ }^{*}, P<0.05$. 


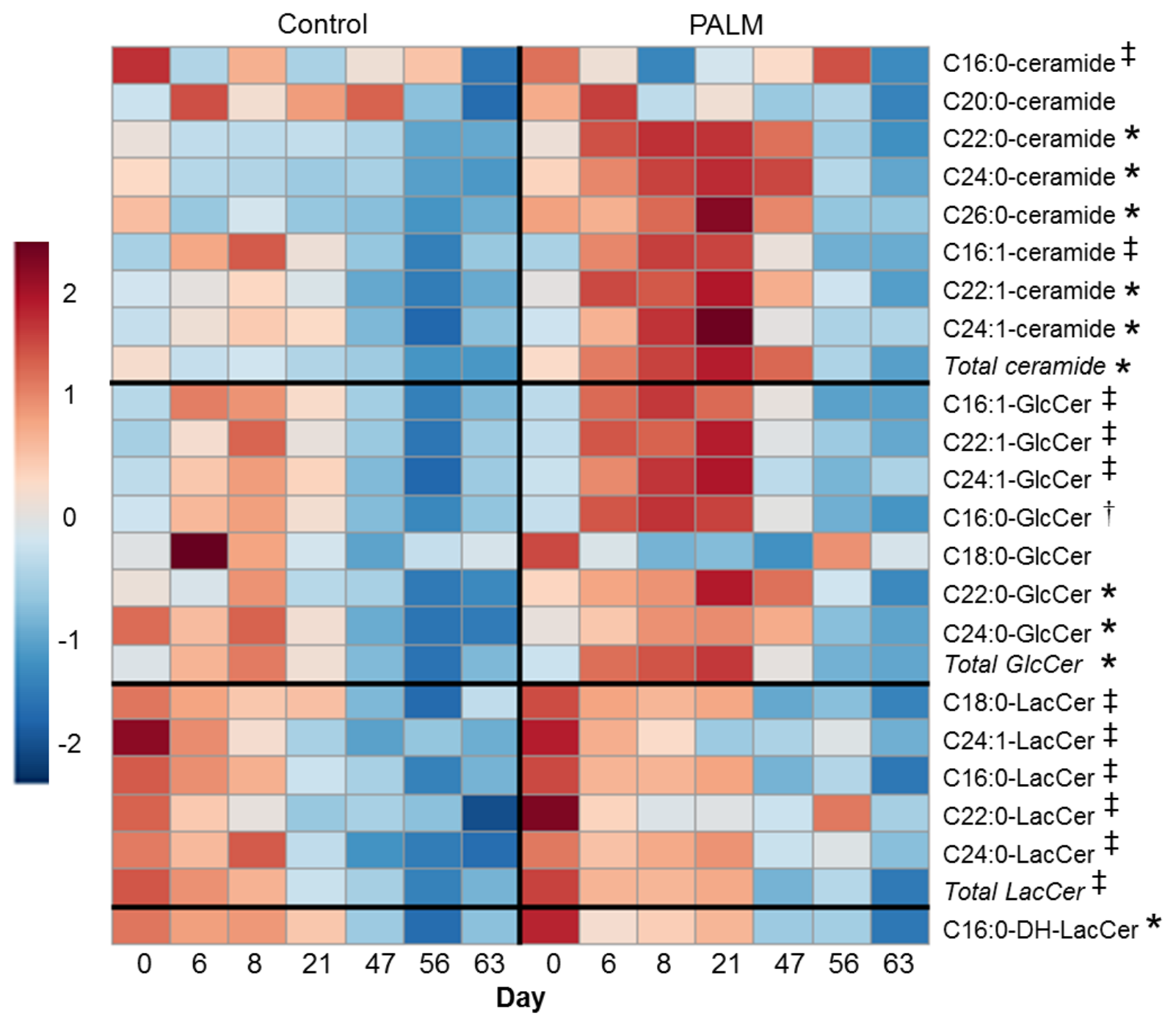

Figure 5-4. Plasma ceramides and monohexosylceramides (GlcCer) are elevated in palmitic acidfed (PALM) cows, relative to control cows.

For visualization purposes, the heat map represents relative concentrations as high (red) or low (blue). The heat map was generated using MetaboAnalyst 3.0 (Xia et al., 2012). Data are represented as least squares means and their standard errors. $\ddagger$, main effect of day relative to treatment start, $P<0.05$; *, Treatment $\times$ Day interaction, $P<0.05 ; \uparrow, P<0.10$. Abbreviation: LacCer: Lactosylceramide; DH: dihydro. 


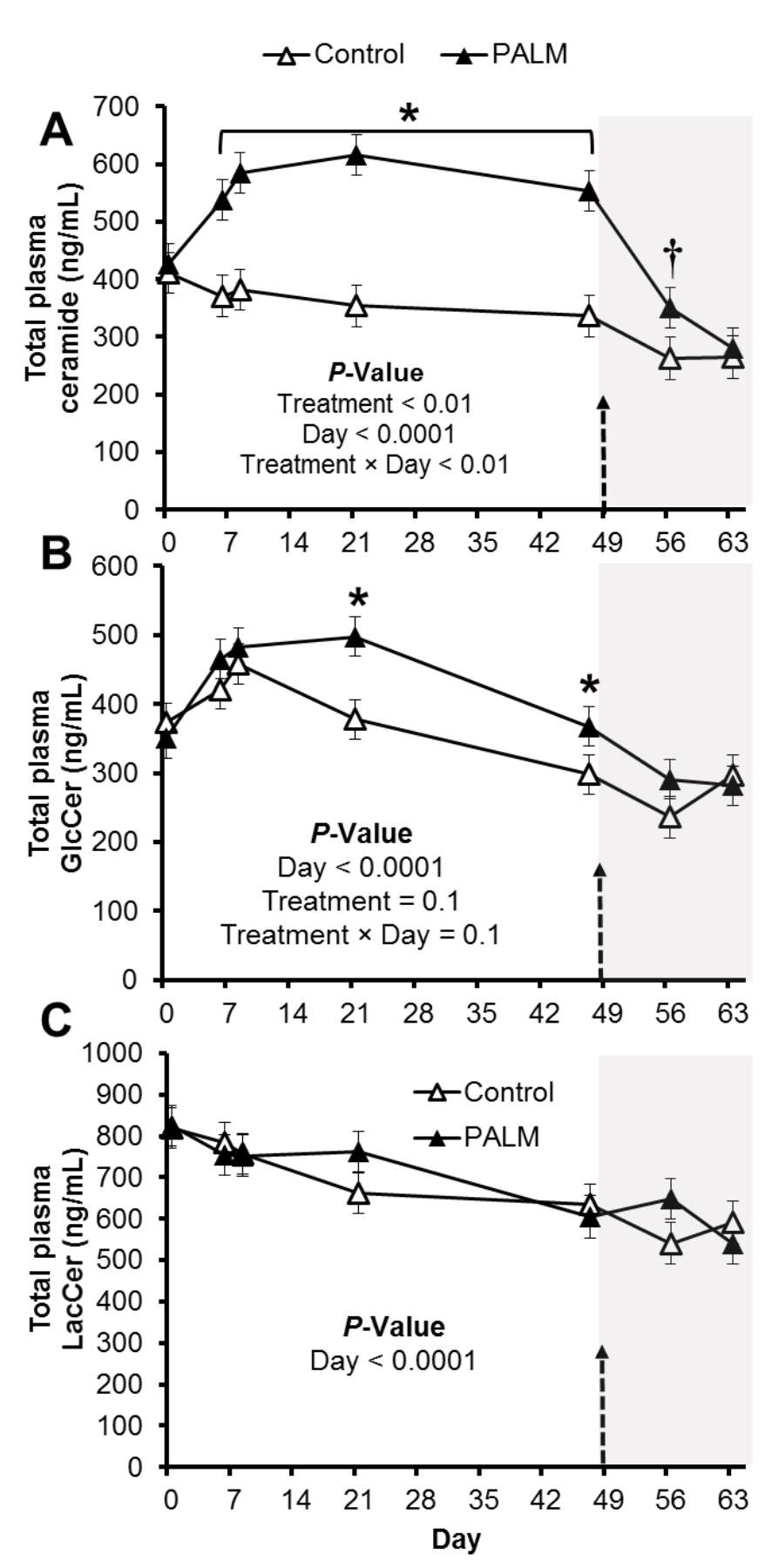

Figure 5-5. Palmitic acid-feeding (PALM) increases total ceramide and monohexosylceramide (GlcCer) concentrations $(\mathrm{ng} / \mathrm{mL})$ in plasma.

Plasma concentration of total (A) ceramide, (B) GlcCer, and (C) lactosyceramide (LacCer) in control and PALM-fed cows. Dotted arrows denote the end of treatment period. Data are represented as least squares means and their standard errors. ${ }^{*}, P<0.05 ; \uparrow, P<0.10$. 

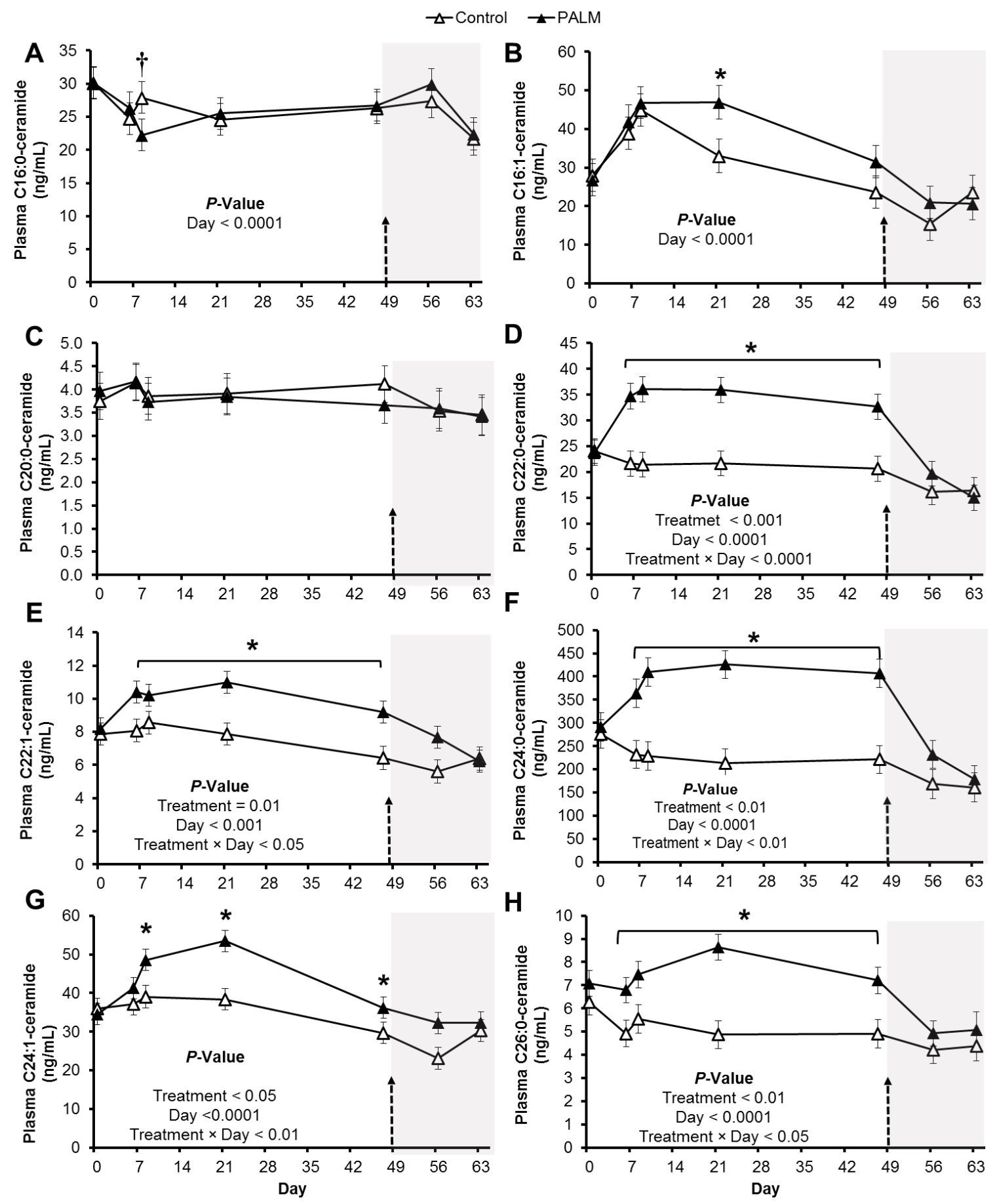

Figure 5-6. Plasma concentration (ng/mL) of C24:0-ceramide is elevated in palmitic acid-fed (PALM) cows, relative to control cows.

Plasma concentration of (A) C16:0-, (B) C16:1-, (C) C20:0-, (D) C22:0-, (E) C22:1-, (F) C24:0-, (G) C24:1-, and (H) C26:0-ceramide. Dotted arrows denote the end of treatment period. Data are represented as least squares means and their standard errors. ${ }^{*}, P<0.05 ; \dagger, P<0.10$. 

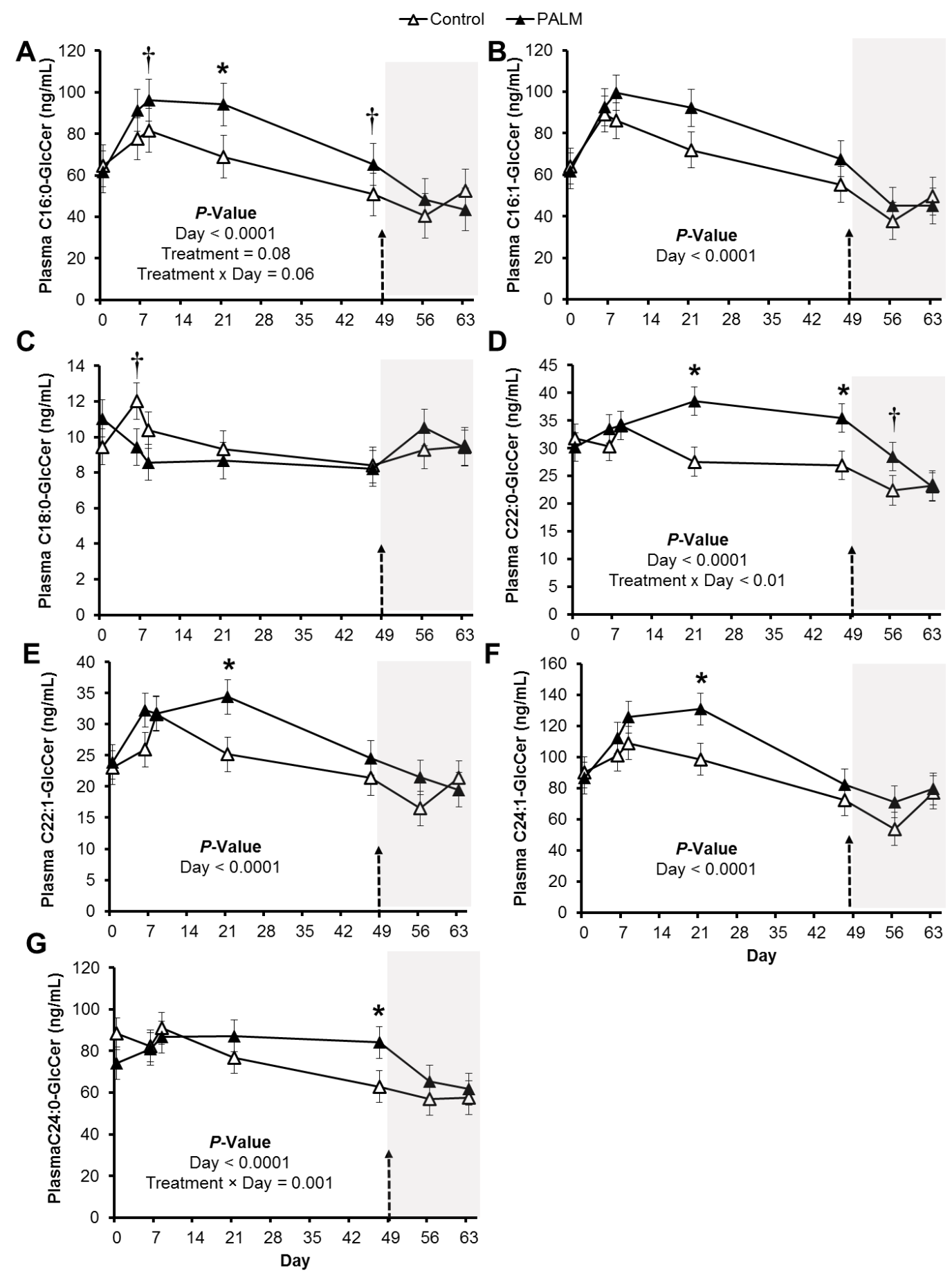

Figure 5-7. Plasma concentration (ng/mL) of C24:0-monohexosylceramide (GlcCer) is elevated in palmitic acid-fed (PALM) cows, relative to control cows.

Plasma concentration of (A) C16:0-, (B) C16:1-, (C) C18:0-, (D) C22:0-, (E) C22:1-, (F) C24:0and (G) C24:1-GlcCer. Dotted arrows denote the end of treatment period. Data are represented as least squares means and their standard errors. ${ }^{*}, P<0.05 ; \dagger, P<0.10$. 

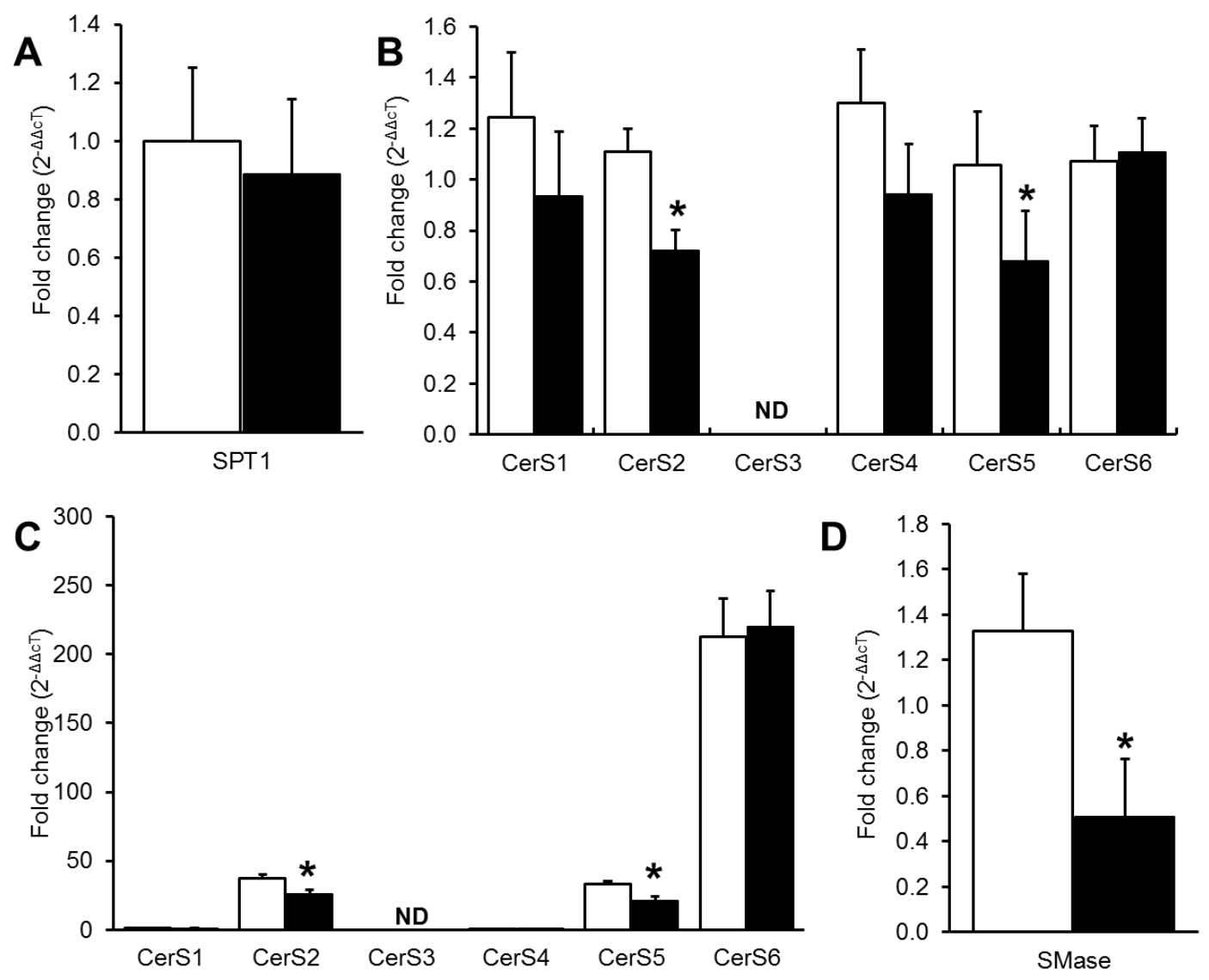

Figure 5-8. Hepatic expression of ceramides synthase (CerS) and acid sphingomyelinase (SMase) is lower in cows fed palmitic acid (PALM) for an extended duration, relative to control cows.

Effect of PALM on the hepatic mRNA expression of (A) SPT1 and (B) CerS, relative to control cows. (C) Hepatic distribution of CerS1-6 mRNA. (D) Change in SMase mRNA expression. Ribosomal protein S9 was used as the endogenous control gene and control CerS1 was used as the calibrator for making relative comparisons across isoforms. Data are represented as least squares means and their standard error bars calculated using the $2^{-\Delta \Delta C}$. An asterisk indicates a significant difference in $\Delta C_{\mathrm{t}}$ from control. ND $=$ Not detected. $*, P<0.05$. 
Table 5-2. Plasma ceramides are positively correlated with circulating NEFA and milk yield, and negatively associated with area under the curve of NEFA during a glucose challenge in dairy cows during mid-lactation.

\begin{tabular}{|c|c|c|c|c|c|c|}
\hline & \multicolumn{2}{|c|}{ Milk yield } & \multicolumn{2}{|c|}{ NEFA } & \multicolumn{2}{|c|}{ AUC 180} \\
\hline & $r$ & $P$-value & $r$ & $P$-value & $r$ & $P$-value \\
\hline \multicolumn{7}{|l|}{ Ceramide } \\
\hline C16:0 & 0.19 & $<0.10$ & 0.10 & 0.26 & -0.19 & 0.16 \\
\hline C16:1 & -0.01 & 0.91 & 0.23 & $<0.01$ & -0.05 & 0.72 \\
\hline C20:0 & 0.37 & $<0.01$ & 0.24 & $<0.01$ & -0.31 & $<0.05$ \\
\hline C22:0 & 0.43 & $<0.001$ & 0.53 & $<0.001$ & -0.39 & $<0.01$ \\
\hline C22:1 & 0.14 & 0.24 & 0.41 & $<0.001$ & -0.34 & $<0.01$ \\
\hline C24:0 & 0.44 & $<0.001$ & 0.52 & $<0.001$ & -0.57 & $<0.001$ \\
\hline C24:1 & 0.15 & 0.18 & 0.38 & $<0.001$ & -0.19 & 0.14 \\
\hline C26:0 & 0.51 & $<0.001$ & 0.41 & $<0.001$ & -0.55 & $<0.001$ \\
\hline Total & 0.39 & $<0.001$ & 0.53 & $<0.001$ & -0.51 & $<0.001$ \\
\hline \multicolumn{7}{|l|}{ GlcCer } \\
\hline C16:0 & 0.00 & 0.99 & 0.35 & $<0.001$ & -0.17 & 0.19 \\
\hline C16:1 & -0.08 & 0.49 & 0.30 & 0.001 & -0.08 & 0.57 \\
\hline C18:0 & -0.22 & $<0.10$ & -0.23 & $<0.01$ & 0.13 & 0.34 \\
\hline C22:0 & 0.42 & $<0.001$ & 0.35 & $<0.001$ & -0.53 & $<0.001$ \\
\hline C22:1 & -0.11 & 0.33 & 0.19 & $<0.05$ & -0.03 & 0.80 \\
\hline C24:0 & 0.35 & $<0.01$ & 0.29 & $<0.001$ & -0.39 & $<0.01$ \\
\hline C24:1 & -0.09 & 0.44 & 0.24 & $<0.01$ & 0.01 & 0.94 \\
\hline Total & 0.08 & 0.46 & 0.34 & $<0.001$ & -0.24 & $<0.10$ \\
\hline \multicolumn{7}{|l|}{ LacCer } \\
\hline C16:0 & 0.25 & $<0.05$ & 0.31 & $<0.001$ & -0.46 & $<0.001$ \\
\hline C18:1 & 0.06 & 0.62 & 0.36 & $<0.001$ & -0.44 & $<0.001$ \\
\hline C22:0 & 0.31 & $<0.01$ & 0.40 & $<0.001$ & -0.55 & $<0.001$ \\
\hline C24:0 & 0.36 & $<0.01$ & 0.45 & $<0.001$ & -0.59 & $<0.001$ \\
\hline C24:1 & -0.10 & 0.38 & 0.41 & $<0.001$ & -0.41 & $<0.01$ \\
\hline Total & 0.24 & $<0.05$ & 0.33 & $<0.001$ & -0.47 & $<0.01$ \\
\hline $\begin{array}{l}\text { C16:0-DH- } \\
\text { LacCer }\end{array}$ & 014 & 0.23 & 032 & $<0.001$ & -0.28 & $<0.05$ \\
\hline
\end{tabular}

Pearson's correlations $(r)$ of circulating ceramides to plasma NEFA, milk yield, and area under curve (AUC) for $180 \mathrm{~min}$ following a glucose challenge. DH, dihydro; GlcCer, monohexosylceramide; LacCer, lactosylceramide. 

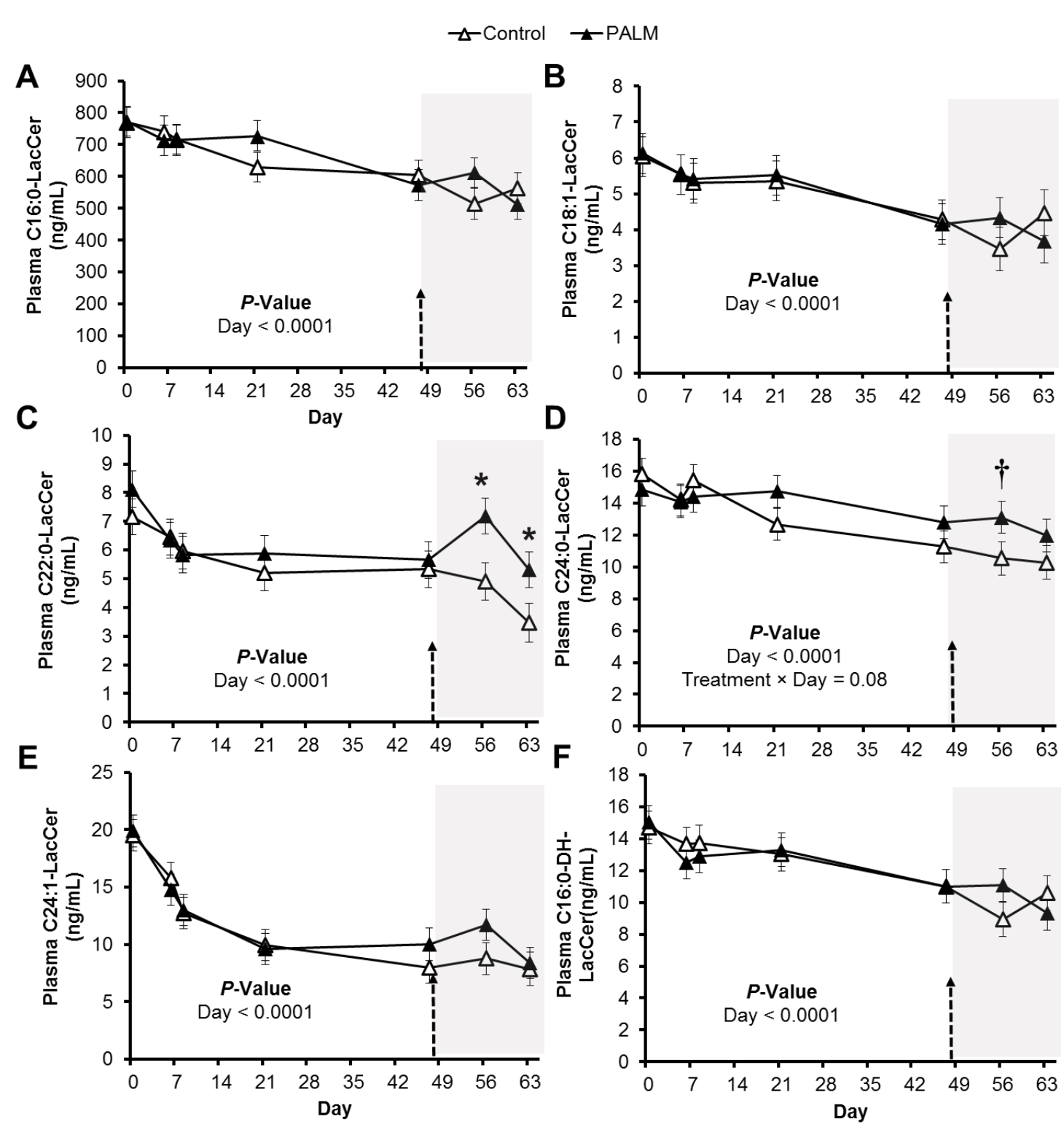

Figure 5-9. Supplemental 5-1. Plasma concentration $(\mathrm{ng} / \mathrm{mL})$ of lactosylceramide (LacCer) in control and palmitic acid-fed (PALM) cows.

Plasma concentration of (A) C16:0-, (B) C18:1-, (C) C22:0-, (D) C24:0-, (E) C24:1-, and (F) C16:0-dihydro-LacCer. Dotted arrows denote the end of treatment period. Data are represented as least squares means and their standard errors. ${ }^{*}, P<0.05 ; \dagger, P<0.10$. 


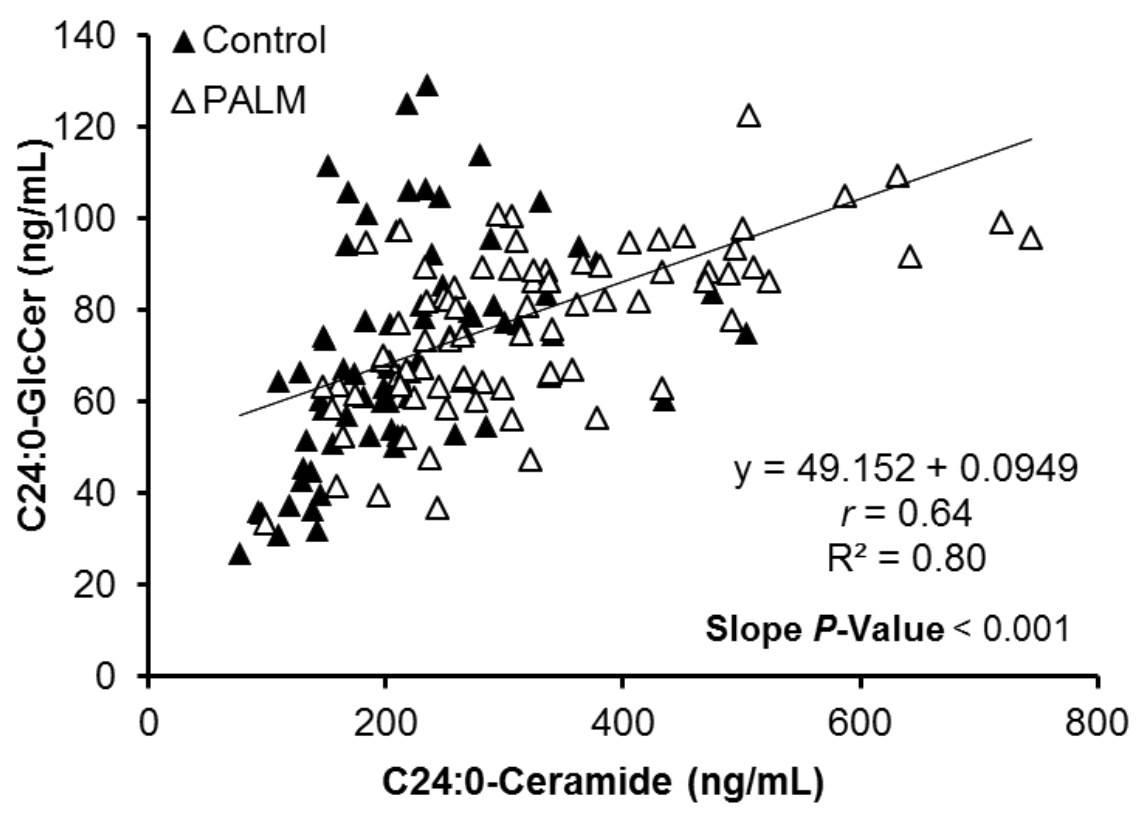

Figure 5-10. Supplemental 5-2. Plasma C24:0-monohexosylceramide (GlcCer) is positively associated with C24:0-ceramide in lactating dairy cows.

Random regression analysis between plasma concentrations of C24:0-GlcCer and C24:0-ceramide in lactating dairy cows fed control or palmitic acid-supplemented (PALM) diets. Coefficients of determination $\left(\mathrm{R}^{2}\right)$ represent the association between observed and model predicted values when accounting for the random effect of cow. Data represent measurements for d 0, 23 and 49, relative to start of treatment. 
Table 5-3. Supplemental 5-1. Nucleotide and PCR product specifications

\begin{tabular}{|c|c|c|c|c|}
\hline Gene $^{1}$ & Sequence $5^{\prime}-3^{\prime}$ & $\begin{array}{l}\text { Gen Bank } \\
\text { accession no. }\end{array}$ & $\begin{array}{l}\operatorname{Tm} \\
\left(C^{\circ}\right)\end{array}$ & $\begin{array}{l}\text { Product } \\
\text { length } \\
\text { (bp) }\end{array}$ \\
\hline $18 S-r R N A^{2}$ & $\begin{array}{l}\text { Fwd: TGCATGGCCGTTCTTAGTTG } \\
\text { Rv: AGAGTCTCGTTCGTTATCGGAATT }\end{array}$ & DQ222453 & 55 & 65 \\
\hline RPS9 & $\begin{array}{l}\text { Fwd : CCTCGACCAAGAGCTGAAG } \\
\text { Rv : CCTCCAGACCTCACGTTTGTTC }\end{array}$ & DT860044 & 56 & 62 \\
\hline CerS1 & $\begin{array}{l}\text { Fwd : CCCTCCGTTTTCTATGACTGG } \\
\text { Rv : AAGCATCCAGGTACAGTGTGG }\end{array}$ & NM_001083514 & 60 & 120 \\
\hline CerS2 & $\begin{array}{l}\text { Fwd : CCCTAACTTGGACCAGAAACC } \\
\text { Rv : CTGTCTGAGCAGTCCACATTG }\end{array}$ & NM_001034667 & 60 & 147 \\
\hline CerS3 & $\begin{array}{l}\text { Fwd: GGTTTAGGAGACGGCAGAATC } \\
\text { Rv e: CCCACAGGTCATATACCCAAG }\end{array}$ & NM_001099389 & 60 & 144 \\
\hline CerS4 & $\begin{array}{l}\text { Fwd: GGGAAAACTACCCACATCAGC } \\
\text { Rv : ACCTGCTCCTTGAAGTCCTTG }\end{array}$ & NM_001015520 & 60 & 129 \\
\hline CerS5 & $\begin{array}{l}\text { Fwd: CTGAACACGACCCTCTTTGAG } \\
\text { Rv: GACCAGATGACATGCAGAACC }\end{array}$ & NM_001102132 & 60 & 107 \\
\hline Cers6 & $\begin{array}{l}\text { Fwd: AGGCTTGAACTGCTTCTGGTC } \\
\text { Rv: GGTTCTTTCCTGGAGGTTCTG }\end{array}$ & NM_001193132 & 60 & 139 \\
\hline SPT1 & $\begin{array}{l}\text { Fwd : GAAGAGACCACTGGGTGTCG } \\
\text { Rv : ACCACAACCCTAATGCTCGG }\end{array}$ & NM_001034749 & 60 & 149 \\
\hline
\end{tabular}

${ }^{1} 18 \mathrm{~s}$ rRNA = 18s ribosomal RNA; RPS9 = ribosomal protein S9; CerS1-CerS6 = ceramide synthases 1-6; SPT1 $=$ serine palmitoyltransferase subunit 1 . All genes are specific to Bos Taurus .

${ }^{2}$ Sequence from Janovick-Guretzky et al., 2007. 


\section{Chapter 6}

\section{GENERAL CONCLUSIONS}

With the experiments presented in this dissertation, we have evaluated the associations between fatty acid availability, ceramide synthesis, and insulin action in dairy cows. Using mass spectrometry, we have been able to comprehensively profile plasma and tissue sphingolipids during pregnancy and lactation. This approach allowed us to identify meaningful patterns by which the sphingolipidome is remodeled in association with parturition, the onset of lactation, as well as with body fat accumulation. We established that sphingomyelin is the most abundant sphingolipid in plasma, followed by ceramides and glycosylated ceramides. We conclude that both the availability of circulating fatty acids and the accumulation of lipids in the liver can positively influence the synthesis of ceramides and increase their accumulation in liver, plasma, and skeletal muscle. We demonstrated that ceramide accumulation can be modulated by means of dietary manipulation, and that increased availability of the saturated fatty acid palmitate can effectively enhance ceramide synthesis. Moreover, our data suggest that circulating NEFA and sphingomyelin contribute to ceramide synthesis during peripartum and established lactation, however, the relative contributions from each pathway cannot be established from our results.

As expected, plasma ceramides were consistently inversely associated with insulin sensitivity. The negative associations between ceramides and insulin sensitivity in lean and overweight cows support the potential involvement of ceramide both in the homeorhetic development of insulin resistance during peripartum, as well as in its pathological manifestation, observed in hyperlipidemic overweight cows undergoing accelerated body weight loss and hepatic fat accumulation. Moreover, our observations are in line with a role for ceramides in provoking 
adipose tissue-specific insulin resistance, as manifested by reduced rates of NEFA disappearance in animal with elevated plasma ceramides.

Although these experiments provide useful information regarding the association between ceramides, fatty acid availability and insulin resistance, future investigations will need to validate our findings by directly evaluating the ability to ceramides to cause insulin resistance in diary cows. Considering the large number of sphingolipids associated with NEFA, insulin sensitivity, and milk production, future research should evaluate the potential use of ceramides and sphingomyelins as predictors of metabolic risk and milk production. The manipulation of ceramide synthesis and the identification of mechanism of insulin resistance constitute promising areas with the potential to modulate productive responses, improve insulin sensitivity and to improve cow health. 


\section{REFERENCES}

Adams, J.M., T. Pratipanawatr, R. Berria, E. Wang, R.A. DeFronzo, M.C.Sullards, and L.J. Mandarino. 2004. Ceramide content is increased in skeletal muscle from obese insulinresistant humans. Diabetes. 53:25-31.

Aerts, J.M., R. Ottenhoff, A.S. Powlson, A. Grefhorst, M. van Eijk, P.F. Dubbelhuis, J. Aten, F. Kuipers, M.J. Serlie, T. Wennekes, J.K Sethi, S. O'Rahilly, H.S. Overkleeft. 2007. Pharmacological inhibition of glucosylceramide synthase enhances insulin sensitivity. Diabetes. 56:1341-1349.

Ali, A. K. A., and G. E. Shook. 1980. An optimum transformation for somatic cell concentration in milk. J. Dairy Sci. 63:487-490.

Annison, E. F. and R. R White. 1961. White Glucose utilization in sheep. Biochem J. 80: 162169.

AOAC. 1990. Official Methods of Analysis. Vol. 2. 15th ed. Assoc. Off. Anal. Chem., Arlington, VA. AOAC International. 1995. Official Methods of Analysis. 16th ed. AOAC International, Arlington, VA.

Aschenbach, J.R., N.B. Kristensen, S.S. Donkin, H.M. Hammon, and G.B. Penner. 2010. Gluconeogenesis in dairy cows: the secret of making sweet milk from sour dough. IUBMB Life. 62:869-77.

Bandaru, V. V., J. C. McArthur, N. Sacktor, R. G. Cutler, E. L. Knapp, M. P. Mattson, and N. J. Haughey. 2007. Associative and predictive biomarkers of dementia in HIV-1-infected patients. Neurology 68:1481-1487.

Bandaru, V. V., M. M. Mielke, N. Sacktor, J. C. McArthur, I. Grant, S. Letendre, L. Chang, V. Wojna, C. Pardo, P. Calabresi, S. Munsaka, and N. J. Haughey. 2013. A lipid storage-like disorder contributes to cognitive decline in HIV-infected subjects. Neurology 81:1492-1499.

Bandaru, V. V., N. Patel, O. Ewaleifoh, and N. J. Haughey. 2011. A failure to normalize biochemical and metabolic insults during morphine withdrawal disrupts synaptic repair in mice transgenic for HIV-gp120. J. Neuroimmune Pharmacol. 6:640-649.

Bauman DE, and W.B. Currie. 1980. Partitioning of nutrients during pregnancy and lactation: a review of mechanisms involving homeostasis and homeorhesis. J. Dairy Sci. 63, 1514-1529.

Bell, A. W. 1995. Regulation of organic nutrient metabolism during transition from late pregnancy to early lactation. J. Anim. Sci. 73:2804-2819.

Bell, A. W., Slepetis, R., and R. A. Ehrhardt. 1995. Growth and accretion of energy and protein in the gravid uterus during late pregnancy. J. Dairy Sci. 78:1954-1961. 
Bell, A. W. and D. E. Bauman. 1997. Adaptations of glucose metabolism during pregnancy and lactation. J Mammary Gland Biol Neoplasia 2:265-278.

Bertoni, G., E. Trevisi, X. Han, and M. Bionaz. 2008. Effects of inflammatory conditions on liver activity in puerperium period and consequences for performance in dairy cows. J. Dairy Sci. 91:3300-310.

Bikman, B.T., S.A. Summers. 2011. Ceramides as modulators of cellular and whole-body metabolism. J. Clin. Invest. 121:4222-4230.

Bleicher, R.J., and M.C. Cabot. 2002. Glucosylceramide synthase and apoptosis. Biochim. Biophys Acta. 1585:172-178.

Bobe, G., J.W. Young, and D.C. Beitz. Invited review: pathology, etiology, prevention, and treatment of fatty liver in dairy cows. 2004. J. Dairy Sci. 87:3105-24.

Boden, G. 1997. Role of fatty acids in the pathogenesis of insulin resistance and NIDDM. Diabetes 46:3-10.

Boden, G., Jadali, F., White, J., Liang, Y., Mozzoli, M., Chen, X., Coleman, E., and C. Smith. . 1991. Effects of fat on insulin-stimulated carbohydrate metabolism in normal men. J. Clin. Invest. 88, 960-966.

Boon, J., A.J. Hoy, R. Stark, R. D. Brown, R. C. Meex, D. C. Henstridge, S. Schenk, P. J. Meikle, J. F. Horowitz, B. A. Kingwell, C. R. Bruce, and M. J. Watt. 2013. Ceramides contained in LDL are elevated in type 2 diabetes and promote inflammation and skeletal muscle insulin resistance. Diabetes 62:401-410.

Boucher J., A. Kleinridders, and C.R. Kahn. 2014. Insulin receptor signaling in normal and insulinresistant states. Cold. Spring. Harb. Perspect. Biol. 6:1.

Boulgaropoulos, B., Amenitsch, H., Laggner, P., and G. Pabst. 2010. Implication of sphingomyelin/ceramide molar ratio on the biological activity of sphingomyelinase. Biophys. J. 99:499-506. 21.

Bourbon, N.A., Sandirasegarane, L., and M. Kester. 2002. Ceramide-induced inhibition of Akt is mediated through protein kinase Czeta: implications for growth arrest. J Biol Chem 277, 3286-3292.

Bradford, B.J., Gour, A.D., Nash, A.S. and M.S. Allen. 2006. Propionate Challenge Tests Have Limited Value for Investigating Bovine Metabolism. J. Nutr. 136, 1915-1920.

Bradford, B.J., Mamedova, L.K., Minton, J.E., Drouillard, J.S., and B.J. Johnson. 2009. Daily injection of tumor necrosis factor- $\{$ alpha $\}$ increases hepatic triglycerides and alters transcript abundance of metabolic genes in lactating dairy cattle. J. Nutr. 139:1451-6

Capper, J.L., R.A. Cady, and D.E. Bauman. 2009. The environmental impact of dairy production: 
1944 compared with 2007. J. Anim. Sci. 87:2160-7.

Chabowski, A., Coort, S.L., Calles-Escandon, J., Tandon, N.N., Glatz, J.F., Luiken, J.J., and A. Bonen. 2004. Insulin stimulates fatty acid transport by regulating expression of FAT/CD36 but not FABPpm. Am. J. Physiol. Endocrinol. Metab. 287:E781-E789.

Chagas, L.M., M.C. Lucy, P.J. Back, D. Blache, J.M. Lee, P.J. Gore, A.J. Sheahan, J.R. Roche. 2009. Insulin resistance in divergent strains of Holstein-Friesian dairy cows offered fresh pasture and increasing amounts of concentrate in early lactation. J Dairy Sci. 2009 92:21622.

Chan, S.J. and D. F. Steiner. 2000. Insulin through the ages: Phylogeny of a growth promoting and metabolic regulatory hormone. Amer. Zool. 40:213-222

Chavez, J. A., and S. A. Summers. 2012. A ceramide-centric view of insulin resistance. Cell Metab. 15:585-594.

Chavez, J. A., M. M. Siddique, S. T. Wang, J. Ching, J. A. Shayman, and S. A. Summers. 2014. Ceramides and glucosylceramides are independent antagonists of insulin signaling. J. Biol. Chem. 289:723-734.

Chavez, J.A., Holland, W.L., Bar, J., Sandhoff, K., and S.A. Summers. 2005. Acid ceramidase overexpression prevents the inhibitory effects of saturated fatty acids on insulin signaling. $\mathbf{J}$ Biol Chem 280:20148-20153

Chavez, J.A., T.A. Knotts, L. P. Wang, G. Li, R.t. Dobrowsky, G.L Florant, S.A. Summers. 2003. A role for ceramide, but not diacylglycerol, in the antagonism of insulin signal transduction by saturated fatty acids. J. Biol. Chem. 278:10297-303.

Checa, A., Holm T., Sjödin, M.O., Reinke, S.N., Alm J., Scheynius, A., and C.E. Wheelock. 2015. Lipid mediator profile in vernix caseosa reflects skin barrier development. Sci Rep. 5: 15740

Chiu, H.C., Kovacs, A., Blanton, R.M., Han, X., Courtois, M., Weinheimer, C.J., Yamada, K.A., Brunet, S., Xu, H., Nerbonne, J.M., Welch, M.J., Fettig, N.M., Sharp, T.L., Sambandam, N., Olson, K.M., Ory, D.S., and J.E. Schaffer. 2005. Transgenic expression of fatty acid transport protein 1 in the heart causes lipotoxic cardiomyopathy. Circ. Res. 96: 225-233.

Christensen, R. A., J. K. Dracklev, D. W. Lacount, and J. H. Clark. 1994. Infusion of four long chain fatty acid mixtures into the abomasum of lactating dairy cows. J. Dairy Sci. 77:1052- 1069.

Claus, R.A., Dorer, M.J., Bunck, A.C., and H.P. Deigner. 2009. Inhibition of sphingomyelin hydrolysis: targeting the lipid mediator ceramide as a key regulator of cellular fate. Curr. Med. Chem. 16:1978-2000 
Contreras, G. A., N. J. O’boyle, T. H. Herdt, and L. M. Sordillo. 2010. Lipomobilization in periparturient dairy cows influences the composition of plasma nonesterified fatty acids and leukocyte phospholipid fatty acids. J. Dairy Sci. 93:2508-2516.

Coppock, C. E., and D. L. Wilks. 1991. Supplemental fat in high- energy rations for lactating cows: Effects on intake, digestion, milk yield and composition. J. Anim. Sci. 69:3826-3837.

Cross, D.A., Alessi, D.R., Cohen, P., Andjelkovich, M., and Hemmings, B.A. 1995. Inhibition of glycogen synthase kinase-3 by insulin mediated by protein kinase B. Nature. 378:785-9.

Cuschieri, J., E. Bulger, J. Billgrin, I. Garcia, and R. V. Maier. 2007. Acid sphingomyelinase is required for lipid Raft TLR4 complex formation. Surg. Infect. (Larchmt) 8:91-106.

Czech, M. P. 1984. The nature and regulation of the insulin receptor. Annu. Rev. Physiol. 47:357381.

Dechow, C.D., G.W. Rogers, L. Klei, T.J. Lawlor, and P.M. VanRaden. 2004. Body condition scores and dairy form evaluations as indicators of days open in US Holsteins. J Dairy Sci. 87:3534-41.

De Koster, J.D., G. Opsomer. 2013. Insulin resistance in dairy cows. Vet. Clin. North. Am. Food. Anim. Pract. 2:299-322.

De Meyts P, Roth J, Neville DM Jr, Gavin JR 3rd, Lesniak MA. 1973. Insulin interactions with its receptors: experimental evidence for negative cooperativity. Biochem. Biophys. Res. Commun. 55:154-161

De Meyts, P., Bianco, A.R., and J. Roth. 1976. Site-site interactions among insulin receptors: characterization of the negative cooperativity. J. Biol. Chem. 251:1877-1888

DeFronzo, R.A. 2004. Dysfunctional fat cells, lipotoxicity and type 2 diabetes. Int. J. Clin. Pract. Suppl. 9-21.

DeFronzo, R.A. 2004. Pathogenesis of type 2 diabetes mellitus. Med. Clin. N. Am. 88:787-835

Dimitriadis, G., Mitrou, P., Lambadiari, V., Maratou, E., and S.A. Raptis. 2011. Insulin effects in muscle and adipose tissue.Diabetes Res Clin Pract. 2011 Aug;93 Suppl 1:S52-9

Doering, T., Brade, H., and K. Sandhoff. Sphingolipid metabolism during epidermal barrier development in mice. J. Lipid Res. 2002. 43:1727-33.

Dokken, B.B., Saengsirisuwan, V., Kim, J.S., Teachey, M.K., and E.J. Henriksen. 2008. Oxidative stress-induced insulin resistance in rat skeletal muscle: Role of glycogen synthase kinase-3. Am. J. Physiol. Endocrinol. Metab. 294: E615- E621.

Drackley, J. K., and J. B. Andersen. 2006. Splanchnic metabolism of long-chain fatty acids in ruminants. Pages 199-224 in Ruminant Physiology: Digestion, Metabolism and Impact of 
Nutrition on Gene Expression, Immunology and Stress. K. Sejrsen, T. Hvelplund, and M. O. Nielsen, ed. Wageningen Academic Publ., Wageningen, the Netherlands.

Drackley, J. K., H. M. Dann, G. N. Douglas, N. A. Janovick Guretzky, N. B. Litherland, J. P. Underwood, and J. J. Loor. 2005. Physiological and pathological adaptations in dairy cows that may increase susceptibility to periparturient diseases and disorders. Ital. J. Anim. Sci. $4: 323-344$.

Dubé, J. J., F. Amati, F. G. S. Toledo, M. Stefanovic-Racic, A. Rossi, P. Coen, and B. H. Goodpaster. 2011. Effects of weight loss and exercise on insulin resistance, and intramyocellular triacylglycerol, diacylglycerol and ceramide. Diabetologia 54:1147-1156.

Duhlmeier, R., Hacker, A., Widdel, A., von Engelhardt, W., and H.P. Sallmann. 2005. Mechanisms of insulin-dependent glucose transport into porcine and bovine skeletal muscle. Am. J. Physiol. Regul. Integr. Comp. Physiol. 289:R187-97.

Ebberink, R.H.M., A.B. Smit, and J.Van Minnen. 1989. The Insulin Family: Evolution of Structure and Function in Vertebrates and Invertebrates. BioLBull.177:176-182.

Edgerton, D.S., Lautz, M., Scott, M., Everett, C.A., Stettler, K.M., Neal, D.W., Chu, C.A., and , A.D. Cherrington. 2006. Insulin's direct effects on the liver dominate the control of hepatic glucose production. J. Clin Invest. 116:521-527.

Edinger, A.L., and C.B. Thompson. 2002. Akt maintains cellsize and survival by increasing mTOR-dependent nutrient uptake. Mol. Biol. Cell. 13:2276-2288.

Eley, R. M., W. W. Thatcher, F. W. Bazer, C. J. Wilcox, R. B. Becker, H. H. Head, and R. W. Adkinson. 1978. Development of the conceptus in the bovine. J. Dairy Sci. 61:467-473

Erion, D.M., and G.I. Shulman. 2010. Diacylglycerol-mediated insulin resistance. Nat. Med. 4:400-2.

Evans, J.L., Maddux, B.A., and I.D. Goldfine, 2005. The molecular basis for oxidative stressinduced insulin resistance. Antioxid. Redox. Signal. 7: 1040-1052.

Ferreira, L.D., Pulawa, L.K., Jensen, D.R., and R.H Eckel. 2001. Overexpressing human lipoprotein lipase in mouse skeletal muscle is associated with insulin resistance. Diabetes 50: 1064-1068

Freychet, P., J. Roth, and D. M. Neville, Jr. 1971. Insulin receptors in the liver: specific binding of (125 I) insulin to the plasma membrane and its relation to insulin bioactivity. Proc. NatL. Acad. Sci. USA. 68:1833-1837

Fridlyand, L.E., and L.H. Philipson. 2006. Reactive species and early manifestation of insulin resistance in type 2 diabetes. Diabetes Obes. Metab. 8: 136-145. 
Fronk, T. J., L. H. Schultz, and A. R. Hardie. 1980. Effect of dry period overconditioning on subsequent metabolic disorders and performance of dairy cows. J. Dairy Sci. 63:1080-1090.

Funaki, M. 2009. Saturated fatty acids and insulin resistance. J. Med. Invest. 56:88-92.

Gault, C.R., L.M. Obeid, Y.A. Hannun. 2010. An overview of sphingolipid metabolism: from synthesis to breakdown. Adv. Exp. Med. Biol. 688:1-23.

Gavin, J.R. III, Roth, J., Neville, D.M. Jr, De Meyts, P., and D.N. Buen. 1974. Insulin-dependent regulation of insulin receptor concentrations: a direct demonstration in cell culture. Proc. Natl. Acad. Sci. USA. 71:84-88

Graber, M., S. Kohler, T. Kaufmann, M. G. Doherr, R. M. Bruckmaier, and H. A. van Dorland. 2010. A field study on characteristics and diversity of gene expression in the liver of dairy cows during the transition period. J. Dairy Sci. 93:5200-5215.

Grummer, R.R., Mashek, D.G., and A. Hayirli A. 2004. Dry matter intake and energy balance in the transition period. Vet. Clin. North Am. Food Anim. Pract. 20:447-70.

Hager, S.R., Jochen, A.L., Kalkhoff, R.K. 1991. Insulin resistance in normal rats infused with glucose for 72 h. Am. J. Physiol. 260: E353-E362.

Hall, M. B. 2009. Determination of starch, including maltooligosaccharides, in animal feeds:

Comparison of methods and a method recommended for aoac collaborative study. J. AOAC Int. 92:42-49.

Hammad, S. M. 2011. Blood sphingolipids in homeostasis and pathobiology. Adv. Exp. Med. Biol. 721, 57-66.

Hannun Y.A., and L.M. Obeid. 2008. Principles of bioactive lipid signalling: lessons from sphingolipids. Nat. Rev. Mol. Cell. Biol. 9:139-150.

Haughey, N. J., R. G. Cutler, A. Tamara, J. C. McArthur, D. L. Vargas, C. A. Pardo, J. Turchan, A. Nath, and M. P. Mattson. 2004. Perturbation of sphingolipid metabolism and ceramide production in HIV-dementia. Ann. Neurol. 55:257-267.

Haughey, N. J., R. G. Cutler, A. Tamara, J. C. McArthur, D. L. Vargas, C. A. Pardo, J. Turchan, A. Nath, and M. P. Mattson. 2004. Perturbation of sphingolipid metabolism and ceramide produciton in HIV-dementia. Ann. Neurol. 55:257-267.

Haus, J. M., S. R. Kashyap, T. Kasumov, R. Zhang, K. R. Kelly, R. A. DeFronzo, and J. P. Kirwan. 2009. Plasma ceramides are elevated in obese subjects with type 2 diabetes and correlate with the severity of insulin resistance. Diabetes 58:337-343.

Haus, J. M., S. R. Kashyap, T. Kasumov, R. Zhang, K. R. Kelly, R. A. DeFronzo, and J. P. Kirwan. 2009. Plasma ceramides are elevated in obese subjects with type 2 diabetes and correlate with the severity of insulin resistance. Diabetes 58:337-343. 
Hammon, H.M., G. Stürmer, F. Schneider, A. Tuchscherer, H. Blum, T. Engelhard, A. Genzel, R. Staufenbiel, and W. Kanitz. 2009. Performance and metabolic and endocrine changes with emphasis on glucose metabolism in high-yielding dairy cows with high and low fat content in liver after calving. J. Dairy Sci. 92:1554-66.

Hedo, J.A., and I.A. Simpson. 1984. Internalization of insulin receptors in the isolated rat adipose cell. Demonstration of the vectorial disposition of receptor subunits. J. Biol. Chem. 259:11083-11089.

Herdt, T.H. 2000. Ruminant adaptation to negative energy balance. Influences on the etiology of ketosis and fatty liver. Vet. Clin. North Am. Food Anim. Pract. 16:215-30.

Hertelendy, F., Machlin, L.J., Takahashi, Y., and Kipnis, D.M. 1968. Insulin release from sheep pancreas in vitro. J. Endocrinol 41, 605-606.

Hirabayashi, Y., Y. Igarashi, and A. H. Merrill Jr. 2006. Sphingolipids synthesis, transport and cellular signaling. Pages 3-22 in Sphingolipid Biology. Y. Hirabayashi, Y. Igarashi, and A. H. Merrill Jr., ed. Springer, Tokyo, Japan.

Holland, W. L., B. T. Bikman, L.-P. Wang, G. Yuguang, K. M. Sargent, S. Bulchand, T. A. Knotts, G. Shui, D. J. Clegg, M. R. Wenk, M. J. Pagliassotti, P. E. Scherer, and S. A. Summers. 2011. Lipid-induced insulin resistance mediated by the proinflammatory receptor TLR4 requires saturated fatty acid-induced ceramide biosynthesis in mice. J. Clin. Invest. 121:1858-1870.

Holland, W. L., J. T. Brozinick, L. P. Wang, E. D. Hawkins, K. M. Sargent, Y. Liu, K. Narra, K. L. Hoehn, T. A. Knotts, A. Siesky, D. H. Nelson, S. K. Karathanasis, G. K. Fontenot, M. J. Birnbaum, and S. A. Summers. 2007. Inhibition of ceramide synthesis ameliorates glucocorticoid-, saturated-fat-, and obesity-induced insulin resistance. Cell Metab 5:167179.

Holland, W. L., J. T. Brozinick, L.-P. Wang, E. D. Hawkins, K. M. Sargent, Y. Liu, K. Narra, K. L. Hoehn, T. A. Knotts, A. Siesky, D. H. Nelson, S. K. Karathanasis, G. K. Fontenot, M. J. Birnbaum, and S. A. Summers. 2007. Inhibition of ceramide synthesis ameliorates glucocorticoid-, saturated-fat-, and obesity-induced insulin resistance. Cell Metab. 5:167179.

Holland, W.L., and S.A. Summers. 2008. Sphingolipids, insulin resistance, and metabolic disease: new insights from in vivo manipulation of sphingolipid metabolism. Endocr. Rev. 4:381-402

Holtenius, K., S. Agenas, C. Delavaud, and Y. Chilliard. 2003. Effects of feeding intensity during the dry period. 2. Metabolic and hormonal responses. J. Dairy Sci. 86:883-891.

Holtenius, P., and K. Holtenius. 2007. A model to estimate insulin sensitivity in dairy cows. Acta Vet. Scand. 49:29.

Homko, C., Sivan, E., Chen, X., Reece, E.A., and G. Boden. 2001. Insulin secretion during and after pregnancy in patients with gestational diabetes mellitus. J. Clin. Endocrinol. Metab. 86: 
568-573.

Horino, M., L. J. Machlin, F. Hertelendy, and D. M. Kipnis. 1968. Effect of short-chain fatty acids on plasma insulin in ruminant and nonruminant species. Endocrinology 83: 118.

Hotamisligil, G.S. 2006. Inflammation and metabolic disorders. Nature. 7121: 860-867

Hotamisligil, G.S. 2010. Endoplasmic reticulum stress and the inflammatory basis of metabolic disease. Cell. 140:900-17.

Hughes, J. P. 1962. A simplified instrument for obtaining liver biopsies in cattle. Am. J. Vet. Res. 23:1111-1113.

Huzzey, J.M., Mann, S., Nydam D.V., Grant, R.J., and T.R. Overton. 2015. Associations of peripartum markers of stress and inflammation with milk yield and reproductive performance in Holstein dairy cows. Prev. Vet. Med. 120(3-:291-297.

Hwang, D., S.H. Rhee. 1999. Receptor-mediated signaling pathways: potential targets of modulation by dietary fatty acids. Am. J. Clin. Nutr. 70, 545-556.

Hyde, R., Peyrollier, K., and H.S. Hundal. 2002. Insulin promotes the cell surface recruitment of the SAT2/ATA2 system A amino acid transporter from an endosomal compartment in skeletal muscle cells. J. Biol. Chem. 277:13628-13634

Ichi, I., K. Nakahara, Y. Miyashita, A. Hidaka, S. Kutsukake, K. Inoue, T. Maruyama, Y. Miwa, M. Harada-Shiba, M. Tsushima, and S. Kojo. 2006. Association of ceramides in human plasma with risk factors of atherosclerosis. Lipids 41:859-863.

Iqbal J, Walsh MT, Hammad SM, Cuchel M, Tarugi P, Hegele RA, Davidson NO, Rader DJ, Klein RL, Hussain MM. 2015. Microsomal Triglyceride Transfer Protein Transfers and Determines Plasma Concentrations of Ceramide and Sphingomyelin but Not Glycosylceramide. J Biol Chem. 290:25863-75

Jaakson, H., K. Ling, J. Samarütel, A. Ilves, T. Kaart, and O. Kärt. 2013. Bood glucose and insulin response of the glucose tolerance test in relation to dairy cow body condition and milk yield. Vet. Med. Zoot. T. 62:28-35.

Janovick-Guretzky, N.A., H. M. Dann, D. B. Carlson, M. R. Murphy, J. J. Loor, and J. K. Drackley. 2007. Housekeeping Gene Expression in Bovine Liver is Affected by Physiological State, Feed Intake, and Dietary Treatment. J. Dairy Sci. 90: 2246-2252.

Jenkins, T. C. 1993. Lipid metabolism in the rumen. J. Dairy Sci. 76:3851-3863

Jheng, H.F., Tsai, P.J., Guo, S.M., Kuo, L.H., Chang, C.S., Su, I.J., Chang, C.R., and Y.S. Tsai. 2012. Mitochondrial fission contributes to mitochondrial dysfunction and insulin resistance in skeletal muscle. Mol. Cell. Biol. 32: 309-319. 
Ji, P., J.S. Osorio, J.K.Drackley, and J.J. Loor. 2012. Overfeeding a moderate energy diet prepartum does not impair bovine subcutaneous adipose tissue insulin signal transduction and induces marked changes in peripartal gene network expression. J Dairy Sci. 95:4333-51.

Kahn, C. R. and M. F. White. 1988. The insulin receptor and the molecular mechanism of insulin action. J. Clin. Invest. 1988. 82: 1151-1156.

Kaske, M., B. Elmahdi, W. von Engelhardt, H.P. Sallmann. 2001. Insulin responsiveness of sheep, ponies, miniature pigs and camels: results of hyperinsulinemic clamps using porcine insulin. J. Comp. Physiol. B. 171:549-556.

Kasuga, M., Karlsson, F.A. and C.R. Kahn. 1982. Insulin stimulates the phosphorylation of the 95,000-dalton subunit of its own receptor. Science 215, 4529:185-187

Kautzky-Willer A, R. Prager, W. Waldhausl, G. Pacini, K. Thomaseth, O.F. Wagner, M. Ulm , C. Streli, and B. Ludvik. 1997. Pronounced insulin resistance and inadequate beta-cell secretion characterize lean gestational diabetes during and after pregnancy. Diabetes Care 20:1717-11233.

Kerestes, M., Faigl, V., Kulcsár, M., Balogh, O., Földi, J., Fébel, H., Chilliard, Y., and G. Huszenicza. 2009. Periparturient insulin secretion and whole-body insulin responsiveness in dairy cows showing various forms of ketone pattern with or without puerperal metritis. Domest Anim Endocrinol. 37:250-61.

Kien, C.L., J.Y. Bunn, M.E. Poynter, R. Stevens, J. Bain, O. Ikayeva, N.K. Fukagawa, C.M. Champagne, K.I. Crain, T.R. Koves, and D.M. Muoio. 2003. Diabetes. 62:1054-63. A lipidomics analysis of the relationship between dietary fatty acid composition and insulin sensitivity in young adults.

Kim, I.H., and G.H., Suh. 2003. Effect of the amount of body condition loss from the dry to near calving periods on the subsequent body condition change, occurrence of postpartum diseases, metabolic parameters and reproductive performance in Holstein dairy cows. Theriogenology. 60:1445-56.

Kim, J.K., Zisman, A., Fillmore, J.J., Peroni, O.D., Kotani, K., Perret, P., Zong ,H., Kahn, C.R., Kahn, B.B., and G.I. Shulman. 2001. Glucose toxicity and the development of diabetes in mice with muscle-specific inactivation of GLUT4. J. Clin. Invest 108: 153-160.

Kitatani, K., Idkowiak-Baldys, J., and Y.A. Hannun. 2008. The sphingolipid salvage pathway in ceramide metabolism and signaling. Cell Signal. 20:1010-1018

Kitatani, K., Sheldon, K., Rajagopalan, V., Anelli, V., Jenkins, R.W., Sun, Y., Grabowski, G.A., Obeid, L.M., and Y.A. Hannun. 2009. Involvement of acid beta-glucosidase 1 in the salvage pathway of ceramide formation. J. Biol. Chem. 284:12972-12978.

Kleppe, B. B., Aiello, R.J., Grummer, R.R., and L.E. Armentano. 1988. Triglyceride accumulation and very low density lipoprotein secretion by rat and goat hepatocytes in vitro.J. Dairy Sci. 
$71: 1813-22$.

Klip, A. 2009. The many ways to regulate glucose transporter 4. Appl. Physiol. Nutr. Metab. $34: 481-487$

Koh, E.H., Lee, W.J., Kim, M.S., Park, J.Y., Lee, I.K., and K.U., Lee. 2005. Intracellular fatty acid metabolism in skeletal muscle and insulin resistance. Curr,.Diabetes Rev. 1: 331-336.

Kolesnick R., and Golde, D.W. 1994. The sphingomyelin pathway in tumor necrosis factor and interleukin-1 signaling. Cell 77, 325-328.

Kolesnick, R. 2002. The therapeutic potential of modulating the ceramide/sphingomyelin pathway. J. Clin. Invest. 110:3-8

Konstantynowicz-Nowicka, K., Harasim, E., Baranowski, M., and A. Chabowski. 2015. New Evidence for the Role of Ceramide in the Development of Hepatic Insulin Resistance. PLoS One. 10: e0116858.

Koonen, D.P., Jacobs, R.L., Febbraio, M., Young, M.E., Soltys, C.L., Ong, H., Vance, D.E., and J.R. Dyck. 2007. Increased hepatic CD36 expression contributes to dyslipidemia associated with diet-induced obesity. Diabetes 56: 2863-2871.

Kowluru, A., and Matti, A. 2012. Hyperactivation of protein phosphatase 2A in models of glucolipotoxicity and diabetes: potential mechanisms and functional consequences. Biochem Pharmacol. 84:591-597.

Kushibiki, S., Hodate, K., Shingu, H., Ueda, Y., Mori. Y., Itoh, T., and Y. Yokomizo. 2001. Effects of long-term administration of recombinant bovine tumor necrosis factor-alpha on glucose metabolism and growth hormone secretion in steers. Am J Vet Res. 62:794-798.

Leahy, J.L., Cooper, H.E., Deal, D.A., Weir, D.G.. 1986. Chronic hyperglycemia is associated with impaired glucose influence on insulin secretion. A study in normal rats using chronic in vivo glucose infusions. J. Clin. Invest. 77: 908-915.

LeBlanc, S. 2010. Monitoring metabolic health of dairy cattle in the transition period. J. Reprod Dev. 56 Suppl:S29-35.

Le Marchand-Brustel, Y., Gual, P., Gremeaux, T., Gonzalez, T., Barrès, R., and J.F. Tanti. 2003. Fatty acid-induced insulin resistance: role of insulin receptor substrate 1 serine phosphorylation in the retroregulation of insulin signalling. Biochem. Soc. Trans 31, 11521156.

Lemmon, M. A. and J. Schlessinger. 2010. Cell signaling by receptor tyrosine kinases. Cell 141, 1117-1134. 
Leroy, J.L., T. Vanholder, A.T. Van Knegsel, I. Garcia-Ispierto, and P.E. Bols. 2008. Nutrient prioritization in dairy cows early postpartum: mismatch between metabolism and fertility? Reprod Domest Anim. 43 Suppl 2:96-103.

Levy, M. and A. H. Futerman. 2010. Mammalian ceramide synthases. IUBMB Life 62:347-356

Lightle, S., R. Tosheva, A. Lee, J. Queen-Baker, B. Boyanovsky, S. Shedlofsky, and M. NikolovaKarakashian. 2003. Elevation of ceramide in serum lipoproteins during acute phase response in humans and mice: Role of serine-palmitoyl transferase. Arch. Biochem. Biophys. 419:120128.

Lipina, C., and H.S. Hundal. 2011. Sphingolipids: Agents provocateurs in the pathogenesis of insulin resistance. Diabetologia. 54:1596-607.

Liu, Y.Y., Hill, R.A., and Y.T., Li. 2013. Ceramide glycosylation catalyzed by glucosylceramide synthase and cancer drug resistance. Adv. Cancer Res. 117:59-89.

Lock, A. L., C. L. Preseault, J. E. Rico, K. E. Deland, and M. S. Allen. 2013. Feeding a c16:0enriched fat supplement increased the yield of milk fat and improved conversion of feed to milk. J. Dairy Sci. 96:6650-6659.

Maia, M. R. G., L. C. Chaudhary, C. S. Bestwick, A. J. Richardson, N. McKain, T. R. Larson, I. A. Graham, and R. J. Wallace. 2010. Toxicity of unsaturated fatty acids to the biohydrogenating ruminal bacterium, Butyrivibrio fibrisolvens. BMC Microbiology $10: 52$

Mann, S., F. A. Leal Yepes, M. Duplessis, J. J. Wakshlag, T. R. Overton, B. P. Cummings, and D. V. Nydam. 2016. Dry period plane of energy: Effects on glucose tolerance in transition dairy cows. J. Dairy Sci. 99:701-717

Manns, J.G. and J.M. Boda. 1967. Insulin release by acetate, propionate, butyrate, and glucose in lambs and adult sheep. Am. J. Phys. 212: 747-755

Mathews, A. T., J. E. Rico, N. T. Sprenkle, A. L. Lock, and J. W. McFadden. 2016. Increasing palmitic acid intake enhances milk production and prevents glucose-stimulated NEFA disappearance without modifying systemic glucose tolerance in mid-lactation dairy cows. J. Dairy Sci. Submitted.

McCann, J.P. and T.J. Reimers. 1985. Insulin response to glucose in estrous and diestrous obese and lean heifers. J Anim Sci. 61:619-23.

McNamara, J. P. 1991. Regulation of adipose tissue metabolism in support of lactation. J. Dairy Sci. 74:706-719.

McNamara, J. P. and J. K. Hillers. 1986. Adaptations in lipid metabolism of bovine adipose tissue in lactogenesis and lactation J. Lipid Res. 27:150-157. 
Mei, J., L. S. Holst, T. R. Landstrom, C. Holm, D. Brindley, V. Manganiello, and E. Degerman. 2002. C2-ceramide influences the expression and insulin-mediated regulation of cyclic nucleotide phosphodiesterase 3B and lipolysis in 3T3-L1 adipocytes. Diabetes 51:631637.

Meikle, P. J., G. Wong, C. K. Barlow, J. M. Weir, M. A. Greeve, G. L. MacIntosh, L. Almasy, A. G. Comuzzie, M. C. Mahaney, A. Kowalczyk, I. Haviv, N. Grantham, D. J. Magliano, J. B. M. Jowett, P. Zimmet, J. E. Curran, J. Blangero, and J. Shaw. 2013. Plasma lipid profiling shows similar associations with prediabetes and type 2 diabetes. PLoS ONE 8:e74341.

Menting, J.G., J. Whittaker, M.B. Margetts, L.J. Whittaker, G.K. Kong, B.J. Smith, C.J. Watson, L. Záková, E. Kletvíková, J. Jiráček, S.J. Chan, D.F. Steiner, G.G. Dodson, A.M. Brzozowski, M.A. Weiss, C.W. Ward, and M.C. Lawrence. 2013. How insulin engages its primary binding site on the insulin receptor. Nature. 493:241-5.

Merrill A.H. Jr., M. D. Wang, M. Park, and M. C. Sullards. 2007. (Glyco)sphingolipidology: an amazing challenge and opportunity for systems biology. Trends in Biochemical Sciences. 32, 10: 457-468.

Merrill, A. H. Jr., T. H. Stokes, A. Momin, H. Park, B. J. Portz, S. Kelly, E. Wang, M. C. Sullards, and M. D. Wang. 2009. Sphingolipidomics: A valuable tool for understanding the roles of phingolipids in biology and disease. J. Lipid Res. 50 Suppl:S97-102.

Merrill, A.H. Jr., 2002. De novo sphingolipid biosynthesis: a necessary, but dangerous, pathway. J. Biol. Chem. 277:25843-25846.

Merrill, A.H. Jr., Sullards, M.C., Allegood, J.C., Kelly, S., and E. Wang. 2005. Sphingolipidomics: high-throughput, structure-specific, and quantitative analysis of sphingolipids by liquid chromatography tandem mass spectrometry. Methods 36, 207-224.

Mihalik, S.J., B.H. Goodpaster, D.E. Kelley, D.H. Chace, J. Vockley, F.G. Toledo, and J.P. DeLany. 2010. Increased levels of plasma acylcarnitines in obesity and type 2 diabetes and identification of a marker of glucolipotoxicity. Obesity (Silver Spring). 18:1695-1700.

Mills, J.A.N., France, J., and J. Dijkstra. 1999. A review of starch digestion in the lactating dairy cow and proposals for a mechanistic model: (1) Dietary starch characterisation and ruminal starch digestion. J. Anim. Feed Sci. 8:291-340.

Morrow DA. 1976. Fat cow syndrome. J. Dairy Sci. 59:1625-1629.

Muggeo, M., B. H. Ginsberg, J. Roth, D. M. Neville, Jr., P. DeMeyts, and C. R. Kahn. 1979. The insulin receptor in vertebrates is functionally more conserved during evolution than insulin itself. Endocrinology. 104:1383-1392.

Myers, M.G. Jr, Backer, J.M., Sun, X.J., Shoelson, S., Hu, P., Schlessinger, J., Yoakim, M., Schaffhausen, B., and M.F. White. 1992. IRS-1 activates phosphatidylinositol 30 -kinase by associating with src homology 2 domains of p85. Proc. Natl. Acad. Sci. 89: 10350-10354 
Nguyen, M.T., Favelyukis, S., Nguyen, A.K., Reichart, D., Scott, P.A., Jenn, A., Liu-Bryan, R., Glass, C.K., Neels, J.G., J.M. Olefsky. 2007. A subpopulation of macrophages infiltrates hypertrophic adipose tissue and is activated by free fatty acids via Toll-like receptors 2 and 4 and JNK-dependent pathways. J. Biol. Chem. 282, 35279-35292.

Nguyen, M.T., Satoh, H., Favelyukis, S., Babendure, J.L., Imamura, T., Sbodio, J.I., Zalevsky, J., Dahiyat, B.I., Chi, N.W., and J.M. Olefsky. 2005. JNK and tumor necrosis factor-alpha mediate free fatty acid-induced insulin resistance in 3T3-L1 adipocytes. J. Biol. Chem. 280, 35361-35371.

NRC. 2001. Nutritional Requirements of Dairy Cattle. 7th rev. ed. Natl. Acad. Sci., Washington, DC.

O'Boyle, N, C.M. Corl, J.C. Gandy, and L.M. Sordillo. 2006. Relationship of body condition score and oxidant stress to tumor necrosis factor expression in dairy cattle. Vet. Immunol. Immunopathol. 113:297-304.

Ohtsuka, H, M. Koiwa, A. Hatsugaya, K. Kudo, F. Hoshi, N. Itoh, H. Yokota, H. Okada, and S. Kawamura. 2001. Relationship between serum TNF activity and insulin resistance in dairy cows affected with naturally occurring fatty liver. J. Vet. Med. Sci. 63, 1021-1025.

Oikawa, S., and Oetzel GR. 2006. Decreased insulin response in dairy cows following a four-day fast to induce hepatic lipidosis. J Dairy Sci. 89:2999-3005.

Osborn, O., and J.M. Olefsky JM. 2012. The cellular and signaling networks linking the immune system and metabolism in disease. Nat. Med. 18: 363-374.

Ospina, P.A., Nydam, D.V., Stokol, T., and T.R. Overton. 2010a. Associations of elevated nonesterified fatty acids and beta-hydroxybutyrate concentrations with early lactation reproductive performance and milk production in transition dairy cattle in the northeastern United States. J. Dairy Sci. 93:1596-1603.

Ospina, P.A., Nydam, D.V., Stokol, T., and T.R. Overton. 2010b. Evaluation of nonesterified fatty acids and beta-hydroxybutyrate in transition dairy cattle in the northeastern United States: Critical thresholds for prediction of clinical diseases.J Dairy Sci. 93:546-554.

Ozcan, U., Cao, Q., Yilmaz, E., Lee, A.H., Iwakoshi, N.N., Ozdelen, E., Tuncman, G., Gorgun, C., Glimcher, L.H., Hotamisligil, G.S. 2004. Endoplasmic reticulum stress links obesity, insulin action, and type 2 diabetes. Science 306: 457-461

Pal, A., Barber, T.M., Van de Bunt, M., Rudge, S.A., Zhang, Q., Lachlan, K.L., Cooper, N.S., Linden, H., Levy, J.C., Wakelam, M.J., et al. 2012. PTEN mutations as a cause of constitutive insulin sensitivity and obesity. N. Engl. J. Med. 367, 1002-1011

Palmquist, D.L., Jenkins, T.C., 1980. Fat in lactation rations: review. J. Dairy Sci. 63:1-14. 
Park, J.-W., and Y. Pewzner-Jung. 2013. Ceramide synthases: Reexamining longevity. Pages 89107 in Sphingolipids: Basic Science and Drug Development. Vol. 215. E. Gulbins and I. Petrache, ed. Springer, Vienna, Italy.

Paz, K., Hemi, R., LeRoith, D., Karasik, A., Elhanany, E., Kanety, H., and Y. Zick. 1997. A molecular basis for insulin resistance: elevated serine/threonine phosphorylation of IRS-1 and IRS-2 inhibits their binding to the juxtamembrane region of the insulin receptor and impairs their ability to undergo insulin-induced tyrosine phosphorylation. J. Biol. Chem. 272:2991129918

Peraldi, P., Hotamisligil, G.S., Buurman, W.A, White, M.F., and Spiegelman, B.M.. 1996. Tumor necrosis factor (TNF)-alpha inhibits insulin signaling through stimulation of the p55 TNF receptor and activation of sphingomyelinase. J. Biol. Chem. 271:13018-13022.

Petersen, K.F., and G.I. Shulman. 2006. Etiology of insulin resistance. Am. J. Med. 119: S10-S16,

Piantoni, P., A. L. Lock, and M. S. Allen. 2013. Palmitic acid increased yields of milk and milk fat and nutrient digestibility across production level of lactating cows. J. Dairy Sci. 96:7143-7154.

Pires, J. A., A. H. Souza, and R. R. Grummer. 2007a. Induction of hyperlipidemia by intravenous infusion of tallow emulsion causes insulin resistance in Holstein cows. J. Dairy Sci. 90:27352744.

Pires, J. A., J. B. Pescara, and R. R. Grummer. 2007b. Reduction of plasma NEFA concentration by nicotinic acid enhances the response to insulin in feed-restricted Holstein cows. J. Dairy Sci. 90:4635-4642.

Pullen, D. L., D. L. Palmquist, and R. S. Emery. 1989. Effect of days of lactation and methionine hydroxy analog on incorporation of plasma fatty acids into plasma triglycerides. J. Dairy Sci. $72: 49$.

Reaven, G. M., C. Hollenbeck, C.-Y. Jeng, M. S. Wu, and Y.-D. I. Chen. 1988. Measurement of plasma glucose, free fatty acid, lactate, and insulin for $24 \mathrm{~h}$ in patients with NIDDM. Diabetes 37:1020-1024.

Reaven, G.M. 1988. Banting lecture 1988. Role of insulin resistance in human disease. Diabetes. 37:1595-607.

Reynolds, C. K., P. C. Aikman, B. Lupoli, D. J. Humphries, and D. E. Beever. 2003. Splanchnic metabolism of dairy cows during the transition from late gestation through early lactation. J. Dairy Sci.86:1201-1217.

Relling, A. E., and C. K. Reynolds. 2007. Feeding rumen-inert fats differing in degree of saturation decreases intake and increases plasma concentrations of gut peptides in lactating dairy cows. J. Dairy Sci. 90:1506-1515. 
Riboni, L., Bassi, R., Caminiti, A., Prinetti, A., Viani, P., and G. Tettamanti. 1998. Metabolic fate of exogenous sphingosine in neuroblastoma neuro2A cells. Dosedependence and biological effects. Ann. N. Y. Acad. Sci. 845:46-56

Rico, D. E., Y. Ying, and K. J. Harvatine. 2014b. Effect of a high-palmitic acid fat supplement on milk production and apparent total-tract digestibility in high- and low-milk yield dairy cows. J. Dairy Sci. 97:3739-3751.

Rico, J. E., M. S. Allen, and A. L. Lock. 2014a. Compared with stearic acid, palmitic acid increased the yield of milk fat and improved feed efficiency across production level of cows. J. Dairy Sci. 97:1057-1066.

Rico, J. E., V. V. R. Bandaru, J. M. Dorskind, N. J. Haughey, and J. W. McFadden. 2015. Plasma ceramides are elevated in overweight Holstein dairy cows experiencing greater lipolysis and insulin resistance during the transition from late pregnancy to early lactation. J Dairy Sci. 98:7757-70.

Robinson, K.A., and M.G. Buse. 2008. Mechanisms of high-glucose/insulin-mediated desensitization of acute insulin-stimulated glucose transport and Akt activation. Am. J. Physiol. Endocrinol. Metab. 29:E870-81.

Roche, J.R., J.M. Lee, K.A. Macdonald, and D.P. Berry. 2007. Relationships among body condition score, body weight, and milk production variables in pasture-based dairy cows. J. Dairy Sci. 90:3802-3815.

Roche, J.R., N.C. Friggens, J.K.Kay, M.W. Fisher, K.J. Stafford, and D.P. Berry. 2009. Invited review: Body condition score and its association with dairy cow productivity, health, and welfare. J. Dairy Sci. 92: 5769-5801.

Roden, M., Price, T.B., Perseghin, G., Petersen, K.F., Rothman, D.L. Cline, G.W., and G.I. Shulman. 1996. Mechanism of free fatty acid-induced insulin resistance in humans. J. Clin Invest. 97, 2859-2865.

Rudich, A., Tirosh, A., Potashnik, R., Hemi, R., Kanety, H., and N. Bashan. 1998. Prolonged oxidative stress impairs insulin-induced GLUT4 translocation in 3T3-L1 adipocytes. Diabetes 47: 1562-1569.

Rukkwamsuk, T., T. A. M. Kruip, and T. Wensing. 1999. Relationship between overfeeding and overconditioning in the dry period and the problems of high-producing dairy cows during the postparturient period. Vet. Q. 21:71-77.

Ryan, E. A., M. J. O’Sullivan, and J. S. Skyler. 1985. Insulin action during pregnancy. Studies with the euglycemic clamp technique. Diabetes 34:380-389

Sadri, H., Bruckmaier, R. M., Rahmani, H. R., Ghorbani, G. R., Morel, I., and H. A. Van Dorland. Gene expression of tumour necrosis factor and insulin signaling-related factors in 
subcutaneous adipose tissue during the dry period and in early lactation in dairy cows. J. Anim. Physiol. Anim. Nutr., 94: e194-e202.

Saltiel, A.R., and C.R. Kahn. 2001. Insulin signalling and the regulation of glucose and lipid metabolism. Nature 414:799-806, 2001

Samad, F., Hester, K.D., Yang, G., Hannun, Y.A., and J. Bielawski. 2006. Altered adipose and plasma sphingolipid metabolism in obesity: a potential mechanism for cardiovascular and metabolic risk. Diabetes 55:2579-2587

Sanger, F., and H. Tuppy. 1951. The amino-acid sequence in the phenylalanyl chain of insulin. I. The identification of lower peptides from partial hydrolysates. Biochem. J. 49: 463-81

Sano H, Arai H, Takahashi, A., Takahashi, H., and Y. Terashima. 1999. Insulin and glucagon responses to intravenous injections of glucose, arginine and propionate in lactating cows and growing calves. Can. J. Anim. Sci.79, 309-314.

Sarbassov, D.D., Guertin, D.A., Ali, S.M., and D.M. Sabatini. 2005. Phosphorylation and regulation of Akt/PKB by the rictor-mTOR complex. Science 307: 1098-1101

Sasaki, S. 2002. Mechanism of insulin action on glucose metabolism in ruminants. Anim. Sci. 73, 6: $423-433$

Schenk, S., M. Saberi, and J.M. Olefsky. 2008. Insulin sensitivity: modulation by nutrients and inflammation. J. Clin Invest. 118:2992-3002.

Schoenberg, K. M., R. M. Ehrhardt, and T. R. Overton. 2012. Effects of plane of nutrition and feed deprivation on insulin responses in dairy cattle during late gestation. J Dairy Sci 95:670-682.

Senn, J.J. 2006. Toll-like receptor-2 is essential for the development of palmitate-induced insulin resistance in myotubes. J. Biol. Chem. 281:26865-26875.

Sevillano, J., J. de Castro, C. Bocos, E. Herrera, and M. P. Ramos. 2007. Role of insulin receptor substrate-1 serine 307 phosphorylation and adiponectin in adipose tissue insulin resistance in late pregnancy. Endocrinology 148:5933-5942.

Shanik, M.H., Xu, Y., Skrha, J., Dankner, R., Zick, Y., and J. Roth. 2008 .Insulin resistance and hyperinsulinemia: is hyperinsulinemia the cart or the horse?. Diabetes Care. Suppl 2:S262-8.

Shaw, L.M. 2011. The insulin receptor substrate (IRS) proteins: At the intersection of metabolism and cancer. Cell Cycle 10: 1750-1756.

Shi, H., Kokoeva, M.V., Inouye, K., Tzameli, I., Yin, H., and J.S. Flier. 2006. TLR4 links innate immunity and fatty acid-induced insulin resistance. J. Clin. Invest. 116, 3015-3025.

Shimabukuro, M., Zhou, Y.T., Levi, M, and R.H. Unger. 1998. Fatty acid-induced beta cell 
apoptosis: a link between obesity and diabetes. Proc. Natl. Acad. Sci. USA 95, 2498-2502.

Signorelli, P., Avagliano, L., Reforgiato, M.R., Toppi, N., Casas, J., Fabriàs, G., Marconi, A.M., Ghidoni, R., and A. Caretti. 2016. De novo ceramide synthesis is involved in acute inflammation during labor. Biol. Chem. 397:147-55.

Sjoholm, A. 1995. Ceramide inhibits pancreatic beta-cell insulin production and mitogenesis and mimics the actions of interleukin-1 beta. FEBS Lett. 367, 283-286.

Sordillo, L.M, G.A. Contreras, and S.L. Aitken. 2009. Metabolic factors affecting the inflammatory response of periparturient dairy cows. Anim Health Res Rev. 10:53-63.

Sordillo, L.M., and W. Raphael. 2013. Significance of metabolic stress, lipid mobilization, and inflammation on transition cow disorders. Vet. Clin. North Am. Food Anim. Pract. 29:267-78.

Spachmann, S. K. , U. Schönhusen, B. Kuhla, M. Röntgen, and H. M. Hammon. 2013. Insulin signaling of glucose uptake in skeletal muscle of lactating dairy cows. Pages 277-278 in Energy and Protein Metabolism and Nutrition in Sustainable Animal Production: 4th International Symposium on Energy and Protein Metabolism and Nutrition, Sacramento, California, USA, 9-12 September 2013. Vol. 134. J. W. Oltjen, E. Kebreab, and Hélène Lapierre, ed. Wageningen Academic Pub. The Netherlands.

Starke, A., A. Haudum, R. Busche, M. Beyerbach, S. Danicke, and J. Rehage. 2010. Technical note: Analysis of total lipid and triacylglycerol content in small liver biopsy samples in cattle. J. Anim. Sci. 88:2741-2750.

Steiner, D. F., S. J. Chan, J. M. Welsh, and S. C. M. Kwok. 1985. Structure and evolution of the insulin gene. Annu. Rev. Genet. 19:463-84.

Straczkowski, M., Kowalska, I., Baranowski, M., Nikolajuk, A., Otziomek, E., Zabielski, P., Adamska, A., Blachnio, A., Gorski, J., and M. Gorska. 2007. Increased skeletal muscle ceramide level in men at risk of developing type 2 diabetes. Diabetologia 50, 2366-2373

Stratford, S., Hoehn, K.L., Liu, F., and S.A. Summers. 2004. Regulation of insulin action by ceramide: dual mechanisms linking ceramide accumulation to the inhibition of Akt/protein kinase B. J. Biol. Chem. 279, 36608-36615.

Summers, S. A., Garza LA, Zhou H, and Birnbaum MJ. 1998. Regulation of insulin-stimulated glucose transporter GLUT4 translocation and Akt kinase activity by ceramide. Molecular and cellular biology 18: 5457-5464.

Summers, S.A. 2006. Ceramides in insulin resistance and lipotoxicity. Prog. Lipid. Res. 45, 4272.

Sun, X.J., Crimmins D.L., Myers, M.R. Jr, Miralpeix, M., and M.F. White. 1993. Pleiotropic insulin signals are engaged by multisite phosphorylation of IRS-1. Mol. Cell. Biol. 13: 7418- 
7428.

Sun, X.J., Rothenberg, P.L., Kahn, C.R, Backer, J.M., Araki, E., Wilden, P.A., Cahill, D.A., Goldstein, B.J., and M.F. White. 1991. Structure of the insulin receptor substrate IRS-1 defines a unique signal transduction protein. Nature 352: 73-77.

Sundrum, A. 2015. Metabolic Disorders in the Transition Period Indicate that the Dairy Cows' Ability to Adapt is Overstressed. Animals (Basel). 5: 978-1020

Teruel, T., Hernandez, R., and M. Lorenzo. 2001. Ceramide mediates insulin resistance by tumor necrosis factor-alpha in brown adipocytes by maintaining Akt in an inactive dephosphorylated state. Diabetes 50, 2563-2571.

Thévenod, F. 2008. Pathophysiology of diabetes mellitus type 2: Roles of obesity, insulin resistance and $\beta$-Cell dysfunction. Front Diabetes. Pages 1-18 in Diabetes and Cancer: Epidemiological Evidence and Molecular Links. Vol. 19. K. Masur, F. Thévenod, and K. S.Zänker, ed. Karger, Basel, Switzerland.

Tucker, H. A. 1985. Endocrine and neural control of the mammary gland. In: B. L. Larson (Ed.) Lactation. p 39. Iowa State University Press, Ames

Turner, N. 2013. Mitochondrial Metabolism and Insulin Action, Type 2 Diabetes, Prof. Kazuko Masuo (Ed.), InTech, DOI: 10.5772/56449.

Ullrich, A., J. R. Bell, E. Y. Chen, R. Herrera, L. M. Petruzzelli, T. J. Dull, A. Gray, L. Coussens, Y.-C. Liao, M. Tsubokawa, A. Mason, P. H. Seeburg, C. Grunfeld, 0. M. Rosen, and J. Ramachandran. 1985. Human insulin receptor and its relationship to the tyrosine kinase family of oncogenes. Nature. 313:756-761.

Unger, R.H. 2003. Minireview: weapons of lean body mass destruction: the role of ectopic lipids in the metabolic syndrome. Endocrinology 144, 5159-5165.

Ussher, J. R., T. R. Koves, V. J. J. Cadete, L. Zhang, J. S. Jaswal, S. J. Swyrd, D. G. Lopaschuk, S. D. Proctor, W. Keung, and D. M. Muoio. 2010. Inhibition of de novo ceramide synthesis reverses diet-induced insulin resistance and enhances whole-body oxygen consumption. Diabetes 59:2453-2464.

Van Soest, P. J., J. B. Robertson, and B. A. Lewis. 1991. Methods for dietary fiber, neutral detergent fiber, and nonstarch polysaccharides in relation to animal nutrition. J. Dairy Sci. 74:3583-3597.

Varman, T. S., K. F. Petersen, and G. I. Shulman. 2010. Lipid-induced insulin resistance: unravelling the mechanism. Lancet. 375: 2267-2277.

Ward, C.W., and M.C. Lawrence. 2009. Ligand-induced activation of the insulin receptor: a multistep process involving structural changes in both the ligand and the receptor. Bioessays. 4:422-34. 
Watt, M. J., A. C. Barnett, C. R. Bruce, S. Schenk, J. F. Horowitz, and A. J. Hoy. 2012. Regulation of plasma ceramide levels with fatty acid oversupply: Evidence that the liver detects and secretes de novo synthesised ceramide. Diabetologia 55:2741-2746.

Wellen, K.E., and G.S. Hotamisligil. 2005. Inflammation, stress, and diabetes. J. Clin. Invest. 115:1111-1119.

White, M.F. 2006. Regulating insulin signaling and b-cell function through IRS proteins. Can. J. Physiol. Pharmacol. 84: 725-737.

Wildman, E. E., G. M. Jones, P. E. Wagner, and R. L. Bowman. 1982. A dairy cow body condition scoring system and its relationship to selected production characteristics. J. Dairy Sci. 65:495501. Xia, J., R. Mandal, I. Sinelnikov, D. Broadhurst, and D. S. Wishart. 2012. MetaboAnalyst 2.0-A comprehensive server for metabolomic data analysis. Nucleic Acids Res. 40:W127W133.

Xia, J., R. Mandal, Sinelnikov, I., D. Broadhurst, and D.S. Wishart. 2012. MetaboAnalyst 2.0 - a comprehensive server for metabolomic data analysis. Nucl. Acids Res. 40, W127-W133.

Xia, J., I. Sinelnikov, B. Han, and D.S. Wishart. 2015. MetaboAnalyst 3.0 - making metabolomics more meaningful. Nucl. Acids Res. DOI: 10.1093/nar/gkv380.

Yang, Z., Costanzo, M., Golde, D.W., and Kolesnick, R.N. 1993. Tumor necrosis factor activation of the sphingomyelin pathway signals nuclear factor kappa B translocation in intact HL-60 cells. J. Biol. Chem. 268:20520-20523.

Yu, C., Y. Chen, G.W. Cline, D. Zhang, H. Zong, Y. Wang, R. Bergeron, J.K. Kim, S.W. Cushman, G.J. Cooney, B. Atcheson, , M.F. White, E.W. Kraegen, and G.I. Shulman. 2002. Mechanism by Which Fatty Acids Inhibit Insulin Activation of Insulin Receptor Substrate-1 (IRS-1)-associated Phosphatidylinositol 3-Kinase Activity in Muscle. Journal of Biological Chemistry 277, 50230-50236.

Zachut, M., H. Honig, S. Striem, Y. Zick, S. Boura-Halfon, and U. Moallem. 2013. Periparturient dairy cows do not exhibit hepatic insulin resistance, yet adipose-specific insulin resistance occurs in cows prone to high weight loss. J. Dairy Sci. 96:5656-5669.

Zhang, D., Liu, Z.X., Choi, C.S., Tian, L., Kibbey, R., Dong, J., Cline, G.W., Wood, P.A., and G.I. Shulman. 2007. Mitochondrial dysfunction due to long-chain Acyl-CoA dehydrogenase de- ficiency causes hepatic steatosis and hepatic insulin resistance. Proc. Natl. Acad. Sci. 104: $17075-17080$

Zhang, X., Zhang, G., Zhang, H., Karin, M., Bai, H., and D. Cai. 2008. Hypothalamic IKK-b/NF$\mathrm{kB}$ and ER stress link overnutrition to energy imbalance and obesity. Cell 135: 61-73

Zick, Y., Grunberger, G., Podskalny, J.M., Moncada, V., Taylor, S.I., Gorden, P., and J. Roth. 1983. Insulin stimulates phosphorylation of serine residues in soluble insulin receptors. Biochem. Biophys. Res. Commun. 116:1129-1135 